Linköping studies in science and technology. Thesis.

No. 1681

Licentiate's Thesis

\title{
Graybox Modelling of Ships Using Indirect Input Measurements
}

\author{
Jonas Linder
}

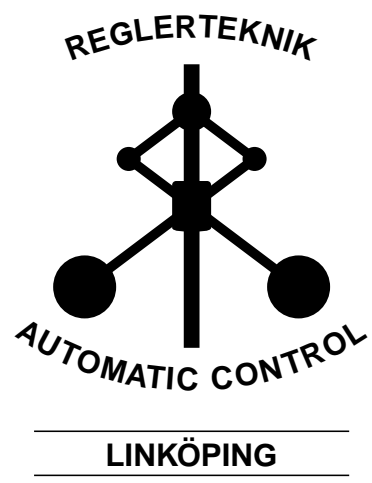

Division of Automatic Control

Department of Electrical Engineering

Linköping University, SE-581 83 Linköping, Sweden

http://www. control.isy.liu.se

jonas.linder@liu.se

Linköping 2014 
This is a Swedish Licentiate's Thesis.

Swedish postgraduate education leads to a Doctor's degree and/or a Licentiate's degree.

A Doctor's Degree comprises 240 ECTS credits (4 years of full-time studies).

A Licentiate's degree comprises 120 ECTS credits,

of which at least 60 ECTS credits constitute a Licentiate's thesis.

Linköping studies in science and technology. Thesis.

No. 1681

\title{
Graybox Modelling of Ships Using Indirect Input Measurements
}

\author{
Jonas Linder \\ jonas.linder@liu.se \\ ww. control.isy.liu.se \\ Department of Electrical Engineering \\ Linköping University \\ SE-581 83 Linköping \\ Sweden
}

ISBN 978-91-7519-222-2

ISSN 0280-7971

Copyright (C) 2014 Jonas Linder

Printed by LiU-Tryck, Linköping, Sweden 2014 
To confusion... 



\section{Abstract}

A ship's roll dynamics is very sensitive to changes in the loading conditions and a worst-case scenario is that the ship will capsize. Actually, the mass and center of mass are two of the most influential parameters in most mechanical systems. However, it is difficult to uniquely estimate these parameters for a ship under normal operational conditions without special experiments or equipment.

Instead of focusing on a sensor-rich environment where all possible signals on a ship can be measured and a complete model of the ship can be estimated, this thesis presents an approach where a model of a subsystem of the ship's dynamics is estimated using only a limited set of sensors. More specifically, the roll dynamics is studied and it is assumed that only motion measurements from an inertial measurement unit (IMU) together with measurements of the rudder angle are available. Hence, direct measurements of the true inputs to the subsystem are not available, but the measurements indirectly contain information about the inputs and these indirect input measurements can be used as a substitute.

To understand the properties of the proposed method, it is applied to an approximate model of the ship's roll dynamics. The analyses show that only a subset of the unknown parameters can be estimated simultaneously and that the estimation problem is similar to closed-loop system identification.

A multi-stage method that uses several datasets is introduced to circumvent the restrictions shown in the identifiability analysis. An iterative closed-loop instrumental variable approach is used to estimate subsets of the parameters in each step. The approach is verified on experimental data with good results.

It is shown that a well-established and more complete ship model can be used to derive a generalization of the approximate model, with more input measurements and a few extra parameters. The generalized model has the same basic properties as the approximate model. The added complexity is due to the ship's interaction with water. Because of this extra complexity, an iterative joint closed-loop instrumental variable approach based on a graybox formulation and using multiple datasets simultaneously is introduced to estimate the parameters.

Finally, experiments with a scale ship model are described. The joint identification method is applied to the collected data and gives promising results. 



\section{Populärvetenskaplig sammanfattning}

Ett skepps beteende är väldigt känsligt för hur det är lastat och om det är fellastat kan det i värsta fall kapsejsa. Skeppets lastförhållande kan beskrivas med hjälp av dess massa och tyngdpunkt, vilka är två av de fysikaliska storheter som har störst inverkan på många mekaniska systems beteende. Tyvärr är det under normala förhållanden svårt att bestämma dessa storheter entydigt för ett skepp utan att göra speciella experiment eller att använda speciell utrustning.

Istället för att fokusera på fall där man kan mäta alla signaler som behövs och skatta en komplett dynamisk modell från dessa så presenteras här ett tillvägagångssätt där endast en del av skeppets totala dynamik modelleras. Dessutom används bara en begränsad uppsättning sensorer. Mer specifikt så är det skeppets rolldynamik som undersöks och det antas att endast mätningar av skeppets rörelser, från tröghetssensorer, och rodervinkeln finns tillgängliga. Detta betyder att direkta mätningar av de sanna insignalerna till rolldynamikdelsystemet inte finns tillgängliga. Å andra sidan innehåller de tillgängliga mätningarna indirekt information om dessa insignaler och mätningarna kan därför användas som ersättning för insignalerna.

För att förstå egenskaperna hos den föreslagna metoden så appliceras den på en förenklad modell av skeppets rolldynamik. Analyserna visar att endast en delmängd av de okända parametrarna i den förslagna modellen kan bestämmas entydigt och att parameterskattningsproblemet liknar systemidentifiering för återkopplade system.

En flerstegsmetod, som använder flera dataset, introduceras för att kringgå vissa av de restriktioner som analysen påvisat. En iterativ parameterskattningsmetod för återkopplade system baserad på korrelation med instrumentvariabler används i varje steg för att skatta de okända parametrarna. Den föreslagna metoden har testas på verkliga experimentdata med goda resultat.

Det visas också att en generalisering av den förenklade modellen kan härledas från en väletablerad och mer komplett skeppsmodell. Den generaliserade modellen har ett fåtal extra parametrar och extra insignaler som resultat av skeppets växelverkan med vattnet. På grund av den ökade komplexiteten införs en modell med fysikaliska parametrar och flera datamängder används samtidigt i en iterativ parameterskattningsmetod för återkopplade system baserad på korrelation med instrumentvariabler.

Slutligen beskrivs ett experiment där data samlats in från en modellskepp i en bassäng. Den gemensamma parameterskattningsmetoden har tillämpats på den insamlade datan och resultaten är lovande. 



\section{Acknowledgements}

I recently had a long discussion with one of my colleagues about the purpose of acknowledgements and the colleague (who surprise surprise, wishes to stay anonymous) said

\section{"Why should you thank anyone?"}

Needless to say, I, for one, think that there are plenty of reasons to thank people and I intend to do it! Every journey has a beginning and an end, mine started in 1984 on the best coast of Sweden in the sunny city of Göteborg... but I will not bore you with all the details. However, let me make a few worthwhile stops along the way! I would like to start by extending my gratitude to my friend Simon Lindkvist. If it were not for you, I would never have done my summer internship at ABB, which led to the Master's thesis that led to where I am right now, thank you! I would also like to thank my former colleagues at ABB Corporate Research for nurturing me towards academia and especially Shiva Sander Tavallaey for saying

"I am giving you this job right now, allowing you time to understand that you want to do a PhD"

when I was hesitant and then, together with Johan Sjöberg, recommended me for a position at Linköping University!

Talking about the university, I hope that everyone in our group understands what a wonderful place we are working at and I would like to extend my gratitude to Svante Gunnarsson for always having an open door, for the opportunity to make my mark in the history books and his partner in crime, Ninna Stensgård, for excellent help with all kinds of administrative issues. Without guidance my sense of direction would be lost and I would like to acknowledge my co-supervisors Fredrik Gustafsson and Johan Sjöberg for good ideas, discussions and a fruitful collaborations. I especially would like to thank my supervisor Martin Enqvist for having enough patience and the energy to guide me! Your eye for detail and care put into every single thing are nothing but amazing! You are a constant source of inspiration, however, I wonder who is most happy about the thesis being finished, you or me? ;)

\section{"Not all those who wander are lost"}

Working at the division of automatic control is a really pleasant experience. I am really grateful to all people in the group, not only for the small things, such as coffee breaks or planning meetings, but especially for the biggest thing of all, for making me feel at home, you are all like family! Unfortunately there is not enough room to personally thank everyone, but I would like to mention a few by name. Firstly, I would like to thank Johan Dahlin for helping me to keep one foot in reality when it has been the most stressful, both with meaningful and meaningless discussions (there is sausage in a vegetarian lasagna, right?). Sina Khoshfetrat Pakazad for always being the host of different kinds of activities and the unforgettable trip to Maui, but please, skip the poncho at the next canoeing trip! 
Zoran Sjanic for the (excessively) long discussions and for always being up for a beer, will you ever grow up? :) Manon Kok for always being there for discussions about the small and big things, I hope you will soon join for a ski or canoeing trip! Karl Granström for his abundance of junkmails, after works and skis, and possibly making me feel a bit too welcome at times! Isak Nielsen for being an awesome roommate, for bearing with me and all the fun trips! Hanna Nyqvist for entertaining me with all kinds of strange things and always having a smile on her face. André Carvalho Bittencourt for being my phadder, my first roommate and also a really good friend! Christian Andersson Naesseth, Christian Lyzell, Daniel Simon, Emre Özkan, Fredrik Lindsten, Henrik Ohlsson, Niklas Wahlström, Patrik Axelsson, Peter Rosander, Tianshi Chen, Tohid Ardeshiri (especially for bringing funny stories to the breakfast table) and Ylva Jung (and also for at least smiling at my jokes), for fun trips around the world! Finally, there is no question in my mind, that most of my multiple hangovers over the years here in Linköping are due to the bar-crawlers, Clas Veibäck, Marek Syldatk and Niclas Evestedt (Karl and Zoran), thank you very much! It is most appreciated :)

This work has been supported by the Vinnova Industry Excellence Center LINKSIC which is gratefully acknowledged! I am most grateful to Thor I. Fossen and Tor Arne Johansen at the Norwegian University of Science and Technology for their input regarding ship modeling and for supporting the model ship verification with their equipment and the wave basin. I would also like to thank Torgeir Wahl for his invaluable support during the wave basin tests.

I would deeply like to thank my family for still calling and wondering why I am working so late. Your care and interest in what I am doing is much appreciated, thank you for always being there for me! My friends outside the university might not have contributed to the thesis explicitly, but the support you give me by your friendship is invaluable, thank you for being awesome, you know who you are!

Finally, but not the least, I would like to thank my army of proof-readers, Gustaf Hendeby, Isak Nielsen, Hanna Nyqvist, Johan Dahlin, Manon Kok, and Ylva Jung. You are (is?;)) amazing, without you, this thesis would be a wreck! I would also like to thank Gustaf Hendeby for helping me with $\mathrm{LT}_{\mathrm{E}} \mathrm{X}$-related issues while writing my thesis!

"they say, if you travel far enough, you will find yourself"

I might not have found myself yet, but at least I have had an awesome journey and found a lot of friends! 


\section{Contents}

$\begin{array}{ll}\text { Notation } & \text { Xv }\end{array}$

1 Introduction $\quad 1$

1.1 Research Motivation . . . . . . . . . . . . . . . . . . . 1

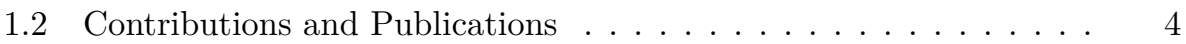

1.3 Thesis Outline ....................... 5

2 Selected Topics in System Identification $\quad 7$

2.1 Dynamic System . . . . . . . . . . . . . . . . . 8

2.2 The Concepts of System Identification . . . . . . . . . . . . . . . . . . 9

2.3 Parametric Models of Dynamic Systems . . . . . . . . . . . . . . 10

2.3.1 Transfer Function Model Structures . . . . . . . . . . . . . . 10

2.3.2 Linear Regression Model Structures . . . . . . . . . . . . . . . 11

2.3.3 State-space Model Structure . . . . . . . . . . . . . . . . . . . 12

2.4 Parameter Estimation Methods . . . . . . . . . . . . . . . 12

2.4.1 Prediction-Error Method . . . . . . . . . . . . . 12

2.4.2 Correlation Method - Instrumental Variable Method . . . . 13

2.5 Structural Identifiability of Linear Systems . . . . . . . . . . . . 15

3 Introduction to Ship Modeling $\quad 19$

3.1 Rigid-body Dynamics . . . . . . . . . . . . . . . . 20

3.1.1 Reference Frames and Reference Points . . . . . . . . . . 20

3.1 .2 Kinematics . . . . . . . . . . . . . . 22

3.1 .3 Kinetics . . . . . . . . . . . . . . . . . 23

3.2 Forces and Moments Due to Water Interaction . . . . . . . . . . 24

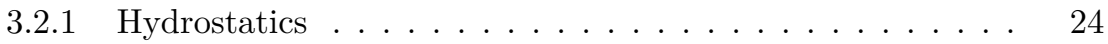

3.2.2 Maneuvering Hydrodynamics . . . . . . . . . . . . . 26

3.3 Actuators . . . . . . . . . . . . . . . . . 27

3.3 .1 Rudder . . . . . . . . . . . . . . . . . . . . . . . . . . . . .

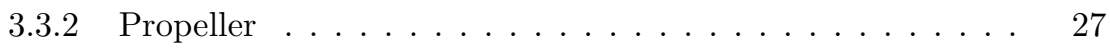

3.4 Environmental Disturbances . . . . . . . . . . . . . . 27

3.5 Surge-Sway-Roll-Yaw Maneuvering Model . . . . . . . . . . . . . . 30

3.5.1 Non-minimum Phase Behavior . . . . . . . . . . . . . 32 


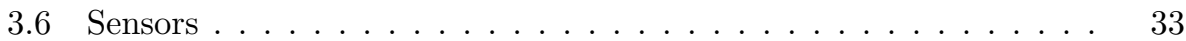

3.6.1 Inertial Measurement Unit . . . . . . . . . . . . . . . . 33

4 Graybox Modelling of a Second-order Subsystem 35

4.1 The Force-Mass Ambiguity . . . . . . . . . . . . . . . . . . 35

4.2 Approximate Model of a Pendulum . . . . . . . . . . . . . 36

4.2.1 Identifiability of the Approximate Model . . . . . . . . . 40

4.3 A Limited Sensor Approach - The Input Model . . . . . . . . . . . 44

4.3 .1 Identifiability of the Input Model . . . . . . . . . . . . . . 45

4.3.2 Model Analysis - A Closed-loop Viewpoint . . . . . . . . . 46

4.3.3 Discretization and Connection to the Physical Parameters. 48

4.3.4 Obeying Nonlinear Parameterization Using Constraints . . 49

4.4 Multi-Stage Method . . . . . . . . . . . . . . . . . . 51

4.4.1 Linear Criterion with Linear Constraints . . . . . . . . . 52

4.5 Experimental Verification . . . . . . . . . . . . . . . 54

4.5 .1 Simulation Study . . . . . . . . . . . . . . . . . 54

4.5.2 An Inverted Pendulum System . . . . . . . . . . . . . . 59

4.5.3 Summary of Experimental Studies . . . . . . . . . . . . 63

5 Estimation of a Ship's Roll Dynamics $\quad 65$

5.1 Model Derivation - A Limited Sensor Approach . . . . . . . . . 66

5.1 .1 Center of Rotation . . . . . . . . . . . . . . . . 69

5.2 Model Analysis . . . . . . . . . . . . . . . . . . . . . 70

5.2.1 The Signals' Dependency on the Input $\delta \ldots \ldots \ldots$. . . 70

5.2 .2 Identification Issues - Correlation with $\tau \ldots \ldots \ldots \ldots . \ldots 72$

5.3 Identifiability of the Roll Dynamics Subsystem . . . . . . . . . . 74

5.3.1 Limitations Using a Single Dataset . . . . . . . . . . . . . 75

5.3 .2 Multi-dataset Identifiability . . . . . . . . . . . . . 76

5.4 Discretization Using Physical Parameters . . . . . . . . . . . . 78

5.5 A Joint Identification Approach . . . . . . . . . . . . . . . . 80

5.5.1 The Pendulum Problem Revisited - A Joint Approach . . . 80

5.5.2 Estimation of the Roll Dynamics Using the Joint Approach 81

5.5 .3 The Iterative Joint IV Method . . . . . . . . . . . . . 83

6 Model Ship Experimental Verification $\quad 87$

6.1 Hardware - Experiment Overview . . . . . . . . . . . . . . 88

6.2 Model Ship . . . . . . . . . . . . . . . . . . . . . . 92

6.2.1 Calculation of the Ship's Center of Gravity . . . . . . . . 93

6.3 Experiment Description . . . . . . . . . . . . . . . . . . 94

6.4 Analysis of Data . . . . . . . . . . . . . . . . . . . 96

6.5 Estimation Results Under Moderate Sea Conditions . . . . . . . . 98

6.5.1 Case 1 - Equal Load in Calibration and Loaded Datasets . 104

6.5.2 Cases 2 and 3 - Lighter Load in the Calibration Dataset . 108

7 Conclusions

A Calculating the Mass and Center of Mass Through Ballasting 115 
Bibliography 

Notation

\section{Abbreviations}

\begin{tabular}{cl}
\hline Abbreviation & Meaning \\
\hline CB & Center of Buoyancy, see Definition 3.4 in Section 3.1.1 \\
CF & Center of Flotation, see Definition 3.5 in Section 3.1.1 \\
CG & Center of Gravity, see Definition 3.6 in Section 3.1.1 \\
CM & Center of Mass \\
CR & Center of Rotation, see Definition 3.7 in Section 3.1.1 \\
CO & Center of Origin, see Definition 3.8 in Section 3.1.1 \\
CS & Center of Sensor, see Definition 3.9 in Section 3.1.1 \\
IMU & Inertial Measurement Unit Section 3.6.1 \\
LCG & Lateral Center of Gravity see Section 3.3.1 \\
NED & North East Down, a convention for coordinate systems \\
NWU & North West Up, a convention for coordinate systems \\
\hline
\end{tabular}

\section{Rigid-body - Reference Frames}

\begin{tabular}{cl}
\hline Frame & Description \\
\hline$b$-frame & Body-fixed reference frame, see Definition 3.1 \\
$n$-frame & World-fixed reference frame, see Definition 3.2 \\
$s$-frame & Body-fixed Sensor reference frame, see Definition 3.3 \\
\hline
\end{tabular}




\section{Rigid-body - Generalized Positions}

\begin{tabular}{cl}
\hline Variable & Description \\
\hline$x$ & Position relative the $n$-frame \\
$y$ & Position relative the $n$-frame \\
$z$ & Position relative the $n$-frame \\
$\phi$ & Roll angle relative the $n$-frame \\
$\theta$ & Pitch angle relative the $n$-frame \\
$\psi$ & Yaw angle relative the $n$-frame \\
\hline
\end{tabular}

\section{Rigid-body - Generalized Velocities}

\begin{tabular}{cl}
\hline Variable & Description \\
\hline$u$ & Surge speed, linear velocity in $x_{b}$-axis of the $b$-frame \\
$v$ & Sway speed, linear velocity in $y_{b}$-axis of the $b$-frame \\
$w$ & Heave speed, linear velocity in $z_{b}$-axis of the $b$-frame \\
$p$ & roll-rate, angular velocity around the $x_{b}$-axis of the $b$ - \\
& frame \\
$q$ & $\begin{array}{l}\text { pitch-rate, angular velocity around the } y_{b} \text {-axis of the } \\
b \text {-frame } \\
r\end{array}$ \\
& yaw-rate, angular velocity around the $z_{b}$-axis of the $b$ - \\
\hline
\end{tabular}

\section{Dynamic Systems and System Identification}

\begin{tabular}{cl}
\hline Variable & Description \\
\hline $\mathrm{q}$ & Shift operator, i.e. $y_{t} \mathrm{q}=y_{t+1}$ \\
$\mathrm{p}$ & Differentiation operator, i.e. $y(t) \mathrm{p}=\dot{y}(t)$ \\
$\vartheta$ & Parameters in estimation problems \\
$\vartheta_{p}$ & Physical Parameters in estimation problems \\
$\hat{y}_{t}(\vartheta)$ & The one-step-ahead predictor of $y_{t}$ \\
\hline
\end{tabular}




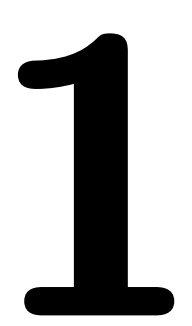

\section{Introduction}

In this thesis, we study the problem of estimating physical properties of mechanical systems and, in particular, of ships. This can be a difficult problem, partly due to the complex dynamics governing the interaction between the water and the ship and partly due to the limited set of sensors that is assumed to be available for the task. A complete model of a ship would be very complex and here we have instead chosen to work with approximate models that are simple but still accurate enough to capture some important properties of the system that is studied.

Science may be described as the art of systematic oversimplification.

- Karl Popper

This chapter will give a background and motivation for the work, together with a description of contributions and papers, and finally, the structure of the thesis will be explained.

\subsection{Research Motivation}

In automatic control, models are nowadays commonly used to increase performance or accuracy. This is essential, for instance, in model-based control where a poor model will affect the performance negatively (Skogestad and Postlethwaite, 2005), or in decision support systems where an error in the model might result in bad advices which might lead to the user eventually not trusting the system. Many mechanical systems, and certainly ships, have time-dependent physical properties. By estimating these properties online, a higher model accuracy can be achieved. Some examples are the change in center of mass due to passengers entering or leaving a car, the change of mass in an oil tanker due to oil being pumped into its tanks and the mass of a container ship being changed due to loading and unloading of containers as can be seen in Figure 1.1. 


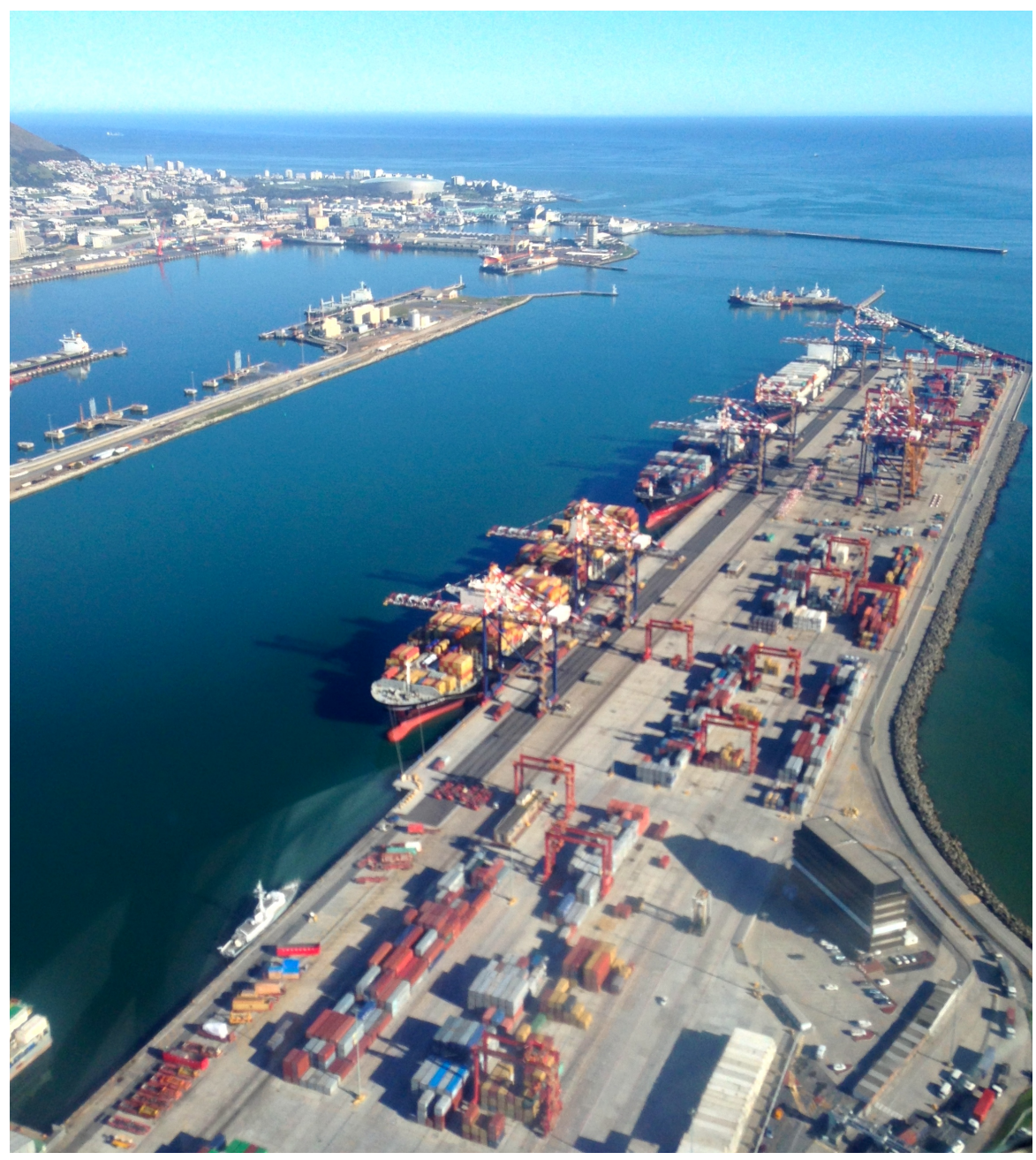

Figure 1.1: An aerial view of the Cape Town harbor showing three ships being tended to by the gantry cranes. A container ship can carry thousands of containers, each weighing several tons, and a significant part of the ship's weight is the cargo.

Naturally, these changing properties affect the dynamic behavior of the ships and the variation of certain properties might be safety critical. Fujiwara and Haraguchi (2005) investigate the dynamic influence of water flooding the car deck of roll-onroll-off passenger ships, such as the disaster of the ship Estonia in 1994. The authors show that the restoring properties, both the roll damping and the roll stiffness, change with the amount of water on deck. This a situation where online estimation of the parameters could be used to improve the accuracy of the model for later use in, for instance, a decision support system that could aid the crew to operate the vessel safely.

A ship's roll dynamics is very sensitive to changes in the loading conditions and 
a worst-case scenario is that the ship will capsize (Fossen, 2011; Tannuri et al., 2003). Moreover, a ship's response to waves is sensitive to the direction of the waves relative to the ship's heading (Faltinsen, 2005). For example, the ship's roll motion is more likely to be affected by waves approaching from the side than if the ship would face them with the bow first. Iseki and Terada (2001), Tannuri et al. (2003), Nielsen $(2007,2008)$, and Nielsen and Iseki $(2010,2011)$ study the problem of estimating the directional wave spectrum from ship motion. The wave spectrum can then be used to simulate a ship's response over time, to apply control or to suggest appropriate actions. This is basically an inverse problem where the spectrum of the input to the system is estimated and the result is dependent on the ship's mass. Inaccurate knowledge of the ship's mass, and thus of the model, might lead to an inaccurate wave spectrum, which in the end might lead to poor performance if the wave spectrum is used in the earlier mentioned control or decision support systems.

Perez (2005) investigates methods for ship roll stabilization or ship roll reduction systems using model-based control. The model used in the approach is a linear maneuvering model where the parameters are assumed to be known. The effects of model errors are not investigated, but due to the big change in the ship roll dynamics shown in, for instance, Fossen (2011) and Tannuri et al. (2003), there are reasons to believe that, for example, significant changes in mass will affect the ship dynamics and that online estimation of the mass would make it possible to increase the performance of the controller.

Actually, the mass, or inertia, is one of the most influential parameters in most mechanical systems. However, it is difficult to uniquely determine the mass in many cases due to inadequate measurements. For example, the acceleration $a$ of a particle with mass $m$, affected by the force $F$ and moving in a straight path is directly proportional to $F / m$. If the acceleration $a$ is measured, there are still multiple combinations of $F$ and $m$ that will solve the equation. Special experiments, where the forces acting on the system are known or can be measured, can be used to overcome this ambiguity, but doing special experiments is in many cases intractable. This is certainly true for the ship application that is studied here since it is difficult to measure all forces acting on the ship's hull.

Instead of focusing on a sensor-rich environment where all possible signals on a ship can be measured and a complete model of the ship is estimated, this thesis presents an approach where a model of a subsystem of the ship's dynamics is estimated using only a limited set of sensors. More specifically, it is assumed that only motion measurements from an inertial measurement unit (IMU) together with the rudder angle are available. Hence, direct measurements of the true inputs to the subsystem are not available, but the measurements indirectly contain information about the inputs and these indirect input measurements can be used as a substitute. The main benefit of this approach is that it can be applied easily without installing any expensive additional sensor systems on the ship. However, the limited measurement setup makes it critical to understand which parts of the roll dynamics can be estimated. 


\subsection{Contributions and Publications}

There are four main contributions in this thesis. The first contribution is the analysis of the identifiability and disturbance properties of the model with indirect input measurements presented in Chapter 4. The second contribution is the generalization of the indirect input measurement approach from Chapter 4 to ships and the derivation of the corresponding model from existing models in ship literature, see Chapter 5. The third contribution is the formulation of a closed-loop instrumental variable method for estimation of the unknown parameters in the models formulated in Chapters 4 and 5. The method builds on a graybox formulation and uses multiple datasets to overcome some identifiability issues, see Sections 4.4 and 5.5. The fourth contribution is the experimental work and the initial estimation results based on the collected ship data, see Chapter 6 .

The results in Chapter 4 have also been published in

J. Linder, M. Enqvist, F. Gustafsson, and J. Sjöberg. Identifiability of physical parameters in systems with limited sensors. In Proceedings of the 19th IFAC World Congress, Cape Town, South Africa, August 2014.

J. Linder, M. Enqvist, and F. Gustafsson. A closed-loop instrumental variable approach to mass and center of mass estimation using IMU data. In Proceedings of the 53rd IEEE Conference on Decision \& Control, Los Angeles, CA, USA, December 2014 (to appear).

The identifiability analysis is given in the first paper and the second paper describes the impact of disturbances and the use of the closed-loop instrumental variable approach to decrease the variance of the estimates.

Not included but published material are two papers related to Linder and Lindkvist (2011). These papers describe how optimization techniques can be used in decision support applications. Although not directly related, optimization is central in system identification and decision support systems are one of the intended application for the work in this thesis. The first paper is

J. Sjöberg, S. Lindkvist, J. Linder, and A. Daneryd. Interactive multiobjective optimization for the hot rolling process. In Proceedings of the 51st IEEE Conference on Decision \& Control, Maui, Hawaii, USA, December 2012.

and describes how the interactive multiobjective optimization framework developed in the thesis can be used to explore the solutions and learn about the hotrolling process. The second paper

J. Sjöberg, S. Lindkvist, J. Linder, and J. Öhr. Interactive multiobjective optimization for a grab-shift unloader crane. In Proceedings of the 19th IFAC World Congress, Cape Town, South Africa, August 2014.

describes how the interactive multiobjective optimization framework can be used to solve an optimal control problem to find a good trade-off between, for instance, minimum time and minimum energy. 


\subsection{Thesis Outline}

The theoretical foundation of this thesis is relying on two theories, system identification and ship modelling. Chapters 2 and 3 give a brief theoretical introduction to selected parts of these subjects.

Chapter 4 presents an introduction to the approximate model of the roll dynamics and the indirect input measurement framework together with an analysis of the identifibility properties of the model and the signals' dependencies on the disturbances. Furthermore, a sequential multi-stage instrumental variable method, that uses multiple datasets to overcome some identifiability issues, is introduced. Finally, an investigation of the suggested estimator's properties is presented in an experimental study.

In Chapter 5, it is shown that a well-established and more complete ship model can be used to derive a generalization of the approximate model, with more indirect input measurements and a few extra parameters. Furthermore, the analyses of Chapter 4 are extended to the generalized model. Finally, because of this extra complexity, a joint identification approach, which uses multiple datasets simultaneously and a graybox formulation, is presented.

Chapter 6 introduces the experimental setup used to collect data from a scale ship model for verification purposes. It also describes the estimation results obtained by applying the algorithm in Chapter 5 to the collected data.

Finally, in Chapter 7 the thesis is concluded and some ideas for future work are given. 



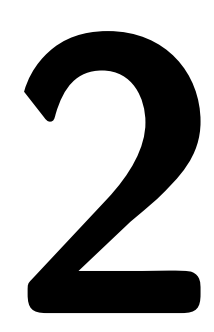

\section{Selected Topics in System Identification}

... causes and effects are discoverable, not by reason but by experience...

\section{- David Hume}

System identification is the science of building dynamical models based on measurements of signals in a system. The essence of system identification is the problem of induction, i.e. generalizing from a finite number of observations. In the nomenclature of system identification, the objective is to estimate, or infer, a connection between an input (cause) and the resulting output (effect). This inference results in a model (generalization) that is gained from measurements and knowledge of the system such as the structure of the dynamical model or values of some parameters (experience/previous knowledge).

A model is in this context never claimed to be the true description of the system, but rather a simplified mathematical description that is good enough for the intended application. The notion of a system is quite broad and it might, for instance, be a ship, the world's economy or even the human body.

To test the model's validity, it has to be falsifiable in some sense. The inferred model might, for instance, be validated with a second batch of measurements and be rejected if the outcome is too poor. In reality the outcome from the estimation and validation is used in an iterative process to deduce new theories and then the loop starts from the beginning.

This chapter is not an exhaustive description of system identification, but is rather supposed to give a brief introduction to certain topics needed to understand the work in this thesis. The focus is on estimation of parametric time-domain models. There are many approaches to system identification, and for a thorough treatment, see, for instance, Söderström and Stoica (1989), Schoukens and Pintelon (1991), Ljung (1999) or Pintelon and Schoukens (2012). 


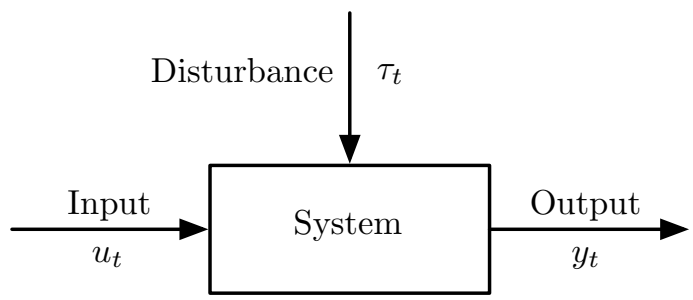

Figure 2.1: A schematic block representation of a system with input $u_{t}$, output $y_{t}$ and disturbance $\tau_{t}$.

\subsection{Dynamic System}

Figure 2.1 shows a schematic representation of a dynamic system. The system has two types of signals affecting it; inputs $u_{t}$ that are controllable and disturbances $\tau_{t}$ that are uncontrollable. The system also has a measurable output signal $y_{t}$ resulting from the inputs and disturbances. In contrast to a static system, a dynamic system has an internal memory and the output is not only dependent on the current inputs but also on previous inputs. In this thesis, the system is assumed to be causal, i.e. inputs and disturbances at time $t$ only affect the output at times $s>t$.

An example of a dynamic system in a control application can be seen in Figure 2.2 and in Example 2.1, the system concepts are related to a real world application, an autopilot for a ship.

\section{Example 2.1: A Dynamic System in a Control Application - Ship Autopilot $\longrightarrow$}

In an autopilot application, the goal is to steer the ship to a desired position and heading, using the available inputs to the system while the effects from disturbances are minimized. Figure 2.2 shows a ship controlled using a rudder and the speed of the motor. The dynamic system is the ship itself and its behavior defines the dynamics, for instance, the ship's response to a change in rudder angle. The input to the system is the rudder angle and the motor speed. The output $y_{t}$ is the position and possibly heading of the ship in relation to some global coordinate system, for instance, longitude, latitude and heading in relation to north. Furthermore, the signal $\tau_{t}$ represents the environmental disturbances affecting the ship. In this case, only the waves are considered. Since the input is the desired rudder angle and speed of the motor, the dynamics will also contain the rudder and motor dynamics. A complete model (if it were available) would be rather complex due to the ship's interaction with the environment. In a real application, simplified models are commonly used and are often sufficient for the application, see for instance, Källström et al. (1979) and Tzeng et al. (1999). 


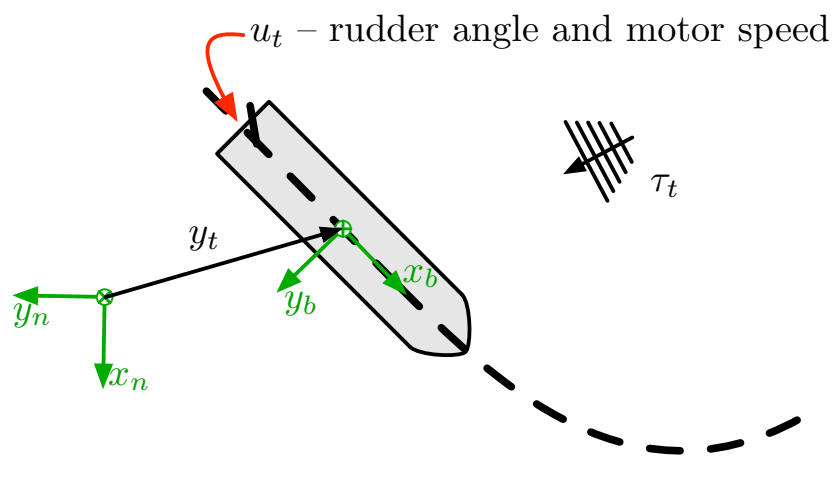

Figure 2.2: A ship can be seen as a dynamic system with inputs $u_{t}$ from the rudder angle and the motor speed and it is affected by the environmental disturbances $\tau_{t}$, here only the waves. The output $y_{t}$ is the position, and possibly heading, in a global coordinate system.

\subsection{The Concepts of System Identification}

The basis for all system identification is the observations in the form of a dataset. A dataset is a collection of $N$ data points denoted by

$$
Z_{N}=\left(y_{t}, u_{t}, o_{t}\right)_{t=1}^{N}
$$

where $y_{t}$ is the output, $u_{t}$ is the input and $o_{t}$ contains any other measured signals of interest, for example, measured disturbances, all at time $t$. In this thesis, parametric models are considered. A parametric model is parameterized by the vector $\vartheta$ and changing the values of the parameters will alter the properties of the model. In general, the model can be represented by the one-step-ahead predictor

$$
\hat{y}_{t}(\vartheta)=g\left(t, Z_{t-1}, u_{t} \mid \vartheta\right)
$$

which is here given as a general nonlinear filter based on previous data and the parameter $\vartheta$. The model is adapted to the data by estimating the parameters as

$$
\hat{\vartheta}=\underset{\vartheta \in \mathcal{D}}{\operatorname{argmin}} V_{N}\left(\vartheta, Z_{N}\right)
$$

where $V_{N}\left(\vartheta, Z_{N}\right)$ is a nonlinear criterion function that depends on the data and the model. The criterion is typically chosen to be a scalar measure of, for instance, the prediction error

$$
\varepsilon_{t}(\vartheta)=y_{t}-\hat{y}_{t}(\vartheta)
$$

A model is usually considered to be good if the criterion function is small when the model is applied to a set of observed data that was not used in the estimation. 


\subsection{Parametric Models of Dynamic Systems}

In a broad sense, a model is a concept that relates the variables of the estimation problem to each other. A model is thus abstract from this point of view. Here, we consider mathematical models. A model of a linear time-invariant system can be written

$$
y_{t}=G(\mathrm{q}) u_{t}+\tau_{t}=G(\mathrm{q}) u_{t}+H(\mathrm{q}) e_{t}
$$

where $\mathrm{q}$ is the forward shift operator, i.e. $\mathrm{q} y_{t}=y_{t+1}$, and $e_{t}$ is a white noise signal. A parametric model structure uses a finite number of parameters to specify the transfer functions $G(\mathrm{q})$ and $H(\mathrm{q})$. A candidate set of models is defined by

$$
y_{t}=G(\mathbf{q}, \vartheta) u_{t}+H(\mathbf{q}, \vartheta) e_{t}
$$

where $\vartheta \in D_{\mathcal{M}}$ and the challenge is to find which of these models that is most suitable. Comparison between different models in this set is usually done by comparing their ability to reproduce the output data, for instance, using prediction. The one-step-ahead predictor of $y_{t}$ is

$$
\hat{y}_{t}(\vartheta)=H^{-1}(\mathrm{q}, \vartheta) G(\mathrm{q}, \vartheta) u_{t}+\left[1-H^{-1}(\mathrm{q}, \vartheta)\right] y_{t}
$$

where outputs up to time $t-1$ and inputs up to time $t$ are used to predict the output at time $t$.

Proposing a model structure is by no means an easy task. At one end of the scale, there are first-principle (whitebox) model structures where everything is derived from physical laws and all needed parameters are known. On the other side of the scale are the blackbox model structures. The parameters in blackbox model structures have no physical meaning and they are used to tune the properties of the model to fit the data. In between these are the graybox model structures in which the parameters have physical interpretations, e.g. mass, density, length or resistance.

\subsubsection{Transfer Function Model Structures}

A typical example of a blackbox model structure is the general transfer function model structure (Ljung, 1999)

where

$$
A(\mathrm{q}, \vartheta) y_{t}=\frac{B(\mathrm{q}, \vartheta)}{F(\mathrm{q}, \vartheta)} u_{t}+\frac{C(\mathrm{q}, \vartheta)}{D(\mathrm{q}, \vartheta)} e_{t}
$$

$$
\begin{gathered}
B(\mathrm{q}, \vartheta)=\mathrm{q}^{-n_{k}}\left(b_{0}+b_{1} \mathrm{q}^{-1}+b_{2} \mathrm{q}^{-2}+\ldots+b_{n_{b}-1} \mathrm{q}^{-n_{b}+1}\right), \\
A(\mathrm{q}, \vartheta)=1+a_{1} \mathrm{q}^{-1}+a_{2} \mathrm{q}^{-2}+\ldots+a_{n_{a}} \mathrm{q}^{-n_{a}}
\end{gathered}
$$

and where the structures for $C(\mathrm{q}, \vartheta), D(\mathrm{q}, \vartheta)$ and $F(\mathrm{q}, \vartheta)$ are similar to the one for $A(\mathrm{q}, \vartheta)$ but not necessarily with the same number of parameters. This general structure is however too flexible in most cases and special cases of it are used instead. The special cases are obtained if some of the polynomials are set to unity and here we will list the ones that will be used later in this thesis in order of increasing complexity. 
One of the most commonly used model structures, the autoregressive with exogenous input (ARX) model structure

$$
A(\mathrm{q}, \vartheta) y_{t}=B(\mathrm{q}, \vartheta) u_{t}+e_{t}
$$

is obtained by setting $C(\mathrm{q}, \vartheta)=D(\mathrm{q}, \vartheta)=F(\mathrm{q}, \vartheta)=1$ in (2.8). The ARX model structure is especially attractive due to its simple structure and that the parameters can be found using fast estimation methods, see the next section. However, for a low model order, the noise model can be too restrictive and to increase flexibility in the noise model we can introduce a moving average (MA) part. The autoregressive moving average with exogenous input (ARMAX) model structure

$$
A(\mathrm{q}, \vartheta) y_{t}=B(\mathrm{q}, \vartheta) u_{t}+C(\mathrm{q}, \vartheta) e_{t}
$$

is obtained by setting $D(\mathrm{q}, \vartheta)=F(\mathrm{q}, \vartheta)=1$ in $(2.8)$ but has the drawback of a more computationally heavy estimation procedure. Finally, if $G(\mathrm{q}, \vartheta)$ and $H(\mathrm{q}, \vartheta)$ are parameterized individually, the Box-Jenkins (BJ) model structure

$$
y_{t}=\frac{B(\mathrm{q}, \vartheta)}{F(\mathrm{q}, \vartheta)} u_{t}+\frac{C(\mathrm{q}, \vartheta)}{D(\mathrm{q}, \vartheta)} e_{t}
$$

is obtained by setting $A(\mathrm{q}, \vartheta)=1$ in $(2.8)$.

The one-step-ahead predictor of (2.8) follows directly from (2.7) and is given by

$$
\hat{y}_{t}(\vartheta)=\frac{D(\mathrm{q}, \vartheta) B(\mathrm{q}, \vartheta)}{C(\mathrm{q}, \vartheta) F(\mathrm{q}, \vartheta)} u_{t}+\left[1-\frac{D(\mathrm{q}, \vartheta) A(\mathrm{q}, \vartheta)}{C(\mathrm{q}, \vartheta)}\right] y_{t}
$$

\subsubsection{Linear Regression Model Structures}

Let us consider the linear regression model structure that can be written on the form

$$
\hat{y}_{t}(\vartheta)=\varphi_{t}^{T} \vartheta
$$

where the elements of the regression vector $\varphi_{t}$ are known which means that the parameters can be estimated using a least-squares (LS) method. The ARX model structures is an example that can be written on this form. The one-step-ahead predictor of the ARX model can be written

$$
\hat{y}_{t}(\vartheta)=B(\mathbf{q}, \vartheta) u_{t}+[1-A(\mathbf{q}, \vartheta)] y_{t}
$$

and can be written on the linear regression model structure form by introducing

$$
\varphi_{t}=\left[-y_{t-1}, \ldots,-y_{t-n_{a}}, u_{t-n_{k}}, \ldots, u_{t-n_{b}+1-n_{k}}\right]^{T}
$$

and

$$
\vartheta=\left[a_{1}, \ldots, a_{n_{a}}, b_{0}, \ldots, b_{n_{b}-1}\right]^{T}
$$




\subsubsection{State-space Model Structure}

Another important representation of a dynamic system is the state-space model structure. It stores the memory of the system in a latent finite-dimensional vector where each element is called a state. The states contain the information about the effects of all previous inputs to the system. A state-space model structure is particularly useful in mechanical systems where the states usually have a physical representation, for instance, angular velocity or position.

A general nonlinear state-space model structure can be written

$$
\begin{aligned}
\dot{x}_{t} & =f\left(x_{t}, u_{t}, \tau_{t}, \vartheta\right) \\
y_{t} & =h\left(x_{t}, u_{t}, e_{t}, \vartheta\right)
\end{aligned}
$$

where $x_{t}$ is the state vector and $e_{t}$ is the measurements disturbance. For a linear time-invariant system, the linear state-space model structure

$$
\begin{aligned}
\dot{x}_{t} & =A(\vartheta) x_{t}+B(\vartheta) u_{t}+\tau_{t} \\
y_{t} & =C(\vartheta) x_{t}+D(\vartheta) u_{t}+e_{t}
\end{aligned}
$$

is commonly used and it can be transformed to a transfer function model structure

$$
y_{t}=\left(C(\vartheta)[\mathrm{s} I-A(\vartheta)]^{-1} B(\vartheta)+D(\vartheta)\right) u_{t}+\tilde{\tau}_{t}
$$

where $\tilde{\tau}_{t}$ represents the disturbance terms.

\subsection{Parameter Estimation Methods}

Once the model structure has been selected and the data have been collected, the model should be fitted to the data. Here we will describe two methods, the prediction-error method (PEM) and the instrumental variable (IV) method.

\subsubsection{Prediction-Error Method}

In the PEM the parameters are computed by minimizing the criterion function

$$
V_{N}\left(\vartheta, Z_{N}\right)=\frac{1}{N} \sum_{t=1}^{N} \ell\left(\varepsilon_{t}(\vartheta)\right)
$$

with respect to $\vartheta$ (Ljung, 1999). Here

$$
\varepsilon_{t}(\vartheta)=y_{t}-\hat{y}_{t}(\vartheta)
$$

is the prediction error. The scalar-valued function $\ell(\cdot)$ will here be assumed to be quadratic $\ell(x)=\frac{1}{2} x^{2}$. The parameters are thus found by computing

$$
\hat{\vartheta}=\underset{\vartheta}{\operatorname{argmin}} \frac{1}{N} \sum_{t=1}^{N} \frac{1}{2}\left(y_{t}-\hat{y}_{t}(\vartheta)\right)^{2}
$$

Note that this might be a nonconvex optimization problem for a general nonlinear one-step-ahead predictor. However, for certain model structures, (2.24) will simplify. In case that the one-step-ahead predictor can be written as a linear 
regression, the estimate of the parameters $\vartheta$ is given by solving the LS problem

where $\|x\|_{2}^{2}=x^{T} x$

$$
\hat{\vartheta}^{\mathrm{LS}}=\underset{\vartheta}{\operatorname{argmin}}\left\|Y_{N}-\Phi_{N} \vartheta\right\|_{2}^{2}
$$

$$
\Phi_{N}=\left[\varphi, \ldots, \varphi_{N}\right]^{T} \text { and } Y_{N}=\left[y_{1}, \ldots, y_{N}\right]^{T}
$$

For results on consistency, convergence and a deeper treatment of the PEM and the properties of its estimates, see Ljung (1999, Ch. 7).

\subsubsection{Correlation Method - Instrumental Variable Method}

The IV method is an alternative to the PEM. As the model (2.5) suggests, there are two terms contributing to $y_{t}$, one containing information about the interesting input-output relation and the second containing a contribution from the noise. An IV method is a correlation-based approach where the instrument vector $\zeta_{t}$ is used to extract the interesting information from the data. In principle, the interesting information, i.e. the parameters $\vartheta$, is found by requiring that

$$
\frac{1}{N} \sum_{t=1}^{N} \zeta_{t}\left(y_{t}-\varphi_{t}^{T} \vartheta\right)=0
$$

i.e. that the sample covariance between $\zeta_{t}$ and the prediction error should be zero. A good instrument should be correlated with the output $y_{t}$ but be uncorrelated with the disturbance $\tau_{t}$. This idea is generalized in the extended IV method, where the parameters are found by computing

$$
\hat{\vartheta}=\underset{\vartheta}{\operatorname{argmin}}\left\|Y_{N}-\Phi_{N} \vartheta\right\|_{Q}^{2}
$$

where $\|x\|_{Q}^{2}=x^{T} Q x, Q \geq 0$ is a weighting matrix,

$$
\Phi_{N}=\frac{1}{N}\left[\begin{array}{lll}
\zeta_{1} & \cdots & \zeta_{N}
\end{array}\right]\left[\begin{array}{c}
\bar{\varphi}_{1}^{T} \\
\vdots \\
\bar{\varphi}_{N}^{T}
\end{array}\right], Y_{N}=\frac{1}{N}\left[\begin{array}{lll}
\zeta_{1} & \cdots & \zeta_{N}
\end{array}\right]\left[\begin{array}{c}
\bar{y}_{1} \\
\vdots \\
\bar{y}_{N}
\end{array}\right],
$$

$\bar{y}_{t}=L(q) y_{t}, \bar{\varphi}_{t}^{T}=L(q) \varphi_{t}^{T}$ and $L(q)$ is a stable prefilter (Söderström and Stoica, 1989). The IV estimator will typically be consistent if the matrix

$$
\lim _{N \rightarrow \infty} \Phi_{N}
$$

has full rank and

$$
\lim _{N \rightarrow \infty} \frac{1}{N} \sum_{t=1}^{N} \zeta_{t} L(q) v_{t}=0,
$$

with probability 1. See, for instance, Söderström and Stoica (1983) or Ljung (1999) for more details.

Any instruments that satisfies the conditions in (2.30) will provide consistency, but the variance properties of the parameter estimator are greatly affected by the choice of instruments and prefilter, for more details see, for instance, Söderström and Stoica (1989), Ljung (1999) or Forssell (1999). 


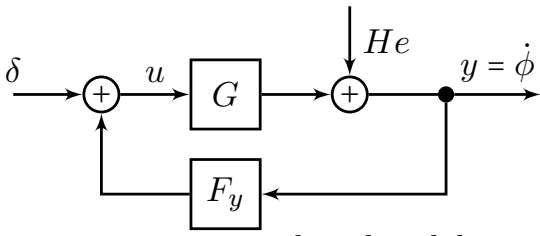

Figure 2.3: $A$ system is operating under closed loop conditions with $\delta$ as external reference.

\section{Instrumental Variable Method for Closed-loop Identification}

Figure 2.3 shows a system operating in closed loop and certain care has to be taken when the IV method is applied to such systems. The covariance properties of the closed-loop IV methods have been investigated in, for instance, Forssell and Chou (1998) and Gilson and Van den Hof (2005). Further insights about the choice of prefilter, the optimal variance instruments and approximatly optimal instruments for a closed-loop system, i.e. the system described in Figure 2.3, are given in, for instance, Gilson et al. (2011). These results are all based on using the noise model and an external reference signal $\delta$ instead of the input $u$ to create the optimal instruments.

Assume that the model is given on the form

$$
y_{t}=G\left(\mathbf{q}, \vartheta_{0}\right) u_{t}+H\left(\mathbf{q}, \vartheta_{0}\right) e_{t}=\varphi_{t}^{T} \vartheta_{0}+\tau_{t}
$$

where $u=\delta_{t}+F_{y}(\mathbf{q}) y_{t}$, that the regression vector is given by

$$
\varphi_{t}=\left[-y_{t-1}, \ldots,-y_{t-n_{a}}, u_{t-n_{k}}, \ldots, u_{t-n_{b}-n_{k}+1}\right]^{T}
$$

defined in (2.17) and that the disturbance is

$$
\tau_{t}=A\left(\mathrm{q}, \vartheta_{0}\right) H\left(\mathrm{q}, \vartheta_{0}\right) e_{t}
$$

where $A\left(\mathbf{q}, \vartheta_{0}\right)$ is the denominator of $G\left(\mathbf{q}, \vartheta_{0}\right)$ and it is assumed that $e_{t}$ is a white discrete-time zero-mean noise signal. Then the optimal prefilter is given by (Gilson et al., 2011)

$$
L\left(\mathrm{q}, \vartheta_{0}\right)=\frac{1}{A\left(\mathrm{q}, \vartheta_{0}\right) H\left(\mathrm{q}, \vartheta_{0}\right)}
$$

and the optimal instruments are given by the filtered noise-free regression vector

$$
\zeta_{t}=L\left(\mathbf{q}, \vartheta_{0}\right)\left[-\stackrel{\circ}{y}_{t-1}, \ldots,-\stackrel{\circ}{t}_{t-n_{a}}, \stackrel{\circ}{u}_{t-n_{k}}, \ldots, \stackrel{\circ}{t}_{t-n_{b}-n_{k}+1}\right]^{T}
$$

where $\dot{z}$ is the noise-free part of the signal $z$. However, these prefilters and instrument vectors are not implementable in reality since they require the knowledge of the true system and perfect measurements. Instead a variant of the iterative approach presented in Gilson et al. (2011) can be used. The method, described in Algorithm 2.1, is used to estimate $L(q)$ and create the instruments $\zeta_{t}$. In this method, the instrument vector is

$$
\zeta_{t}=L(q)\left[\hat{y}_{s, t-1} \cdots \hat{y}_{s, t-n_{y}}, \hat{u}_{s, t-n_{k}} \cdots \hat{u}_{s, t-n_{k}-n_{u}+1}\right]^{T}
$$

where $L(q)$ is a prefilter,

$$
\hat{y}_{s, t}=\frac{G(q, \hat{\vartheta})}{1-G(q, \hat{\vartheta}) F_{y}(q)} \delta_{t} \quad \text { and } \quad \hat{u}_{s, t}=\frac{1}{1-G(q, \hat{\vartheta}) F_{y}(q)} \delta_{t}
$$


are the simulated output and input, respectively, and $\hat{\vartheta}$ is an estimate of the parameters. The constants $n_{y}$ and $n_{u}$ are the number of time lags included in $\zeta_{t}$ for the output and the input, respectively, i.e. $n_{y}=0$ means that $\hat{y}_{s, t}$ is not included in $\zeta_{t}$. The prefilter is given by

$$
L(\mathrm{q}, \eta)=\frac{1}{\hat{H}(\mathrm{q}, \eta)}
$$

where $\hat{H}(\mathrm{q}, \hat{\eta})$ is an ARMA model estimated from the residual $\varepsilon_{t}=y_{t}-\varphi_{t}^{T} \hat{\vartheta}$.

\section{Algorithm 2.1 (Iterative method to decrease the variance of $\hat{\vartheta}$ )}

(A) Initialize:

(a) Set $L^{(0)}(q)=1$

(b) Set $\zeta_{t}^{(0)}=\left[\delta_{t} \cdots \delta_{t-n_{r, 0}+1}\right]^{T}$ where $n_{r, 0}$ is the number of time lags included

(c) Set $i=1$

(B) Estimate parameters:

(a) Estimate $\hat{\vartheta}^{(j)}$ using $(2.28)$ with $L^{(j-1)}(q)$ and $\zeta_{t}^{(j-1)}$

(b) Estimate the parameters $\hat{\eta}^{(j)}$ of the model $\hat{H}(q, \eta)$ from $\varepsilon_{t}=\hat{H}(q, \eta) \bar{e}_{t}$ where $\varepsilon_{t}=y_{t}-\varphi_{t}^{T} \hat{\vartheta}^{(j)}$

(c) Create the prefilter $L^{(j)}(q)=\frac{1}{\hat{H}\left(q, \hat{\eta}^{(j)}\right)}$

(d) Simulate $\hat{y}_{s, t}^{(j)}$ and $\hat{u}_{s, t}^{(j)}$ according to $(2.36)$ using $\hat{\vartheta}^{(j)}$ and create the instrument $\zeta_{t}^{(j)}$ according to $(2.35)$

(C) Terminate: Increase $j$ and go to Step B while $\left\|\hat{\vartheta}^{(j)}-\hat{\vartheta}^{(j-1)}\right\|_{2}+\left\|\hat{\eta}^{(j)}-\hat{\eta}^{(i-1)}\right\|_{2}$ is above a threshold or as long as a maximum number of iterations is not reached, return $\hat{\vartheta}=\hat{\vartheta}^{(j)}$ and terminate otherwise.

\subsection{Structural Identifiability of Linear Systems}

The subject of uniquely determining the parameters in a model structure has several aspects worth considering (Bellman and Åström, 1970; Ljung and Glad, 1994). This section is focused on the structural identifiability of linear systems. The model structure might very well be nonlinearly parameterized but the system is assumed to be linear in the input. The outcome of the analysis is a qualitative measure, i.e. if it is possible (simply yes or no) to uniquely determine the parameters of a model structure, without giving a measure of the quality of the estimates. In the general case, a linear model parameterized with parameter vector $\vartheta \in \mathbb{R}^{n_{\vartheta}}$ can be written in the state space form

$$
\begin{aligned}
& \dot{x}=A(\vartheta) x+B(\vartheta) u \\
& y=C(\vartheta) x
\end{aligned}
$$


where $x \in \mathbb{R}^{n}$ is the state vector, $u \in \mathbb{R}^{m}$ is the input, $y \in \mathbb{R}^{n_{p}}$ is the output and the matrices are of suitable sizes. Note that the parameterization might be nonlinear even though the system is linear in its state and input. Identifiability can be seen as observability of the extended nonlinear system

$$
\begin{aligned}
{\left[\begin{array}{c}
\dot{x} \\
\dot{\vartheta}
\end{array}\right] } & =\left[\begin{array}{c}
f(x, u, \vartheta) \\
0
\end{array}\right]=g^{0}(x, \vartheta)+g(x, \vartheta) u \\
y & =h(x, \vartheta)
\end{aligned}
$$

where $g^{0}(x, \vartheta), g(x, \vartheta)$ and $h(x, \vartheta)$ are assumed to be rational in the extended states (Walter, 1982). To show observability for this nonlinear system (identifiability for the linear system), the system of equations

$$
\begin{aligned}
y_{1}^{(0)} & =h_{1}^{(0)}(x, \vartheta) \\
y_{1}^{(1)} & =h_{1}^{(1)}\left(x, \vartheta, u_{1}, \ldots, u_{m}\right) \\
\vdots & \vdots \\
y_{1}^{(K)} & =h_{1}^{(K)}\left(x, \vartheta, u_{1}, \ldots, u_{1}^{(K-1)}, \ldots, u_{m}, \ldots, u_{m}^{(K-1)}\right) \\
y_{n_{p}}^{(0)} & =h_{n_{p}}^{(0)}(x, \vartheta) \\
y_{n_{p}}^{(1)} & =h_{n_{p}}^{(1)}\left(x, \vartheta, u_{1}, \ldots, u_{m}\right) \\
\vdots & \\
y_{n_{p}}^{(K)} & =h_{n_{p}}^{(K)}\left(x, \vartheta, u_{1}, \ldots, u_{1}^{(K-1)}, \ldots, u_{m}, \ldots, u_{m}^{(K-1)}\right)
\end{aligned}
$$

has to be solved for $x$ and $\vartheta$, where $y, u$ and their time derivatives are assumed to be known (Diop and Fliess, 1991). Here, the notation $(\cdot)^{(k)}$ means the $k^{\text {th }}$ derivative of $(\cdot)$ with respect to time. For a general nonlinear system, there is no upper limit on $K$ and it might be infeasible to solve this system of equations or difficult to analyze the result. Instead of solving the system of equations, it can be linearized around the extended states and an analysis can be performed on the Jacobian (Hermann and Krener, 1977)

$$
\mathcal{O}\left(x, \vartheta, u_{1}, \ldots, u_{p}^{(K-1)}\right)=\left[\begin{array}{c}
\frac{\partial h_{1}^{(0)}(x, \vartheta)}{\partial(x, \vartheta)} \\
\frac{\partial h_{1}^{(1)}\left(x, \vartheta, u_{1}, \ldots, u_{m}\right)}{\partial(x, \vartheta)} \\
\vdots \\
\frac{\partial h_{1}^{(K)}\left(x, \vartheta, u_{1}, \ldots, u_{1}^{(K-1)}, \ldots, u_{m}, \ldots, u_{m}^{(K-1)}\right)}{\partial(x, \vartheta)} \\
\vdots \\
\frac{\partial h_{n}^{(0)}(x, \vartheta)}{\partial(x, \vartheta)} \\
\frac{\partial h_{n_{p}}^{(1)}\left(x, \vartheta, u_{1}, \ldots, u_{m}\right)}{\partial(x, \vartheta)} \\
\vdots \\
\vdots \\
\frac{\partial h_{n_{p}}^{(K)}\left(x, \vartheta, u_{1}, \ldots, u_{1}^{(K-1)}, \ldots, u_{m}, \ldots, u_{m}^{(K-1)}\right)}{\partial(x, \vartheta)}
\end{array}\right]
$$

which hereafter will be called the extended observability matrix (for simplicity denoted $\mathcal{O}$ ). The system is locally weakly identifiable if $\mathcal{O}$ satisfies an observability rank condition (Hermann and Krener, 1977). 
In Sections 4 and 5 of Anguelova (2007), it is shown that under the assumption that the system equations, i.e. $h(x, \vartheta), g^{0}(x, \vartheta)$ and $g(x, \vartheta)$ in $(2.39)$, are rational functions in the states and parameters, it is enough to include the first $K=n+n_{\vartheta}-1$ derivatives of $h_{j}(x, \vartheta), j=1, \ldots, n_{p}$, with respect to time. It is also shown that if $\operatorname{rank}(\mathcal{O})=n_{q}<n+n_{\vartheta}$, it is enough to form $\mathcal{O}$ using the first $n_{q}-1$ derivatives with respect to time. This is useful if the dimension of the extended system is large since the complexity of the rows of $\mathcal{O}$ increases for high values of $K$. If the rank is less than $n+n_{\vartheta}$, one can exclude columns from the observability matrix one-by-one to identify the unobservable parameters or states since excluding a parameter in the extended system is equivalent to dropping the corresponding column in $\mathcal{O}$. See Anguelova (2007) for a thorough discussion about identifiability of systems that are rational in the extended state vector. 



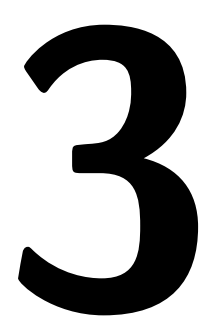

\section{Introduction to Ship Modeling}

This chapter is an introduction to modeling of ships and the environmental forces acting on them. The interaction between the ship and the environment makes the modeling of forces and moments acting on the ship very complex. The model type used is dependent on its field of application and the major difference is in how the theories deal with hydrodynamic phenomenas. The models are divided into seakeeping theory, i.e. motion due to waves of a ship with constant speed and course, and maneuvering theory, i.e. motion due to control inputs of a ship in calm water. Classically, the complete response of the ship is given by motion superposition of these models. An alternative is to use the unified theory that combines components from both theories with force superposition (Perez, 2005).

In this thesis, the focus is on separating the motion induced by actuators from the total motion without modeling the motions induced by the environmental disturbances. In this chapter, we will hence concentrate on specific parts of maneuvering theory models. Additionally, environmental disturbances will briefly be discussed with the aim of understanding the properties of these disturbances. For a more complete treatment covering modeling of environmental disturbances and ships, also covering seakeeping and unified theory, see for instance, Newman (1977), Faltinsen (1990), Journée and Massie (2001), Perez (2005) and Fossen (2011).

Here, we will consider models suitable for system identification and work with a simplified version of the model

$$
\begin{aligned}
\dot{\boldsymbol{\eta}} & =\boldsymbol{J}(\boldsymbol{\eta}) \boldsymbol{\nu} & & - \text { Kinematics: Section } 3.1 .2 \\
\boldsymbol{M}_{R B} \dot{\boldsymbol{\nu}}+\boldsymbol{C}_{R B}(\boldsymbol{\nu}) \boldsymbol{\nu} & =\boldsymbol{\tau} & & - \text { Kinetics: Section 3.1.3 }
\end{aligned}
$$

that was first described in Fossen (1991). The notation will be described in the following sections. The forces acting on the system are

$$
\boldsymbol{\tau}=\underbrace{\boldsymbol{\tau}_{\text {hydro }}}_{\text {Section 3.2 }}+\underbrace{\boldsymbol{\tau}_{\text {actuators }}}_{\text {Section 3.3 }}+\underbrace{\boldsymbol{\tau}_{\text {environmental }}}_{\text {Section } 3.4}
$$

and they will be described in the indicated sections. Furthermore, the ship's state will be measured by sensors described by

$$
y=\boldsymbol{h}(\boldsymbol{\eta}, \boldsymbol{\nu})+e \quad-\text { Sensors: Section } 3.6
$$




\subsection{Rigid-body Dynamics}

In this thesis, the ship is assumed to be a non-flexible structure. This is a common assumption and for a treatment of flexible structures, see for instance, Drummen (2008). This is an idealization, but allows us to use rigid-body dynamics, which is the study of motion of a rigid-body under influence from external forces. Dynamics can straightforwardly be divided into kinematics, the geometric description of the motion, and kinetics, the effect of forces acting on the body, see for instance, Thornton and Marion (2004), Spong and Hutchinson (2005) or Fossen (2011).

The pose of the ship can be described using three coordinates for the position, three coordinates for the rotation and the motion of the ship is fully specified by the pose and its time derivatives. These are called the generalized position and the generalized velocity, respectively, and we will get back to them shortly.

\subsubsection{Reference Frames and Reference Points}

A convenient way of relating the generalized positions and velocities is by the means of reference frames, one body-fixed frame attached to the ship and one inertial frame. These will be called the $b$-frame and the $n$-frame, respectively, and are defined according to Definition 3.1 and Definition 3.2. The sensor $s$-frame is also defined in Definition 3.3 for later use.

Definition 3.1 ( $b$-frame) The $b$-frame has its origin $o_{b}$ fixed to the ship, the $x_{b}$ axis pointing towards the bow (forward), the $y_{b}$-axis pointing to starboard (right) and the $z_{b}$-axis pointing downwards, see Figure 3.1 .

Definition 3.2 ( $n$-frame) The $n$-frame has its origin $o_{n}$ fixed to earth. The $n-$ frame is a north-east-down (NED) coordinate system, i.e. the $x_{n}$-axis pointing towards the north, the $y_{n}$-axis pointing towards the east and the $z_{n}$-axis pointing towards the center of the earth, see Figure 3.1(a). Note that this is not truly an inertial frame, but for a ship moving locally it is a reasonable assumption (Fossen, 2011).

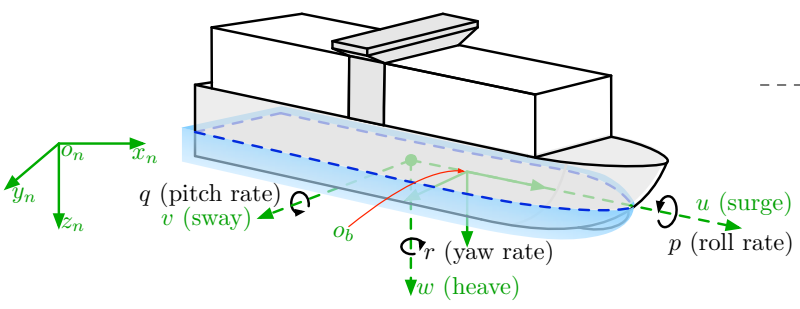

(a) Reference frames

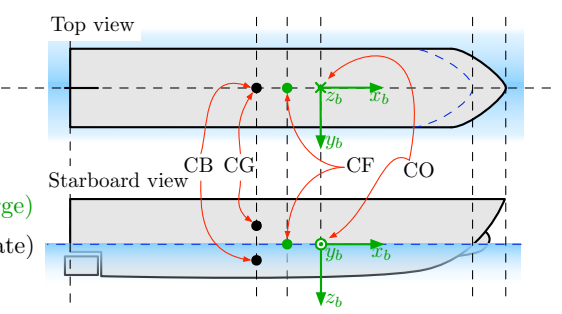

(b) Reference points

Figure 3.1: The reference frames and reference points in Definitions 3.1-3.9. 

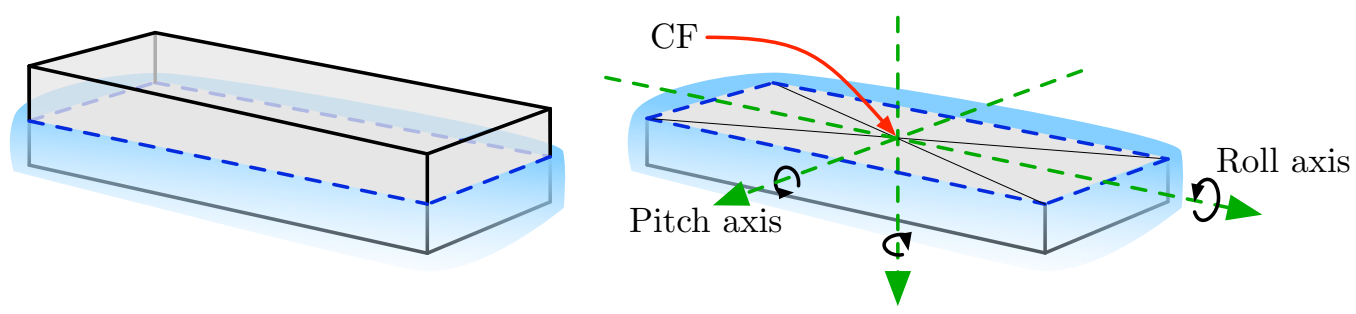

Figure 3.2: A rectangular block is floating in water. The water plane is the intersection between the block and the water surface when the block it at rest. The centroid, or geometric center, is in this case in the center of the block.

Definition 3.3 ( $s$-frame) The $s$-frame has its origin $o_{s}$ fixed to the ship, the $x_{s}-$ axis pointing towards the bow (forward), the $y_{s}$-axis pointing to port (left) and the $z_{s}$-axis pointing upwards.

In order to further simplifying the modeling, certain body-fixed reference points can be defined. These are defined as a vector $\boldsymbol{r}_{i}=\left[x_{i}, y_{i}, z_{i}\right]^{T}$ in the $b$-frame. The significance of these points will be clear in the coming sections. Here, they are simply enumerated in Definitions $3.4-3.9$.

Definition 3.4 (Center of Buoyancy (CB)) The center of buoyancy, described by $\boldsymbol{r}_{b}$, is the center of mass for the water displaced by the ship, see Figure 3.1(b). Note that this point is dependent on the hull shape and the attitude of the ship, see also Section 3.2.1.

Definition 3.5 (Center of Flotation (CF)) The center of flotation, described by $\boldsymbol{r}_{f}$, is the centroid (geometric center) of the of water plane in calm water, see Figure 3.1(b). An example for a rectangular block can be seen in Figure 3.2.

Definition 3.6 (Center of Gravity (CG)) Here it is assumed that the ship is operating in a constant and homogenous gravity field. Hence, the center of gravity, described by $\boldsymbol{r}_{g}$, coincides with the ship's center of mass (CM), see Figure 3.1(b).

Definition 3.7 (Center of Rotation (CR)) The center of rotation is the instantaneous point around which the ship rotates. Note that for a rigid-body in free space the rotation center will coincide with its CG. However, for a ship the center of rotation is shifted due to the added mass (read added inertia) due to its motion through water, see for instance Balcer (2004). In this thesis, however, the center of rotation is assumed to be fixed and equal to the $\mathrm{CF}$.

Definition 3.8 (Center of Origin (CO)) The center of origin, is the origin $o_{b}$ of the $b$-frame.

Definition 3.9 (Center of Sensor (CS)) The center of sensor, with the origin $o_{s}$, located with $\boldsymbol{r}_{s}$, is the center of the IMU described in Section 3.6.1. See also Definition 3.3. 
Table 3.1: The generalized positions and velocities used to describe the ship's position and orientation. Note that $\phi, \theta$ and $\psi$ are Euler angles and that the $z y x$ convention is used (Fossen, 2011).

\begin{tabular}{cccc}
\hline \multirow{2}{*}{ Generalized } & & Position & Velocity \\
\hline \multirow{4}{*}{ Linear } & & $\boldsymbol{\eta}$ & $\boldsymbol{\nu}$ \\
& Surge & $x$ & $u$ \\
& Sway & $y$ & $v$ \\
& Heave & $z$ & $w$ \\
\hline \multirow{4}{*}{ Angular } & Roll & $\phi$ & $p$ \\
& Pitch & $\theta$ & $q$ \\
& Yaw & $\psi$ & $r$ \\
\hline
\end{tabular}

\subsubsection{Kinematics}

The kinematics can now be derived by relating the generalized position

$$
\boldsymbol{\eta}=\left[\boldsymbol{p}^{T}, \boldsymbol{\Theta}^{T}\right]^{T}=[x, y, z, \phi, \theta, \psi]^{T}
$$

which is the translation and rotation of the $b$-frame with respect to the $n$-frame expressed in the $n$-frame, and the generalized velocity

$$
\boldsymbol{\nu}=\left[\boldsymbol{v}^{T}, \boldsymbol{\omega}^{T}\right]^{T}=[u, v, w, p, q, r]^{T}
$$

which is the body-fixed linear velocities and body-fixed angular velocities expressed in the $b$-frame. Note that if the forward speed $u$ is assumed to be constant, it is denoted by $U$. See Table 3.1 for a description of the variables and Figure 3.1(a) for a geometric description.

As already mentioned, the generalized positions and the generalized velocities have a geometric relation that is independent of the forces acting on the rigid-body. The relation is

$$
\dot{\boldsymbol{\eta}}=\boldsymbol{J}(\boldsymbol{\eta}) \boldsymbol{\nu}
$$

where

$$
\boldsymbol{J}(\boldsymbol{\eta})=\left[\begin{array}{cc}
\boldsymbol{R}(\Theta) & \mathbf{0}_{3 \times 3} \\
\mathbf{0}_{3 \times 3} & \boldsymbol{T}(\boldsymbol{\Theta})
\end{array}\right]
$$

is a transformation matrix with the rotation matrix

$$
\boldsymbol{R}(\boldsymbol{\Theta})=\left[\begin{array}{ccc}
c_{\psi} c_{\theta} & -s_{\psi} c_{\phi}+c_{\psi} s_{\theta} s_{\phi} & s_{\psi} s_{\phi}+c_{\psi} c_{\phi} s_{\theta} \\
s_{\psi} c_{\theta} & c_{\psi} c_{\phi}+s_{\phi} s_{\theta} s_{\psi} & -c_{\psi} s_{\theta}+s_{\theta} s_{\psi} c_{\phi} \\
-s_{\theta} & c_{\theta} s_{\phi} & c_{\theta} c_{\phi}
\end{array}\right]
$$

describing the relation between the linear speeds in the $b$-frame and the linear speeds in the $n$-frame (Fossen, 2011, Ch. 2). Here, $\mathbf{0}_{x \times y}$ is a $x$ by $y$ matrix with all elements equal to zero, $s_{x}=\sin (x), c_{x}=\cos (x)$ and $t_{x}=\tan (x)$. Furthermore, the transformation matrix

$$
\boldsymbol{T}(\boldsymbol{\Theta})=\left[\begin{array}{ccc}
1 & s_{\phi} t_{\theta} & c_{\phi} t_{\theta} \\
0 & c_{\phi} & -s_{\phi} \\
0 & s_{\phi} / c_{\theta} & c_{\phi} / c_{\theta}
\end{array}\right]
$$


describes the angular velocity transformation from $b$-frame to $n$-frame (Fossen, 2011, Ch. 2). Note that $\phi, \theta$ and $\psi$ are Euler angles and that the rotation matrix has been created by multiplying the principal rotation matrices for the $z, y$ and $x$ axes in that order, see, for instance, Thornton and Marion (2004), Spong and Hutchinson (2005) or Fossen (2011), for a thorough treatment of transformation matrices.

\subsubsection{Kinetics}

The kinetics, i.e. effects of forces acting on the rigid-body, can be derived, for instance, by using Euler-Lagrange equations or the Newton-Euler formulation. Here, the derivation is omitted and the interested reader is referred to Fossen (1991), Thornton and Marion (2004), Perez (2005), Spong and Hutchinson (2005) or Fossen (2011). The kinetics can be expressed as

$$
\boldsymbol{M}_{R B} \dot{\boldsymbol{\nu}}+\boldsymbol{C}_{R B}(\boldsymbol{\nu}) \boldsymbol{\nu}=\boldsymbol{\tau}
$$

which is the vectorial representation described in Fossen (1991). Here $\boldsymbol{M}_{R B}$ is the rigid-body inertia matrix, $\boldsymbol{C}_{R B}(\boldsymbol{\nu})$ represents the centripetal and Coriolis terms, and $\tau$ is a vector of generalized forces, all expressed in the $b$-frame.

The matrices $\boldsymbol{M}_{R B}$ and $\boldsymbol{C}_{R B}(\boldsymbol{\nu})$ are calculated for the fixed point CO that does not necessarily have to coincide with the CG. This is convenient since the CG will move with different loading conditions. The vector relating $\mathrm{CO}$ and $\mathrm{CG}$ is denoted $\boldsymbol{r}_{g}$ and is defined in Definition 3.6. Here, the skew-symmetric matrix

$$
\boldsymbol{S}(\boldsymbol{x})=-\boldsymbol{S}(\boldsymbol{x})^{T}=\left[\begin{array}{ccc}
0 & -x_{3} & x_{2} \\
x_{3} & 0 & -x_{1} \\
-x_{2} & x_{1} & 0
\end{array}\right], \quad \boldsymbol{x}=\left[\begin{array}{l}
x_{1} \\
x_{2} \\
x_{3}
\end{array}\right]
$$

is used to simplify notation. Note that, $\boldsymbol{S}(\boldsymbol{x}) \boldsymbol{y}=\boldsymbol{x} \times \boldsymbol{y}$, i.e. the vector cross-product, see Fossen (2011, p. 20). For motion about CO the inertia matrix is given by

$$
\boldsymbol{M}_{R B}=\left[\begin{array}{cc}
\bar{M} \boldsymbol{I}_{3 \times 3} & -\bar{M} \boldsymbol{S}\left(\boldsymbol{r}_{g}\right) \\
\bar{M} \boldsymbol{S}\left(\boldsymbol{r}_{g}\right) & \boldsymbol{I}_{g}-\bar{M} \boldsymbol{S}^{2}\left(\boldsymbol{r}_{g}\right)
\end{array}\right]=\left[\begin{array}{cc}
\bar{M} \boldsymbol{I}_{3 \times 3} & -\bar{M} \boldsymbol{S}\left(\boldsymbol{r}_{g}\right) \\
\bar{M} \boldsymbol{S}\left(\boldsymbol{r}_{g}\right) & \boldsymbol{I}_{b}
\end{array}\right]
$$

where $\boldsymbol{I}_{x \times x}$ is the $x$ by $x$ identity matrix, $\bar{M}$ is the total mass of the ship including additional loads such as cargo, $\boldsymbol{I}_{b}$ is the inertia matrix around $\mathrm{CO}$ and $\boldsymbol{I}_{g}$ is the inertia matrix about CG. The inertia matrix has an unique representation and satisfies $\boldsymbol{M}_{R B}=\boldsymbol{M}_{R B}^{T}>0$ and $\dot{\boldsymbol{M}}_{R B}=\mathbf{0}_{6 \times 6}$. The matrix $\boldsymbol{C}_{R B}(\boldsymbol{\nu})$ is however not unique due to the cross-terms, for example, $K_{u r} u r=C_{1}(u) r=C_{2}(r) u$. One representation is

$$
\boldsymbol{C}_{R B}(\boldsymbol{\nu})=\left[\begin{array}{cc}
\bar{M} \boldsymbol{S}(\boldsymbol{\omega}) & -\bar{M} \boldsymbol{S}(\boldsymbol{\omega}) \boldsymbol{S}\left(\boldsymbol{r}_{g}\right) \\
\bar{M} \boldsymbol{S}\left(\boldsymbol{r}_{g}\right) \boldsymbol{S}(\boldsymbol{\omega}) & -\boldsymbol{S}\left(\boldsymbol{I}_{b} \boldsymbol{\omega}\right)
\end{array}\right]
$$

that is independent of the body-fixed linear velocities $\boldsymbol{v}$. Which representation that is suitable depends on the intended use of the model, see Fossen (2011, p. 53-56). 


\subsection{Forces and Moments Due to Water Interaction}

The forces acting on a ship due to interaction with the water are very complex. A common assumption is that the forces due to different phenomena can be modeled individually and that linear superposition can be used (Faltinsen, 1990, 2005). In this thesis, the forces acting on the ship are assumed to be given by a general nonlinear function which will here be expressed by a series expansion. The forces are usually split into hydrostatics, which is the restoring forces, and hydrodynamics, which is the added inertia and damping. The forces and moments in (3.2) due to interaction with water are assumed to be given by

$$
\tau_{\text {hydro }}=\overbrace{-M_{A} \dot{\nu}-C_{A}(\nu) \nu-D(\eta, \nu)}^{\text {Hydrodynamics }} \overbrace{-\boldsymbol{g}(\boldsymbol{\eta})}^{\text {Hydrostatics }}=-\boldsymbol{f}(\boldsymbol{\eta}, \dot{\boldsymbol{\nu}}, \boldsymbol{\nu})-\boldsymbol{g}(\boldsymbol{\eta})
$$

which combined with the rigid-body kinetics gives the resulting nonlinear model

$$
\boldsymbol{M} \dot{\boldsymbol{\nu}}+\boldsymbol{C}(\boldsymbol{\nu}) \boldsymbol{\nu}+\boldsymbol{D}(\boldsymbol{\eta}, \boldsymbol{\nu})+\boldsymbol{g}(\boldsymbol{\eta})=\boldsymbol{\tau}_{\text {actuators }}+\boldsymbol{\tau}_{\text {environmental }}
$$

that is of mass-spring-damper type and where the coefficients are assumed to be constant. Here, the inertia and Coriolis matrices are

$$
M=M_{R B}+M_{A}
$$

and

respectively.

$$
C(\nu)=C_{R B}(\nu)+C_{A}(\nu)
$$

\subsubsection{Hydrostatics}

The restoring forces of a vessel are given by the buoyancy and gravitationally forces acting on the ship. At equilibrium, all floating bodies will have the $\mathrm{CG}$ and $\mathrm{CB}$ on a vertical line. Note that for most stable floating objects, such as ships, the CG will be above the CB (Lautrup, 2004). The restoring torque is expressed in terms of the so called righting arm. In order to understand what the righting arm is, consider Figure 3.3 that shows a box-shaped ship seen from the front. When the ship rolls around the $\mathrm{CF}$, the rolling motion will result in the $\mathrm{CB}$ being shifted away from the ship's vertical axis. This shift, from the old to the new CB, creates a restoring torque that can be written as

$$
\rho \nabla \overline{G Z}(\phi)
$$

where $\nabla$ is the displaced water volume, $g$ is the acceleration of gravity and $\rho$ is the density of water. The righting arm $\overline{G Z}(\phi)$ is the distance between the center of gravity $\mathrm{CG}$ and a vertical line through the new $\mathrm{CB}$, see Figure 3.3.

It can be shown that for small roll angles $\phi$, the righting arm can be described using the metacenter $M_{T}$. The metacenter $M_{T}$ is the point at which a vertical line through a new CB crosses the line that passes through the old CB and the CG when the ship is tilted an infinitesimal small roll angle. Note that $M_{T}$ is a constant and purely geometric property. The distance between the centre of gravity CG and the metacenter $M_{T}$ is called the metacentric height $\overline{G M}_{T}$. The shift of the $\mathrm{CB}$ will thus for small angles result in the restoring torque

$$
\rho \nabla \overline{G Z}(\phi) \approx \rho \nabla \overline{G M}_{T} \sin (\phi) \approx \rho \nabla \overline{G M}_{T} \phi
$$




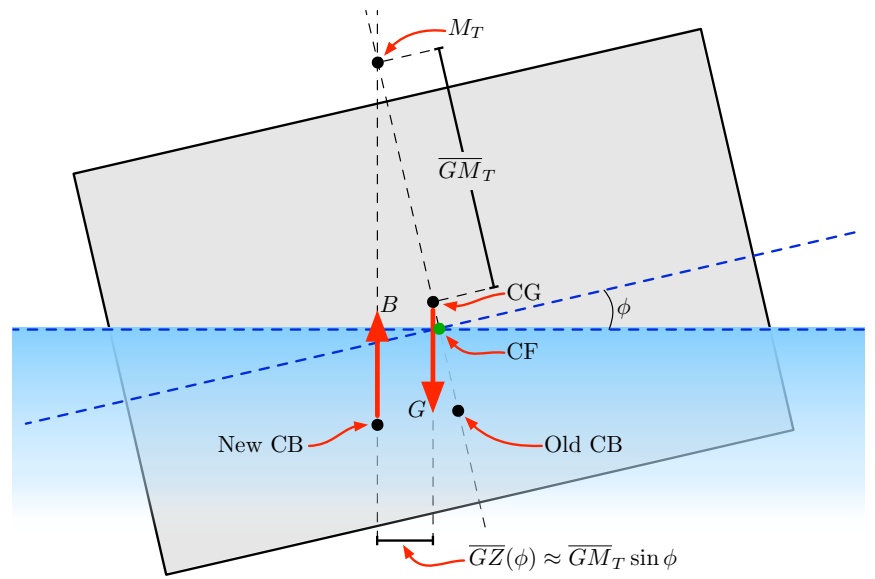

Figure 3.3: A boxed-shaped ship seen from the front. The ship has been perturbed from its equilibrium which shifts the $C B$ away from the original position. This shift creates a restoring torque. For small angles $\phi$, the righting arm is proportional to $\overline{G M}_{T} \sin (\phi)$.

In Figure 3.4 a sketch of a righting arm function is shown. Initially, the righting arm will increase as $\phi$ gets larger and then decrease until the righting arm becomes zero. If the roll angle $\phi$ increases after that point, the ship will capsize, see Lautrup (2004, p. 48-55) or Journée and Massie (2001) for a detailed discussion about ship stability. Note that the position of the metacenter $M_{T}$, the righting arm $\overline{G Z}(\phi)$ and the metacentric height $\overline{G M}_{T}$ may vary depending on a number of factors, such as hull shape and loading conditions.

In this thesis, the total restoring forces are assumed to be

$$
\boldsymbol{g}(\boldsymbol{\eta})=\left[0,0, \rho g C z, \rho \nabla \overline{G M}_{T} \phi, \rho \nabla \overline{G M}_{L} \theta, 0\right]^{T}
$$

where $\overline{G M}_{L}$ is the metacentric height in the pitch direction.

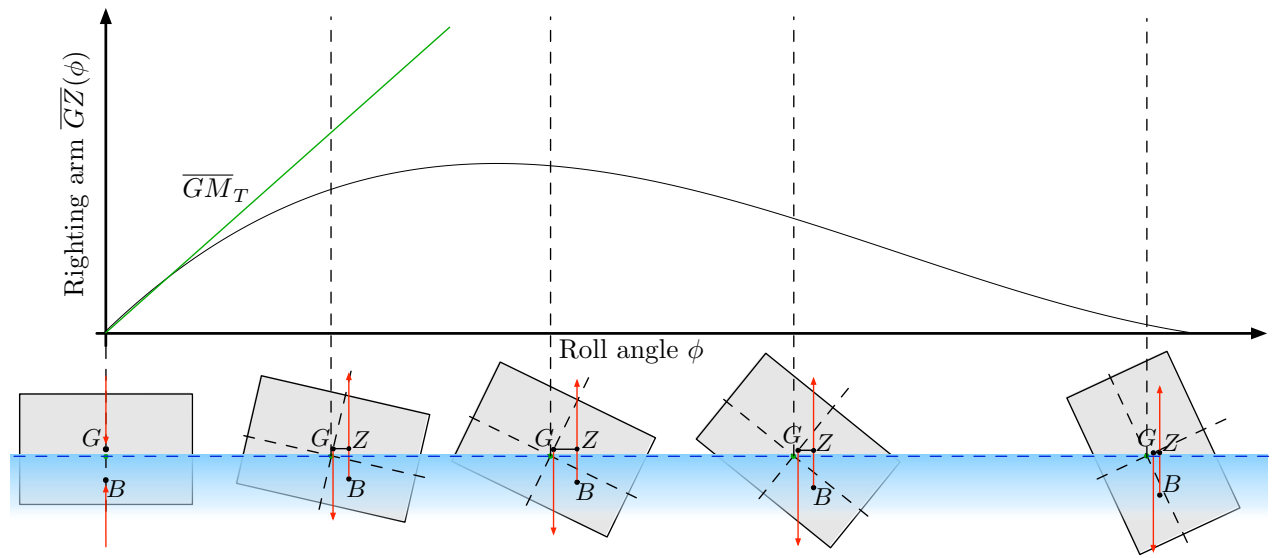

Figure 3.4: A sketch of a righting arm function as a function of the roll angle $\phi$. For small angles $\phi$, the righting arm is proportional to the roll angle, i.e. $\overline{G Z}(\phi) \approx \overline{G M}_{T} \phi$. As the roll angle $\phi$ increases further, the righting arm will eventually become zero which will result in the ship capsizing (Lautrup, 2004, p. $48-55)$. 


\subsubsection{Maneuvering Hydrodynamics}

Hydrodynamic forces and moments acting on a ship have two effects.

1. When a ship accelerates or decelerates in water, the water must separate around the ship and then join behind it. This change of momentum of the water can be modeled as a virtual added mass. As an example, consider that you try to move your arm through water. This will be more difficult than doing the same movement in air.

2. When a ship moves through water a certain damping, for instance, due to friction, will affect it.

The hydrodynamic forces and moments are modeled differently depending on the application. Here, the general nonlinear function $\boldsymbol{f}(\boldsymbol{\eta}, \boldsymbol{\nu}, \boldsymbol{\nu})$ is described using a series expansion. This expansion can, for instance, be split into terms depending on the derivatives of the body-fixed velocities (added mass), terms that are quadratic in the body-fixed velocities (added Coriolis-centripetal forces), terms depending linearly on the body-fixed velocities (potential/linear damping) and modulus terms (nonlinear damping). An example from Perez (2005, p. 83) of a model of hydrodynamic forces in roll is

$$
\begin{aligned}
& \tau_{p}=K_{\dot{v}} \dot{v}+K_{\dot{p}} \dot{p}+\ldots \quad \text { Added mass } \\
& +K_{\text {ur }} \text { ur Coriolis due to added mass } \\
& +K_{p} p+\ldots \quad \text { Linear damping } \\
& \left.\begin{array}{l}
+K_{|u| v}|u| v+K_{|v| v \mid}|v| v+K_{|r| v|r| v+\ldots} \mid \ldots \\
+K_{\phi|u v|} \phi|u v|+K_{\phi|u r|} \phi|u r|+\ldots
\end{array}\right\} \text { Nonlinear damping }
\end{aligned}
$$

where the coefficients are assumed to be constant. In general, it can be written on the form

$$
\boldsymbol{f}(\boldsymbol{\eta}, \dot{\nu}, \nu)=M_{A} \dot{\nu}+C_{A}(\nu) \nu+D(\eta, \nu)
$$

where the added mass inertia matrix

$$
\boldsymbol{M}_{A}=-\left[\begin{array}{cccccc}
X_{\dot{u}} & X_{\dot{v}} & X_{\dot{w}} & X_{\dot{p}} & X_{\dot{q}} & X_{\dot{r}} \\
Y_{\dot{u}} & Y_{\dot{v}} & Y_{\dot{w}} & Y_{\dot{p}} & Y_{\dot{q}} & Y_{\dot{r}} \\
Z_{\dot{u}} & Z_{\dot{v}} & Z_{\dot{w}} & Z_{\dot{p}} & Z_{\dot{q}} & Z_{\dot{r}} \\
K_{\dot{u}} & K_{\dot{v}} & K_{\dot{w}} & K_{\dot{p}} & K_{\dot{q}} & K_{\dot{r}} \\
M_{\dot{u}} & M_{\dot{v}} & M_{\dot{w}} & M_{\dot{p}} & M_{\dot{q}} & M_{\dot{r}} \\
N_{\dot{u}} & N_{\dot{v}} & N_{\dot{w}} & N_{\dot{p}} & N_{\dot{q}} & N_{\dot{r}}
\end{array}\right]
$$

and the added mass Coriolis matrix $\boldsymbol{C}_{A}(\boldsymbol{\nu})$ can be shown to have the same properties as the rigid-body equivalents (Ross, 2008).

A complete example will be given in Section 3.5. For a thorough treatment of hydrodynamic forces and moments for maneuvering models, see, for instance, Blanke and Christensen (1993), Perez (2005), Ross (2008) or Fossen (2011). 


\subsection{Actuators}

There are in principle two things affecting a ship, the environmental disturbances and the actuators, e.g. propellers, rudders or thrusters. These can be modeled with different levels of accuracy and complete models are very complex due to the interaction with the water, see for instance, Perez (2005) or Faltinsen (2005). In this section, simple linear models of a rudder and a propeller will be given. Note that most actuators found on a ship can be modeled as either a rotating propeller, i.e. with changing direction of thrust, or as a control surface acting like a fin.

\subsubsection{Rudder}

A rudder will create a rudder force $L$ and a drag force $D$ if the deflection angle is nonzero. These forces are in general dependent on, for instance, the forward speed $u$, the sway velocity $v$ and the yaw rate $r$. Here, we assume that the forces created by the rudder are proportional to the rudder angle $\delta$ which gives the model

$$
\boldsymbol{\tau}_{\mathrm{r}}=\left[\begin{array}{llllll}
-C_{1} & C_{2} & 0 & -z_{r} C_{2} & 0 & x_{r} C_{2}
\end{array}\right]^{T} \delta
$$

where $C_{1}, C_{2}>0$ are constants and $\boldsymbol{r}_{r}=\left[x_{r}, y_{r}, z_{r}\right]^{T}$ is the vector describing the rudder lever arm relative to the CG. A positive rudder angle $\delta$ is defined in the $b$ frame and is thus equivalent to a left turn. The $x$-component $x_{r}$ is more commonly known as the Lateral Center of Gravity (LCG) in ship literature.

\subsubsection{Propeller}

A propeller creates thrust by moving water in one direction. The main propeller of a ship mainly creates a negative thrust in the $x_{b}$-direction of the $b$-frame. Due to misalignment and since it is mounted below the CG, it will also create forces and moments in other directions. However, in this thesis, it is assumed that these effects are negligible and that the created force is proportional to the propeller $\mathrm{RPM} \omega_{p}$. The model is

$$
\boldsymbol{\tau}_{\mathrm{p}}=\left[\begin{array}{llllll}
C_{3} & 0 & 0 & 0 & 0 & 0
\end{array}\right]^{T} \omega_{p}
$$

\subsection{Environmental Disturbances}

When it comes to ships, there are three major environmental forces acting on the vessel, namely currents, wind and waves. The wave induced forces can be divided into first- and second-order effects. The first-order effects induce a zeromean oscillatory motion and the second-order effects are seen as a nonzero slowly varying drift.

The energy content of waves is described by using a power spectral density (PSD) function usually called a wave spectrum. Waves develop from wind that is forming small waves that grow until they break up. If the wind has been near stationary for a long time, the waves are considered to be fully developed (statistically stable) and the PSD contains a peak at lower frequencies. Because of changing wind 
strengths and directions, complex multi-modal directional wave spectra can be observed.

There are a number of common standard spectrum formulas that have been developed over a long time. Which family of spectra to use depend on the situation. They are usually dependent on one or two parameters that describe the sea-state. One example is the JONSWAP PSD (Fossen, 2011, p. 216) given by

$$
S(\omega)=0.2 H_{s}^{2} \omega_{0}^{4} \omega^{-5} \exp \left(-1.25 \omega_{0}^{4} \omega^{-4}\right) \gamma^{\exp \left(\frac{\omega_{0}-\omega}{\sqrt{2} \omega_{0} \sigma}\right)}
$$

where $H_{s}$ is the significant wave height, $\omega_{0}$ is the modal frequency and $\gamma$ is a constant that is usually chosen to be approximately 3.3. The parameter $\sigma$ is 0.07 if $\omega<\omega_{0}$ and $\sigma=0.09$ otherwise (Fossen, 2011, p. 206). An example of the JONSWAP PSD when $\gamma=3.3, H_{s}=1$ and $\omega_{0}=0.4$ can be seen in Figure 3.5.

For simulation purposes, the JONSWAP PSD can be approximated by letting white noise pass through a linear shaping filter, for instance,

$$
V(\mathrm{~s})=\frac{K_{0} \mathrm{~s}}{\mathrm{~s}^{2}+2 \zeta \omega_{a} \mathrm{~s}+\omega_{a}^{2}}
$$

where the parameters can be chosen such that the PSD $|V(\omega)|^{2}$ best represents the original spectrum (Fossen, 2011, p. 216).

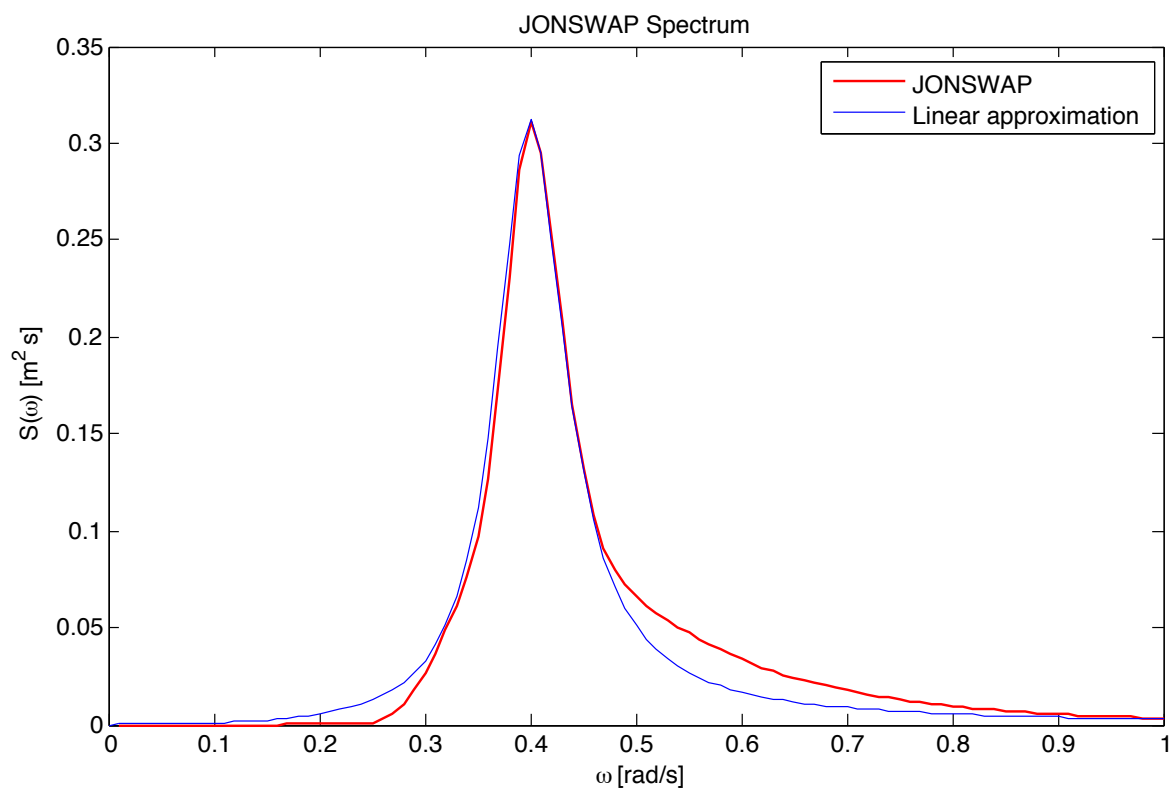

Figure 3.5: The red curve shows an example of a JONSWAP power spectral density function. The blue curve shows a linear approximation that can be used for simulation purposes. 


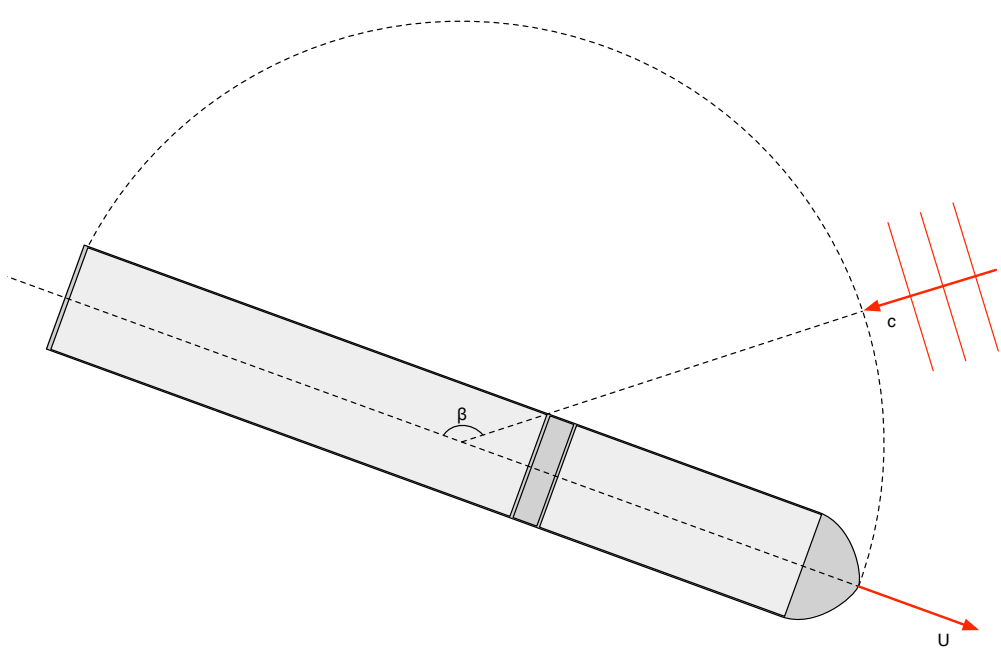

Figure 3.6: The perceived wave frequency, i.e. the encounter frequency $\omega_{e}$, is dependent on the ship's forward speed $U$, the actual wave frequency and the direction of the waves relative to the ship's heading. This is due to the Doppler effect.

Now, consider a ship moving with constant forward speed $U$ and constant heading. Since the ship is moving, the perceived wave frequency, called the encounter frequency, is dependent on the forward speed. The encounter frequency can be derived from Figure 3.6 where the relative speed between the ship and the waves is $c-U \cos (\beta)$ which gives the encounter frequency

$$
\omega_{e}=\left|\omega_{0}-\omega_{0}^{2} \frac{U}{g} \cos (\beta)\right|
$$

where $\omega_{0}$ is the wave frequency, $U$ is the forward speed, $\beta$ is the encounter angle, $g$ is the acceleration of gravity and the absolute value is used since a frequency can not be negative (Fossen, 2011, p. 210). The encounter angle is 0 when the waves are following the ship from behind and is increasing clockwise. It should be noted that since the perceived frequencies are different for a moving ship, this will not only change the frequency range but also the shape of the wave spectrum (Perez, 2005, p. 36). For more details, see for instance, Faltinsen (2005) or Fossen (2011).

The induced motions from waves are due to the varying pressure on the ship's hull and hence the hull geometry is needed to calculate the response given the wave spectrum and direction. Assuming a linear relation, the transfer functions from wave spectrum to forces on the ship are called force response amplitude operators (FRAOs).

The implications of this complex process disturbance is that the disturbance is colored, since the PSDs describing the energy content of the waves are narrowbanded or at least not flat, and that the peak frequency is dependent on ship pose and speed. 


\subsection{Surge-Sway-Roll-Yaw Maneuvering Model}

The approach used in this thesis concerns the roll motion. Hence, the surge-swayyaw maneuvering model extensively covered in the ship modeling literature is inadequate. Instead, in this section, a model similar to the four degree-of-freedom surge-sway-roll-yaw, maneuvering model with rudder input developed and discussed in Blanke and Christensen (1993) and Perez (2005) is presented. The difference is mainly in that the parameterization here is chosen to avoid identifiability issues.

With some abuse of notation, by neglecting the coupling to pitch and heave, the dynamic model defined in (3.6) and (3.10) to (3.13) can be written as

$$
\begin{aligned}
\dot{\boldsymbol{\eta}} & =\boldsymbol{J}(\boldsymbol{\eta}) \boldsymbol{\nu} \\
\boldsymbol{M}_{R B} \dot{\boldsymbol{\nu}}+C_{R B}(\boldsymbol{\nu}) \boldsymbol{\nu} & =\boldsymbol{\tau}
\end{aligned}
$$

where

$$
\begin{gathered}
\boldsymbol{J}(\boldsymbol{\eta})=\left[\begin{array}{cccc}
c_{\psi} & -c_{\phi} s_{\psi} & 0 & 0 \\
s_{\psi} & c_{\phi} c_{\psi} & 0 & 0 \\
0 & 0 & 1 & 0 \\
0 & 0 & 0 & c_{\phi}
\end{array}\right], \\
\boldsymbol{M}_{R B}=\left[\begin{array}{cccc}
\bar{M} & 0 & 0 & -\bar{M} \bar{y}_{g} \\
0 & \bar{M} & -\bar{M} \bar{z}_{g} & \bar{M} \bar{x}_{g} \\
0 & -\bar{M} \bar{z}_{g} & \bar{I}_{x} & 0 \\
-\bar{M} \bar{y}_{g} & \bar{M} \bar{x}_{g} & 0 & \bar{I}_{z}
\end{array}\right], \\
\boldsymbol{C}_{R B}(\boldsymbol{\nu})=\left[\begin{array}{cccc}
0 & -\bar{M} r & \bar{M} \bar{z}_{g} r & -\bar{M} \bar{x}_{g} r \\
\bar{M} r & 0 & -\bar{M} \bar{y}_{g} p & -\bar{M} \bar{y}_{g} r \\
-\bar{M} \bar{z}_{g} r & \bar{M} \bar{y}_{g} p & 0 & 0 \\
\bar{M} \bar{x}_{g} r & \bar{M} \bar{y}_{g} r & 0 & 0
\end{array}\right],
\end{gathered}
$$

$\boldsymbol{\eta}=[x, y, \phi, \psi]^{T}$ and $\boldsymbol{\nu}=[u, v, p, r]^{T}$. Note that the $3 \times 4$ and $4 \times 3$ elements of $\boldsymbol{C}_{R B}(\boldsymbol{\nu})$ are 0 due to the to the chosen representation. The ship's total mass is assumed to be $\bar{M}$ located at $\boldsymbol{r}_{g}=\overline{\boldsymbol{r}}_{g}=\left[\bar{x}_{g}, \bar{y}_{g}, \bar{z}_{g}\right]^{T}$ and $\bar{I}_{x}$ and $\bar{I}_{z}$ are the inertias expressed in the $b$-frame. The forces acting on the ship are described in the earlier sections of this chapter. These forces are

$$
\boldsymbol{\tau}=\boldsymbol{\tau}_{\text {hydro }}+\boldsymbol{\tau}_{\text {actuators }}+\boldsymbol{\tau}_{\text {environmental }}
$$

Firstly, the hydrodynamic forces and moments acting on the ship are described using the theory presented in Section 3.2, i.e.

$$
\tau_{\text {hydro }}=-M_{A} \dot{\nu}+\bar{f}(\boldsymbol{\eta}, \nu)-\boldsymbol{g}(\boldsymbol{\eta})
$$

where $\overline{\boldsymbol{f}}(\boldsymbol{\eta}, \boldsymbol{\nu})$ is the Coriolis terms combined with the nonlinear damping and $\boldsymbol{M}_{A}$ is the added mass matrix. The added mass matrix is assumed to be

$$
M_{A}=-\left[\begin{array}{cccc}
X_{\dot{u}} & 0 & 0 & 0 \\
0 & Y_{\dot{v}} & Y_{\dot{p}} & Y_{\dot{r}} \\
0 & K_{\dot{v}} & K_{\dot{p}} & K_{\dot{r}} \\
0 & N_{\dot{v}} & N_{\dot{p}} & N_{\dot{r}}
\end{array}\right]
$$


which is defined in (3.23) but where the coupling to surge has been neglected due to the assumption that the surge motion has much slower dynamics. The nonlinear hydrodynamic damping and Coriolis affects are assumed to be described by

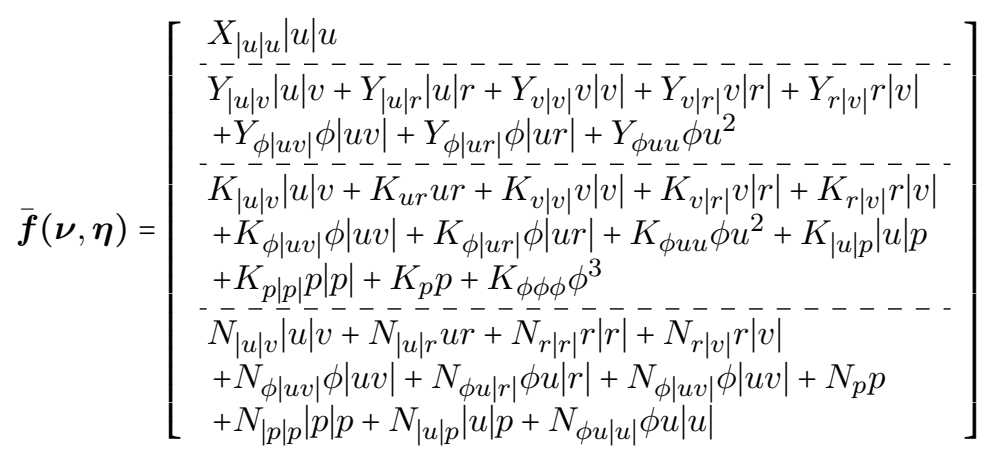

where the coefficients are assumed to be constant (Perez, 2005, p. 83). Assuming that the roll angle $\phi$ is small, the hydrostatic forces and moments are given by

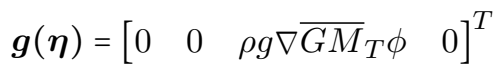

defined in (3.20).

Secondly, the only actuator acting on the ship is assumed to be the simplified rudder described in Section 3.3.1 and a constant force acting as the forward propulsion. The actuator contribution is

$$
\boldsymbol{\tau}_{\text {actuators }}=\left[\begin{array}{llll}
C_{3} \omega_{p}-C_{1} \delta & C_{2} \delta & -z_{r} C_{2} \delta & x_{r} C_{2} \delta
\end{array}\right]^{T}=\boldsymbol{R}(\delta)
$$

Finally, the environmental forces acting on the ship are assumed to have the properties described in Section 3.4 but are otherwise unknown.

The total model is

$$
\begin{aligned}
\dot{\boldsymbol{\eta}} & =\boldsymbol{J}(\boldsymbol{\eta}) \boldsymbol{\nu} \\
\boldsymbol{M} \dot{\boldsymbol{\nu}} & =-\boldsymbol{C}_{R B}(\boldsymbol{\nu}) \boldsymbol{\nu}+\overline{\boldsymbol{f}}(\boldsymbol{\nu}, \boldsymbol{\eta})-\boldsymbol{g}(\boldsymbol{\eta})+\boldsymbol{R}(\delta)+\boldsymbol{\tau}_{\text {environmental }}
\end{aligned}
$$

where the inertia matrix is

$$
\boldsymbol{M}=\boldsymbol{M}_{R B}+\boldsymbol{M}_{A}=\left[\begin{array}{cccc}
\bar{M}-X_{\dot{u}} & 0 & 0 & -\bar{M} \bar{y}_{g} \\
0 & \bar{M}-Y_{\dot{v}} & -\bar{M} \bar{z}_{g}-Y_{\dot{p}} & \bar{M} \bar{x}_{g}-Y_{\dot{r}} \\
0 & -\bar{M} \bar{z}_{g}-K_{\dot{v}} & \bar{A}_{x} & -K_{\dot{r}} \\
-\bar{M} \bar{y}_{g} & \bar{M} \bar{x}_{g}-N_{\dot{v}} & -N_{\dot{p}} & \bar{A}_{z}
\end{array}\right]
$$

Here, the parameters $\bar{A}_{x}=\bar{I}_{x}-K_{\dot{p}}$ and $\bar{A}_{z}=\bar{I}_{z}-N_{\dot{r}}$ have been introduced to avoid identifiability issues. 


\subsubsection{Non-minimum Phase Behavior}

A surface vessel will generally show a non-minimum phase behavior in the response from the rudder angle $\delta$ to the roll angle $\phi$. This can be explained by the location of the rudder and the coupling between the different degrees of freedom. Figure 3.7(a) shows the rudder location in relation to the $\mathrm{CF}$ and the $\mathrm{CG}$. When a positive rudder angle $\delta$ is applied the ship will initially lean inwards (left). This is due to the rudder's location below the CG and that the roll dynamics is faster than the other subsystems, see Figure 3.7(b). Eventually, coupling from the slower dynamics, especially the yaw subsystem, and hydrodynamic forces will create a torque in the opposite direction resulting in the ship leaning outwards, see Figure 3.7(c) (Perez, 2005, p. 150).

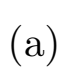

(a)
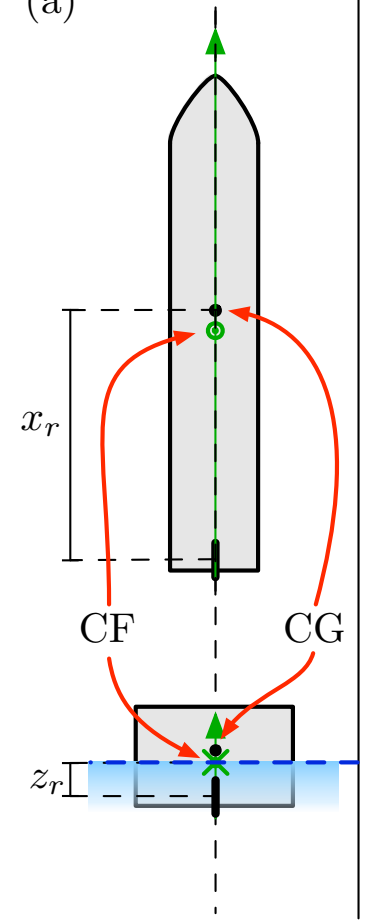

(b)

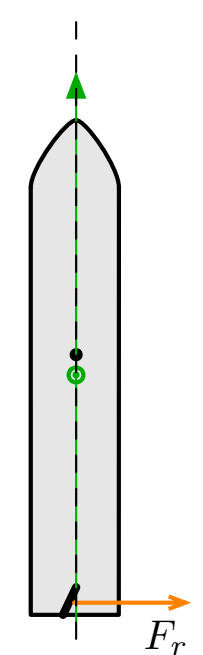

11

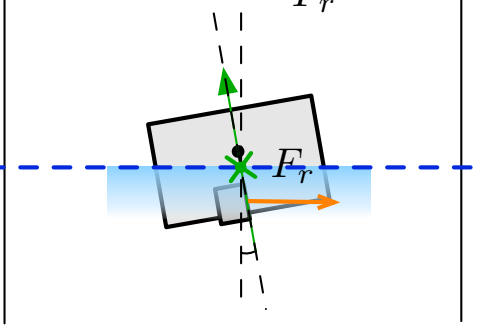

(c)

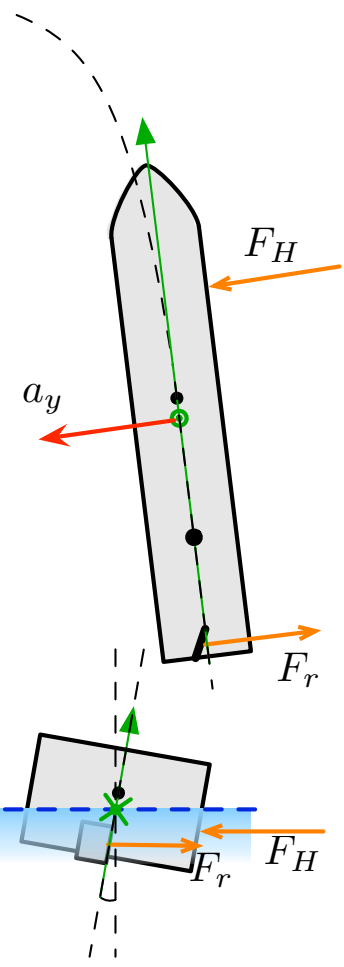

Figure 3.7: The non-minimum phase behavior from the rudder angle $\delta$ to the roll angle $\phi$ can be explained with interaction between the subsystems. Here, $F_{r}$ is the force generated by the rudder and $F_{H}$ corresponds to the hydrodynamic forces. 


\subsection{Sensors}

In this section, the inertial measurement unit (IMU) and its measurement equations, which are used extensively through out the thesis, will be described.

\subsubsection{Inertial Measurement Unit}

In this thesis, the ship motion is assumed to be observed with a three degrees-offreedom strap-down inertial measurement unit (IMU) and a schematic view of the IMU's position can be seen in Figure 3.8. Firstly, the IMU measures the angular velocity

$$
y_{1, t}=\dot{\phi}_{t}+e_{1, t}
$$

around the $x_{s}$-axis in the $s$-frame, at discrete times $t$, where $\dot{\phi}_{t}$ is the sampled system's angular velocity and $e_{1, t}$ is discrete-time white zero-mean measurement noise.

Secondly, the observed tangential acceleration at any point a fixed distance $D$ from the CR due to the rotation around the $x_{s}$-axis in the $s$-frame is

$$
a=D \ddot{\phi}
$$

Given that the roll angle $\phi$ is small, the tangential acceleration sensed by the IMU is thus

$$
a_{s}=z_{s} \ddot{\phi}+g \phi-a_{y},
$$

where the first term is the contribution from the angular acceleration, the second term is due to gravity and the third term is the acceleration of the CR in the $y_{n}$-axis in the $n$-frame. The parameter $-z_{s}$ is the distance from the CR to the origin of the IMU coordinate system.

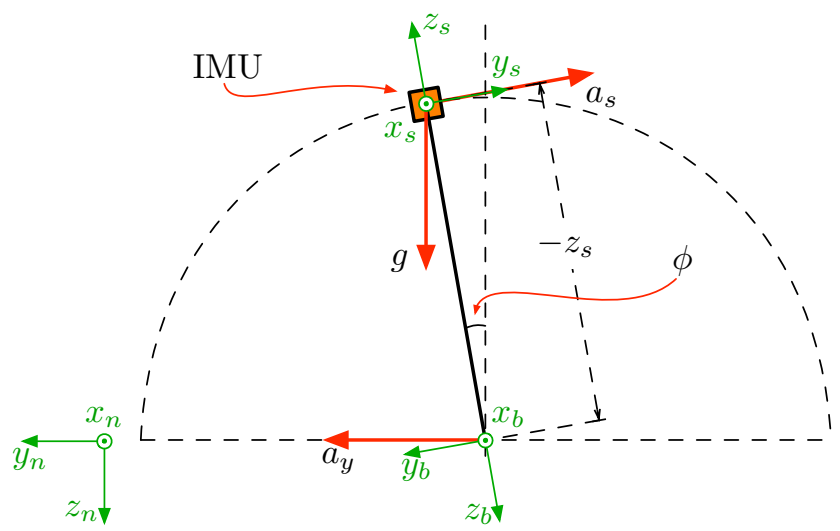

Figure 3.8: The 3DOF IMU is assumed to measure the tangential acceleration $a_{s}$, the angular velocity around the $x_{s}$-axis and the angular velocity around the $z_{s}$-axis. Note that the IMU's $s$-frame has a different orientation than the $b$-frame. 
The IMU measures this tangential acceleration

$$
y_{2, t}=a_{s, t}+e_{2, t}
$$

at discrete times $t$, where the first term is (3.43) after sampling and the second term is discrete-time white zero-mean measurement noise.

Finally, the IMU measures the angular velocity

$$
y_{3, t}=-r_{t}+e_{3, t}
$$

around the $z_{s}$-axis in the $s$-frame, at discrete times $t$, where $r_{t}$ is the sampled system's angular velocity and $e_{3, t}$ is discrete-time white zero-mean measurement noise.

The IMU that has been used in the experiments can be seen in Figure 3.9. It is an Xsens MTi-G, equipped with a three-axis accelerometer, a three-axis gyro, a threeaxis magnetometer and a GPS unit. Only a few of the axes will be used to collect data. The $y$-axis of the accelerometer corresponds to the tangential acceleration (3.44), the $x$-axis of the gyro corresponds to the angular velocity (3.41) while the $z$-axis of the gyro corresponds to the angular velocity (3.45).

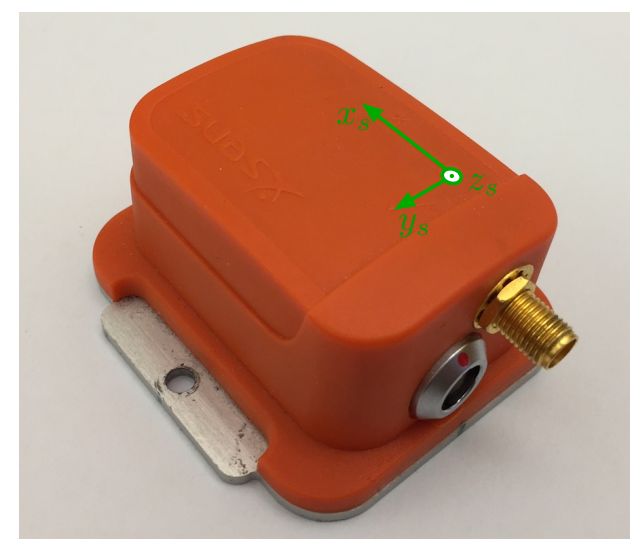

Figure 3.9: A Xsens MTi-G. The silver socket is the data-connection and the golden socket is the connection for the GPS antenna. Note the coordinate system (enhanced in software) on top of the IMU with the $x$-axis pointing inwards and left, the $y$-axis pointing downwards and left, and the $z$-axis pointing upwards. 


\section{Graybox Modelling of a Second-order Subsystem}

This chapter presents a study on the estimation of the physical parameters in a graybox model of an inverted pendulum. Estimation of physical parameters in a mechanical system is notoriously difficult if the input to the system is unknown. This is partly due to identifiability issues, i.e. several combinations of inputs and physical parameters will all satisfy the equations of motion. In this work, it is assumed that the true input to the system is unknown and that only measurements of the motion are available, together with a signal related to the true input. The unknown input issues are resolved by eliminating the true input from the model and instead using an acceleration measurement as the input to the identification procedure. The resulting model is of second order and despite its simplicity, this approach can be used for estimation of physical parameters in a multitude of vehicles, such as, cars, trucks, trains and ships. The model derived in this chapter will be further discussed in Chapter 5 where it will be shown to be a subsystem of the complete dynamics of a ship.

\subsection{The Force-Mass Ambiguity}

Mass is one of the most influential parameters in most mechanical systems. However, if only motion data are known, it is difficult to uniquely determine the system's mass in many cases. A central issue when only measuring the motion of a system is the force-mass ambiguity. For instance, consider the right figure of Figure 4.1, in which the acceleration $a$ of a box with mass $m$, affected by the

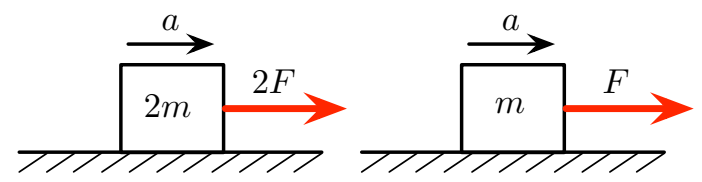

Figure 4.1: This is an example of the force-mass ambiguity. If only the acceleration is measured, the left and right systems are indistinguishable. 
force $F$ and moving in one dimension is directly proportional to $F / m$. If only $a$ is measured, there are still multiple combinations of $F$ and $m$ that will satisfy the equation as, for instance, shown in Figure 4.1. This ambiguity can be overcome with special experiments where the force acting on the system is known, or by measuring the force acting on the system. However, doing special experiments is in many cases intractable or too expensive. Instead, the approach considered in this thesis is to estimate the change in mass by using two datasets where the mass is known in the first dataset and unknown in the second dataset. The first dataset will be called the nominal dataset since it corresponds to a nominal loading case. The introduction of this extra information, i.e. the nominal dataset, is sufficient to overcome the ambiguity. The advantage of this method is that once the nominal case is known, it can be used as a basis for comparison for data collected during normal operation. This makes it possible to estimate the current mass at any given time, assuming that enough data has been collected.

\subsection{Approximate Model of a Pendulum}

Consider the system in Figure 4.2, which is an inverted pendulum hinged on a cart. The pendulum's mass is $M$ with its center of gravity a distance $z_{g}$ from the center of rotation $(\mathrm{CR})$ and with the inertia $I_{x}$ around its center of gravity. Furthermore, a mass $m$ with inertia $I_{x, m}$ is introduced a distance $z_{m}$ from the CR.

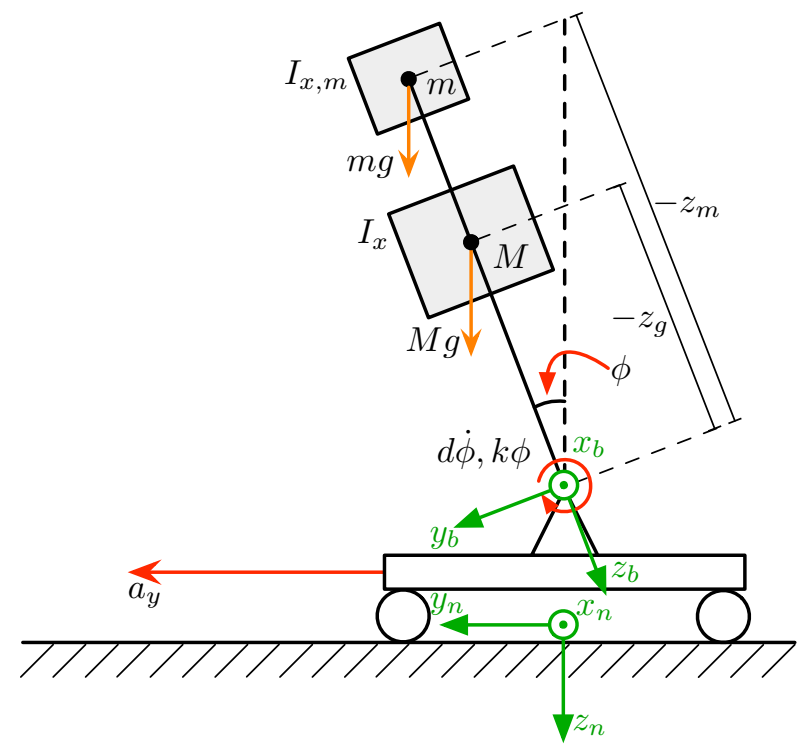

Figure 4.2: $A$ sketch relating the important quantities of the approximate inverted pendulum model. Rigidly attached to the pendulum is the NED body-fixed coordinate system (in green) located at the center of rotation. There is also a world-fixed coordinate system and the angle $\phi$ is the relative rotation of the $x_{b}$-axis around the $x_{n}$-axis. The orange arrows correspond to forces acting on the pendulum while the red straight arrow corresponds to the unknown acceleration $a_{y}$. Note that the $C R$ coincides with the $x_{b}$-axis. 


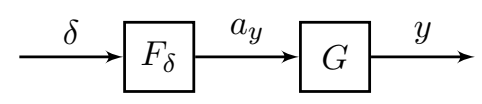

Figure 4.3: The acceleration $a_{y}$ is unknown but is correlated with the known signal $\delta$. The signals $a_{y}$ and $\delta$ are assumed to be related through the unknown subsystem $F_{\delta}(p)$.

There are two torsional torques acting on the pendulum, one linearly dependent on the angle $\phi$ corresponding to the restoring forces, and one linearly dependent on the angular velocity $\dot{\phi}$ corresponding to damping, for instance due to friction.

The cart is disturbed by an external force that results in the acceleration $a_{y}$ which will be considered to be unknown. Here we will focus on the modelling of the pendulum. However, the pendulum is assumed to be a subsystem of a larger system and the acceleration $a_{y}$ is assumed to be related with a known signal $\delta$ through some unknown system $F_{\delta}(\mathrm{p})$, see Figure 4.3 . As an example, in Chapter 5 the pendulum will correspond to the roll dynamics, the larger system is the ship itself and $\delta$ is the rudder angle.

There is a torque disturbance $\bar{\tau}$ acting on the center of rotation. The torque disturbance $\bar{\tau}$ is not assumed to have any specific distribution. The disturbance is an aggregate of neglected couplings to other subsystems and other types of disturbances. For the ship in Chapter 5, examples of disturbances are vibrations from the engine and environmental disturbances such as waves or wind.

The dynamic model can be derived, for instance, by using Newton's second law. For a linear motion this relates the mass $m$, the acceleration $a$ and the forces $F_{i}$ acting on the system according to

$$
\sum_{i} F_{i}=m a
$$

Similarly, for a rotational motion the law relates the inertia $I$, the angular velocity $\omega$ and the torques $\tau_{i}$ acting on the system according to

$$
\sum_{i} \tau_{i}=I \dot{\omega}
$$

Consider Figure 4.4 where the cart-pendulum system in Figure 4.2 has been split into the cart and the pendulum, the forces acting on each part have been marked with orange arrows and the accelerations with red straight arrows. The forces $N$ and $R_{y}$ are reaction forces and $f_{y}$ is the force acting on the pendulum cart. The two masses are lumped into one new point mass $\bar{M}$, at the position $\bar{z}_{g}$ with the inertia $\bar{I}_{x}$. These lumped parameters are given by basic mechanics and are defined as

$$
\bar{M}=M+m, \quad \bar{z}_{g}=\frac{M z_{g}+m z_{m}}{M+m}
$$

and

$$
\bar{I}_{x}=I_{x}+I_{x, m}+M\left(\bar{z}_{g}-z_{g}\right)^{2}+m\left(\bar{z}_{g}-z_{m}\right)^{2}
$$




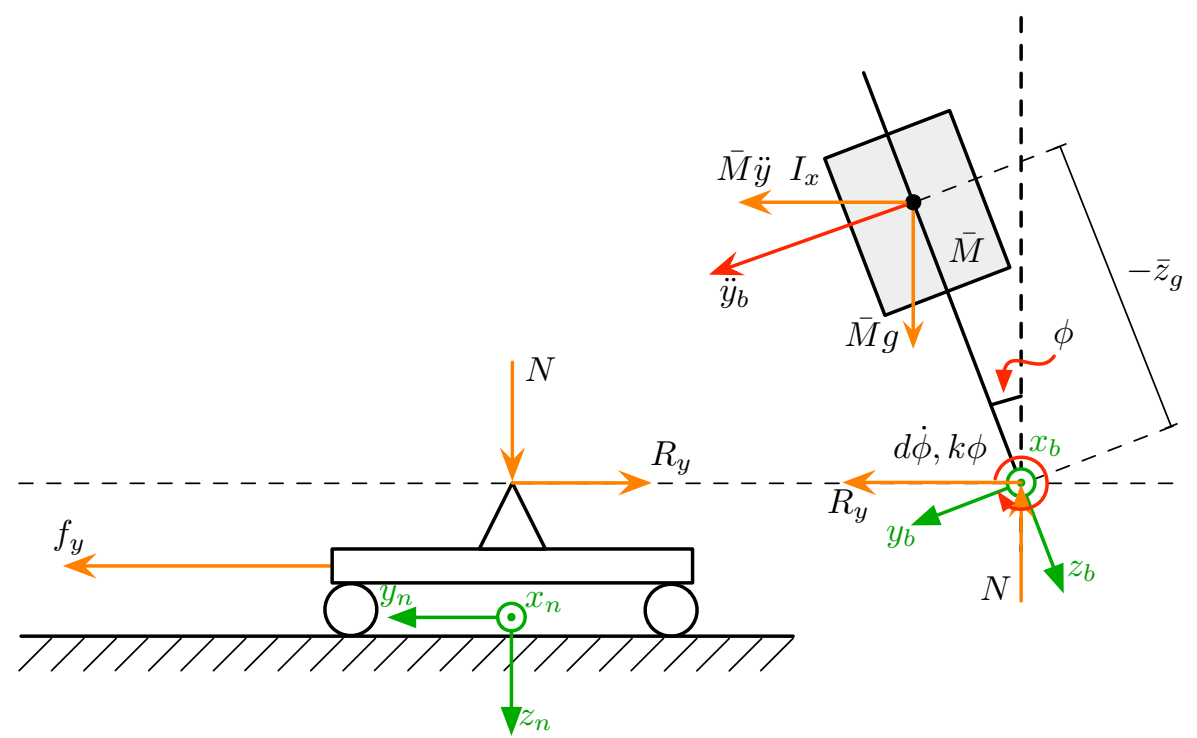

Figure 4.4: A sketch relating the important quantities of the approximate inverted pendulum model.

Firstly, note that the tangential acceleration at the lumped center of gravity is

$$
\ddot{y}_{b}=\ddot{y} \cos (\phi)-\bar{z}_{g} \ddot{\phi}
$$

where $\ddot{y}$ is the acceleration in the world-fixed $y_{n}$-axis and $\ddot{\phi}$ is the angular acceleration around the body-fixed $x_{b}$-axis. Hence, the force equilibrium in the lumped center of gravity in the (pendulum) body-fixed $y_{b}$-axis is given by

$$
\bar{M}\left(\ddot{y} \cos (\phi)-\bar{z}_{g} \ddot{\phi}\right)=-N \sin (\phi)+R_{y} \cos (\phi)+\bar{M} g \sin (\phi)
$$

and moment equilibrium around the lumped center of gravity gives

$$
\bar{I}_{x} \ddot{\phi}=-\bar{z}_{g}\left(N \sin (\phi)-R_{y} \cos (\phi)\right)-d \dot{\phi}-k \phi+\bar{\tau}
$$

By combining (4.5) with (4.6) and introducing $a_{y}=\ddot{y}$, the resulting dynamic model can be written as the differential equation

$$
\left(\bar{I}_{x}+\bar{M} \bar{z}_{g}^{2}\right) \ddot{\phi}=-k \phi-\bar{M} \bar{z}_{g} g \sin (\phi)-d \dot{\phi}+\bar{M}_{\bar{z}} \cos (\phi) a_{y}+\bar{\tau}
$$

which is a mass-spring-damper model. Now note that

$$
\bar{M} \bar{z}_{g} \stackrel{(4.3)}{=} M z_{g}+m z_{m} \quad \text { and } \quad \bar{I}_{x}+\bar{M} \bar{z}_{g}^{2} \stackrel{(4.3)}{=} I_{x}+M z_{g}^{2}+I_{x, m}+m z_{m}^{2}
$$

which gives

$$
I_{1} \ddot{\phi}=-k \phi-\left(M z_{g}+m z_{m}\right) g \sin (\phi)-d \dot{\phi}+\left(M z_{g}+m z_{m}\right) \cos (\phi) a_{y}+\bar{\tau}
$$

where $I_{1}=I_{x}+M z_{g}^{2}+I_{x, m}+m z_{m}^{2}$. For small angles $\phi$, i.e. linearizing around zero, (4.9) can be simplified to

$$
I_{1} \ddot{\phi}=\overbrace{-\left(k+M g z_{g}+m g z_{m}\right) \phi}^{\text {restoring torque }} \overbrace{-d \dot{\phi}}^{\text {damping }} \overbrace{+\left(M z_{g}+m z_{m}\right) a_{y}+\bar{\tau}}^{\text {disturbances }}
$$


For brevity, the time-dependency of the states and disturbances has been dropped from the expression.

In the same way, force equilibrium in the world-fixed $y_{n}$-axis gives

$$
\bar{M} \ddot{y}=f_{y}-\bar{M} \bar{z}_{g} \dot{\phi}^{2} \sin (\phi)+\bar{M} \bar{z}_{g} \ddot{\phi} \cos (\phi)
$$

which by inserting (4.3) is equivalent to

$$
(M+m) \ddot{y}=f_{y}-\left(M z_{g}+m z_{m}\right) \dot{\phi}^{2} \sin (\phi)+\left(M z_{g}+m z_{m}\right) \ddot{\phi} \cos (\phi)
$$

but since the model has the acceleration as input, the force generating the acceleration is of less importance and hence, (4.12) will not be used.

The motion of the pendulum is assumed to be measured by the IMU described in Section 3.6.1. The measurement equations are recapitulated here for readability. The IMU measures the angular velocity around the $x_{b}$-axis

$$
y_{1, t}=\dot{\phi}_{t}+e_{1, t}
$$

defined in (3.41) and the tangential acceleration

$$
y_{2, t}=a_{s, t}+e_{2, t}, \quad a_{s}=z_{s} \ddot{\phi}+g \sin (\phi)-a_{y} \cos (\phi) \underset{\tilde{\phi}}{\operatorname{small}} z_{s} \ddot{\phi}+g \phi-a_{y}
$$

defined in (3.44). Note that the IMU is assumed to have a NWU coordinate system while the body-fixed coordinate system is NED, see Figure 4.5.

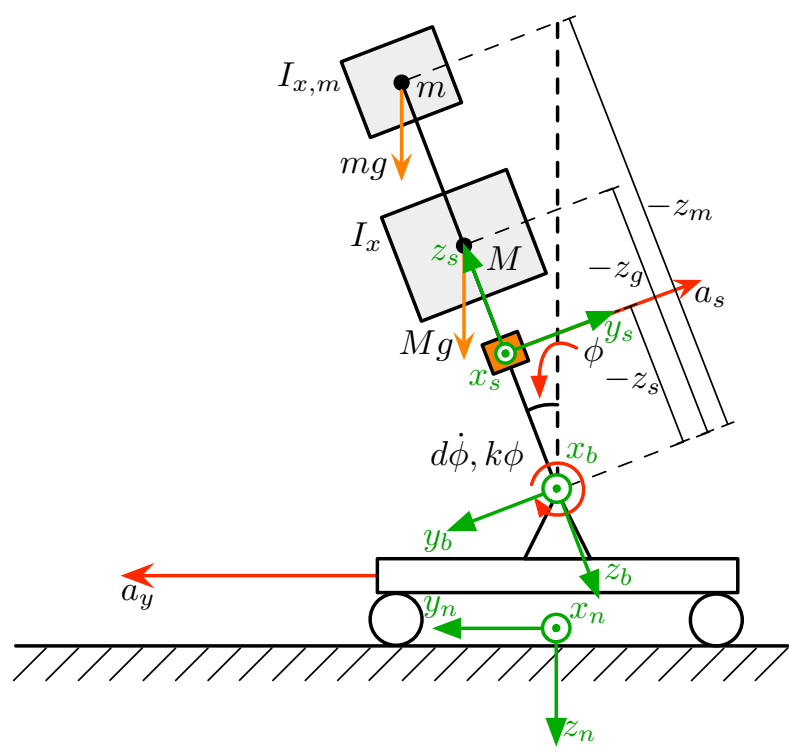

Figure 4.5: A sketch showing the coordinate system of the IMU in relation to the (pendulum) body-fixed coordinate system. Note that the body-fixed system is NED while the sensor system is NWU. 


\subsubsection{Identifiability of the Approximate Model}

Uniqueness is a key aspect for models containing a large number of parameters. For a model parameterized with parameters corresponding to physical quantities this is especially important since a non-unique description will imply that the parameters can assume several different value and still satisfy the dynamic model.

To investigate the uniqueness of the model (4.10), an identifiability analysis will be performed. The goal of the analysis is to understand what is possible to estimate given that the true input $a_{y}$ is known. The structural identifiability method described in Section 2.5 will be used and the analysis is performed using symbolic software. It should be stressed that this method can be used to investigate local identifiability; for global methods, see for instance, Ljung and Glad (1994).

The model (4.10), with the output defined by (3.41) and (3.44), can be cast into a state-space form by choosing the states $x=[\phi, \dot{\phi}]^{T}$ and inserting (4.10) into (3.44). The effects of measurement and process noise are neglected in this section, i.e. $e_{1}=e_{2}=\bar{\tau}=0$. The resulting state-space model is given by

$$
\begin{aligned}
& \dot{x}=\left[\begin{array}{cc}
0 & 1 \\
-\frac{k+M g z_{g}+m g z_{m}}{I_{1}} & -\frac{d}{I_{1}}
\end{array}\right] x+\left[\begin{array}{c}
0 \\
\frac{M z_{g}+m z_{m}}{I_{1}}
\end{array}\right] u=f(x, u, \vartheta) \\
& y=\left[\begin{array}{cc}
0 & 1 \\
-\frac{k+M g z_{g}+m g z_{m}}{\tilde{I}_{1}}+g & -\frac{d}{\tilde{I}_{1}}
\end{array}\right] x+\left[\begin{array}{c}
0 \\
\frac{M z_{g}+m z_{m}-\tilde{I}_{1}}{\tilde{I}_{1}}
\end{array}\right] u=h(x, u, \vartheta)
\end{aligned}
$$

where $I_{1}$ is defined in Section $4.2, \tilde{I}_{1}=I_{1} / z_{s}$ and $u=a_{y}$. Note that $f(x, u, \vartheta)$ and $h(x, u, \vartheta)$ symbolize the extended state-space model (2.39). The parameters considered unknown are

$$
\vartheta_{p}=\left[\begin{array}{llllllll}
M & z_{g} & k & I_{x} & d & I_{x, m} & m & z_{m}
\end{array}\right]^{T}
$$

The remaining parameters are assumed to be known. There are $n_{x}=2$ states and $n_{\vartheta}=8$ parameters, i.e. the extended nonlinear system has dimension 10. All parameters are assumed to be non-zero and distinctly different from each other, unless otherwise stated, e.g. $z_{g}$ and $z_{m}$ are never treated as equal even if they might assume the same numerical value.

The complete extended observability matrix $\mathcal{O}$ for the general case is unfortunately not possible to recite due to its size and complexity. Instead, an illustrative special case will be presented in detail to show the typical structure and then, the extended observability matrix $\mathcal{O}$ for the general case is presented in row-reduced form together with conclusions.

\section{Illustrative Example - A Special Case}

This section presents an example that will show identifiability of the parameters $I_{x}$, $k$ and $d$ in the model (4.13) when there is no additional mass, i.e. $I_{x, m}=m=z_{m}=0$. The intention is to get a feeling for the structure of the extended observability matrix $\mathcal{O}$. In this example, only the first measurement is used, the output is $y=\dot{\phi}$ and the input $u=a_{y}$, which for the analysis is assumed to be known. For notational simplicity, the parameters $z_{g}$ and $g$ are assumed to be equal to 1 . Since 
there are five states in the extended state-space model, i.e. two states and three parameters, at most four time derivatives of the output have to be considered. In this case, the extended observability matrix is

$$
\mathcal{O}=\left[\begin{array}{c:c:c:c:c}
\frac{\partial h(x, u, \vartheta)}{\partial x_{1}} & \frac{\partial h(x, u, \vartheta)}{\partial x_{2}} & \frac{\partial h(x, u, \vartheta)}{\partial I_{x}} & \frac{\partial h(x, u, \vartheta)}{\partial k} & \frac{\partial h(x, u, \vartheta)}{\partial d} \\
\vdots & \vdots & \vdots & \vdots & \vdots \\
\underbrace{\frac{\partial h^{(4)}(x, u, \vartheta)}{\partial x_{1}}}_{\mathcal{O}_{x_{1}}} & \underbrace{\frac{\partial h^{(4)}(x, u, \vartheta)}{\partial x_{2}}}_{\mathcal{O}_{x_{2}}} & \underbrace{\frac{\partial h^{(4)}(x, u, \vartheta)}{\partial I_{x}}}_{\mathcal{O}_{I_{x}}} & \underbrace{\frac{\partial h^{(4)}(x, u, \vartheta)}{\partial k}}_{\mathcal{O}_{k}} & \underbrace{\frac{\partial h^{(4)}(x, u, \vartheta)}{\partial d}}_{\mathcal{O}_{d}}
\end{array}\right]
$$

and the corresponding columns are

$$
\begin{aligned}
& \mathcal{O}_{x_{1}}=\left[\begin{array}{c}
0 \\
-\frac{M_{k}}{I_{1}} \\
\frac{d M_{k}}{I_{1}^{2}} \\
\frac{\left(I_{k}-d^{2}\right) M_{k}}{I_{1}^{3}} \\
\frac{d\left(d^{2}-2 I_{k}\right) M_{k}}{I_{1}^{4}}
\end{array}\right], \quad \mathcal{O}_{x_{2}}=\left[\begin{array}{c}
1 \\
-\frac{d}{I_{1}} \\
\frac{d^{2}-I_{k}}{I_{1}^{2}} \\
\frac{d\left(2 I_{k}-d^{2}\right)}{I_{1}^{3}} \\
\frac{d^{4}-\left(3 d^{2}-I_{k}\right) I_{k}}{I_{1}^{4}}
\end{array}\right], \\
& \mathcal{O}_{k}=\left[\begin{array}{c}
0 \\
-\frac{1}{I_{1}} x_{1} \\
\frac{d}{I_{1}^{2}} x_{1}-\frac{1}{I_{1}} x_{2} \\
\frac{2 I_{k}-d^{2}}{I_{1}^{3}} x_{1}+\frac{2 d}{I_{1}^{2}} x_{2}+U_{k}(0, u) \\
\frac{d\left(d^{2}-4 I_{k}\right)}{I_{1}^{4}} x_{1}+\frac{2 I_{k}-3 d^{2}}{I_{1}^{3}} x_{2}+U_{k}(u, \dot{u})
\end{array}\right] \\
& \mathcal{O}_{d}=\left[\begin{array}{c}
0 \\
-\frac{1}{I_{1}} x_{2} \\
\frac{M_{k}}{I_{1}^{2}} x_{1}+\frac{2 d}{I_{1}^{2}} x_{2}+U_{d}(0,0, u) \\
-\frac{2 d M_{k}}{I_{1}^{3}} x_{1}+\frac{2 I_{k}-3 d^{2}}{I_{1}^{3}} x_{2}+U_{d}(0, u, \dot{u}) \\
\frac{\left(3 d^{2}-2 I_{k}\right) M_{k}}{I_{1}^{4}} x_{1}+\frac{d\left(4 d^{2}-6 I_{k}\right)}{I_{1}^{4}} x_{2}+U_{d}(u, \dot{u}, \ddot{u})
\end{array}\right], \\
& \mathcal{O}_{I_{x}}=\left[\begin{array}{c}
0 \\
\frac{M_{k}}{I_{1}^{2}} x_{1}+\frac{d}{I_{1}^{2}} x_{2}+U_{I}(0,0,0, u) \\
-\frac{2 d M_{k}}{I_{1}^{3}} x_{1}+\frac{I_{k}-2 d^{2}}{I_{1}^{3}} x_{2}+U_{I}(0,0, u, \dot{u}) \\
\frac{\left(3 d^{2}-2 I_{k}\right) M_{k}}{I_{1}^{4}} x_{1}+\frac{d\left(3 d^{2}-4 I_{k}\right)}{I_{1}^{4}} x_{2}+U_{I}(0, u, \dot{u}, \ddot{u}) \\
\frac{2 d\left(3 I_{k}-2 d^{2}\right) M_{k}}{I_{1}^{5}} x_{1}-\frac{\left(d^{2}-2 I_{k}\right)\left(4 d^{2}-I_{k}\right)}{I_{1}^{5}} x_{2}+U_{I}\left(u, \dot{u}, \ddot{u}, u^{(3)}\right)
\end{array}\right], \\
& U_{k}\left(u_{1}, u_{2}\right)=\frac{2 d M}{I_{1}^{3}} u_{1}-\frac{M}{I_{1}^{2}} u_{2} \\
& U_{d}\left(u_{1}, u_{2}, u_{3}\right)=\frac{M\left(2 I_{k}-3 d^{2}\right)}{I_{1}^{4}} u_{1}+\frac{2 d M}{I_{1}^{3}} u_{2}-\frac{M}{I_{1}^{2}} u_{3},
\end{aligned}
$$

and

$$
U_{I}\left(u_{1}, u_{2}, u_{3}, u_{4}\right)=\frac{2 d\left(2 d^{2}-3 I_{k}\right) M}{I_{1}^{5}} u_{1}+\frac{M\left(2 I_{k}-3 d^{2}\right)}{I_{1}^{4}} u_{2}+\frac{2 d M}{I_{1}^{3}} u_{3}-\frac{M}{I_{1}^{2}} u_{4}
$$

where, in this case, $I_{1}=I_{x}+M, M_{k}=k+M$ and $I_{k}=I_{1} M_{k}$. Note the structure appearing in the columns due to the recursive properties of the matrix. 
Non-zero informative input: Assuming that the input is informative enough, $\mathcal{O}$ is full rank for almost all values of $x$, i.e. given a persistently exciting input, the states will be informative enough to distinguish between the parameters, and hence, it is possible to identify all three parameters. However, note that this might not be true for all numerical values of the parameters. One, possibly unlikely case, is when all parameters except for $I_{x}$ are zero, then no input will affect the output.

No input: Assuming that the input is zero but that the output still contains information, then the maximum rank of $\mathcal{O}$ drops to 4 . This occurs, for instance, if the system has stored energy induced by perturbing it from its equilibrium and releasing it. The drop in rank is due to the linear dependency of the columns corresponding to the parameters. It can be seen in the row-reduced (normalized per column) form of the extended observability matrix

$$
\overline{\mathcal{O}}=\left[\begin{array}{c:c:c:c:c}
x_{1} & x_{2} & k & d & I_{x} \\
\hdashline & 0 & 0 & 0 & 0 \\
\hdashline 0 & 1 & 0 & 0 & 0 \\
\hdashline 0 & 0 & 1 & 0 & 1 \\
\hdashline 0 & 0 & 0 & 1 & \frac{M_{k}}{I_{1}} \\
\hdashline 0 & 0 & 0 & 0 & 0 \\
\vdots & \vdots & \vdots & \vdots & \vdots
\end{array}\right]
$$

where the same color denotes that the elements assume the same value. Hence, it is only possible to identify two-combinations of parameters simultaneously. This result is reasonable since it is a second order system without input, i.e. a time-series model of second order.

\section{Identifiability of the Original Model - General Case}

For the general case of (4.13), the output is $y=\left[\dot{\phi}, a_{s}\right]^{T}$ and the input is $u=a_{y}$. For the analysis in this section, it is again assumed that $a_{y}$ is known.

Non-zero informative input: For the general case, the row reduced extended observability matrix (normalized per column) has the following structure

$$
\overline{\mathcal{O}}=\left[\begin{array}{ccc:cc:cccccc}
x_{1} & x_{2} & k & d & I_{x} & M & z_{g} & I_{x, m} & m & z_{m} \\
\hline 1 & 0 & 0 & 0 & 0 & 0 & 0 & 0 & 0 & 0 \\
\hdashline 0 & 1 & 0 & 0 & 0 & 0 & 0 & 1 & 0 & 0 & \frac{1}{0} \\
\hdashline 0 & 0 & 1 & 0 & 0 & 1 & 1 & 0 & 1 & 1 \\
\hdashline 0 & 0 & 0 & 1 & 0 & \times & \times & 0 & \times & \times \\
\hdashline 0 & 0 & 0 & 0 & 1 & \times & \times & 1 & \times & \times \\
\hdashline 0 & 0 & 0 & 0 & 0 & 0 & 0 & 0 & 0 & 0 \\
\vdots & \vdots & \vdots & \vdots & \vdots & \vdots & \vdots & \vdots & \vdots & \vdots
\end{array}\right]
$$

Note that the matrix is symbolic, i.e. $\times$ corresponds to a symbolic expression. This row-reduced form shows that the maximum rank of the extended observability matrix $\mathcal{O}$ is 5 , assuming that the input is informative enough. This implies that at most 3 parameters can be uniquely identified. This is an expected result since (4.13) is a second order system with one external input. 
Furthermore, it is possible to see that certain combinations of parameters will create issues with identifiability. In principle, this is equivalent to creating submatrices for each one-, two- and three-combination of parameters of the form

$$
\mathcal{O}_{s}=\left[\begin{array}{c:c:c:c:c}
\frac{\partial h(x, u, \vartheta)}{\partial x_{1}} & \frac{\partial h(x, u, \vartheta)}{\partial x_{2}} & \frac{\partial h(x, u, \vartheta)}{\partial \vartheta_{1}} & \frac{\partial h(x, u, \vartheta)}{\partial \vartheta_{2}} & \frac{\partial h(x, u, \vartheta)}{\partial \vartheta_{3}} \\
\vdots & \vdots & \vdots & \vdots & \vdots \\
\frac{\partial h^{(4)}(x, u, \vartheta)}{\partial x_{1}} & \frac{\partial h^{(4)}(x, u, \vartheta)}{\partial x_{2}} & \frac{\partial h^{(4)}(x, u, \vartheta)}{\partial \vartheta_{1}} & \frac{\partial h^{(4)}(x, u, \vartheta)}{\partial \vartheta_{2}} & \frac{\partial h^{(4)}(x, u, \vartheta)}{\partial \vartheta_{3}}
\end{array}\right]
$$

and checking the rank condition of these sub-matrices.

Firstly, note that each column corresponding to a parameter in (4.18) is linearly independent of the columns corresponding to the states and hence, if only one parameter is unknown, it is identifiable.

Secondly, any two columns in (4.18) corresponding to the parameters are linearly independent of each other, except for the columns corresponding to the parameters $I_{x}$ and $I_{x, m}$. This implies that except for $I_{x}$ and $I_{x, m}$, any two-combination of parameters is identifiable.

Finally, the analysis reveals that any three-combination of parameters is identifiable, except for any three-combination of $\left\{I_{x}, M, z_{g}, I_{x, m}, m, z_{m}\right\}$ or any combination of parameters where both $I_{x}$ and $I_{x, m}$ are in the set. For instance, this means that if the entire system is known except for the additional load, it is not possible to estimate its inertia, mass and its center of mass at the same time.

These results are summarized in Table 4.1. It is worth noting that since the analysis is performed with a symbolic software, the parameters are assumed to have distinctly different values. Hence, identifiability might be lost for certain numerical values of the parameters.

No input: When the input $a_{y}=0$, the row-reduced extended observability matrix (normalized per column) reduces to

$$
\overline{\mathcal{O}}=\left[\begin{array}{cc:c:c:ccccccc}
x_{1} & x_{2} & k & d & I_{x} & M & z_{g} & I_{x, m} & m & z_{m} \\
\hdashline 1 & 0 & 0 & 0 & 0 & 0 & 0 & 0 & 0 & 0 \\
\hdashline 0 & 1 & 0 & 0 & 0 & 0 & 0 & 0 & 0 & 0 \\
\hdashline 0 & 0 & 1 & 0 & 1 & 1 & 1 & 1 & 1 & 1 \\
\hdashline 0 & 0 & 0 & 1 & \times & \times & \times & \times & \times & \times \\
\hdashline 0 & 0 & 0 & 0 & 0 & 0 & 0 & 0 & 0 & 0 \\
\vdots & \vdots & \vdots & \vdots & \vdots & \vdots & \vdots & \vdots & \vdots & \vdots
\end{array}\right]
$$

and hence, the maximum rank of $\mathcal{O}$ drops to 4 and dropping column by column shows that any two-combination of parameters is identifiable except for the combination of $I_{x}$ and $I_{x, m}$, assuming the output still is informative enough. The results are summarized in Table 4.1. 
Table 4.1: The results of the identifiability analysis for the general case of the approximate model. The exceptions are: a) Combinations including both the parameters $I_{x}$ and $I_{x, m}$. b) Any three-combination of $\left\{I_{x}, M, z_{g}, I_{x, m}, m, z_{m}\right\}$.

\begin{tabular}{cccc}
\hline Case & \# Parameters & Identifiable & Exceptions \\
\hline \multirow{3}{*}{$a_{y} \neq 0$} & 1 & Yes & - \\
& 2 & Yes & $\mathrm{a}$ \\
& 3 & Yes & $\mathrm{a}, \mathrm{b}$ \\
\hline \multirow{3}{*}{$a_{y}=0$} & 1 & Yes & - \\
& 2 & Yes & $\mathrm{a}$ \\
& 3 & No & - \\
\hline
\end{tabular}

\subsection{A Limited Sensor Approach - The Input Model}

One of the main issues with estimating the parameters of the system (4.13) is the unknown and highly influential acceleration $a_{y}$. Several approaches are possible:

(A) For sake of completeness, if $a_{y}$ was known or measured, it could be used as an input: $x=[\phi, \dot{\phi}]^{T}, y=\left[y_{1}, y_{2}\right]^{T}$ and $u=a_{y}$. The estimation would then be straightforward using, for example, a standard prediction-error method (PEM) if $a_{y}$ is known or an errors-in-variables (EIV) method if $a_{y}$ is measured with noise (Söderström, 1981; Ljung, 1999).

(B) The variable $a_{y}$ could be introduced as a state. However, this requires a model of how $a_{y}$ behaves. Possible alternatives are, for instance,

(a) an extended model with the states $x=\left[\phi, \dot{\phi}, a_{y}\right]^{T}$, the outputs $y=$ $\left[y_{1}, y_{2}\right]^{T}$, defined by (3.41) and (3.44), and where the model of $a_{y}$ is assumed to be a random walk.

(b) if the lateral velocity was measured, for instance using a GPS receiver, then the states could be chosen as $x=\left[\phi, \dot{\phi}, v_{y}, a_{y}\right]^{T}$ and the outputs $y=\left[y_{1}, y_{2}, v_{y}\right]^{T}$ defined by (3.41) and (3.44). The velocity $v_{y}$ obtained with the GPS receiver would then implicitly supply information about $a_{y}$.

(C) The variable $a_{y}$ is eliminated from the model, in analogy with a Luenberger reduced-order observer, using the known relation

$$
a_{s}=z_{s} \ddot{\phi}+g \phi-a_{y}
$$

defined in (3.43). Note that in reality $a_{s}$ is measured which leads to an EIV formulation.

Here, alternative $(\mathrm{C})$ is used since both $a_{y}$ and its model are unknown. Equation (3.43) is solved for $a_{y}$ and inserted into (4.10) resulting in

$$
I_{2} \ddot{\phi}=-k \phi-d \dot{\phi}-\left(M z_{g}+m z_{m}\right) a_{s}+\bar{\tau}
$$


with

$$
I_{2}=I_{x}+M z_{g}\left(z_{g}-z_{s}\right)+I_{x, m}+m z_{m}\left(z_{m}-z_{s}\right)
$$

and $a_{s}$ is now treated as the input.

\subsubsection{Identifiability of the Input Model}

The goal of the analysis in this section is to investigate if there is any change in the identifiability properties when $a_{s}$ is used as an input instead of the true input $a_{y}$. As a basis for comparison, the results for the original model (4.10) in Section 4.2.1 show what is possible to estimate given that the true input of the system is known.

In this section the output is chosen as $y=\dot{\phi}$ and the input is $u=a_{s}$, i.e. the measured tangential acceleration. By defining the states $x=[\phi, \dot{\phi}]^{T}$, the input model (4.21) and the measurement equation (3.41) can similarly to Section 4.2.1 be cast into the state-space form (neglecting the disturbances)

$$
\begin{aligned}
& \dot{x}=\left[\begin{array}{cc}
0 & 1 \\
-\frac{k}{I_{2}} & -\frac{d}{I_{2}}
\end{array}\right] x-\left[\begin{array}{c}
0 \\
\frac{M z_{g}+m z_{m}}{I_{2}}
\end{array}\right] a_{s} \\
& y=\left[\begin{array}{ll}
0 & 1
\end{array}\right] x
\end{aligned}
$$

where $I_{2}$ is defined by $(4.22)$.

Non-zero informative $a_{y}$ : As in Section 4.2.1, assuming that the true input $a_{y}$ is informative enough, the maximum rank of $\mathcal{O}$ is 5. Also the identifiable one-, two- and three-combinations of the parameters are the same. Hence, the identifiability properties of the original model are retained when using the mathematical reformulation.

No input $\left(a_{y}=0\right)$ : An apparent advantage with the reformulation (4.23) is that the unknown acceleration $a_{y}$ is replaced by the measured acceleration $a_{s}$. As defined in (3.43), the tangential acceleration is

$$
a_{s}=z_{s} \ddot{\phi}+g \phi-a_{y}
$$

and if the system is perturbed from its equilibrium, then $a_{s}$ will be non-zero for almost all $t$ even if $a_{y}$ is zero. At a first glance, this might seem beneficial since it suggests that the input $a_{s}$ is informative even though $a_{y}$ is zero. However, it is the input $a_{s}$ that is used in the analysis and it is assumed that the input can be chosen arbitrarily. The input is a linear combination of $a_{y}$ and the states and is thus not free to be chosen if $a_{y}$ is zero. By inserting the known relation (3.44), the maximum rank of $\mathcal{O}$ is 4 as in Section 4.2.1, again retaining the same identifiability properties as for the original model.

Result 4.1 In a local structural identifiability sense, nothing is gained or lost by using the input model (4.21) instead of the original model (4.10). 


\subsubsection{Model Analysis - A Closed-loop Viewpoint}

Even though the proposed method solves the problem with the unknown acceleration, it introduces some new challenges. One way of realizing this is to represent the system in a block diagram. The system (4.21) with the output $y=\dot{\phi}$ can be represented by

$$
\begin{gathered}
y=G(\mathrm{p}) a_{s}+H(\mathrm{p}) \bar{\tau}=G(\mathrm{p})\left(a_{s}+\tau\right), \text { with } \\
G(\mathrm{p})=\frac{\beta_{1} \mathrm{p}}{\mathrm{p}^{2}+\alpha_{1} \mathrm{p}+\alpha_{2}}, \quad H(\mathrm{p})=\frac{\gamma_{1} \mathrm{p}}{\mathrm{p}^{2}+\alpha_{1} \mathrm{p}+\alpha_{2}}=\frac{\gamma_{1}}{\beta_{1}} G(\mathrm{p}), \\
\alpha_{1}=\frac{d}{I_{2}}, \quad \alpha_{2}=\frac{k}{I_{2}}, \quad \beta_{1}=-\frac{M z_{g}+m z_{m}}{I_{2}}, \quad \gamma_{1}=\frac{1}{I_{2}}
\end{gathered}
$$

and with $I_{2}$ according to (4.22). The transfer operator from $a_{s}$ to $y$ is $G(\mathrm{p})$, $H(\mathrm{p})$ is the transfer operator from $\bar{\tau}$ to $y, \tau=G^{-1}(\mathrm{p}) H(\mathrm{p}) \bar{\tau}=\frac{\gamma_{1}}{\beta_{1}} \bar{\tau}$ and $\mathrm{p}$ is the differentiation operator.

At first, it appears to be straightforward to use a second order ARMAX structure, see Figure 4.6. However, $\bar{\tau}$ is not white, the input model (4.21) does not reveal the correlation between the input $a_{s}$ and the disturbance $\bar{\tau}$ neither does it reveal that $a_{s}$ is dependent on the output $y$.

To gain more insight, the original system (4.10) with the output $y$ can be expressed in terms of $G(\mathrm{p})$ together with the tangential acceleration's dependency on the true input $a_{y}$ and the output $y$.

Proposition 4.1 The original system (4.10) with the output $y$ is equivalent to $G(p)\left(a_{s}+\tau\right)$ in positive feedback with

$$
F_{y}(p)=\frac{z_{s} p^{2}+g}{p}
$$

and $-a_{y}$ as an external signal, see Figure 4.7.

Proof: The proof follows immediately from inserting the known input

$$
a_{s}=\frac{z_{s} \mathrm{p}^{2}+g}{\mathrm{p}} y-a_{y}=\left(z_{s} \mathrm{p}^{2}+g\right) \phi-a_{y}
$$

into (4.24) which after algebraic manipulations results in the original model (4.10).

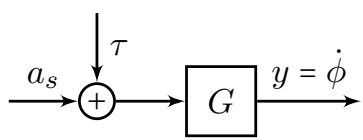

Figure 4.6: The system (4.24) represented in a block diagram showing the apparent simple structure. However, note that $a_{s}$ is dependent on $y$ according to (3.43) which also means that it is correlated with $\tau$. 


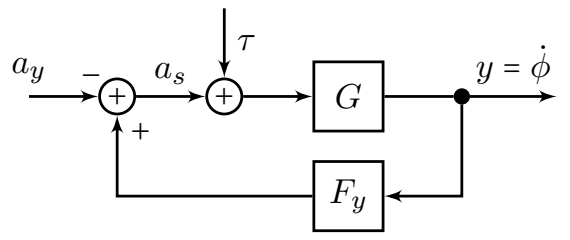

Figure 4.7: The system (4.10) expressed in terms of $G(p)$ shown as a block diagram. This representation reveals the dependencies in the system more clearly than Figure 4.6.

The structure revealed by this point of view shows that in principle, this is identification in closed-loop. This means that in case $a_{s}$, the input to $G(\mathrm{p})$, is persistently exciting, the input might not be sufficiently informative since $a_{y}$ is the true input to the system. It is well-known in system identification theory that the reference has to be persistently exciting and that a too simple linear feedback term does not contribute new information, see for instance, Söderström and Stoica (1989, Section 10.3), Ljung (1999, Section 13.4) or Bazanella et al. (2010). Note that the linear measurement equation is due to the assumption that the IMU only measures the tangential acceleration. If the IMU is rotated, it will also measure the normal or centripetal acceleration which dependence nonlinearly on the angular velocity. Hence, if the IMU is deliberately rotated, the feedback term might contribute information even if the true input is zero. This will however lead to a nonlinear estimation problem.

Furthermore, $\lim _{\mathrm{s} \rightarrow \infty} G(\mathrm{~s}) F_{y}(\mathrm{~s}) \neq 0$ which means that the loop contains a direct term. Hence, any accurate discrete approximation of this continuous-time system will also include a direct term. Assume that an accurate discretization method has been applied to (4.24), giving

$$
y_{t}=G_{d}(\mathrm{q}) u_{t}+H_{d}(\mathrm{q}) \bar{\tau}_{t}=G_{d}(\mathrm{q}) u_{t}+\bar{H}_{d}(\mathrm{q}) e_{t}
$$

where $\mathrm{q}$ is the shift operator. Also, assume that the discrete-time noise can be described as $\bar{\tau}_{t}=H_{1}(\mathbf{q}) e_{t}$ where $e_{t}$ is white noise and that $H_{1}(\mathrm{q})$ is a stable discrete-time linear filter. Even if the correct noise model $H_{d}(\mathbf{q}) H_{1}(\mathrm{q})$ is known, the residual

$$
\varepsilon_{t}=H_{1}^{-1}(\mathrm{q}) H_{d}^{-1}(\mathrm{q})\left[y_{t}-G_{d}(\mathrm{q}) u_{t}\right]=H_{1}^{-1}(\mathrm{q}) \bar{\tau}_{t}
$$

and the input-dependent term $H_{1}^{-1}(\mathrm{q}) H_{d}^{-1}(\mathrm{q}) G_{d}(\mathrm{q}) u_{t}=c H_{1}^{-1}(\mathrm{q}) u_{t}$, where $c$ is a constant, will be correlated and the consequence is a risk that the parameter estimator will be biased if a direct closed-loop identification method is applied straightforwardly.

Finally, when the true $a_{s}$ and $y$ are not known but measured according to Section 3.6.1, the input will be noisy which results in a different noise model, i.e. this is a closed-loop EIV problem, see Figure 4.8 . 


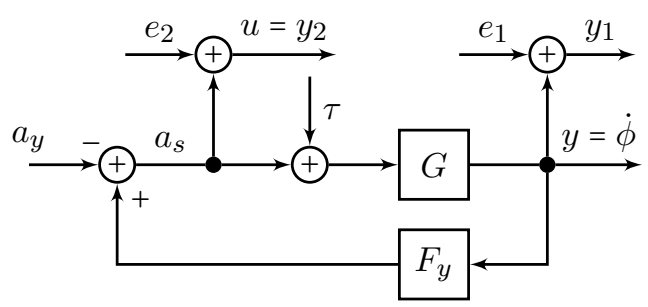

Figure 4.8: A block diagram of the system (4.10) expressed in terms of $G(p)$ which shows the relation between $y, a_{s}$ and $a_{y}$. The input $a_{s}$ and the output $y$ are measured with noise according to Section 3.6.1. This means that identification of $G(p)$ using $y_{2}$ as input and $y_{1}$ as output is similar to a closed loop EIV problem.

\subsubsection{Discretization and Connection to the Physical Parameters}

All measurements are taken at discrete-time instances and a discrete-time model is needed to relate the measurements to the parameters. Several different discretization methods can be used but here, the transfer functions (4.25) are discretized with the bilinear transform $\mathrm{p}=\frac{2}{T} \frac{\mathrm{q}-1}{\mathrm{q}+1}$ where $T$ is the sample period.

The benefits of the bilinear transform are the simple algebraic relation between $\mathrm{p}$ and $\mathrm{q}$ while still being fairly accurate for short sampling periods and that the resulting discrete-time model is stable for all sampling periods. Hence, this discretization approach makes it possible to have a direct connection between the discrete-time linear parameters and the physical parameters. This is beneficial since it allows an easy way to decrease the variance of the parameter estimates by introducing constraints in the estimation procedure, which will be discussed further in Sections 4.3.4 and 4.4.1.

Applying the bilinear transform to (4.25) gives

$$
\begin{array}{r}
G_{d}(\mathbf{q})=\frac{\bar{\beta}_{0}\left(1-\mathrm{q}^{-2}\right)}{1+\bar{\alpha}_{1} \mathrm{q}^{-1}+\bar{\alpha}_{2} \mathrm{q}^{-2}}, \quad H_{d}(\mathrm{q})=\frac{\bar{\gamma}_{0}\left(1-\mathrm{q}^{-2}\right)}{1+\bar{\alpha}_{1} \mathrm{q}^{-1}+\bar{\alpha}_{2} \mathrm{q}^{-2}}, \text { with } \\
\bar{\alpha}_{1}=\frac{2 \alpha_{2} T^{2}-8}{\bar{I}_{2}}, \quad \bar{\alpha}_{2}=\frac{4-2 \alpha_{1} T+\alpha_{2} T^{2}}{\bar{I}_{2}}, \bar{\beta}_{0}=\frac{2 \beta_{1} T}{\bar{I}_{2}}, \quad \bar{\gamma}_{0}=\frac{2 \gamma_{1} T}{\bar{I}_{2}}
\end{array}
$$

and $\bar{I}_{2}=4+2 \alpha_{1} T+\alpha_{2} T^{2}$. The discrete-time model can now be written according to (4.28) or as the linear regression

$$
y_{t}=\varphi_{t}^{T} \vartheta+\tau_{t}
$$

where $\varphi_{t}^{T}=\left[-y_{t-1},-y_{t-2}, u_{t}-u_{t-2}\right], \vartheta=\left[\bar{\alpha}_{1}, \bar{\alpha}_{2}, \bar{\beta}_{0}\right]^{T}$ and $\tau_{t}=\bar{\gamma}_{0}\left(\bar{\tau}_{t}-\bar{\tau}_{t-2}\right)$. Note that $\tau_{t}$ is a colored noise process.

The inverse transform gives the continuous-time parameters

$$
\alpha_{1}=-\frac{4}{T} \frac{\bar{\alpha}_{2}-1}{1-\bar{\alpha}_{1}+\bar{\alpha}_{2}}, \alpha_{2}=\frac{4}{T^{2}} \frac{1+\bar{\alpha}_{1}+\bar{\alpha}_{2}}{1-\bar{\alpha}_{1}+\bar{\alpha}_{2}}, \beta_{1}=\frac{8}{T} \frac{\bar{\beta}_{0}}{1-\bar{\alpha}_{1}+\bar{\alpha}_{2}} \text { and } \gamma_{1}=\frac{8}{T} \frac{\bar{\gamma}_{0}}{1-\bar{\alpha}_{1}+\bar{\alpha}_{2}}
$$

Given the continuous-time linear parameters, the physical parameters can be found 
through a nonlinear transformation. In principle, a transformation is available for all identifiable combinations of parameters in Section 4.2 .1 by solving selected parts of (4.26) for the wanted parameters. Here, two important cases are listed. Firstly, if $I_{x}, k$ and $d$ are unknown, then the numerator of $\beta_{1}$ is known which gives the solution

$$
\begin{aligned}
d & =\frac{\alpha_{1}}{\beta_{1}}\left(-M z_{g}-m z_{m}\right) \\
k & =\frac{\alpha_{2}}{\beta_{1}}\left(-M z_{g}-m z_{m}\right) \\
I_{x} & =\frac{-M z_{g}-m z_{m}-\beta_{1}\left[M z_{g}\left(z_{g}-z_{s}\right)+I_{x, m}+m z_{m}\left(z_{m}-z_{s}\right)\right]}{\beta_{1}}
\end{aligned}
$$

Secondly, if the additional mass $m$ and center of mass $z_{m}$ are unknown then the transform is given by, for instance, solving $\alpha_{1}$ and $\beta_{1}$ in (4.26) for $m$ and $z_{m}$. The solution is given by

$$
\begin{gathered}
m=-\frac{\left(\beta_{1} d+\alpha_{1} M z_{g}\right)^{2}}{\alpha_{1}^{2}\left(I_{x}+M z_{g}^{2}\right)+\alpha_{1} d\left(\beta_{1} z_{s}-1\right)} \\
z_{m}=\frac{\alpha_{1}\left(I_{x}+M z_{g}^{2}\right)+d\left(\beta_{1} z_{s}-1\right)}{\alpha_{1} M z_{g}+\beta_{1} d}
\end{gathered}
$$

It should be noted that other discretization methods, such as, forward or backward Euler, can be used in a similar manner.

\subsubsection{Obeying Nonlinear Parameterization Using Constraints}

As discussed in Section 4.3.1, only a subset of all parameters can be estimated. The transfer function (4.30) has three free parameters, i.e. three degrees of freedom to fit the model to the data. When all three parameters are estimated, this gives a model that is more flexible than permitted with the consequence of increased variance of the parameter estimates (Ljung, 1999). The dependency between the linear parameters is complex since they are dependent through the original nonlinear parameterization. Fortunately, for certain parameter combinations, it is possible to use linear constraints together with (4.30) and still obey the original nonlinear parameterization. The flexibility is thus reduced which results in a reduced estimator variance. Arbitrary nonlinear constraints can also be used but since the model given by (4.30) can be written as a linear regression, using linear constraints makes the estimation algorithms computationally cheaper. Below, a few examples of these linear constraints are presented. These are all based on mathematical manipulations involving known numerators or known coefficients. In all cases, the discrete linear parameter vector is $\vartheta=\left[\bar{\alpha}_{1}, \bar{\alpha}_{2}, \bar{\beta}_{0}\right]^{T}$.

If only $I_{x}$ and $d$ are unknown, both $\alpha_{2}$ and $\beta_{1}$ have known numerators and the quotient

$$
-\frac{M z_{g}+m z_{m}}{k}=\frac{\beta_{1}}{\alpha_{2}}=\frac{2 T \bar{\beta}_{0}}{1+\bar{\alpha}_{1}+\bar{\alpha}_{2}}
$$


is thus known giving the constraint

$$
\left[\begin{array}{lll}
-\frac{M z_{g}+m z_{m}}{k} & -\frac{M z_{g}+m z_{m}}{k} & -2 T
\end{array}\right] \vartheta=\frac{M z_{g}+m z_{m}}{k}
$$

Similarly, in the case where only $I_{x}$ and $k$ are unknown, the numerators of $\alpha_{1}$ and $\beta_{1}$ are known and the quotient

$$
-\frac{d}{M z_{g}+m z_{m}}=\frac{\alpha_{1}}{\beta_{1}}=\frac{1-\bar{\alpha}_{2}}{2 \bar{\beta}_{0}}
$$

between them is known giving the constraint

$$
\left[\begin{array}{lll}
0 & 1 & -2 \frac{d}{M z_{g}+m z_{m}}
\end{array}\right] \vartheta=1
$$

If $k$ and $d$ are the only unknown parameters then

$$
\beta_{1}=\frac{8}{T} \frac{\bar{\beta}_{0}}{1-\bar{\alpha}_{1}+\bar{\alpha}_{2}}
$$

is known giving the constraint

$$
\left[\begin{array}{lll}
\beta_{1} T & -\beta_{1} T & 8
\end{array}\right] \vartheta=\beta_{1} T
$$

If any two-combination of $\left\{I_{x}, M, z_{g}, I_{x, m}, m, z_{m}\right\}$ is unknown, the quotient

$$
\frac{d}{k}=\frac{\alpha_{1}}{\alpha_{2}}=\frac{T\left(1-\bar{\alpha}_{2}\right)}{1+\bar{\alpha}_{1}+\bar{\alpha}_{2}}
$$

is known which gives the constraint

$$
\left[\begin{array}{lll}
\frac{d}{k} & \frac{d}{k}+T & 0
\end{array}\right] \vartheta=T-\frac{d}{k}
$$

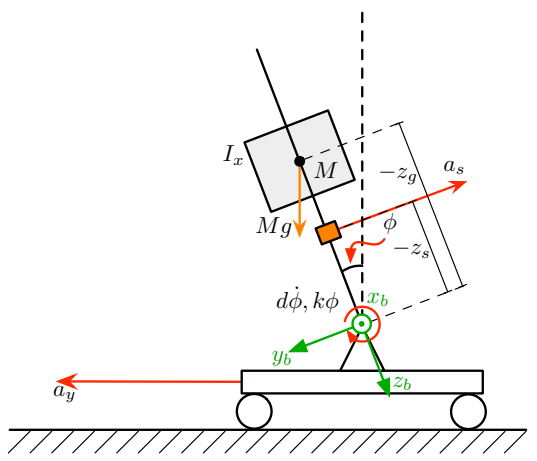

(a) $Z_{n}$ (Nominal)

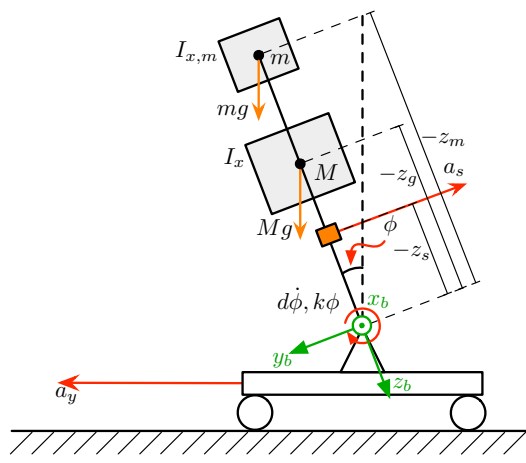

(b) $Z_{l}$ (Additional load)

Figure 4.9: Data are collected from two different load cases, a nominal case giving the dataset $Z_{n}$ and a case with additional load giving the dataset $Z_{l}$. 


\subsection{Multi-Stage Method}

In order to estimate the change in mass and the change in the center of mass on a real system, several sources of information and datasets have to be combined to overcome the identifiability limitations. One approach to solve this is to use the multi-stage identification procedure described in this section. Another approach is the joint estimation procedure in Section 5.5. If no a priori information is known about the system parameters, this is indeed necessary since a maximum of three parameters can be identified simultaneously according to the results of Section 4.3.1. To counteract the force-mass ambiguity, a nominal and a loaded dataset are used, see Figure 4.9. The datasets are defined by

$$
Z_{n}=\left(y_{t}, u_{t}, \delta_{t}\right)_{t=1+t_{n}}^{N_{n}+t_{n}} \text { and } Z_{l}=\left(y_{t}, u_{t}, \delta_{t}\right)_{t=1+t_{l}}^{N_{l}+t_{l}}
$$

where $N_{n}$ and $N_{l}$ denotes the lengths of the datasets. Note that these datasets are not collected at the same time, hence the different starting times $t_{n}$ and $t_{l}$. They are related through the physical parameters

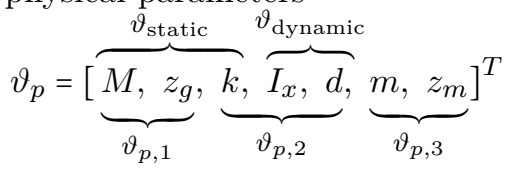

which can be divided in several ways, e.g. $\vartheta_{p, 1}, \vartheta_{p, 2}$ and $\vartheta_{p, 3}$ or $\vartheta_{\text {static }}, \vartheta_{\text {dynamic }}$ and $\vartheta_{p, 3}$. In the multi-stage method, these are estimated sequentially, where the estimate from the previous step is used in the next step. The division into subsets is both dependent on the identifiability properties of the model and on the type of sensors available.

Due to identifiability issues, in the first step, two of the parameters $M, z_{g}$ and $I_{x}$ need to be known or estimated. This step involves some special kind of experiment. For instance, $\vartheta_{p, 1}$, i.e. the mass and center of mass, can be determined by specific procedures such as the tilting procedure described in Section 6.2.1 or $\vartheta_{\text {static }}$ can be determined using the ballasting approach described in Appendix A.

In the second step, depending on the parameters estimated in the first step, either the dynamic parameters $\vartheta_{\text {dynamic }}$ or $\vartheta_{p, 2}$ are estimated using the nominal dataset.

In the third step, all the parameters of the nominal case are known or estimated and the loaded dataset is used to estimate $\vartheta_{p, 3}$ or to monitor changes in some other parameters. Note that the real benefit is that the loaded dataset can be collected at any point in time and the change in mass and change in center of mass can be estimated during normal operation.

The different steps can be formalized. For example, if the parameters $\vartheta_{p, 1}$, i.e. $M$ and $z_{g}$, of the nominal system are estimated using some special procedure, then Approach 4.1 can be used to compute an estimate of the parameters. This can of course be done for the static and dynamic parameters in (4.45) or be extended to other model structures where more steps might be required. Note that $z_{s}$ is assumed to be known. The numbering of Approach 4.1 starts at 2 to indicate that a step have to be performed prior to using the approach. 


\section{Approach 4.1 (Example of the multi-stage method)}

2. The estimate $\hat{\vartheta}_{p, 1}$ is used together with the nominal dataset to estimate $I_{x}$, $d$ and $k$ by computing

$$
\begin{gathered}
\hat{\vartheta}_{p, 2}=\underset{\vartheta_{p, 2}}{\operatorname{argmin}} V_{2}\left(\hat{\vartheta}_{p, 1}, \vartheta_{p, 2}, Z_{n}\right) \\
\text { s.t. } h_{2}\left(\hat{\vartheta}_{p, 1}, \vartheta_{p, 2}\right)=0
\end{gathered}
$$

where $V_{2}\left(\vartheta, Z_{n}\right)$ is a nonlinear criterion function and $h_{2}(\cdot)=0$ is a possibly nonlinear equality constraint.

3. Finally, using the loaded dataset and the estimates $\hat{\vartheta}_{p, 1}$ and $\hat{\vartheta}_{p, 2}$, the changes in mass $m$ and the change in center of mass $z_{m}$ are estimated by computing

$$
\begin{gathered}
\hat{\vartheta}_{p, 3}=\underset{\vartheta_{p, 3}}{\operatorname{argmin}} V_{3}\left(\hat{\vartheta}_{p, 1}, \hat{\vartheta}_{p, 2}, \vartheta_{p, 3}, Z_{l}\right) \\
\text { s.t. } h_{3}\left(\hat{\vartheta}_{p, 1}, \hat{\vartheta}_{p, 2}, \vartheta_{p, 3}\right)=0
\end{gathered}
$$

where $V_{3}\left(\vartheta, Z_{l}\right)$ is a nonlinear criterion function and $h_{3}(\cdot)=0$ is a possibly nonlinear equality constraint.

\subsubsection{Linear Criterion with Linear Constraints}

As discussed in Section 4.3.3, the input model can be written as a linear regression and if the criterion functions are quadratic in the linear discrete-time parameters, Steps 2 and 3 in Approach 4.1 can be performed by solving a least-squares problem with linear constraints and then transforming the obtained linear parameters. In these cases, given that $\hat{\vartheta}_{p, 1}$ and $z_{s}$ are known, Approach 4.1 can be further specified to Approach 4.2 which is computationally inexpensive. The real benefit of this solution is that each step can be efficiently solved using factorization, see Golub and Van Loan (1996, Ch. 12).

The choices of $\Phi^{i}$ and $Y^{i}, i=\{n, l\}$ dictate the properties of the solutions in Steps 2a and 3a of Approach 4.1. Here, two choices are listed:

1. The least-squares estimator given by

$$
\Phi^{i}=\frac{1}{N}\left[\begin{array}{c}
\varphi_{1}^{T} \\
\vdots \\
\varphi_{N}^{T}
\end{array}\right] \text { and } Y^{i}=\frac{1}{N}\left[\begin{array}{c}
y_{1} \\
\vdots \\
y_{N}
\end{array}\right]
$$

However, note that the noise model is incorrect due to the ARX structure assumed to form the LS estimator.

2. The (extended) instrumental variable estimator given by

$$
\Phi^{i}=\frac{1}{N}\left[\begin{array}{lll}
\zeta_{1} & \cdots & \zeta_{N}
\end{array}\right]\left[\begin{array}{c}
\bar{\varphi}_{1}^{T} \\
\vdots \\
\bar{\varphi}_{N}^{T}
\end{array}\right] \text { and } Y^{i}=\frac{1}{N}\left[\begin{array}{lll}
\zeta_{1} & \cdots & \zeta_{N}
\end{array}\right]\left[\begin{array}{c}
\bar{y}_{1} \\
\vdots \\
\bar{y}_{N}
\end{array}\right]
$$

where $\zeta_{t}$ is the vector of instruments, $\bar{y}_{t}=L(\mathbf{q}) y_{t}, \bar{\varphi}_{t}^{T}=L(\mathbf{q}) \varphi_{t}^{T}$ and $L(\mathbf{q})$ is a stable prefilter. The prefilter is here either set a priori or estimated in an iterative scheme, and for a minimum variance estimator the prefilter should be equal to the inverse noise model, see Section 2.4.2. 


\section{Approach 4.2 (The linear multi-stage method)}

2a. The estimate $\hat{\vartheta}_{p, 1}$ is used together with the nominal dataset $Z_{n}$ to compute

$$
\begin{gathered}
\hat{\vartheta}_{2}=\underset{\vartheta}{\operatorname{argmin}}\left\|\Phi^{n} \vartheta-Y^{n}\right\|_{2}^{2} \\
\text { s.t. } B_{2} \vartheta=b_{2}
\end{gathered}
$$

where $B_{2}$ and $b_{2}$ are either

i. empty if $I_{x}, k$ and $d$ are unknown.

ii. given by (4.37) if $k$ is known.

iii. given by (4.39) if $d$ is known.

2b. $\hat{\vartheta}_{p, 2}$ is given by (4.33) and (4.34).

3a. The loaded dataset $Z_{l}$ and the estimates $\hat{\vartheta}_{p, 1}$ and $\hat{\vartheta}_{p, 2}$ are used to compute

$$
\begin{gathered}
\hat{\vartheta}_{3}=\underset{\vartheta}{\operatorname{argmin}}\left\|\Phi^{l} \vartheta-Y^{l}\right\|_{2}^{2} \\
\text { s.t. } B_{3} \vartheta=b_{3}
\end{gathered}
$$

where $B_{3}$ and $b_{3}$ are given by (4.43).

3b. Finally, the changes in mass $m$ and the center of mass $z_{m}$ are given by (4.33) and (4.35).

Note that the physical parameters are replaced with the estimates where necessary, for instance, in (4.37).

\section{Choice of Instruments for the Extended IV Estimator}

As discussed in Section 2.4.2, any instruments that fulfill the conditions in (2.30) will guarantee consistency, but the variance properties of the parameter estimator are greatly affected by the choice of instruments and prefilter (Forssell, 1999; Ljung, 1999). Further insight about the choice of prefilter, the optimal instruments and approximate optimal instruments for a closed-loop system is discussed in Section 2.4.2. These results are all based on using the noise model and an external reference signal, instead of the input, to create the optimal instruments. Even though none of these sources consider optimal instruments for the case with noisy input and output data according to Figure 4.8, it seems likely that it should be possible to decrease the variance by using a similar strategy also in the present case. Preferably, the variable $a_{y}$ should be used as the external reference, but it is unknown and instead an estimate $\hat{a}_{y}$ is used. As mentioned in Section 4.2 and shown in Figure 4.3 and Figure 4.10, $a_{y}$ is correlated with the external reference $\delta$ through the relation $a_{y}=F_{\delta}(\mathrm{p}) \delta$ but $F_{\delta}(\mathrm{p})$ is assumed to be unknown and hence, an estimate is used according to

$$
\hat{a}_{y, t}=\hat{F}_{\delta, d}(\mathrm{q}) \delta_{t}
$$




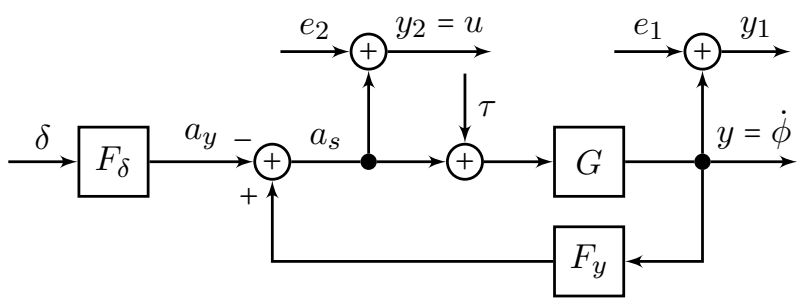

Figure 4.10: The system (4.10) expressed in terms of $G(p)$ with the external reference signal $\delta$.

Here, the instruments are chosen as

$$
\zeta_{t}=L(\mathrm{q})\left[\begin{array}{lllllllll}
\hat{a}_{y, t} & \cdots & \hat{a}_{y, t-n_{\delta}-1} & \hat{y}_{t} & \cdots & \hat{y}_{t-n_{y}-1} & \hat{a}_{s, t} & \cdots & \hat{a}_{s, t-n_{u}-1}
\end{array}\right]^{T}
$$

where $L(\mathrm{q})$ is a prefilter, $(\cdot)_{t}$ is the signal $(\cdot)$ at time $t$,

$$
\hat{y}_{s, t}=\frac{G_{d}(\mathbf{q}, \hat{\vartheta})}{1-G_{d}(\mathbf{q}, \hat{\vartheta}) F_{y}(\mathbf{q})} \hat{a}_{y, t} \text { and } \hat{a}_{s, t}=\frac{1}{1-G_{d}(\mathbf{q}, \hat{\vartheta}) F_{y}(\mathbf{q})} \hat{a}_{y, t}
$$

are the simulated output and input, respectively, and $\hat{\vartheta}$ is an estimate of the parameters. The constants $n_{\delta}, n_{y}$ and $n_{u}$ are the number of time lags (including the non-delay signal) included in $\zeta_{t}$ for the reference signal, the input and the output, respectively, i.e. $n_{\delta}=0$ means that $\hat{a}_{y, t}$ is not included in $\zeta_{t}$.

\subsection{Experimental Verification}

In this section, two experiments are presented. The first one is based on the simulation study presented in Linder et al. (2014b) and the second one is based on data from a modified inverted pendulum process presented in Linder et al. (2014a).

The goals of the first simulation study are to show the impact of measurement noise and to verify the applicability of the proposed method. The goals of the second experiment are to decrease the variance of the estimator and to show the applicability on real data.

\subsubsection{Simulation Study}

To investigate the properties of the suggested method, it was applied to simulated data from the pendulum system without any process disturbance acting on it, i.e. $\bar{\tau}$ is zero. The dataset was synthesized with the approximate model (4.13). The signal $a_{y}$ was formed by feeding the reference signal $\delta$ through the transfer function $F_{\delta}(\mathrm{s})=\frac{1}{0.2 \mathrm{~s}+1}$. The true parameters are listed in Table 4.2.

Two datasets were created, one with nominal mass and one with additional mass. Both have 150000 data points, corresponding to $25 \mathrm{~min}$ of data and a sampling period of $0.01 \mathrm{~s}$. White zero-mean Gaussian measurement noise with a standard deviation of 0.01 was added to the simulated measurement signals, where the 
Table 4.2: Parameters used in the simulation. Note that the additional mass is less than $1 \%$ of the total mass. For an explanation of the parameters see Figure 4.2 on page 36 .

\begin{tabular}{cccccccc}
\hline$M$ & $z_{g}$ & $I_{x}$ & $d$ & $k$ & $m$ & $z_{m}$ & $z_{s}$ \\
\hline $22.04 \mathrm{~kg}$ & $-0.018 \mathrm{~m}$ & $0.1385 \mathrm{kgm}^{2}$ & $0.1067 \mathrm{Nms}$ & $10.39 \mathrm{Nm}$ & $0.2 \mathrm{~kg}$ & $-0.274 \mathrm{~m}$ & $-0.218 \mathrm{~m}$ \\
\hline
\end{tabular}

amplitude of the noise was chosen to be similar to the Xsens IMU in Section 3.6.1. Note that this is actually a higher noise level on the gyro than in the real sensor. The noisy signals were subjected to a preprocessing stage where the signals were filtered through a zero-phase FIR equiripple low-pass filter of order 20 with a cut-off frequency of $0.5 \mathrm{~Hz}$. This filtering is similar to what would be performed on data collected from a real IMU. Note that this preprocessing should not be confused with the prefilter in Section 4.4.1. Figure 4.11(a) shows the first $110 \mathrm{~s}$ of the nominal data set (with no additional mass) and Figure 4.11(b) presents a more detailed view of a typical behavior of the system. The black boxes in Figure 4.11(a) correspond to the data shown in Figure 4.11(b).

The parameters were estimated using the IV method with the noise model

$$
\bar{H}_{d}(\mathrm{q})=\frac{1}{A(\mathrm{q})}
$$

where $A(\mathrm{q})$ is the denominator of $G_{d}(\mathbf{q})$. This gives the prefilter $L(\mathbf{q})=1$, see Section 2.4.2 for a discussion of the relation between the noise model and the prefilter. Furthermore, the transfer function $\hat{F}(\mathrm{~s})=\frac{1}{0.1 \mathrm{~s}+1}$ was used to form $\hat{a}_{y}$. Note that the time constant is different from the true one. For comparison, a constrained least-squares approach was also used to estimate the parameters.

In Step 2 of Algorithm 4.2, M, $z_{g}$ and $k$ were assumed to be known and $I_{x}$ and $d$ were estimated using the linear constraint (4.37). In Step 3, $m$ and $z_{m}$ were estimated using the linear constraint (4.43). A Monte Carlo experiment with 10000 runs was performed.

The resulting parameters from Step 2a in Approach 4.2 can be seen in Figure 4.12(a) and the corresponding physical parameters from Step $2 \mathrm{~b}$, i.e. $I_{x}$ and $d$, can be seen in Figure 4.12(b)-(c). For both the physical parameters, the LS estimator has less variance but is biased. However, note that the bias is in the order of $10^{-3}$ for the $I_{x}$ estimator and in the order of $10^{-2}$ for the $d$ estimator. These are both small relative to the true values.

The results from the third and final stage, i.e. estimation of $m$ and $z_{m}$, can be seen in Figure 4.13(b)-(c). Figure 4.13(b) shows the result for the mass $m$ and Figure 4.13(c) shows the result for the distance $z_{m}$ from the center of rotation. In

this stage, the estimates, i.e. $\hat{I}_{x}$ and $\hat{d}$, from the previous stage, were used to replace the true parameters. Again, the IV estimator gave a smaller bias than the LS estimator for both $m$ and $z_{m}$ but a larger variance. For the corresponding discretetime parameters, see Figure 4.13(a). Note the increased variance compared to Figure 4.12(a). This was probably a result of that the estimator was less sensitive with respect to these parameters. However, this is something that needs further investigation. 

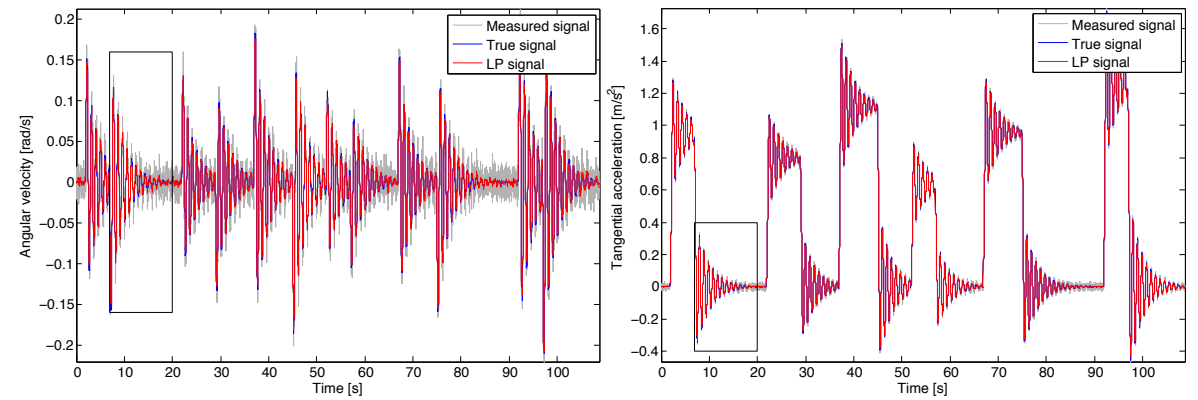

(a) The first $110 \mathrm{~s}$ of the nominal dataset.
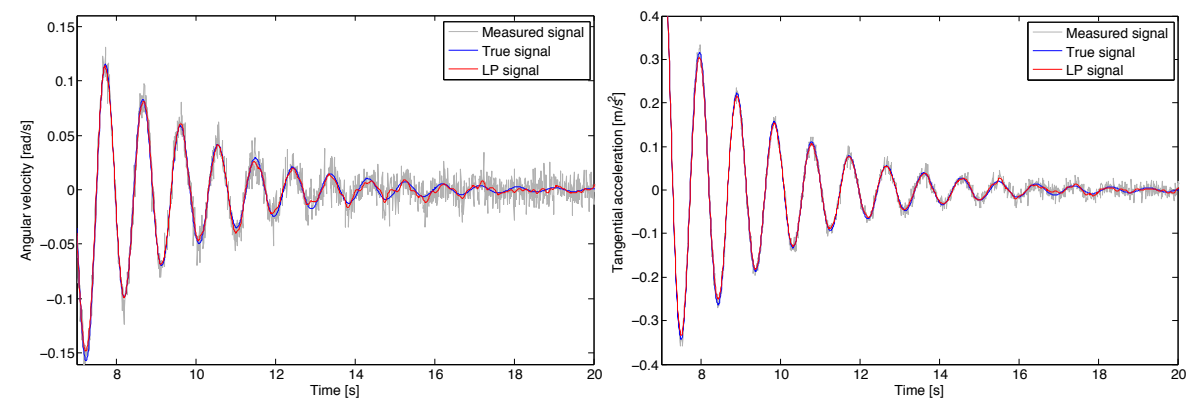

(b) A detailed view of data showing a typical behavior. This data corresponds to the black boxes marked in Figure 4.11(a).

Figure 4.11: The nominal dataset that was used in the identification. The measured signal is shown in gray, the true signal is blue and the measured signal after the LP filter was applied is dashed red. The left plot shows the angular velocity and the right plot shows the acceleration measurement.

According to theory, the estimated discrete-time linear parameters in the IV method will tend to the true ones as the number of data points goes to infinity (Söderström and Stoica, 1989). With the suggested IV estimator and the data length used, there was an obvious variation in the estimated physical parameters. This was probably due to the variance properties of the IV estimator and the sensitivity of the nonlinear transformation to the discrete-time linear parameters.

However, it should be emphasized that the increase in mass was less than $1 \%$ of the total mass. Hence, this simple version of the IV estimator was performing quite well since it actually estimated the mass above zero in all cases, within $\pm 0.1 \mathrm{~kg}$ in $85 \%$ of the cases and above $0.1 \mathrm{~kg}$ in $95 \%$ of the cases. Hence, the estimator captured the change in mass quite well. It was also interesting to note that even though the LS estimator in the previous stage was biased, the estimator for mass and center of mass had low bias and variance. In this limited experiment, only measurement noise was considered and the real benefit of the IV method is when both the input and output are correlated with the same noise source. 

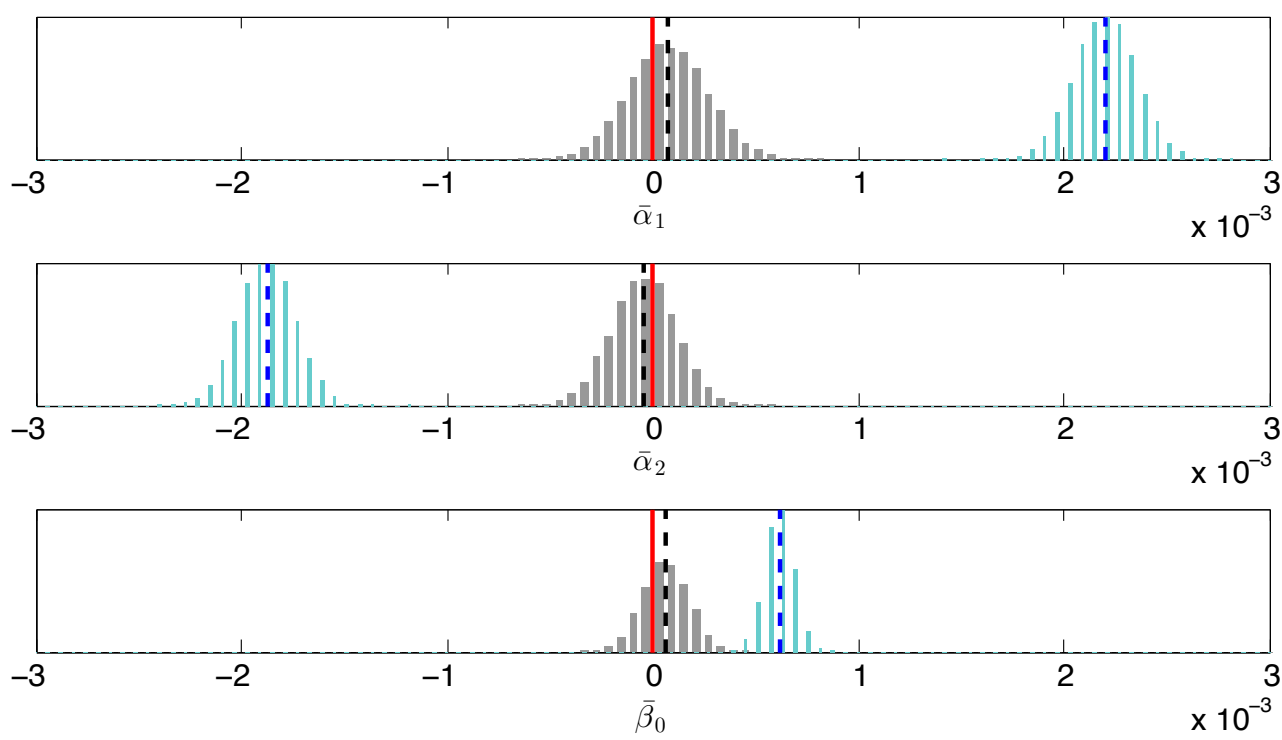

(a) The discrete linear parameters from Step 2a. From the top, $\bar{\alpha}_{1}$, $\bar{\alpha}_{2}$ and $\bar{\beta}_{0}$.

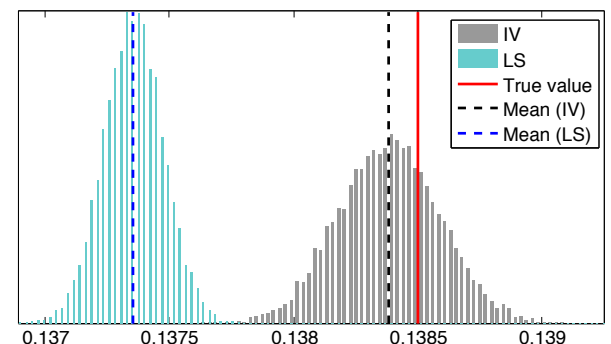

(b) The results for $I_{x}$ from Step $2 b$

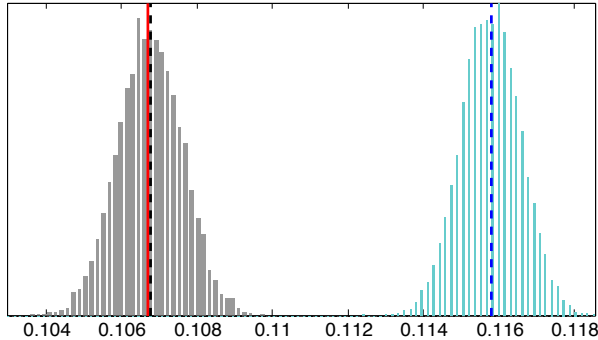

(c) The result for $d$ from Step $2 b$

Figure 4.12: The histograms of a Monte Carlo experiment with 10000 runs for Step 2 of Approach 4.2. The red line corresponds to the true value, the blue bars correspond to the LS method, the dashed blue line corresponds to the mean of the LS estimator, the gray bars correspond to the IV method and the dashed black line corresponds to the mean of the IV estimator. Note that the $L S$ estimator has smaller variance but larger bias. 

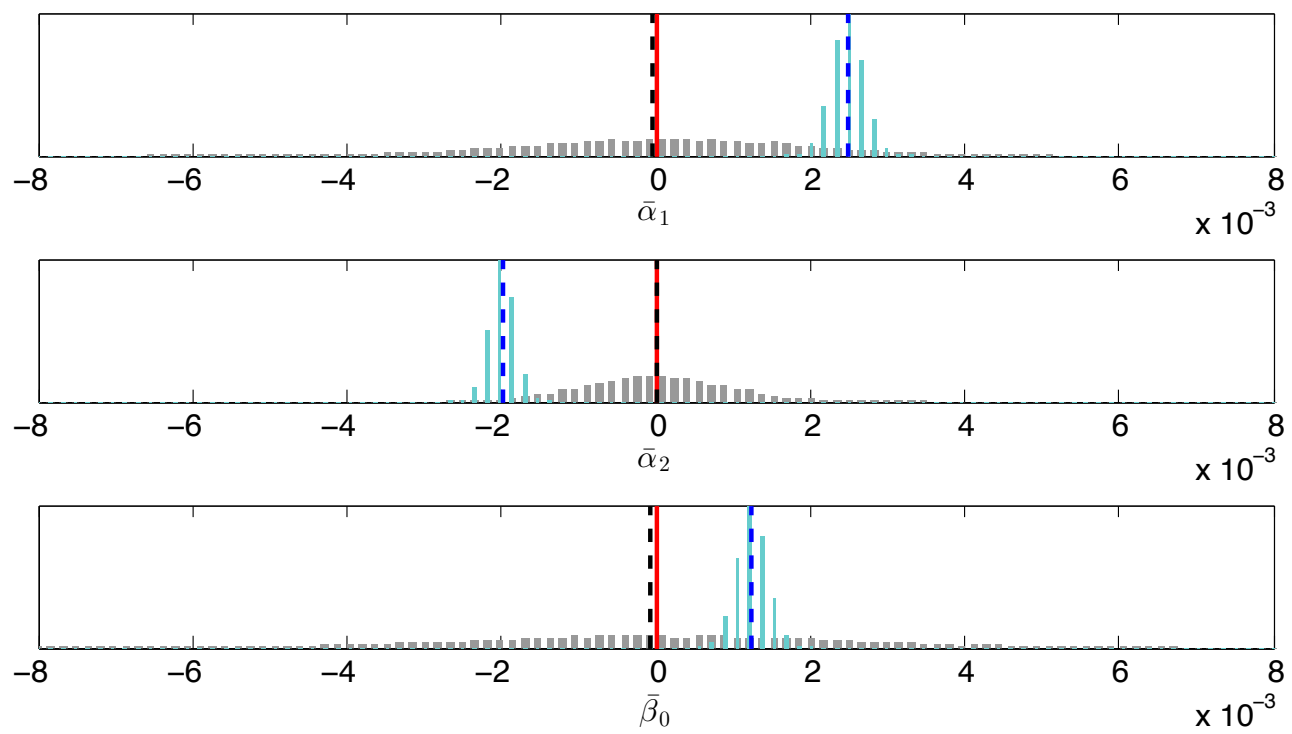

(a) The discrete linear parameters from Step 3a. From the top, $\bar{\alpha}_{1}, \bar{\alpha}_{2}$ and $\bar{\beta}_{0}$. Note the increase in variance of the $I V$ estimator in Comparison to Figure 4.12(a).

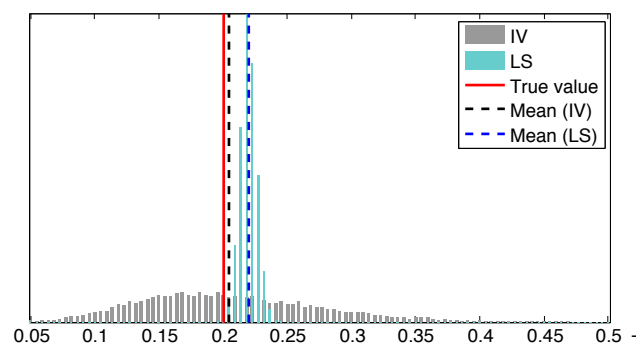

(b) The results for $m$ from Step $3 b$.

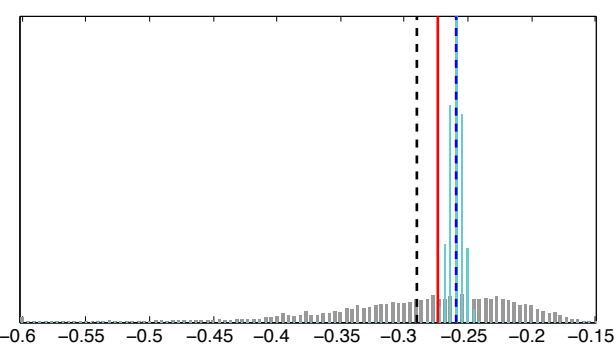

(c) The result for $z_{m}$ from Step $3 b$.

Figure 4.13: The histograms of a Monte Carlo experiment with 10000 runs for Step 3 of Approach 4.2. The red line corresponds to the true value, the blue bars correspond to the LS method, the dashed blue line corresponds to the mean of the LS estimator, the gray bars correspond to the IV method and the dashed black line corresponds to the mean of the IV estimator. Note that the $L S$ estimator has smaller variance but larger bias. 


\subsubsection{An Inverted Pendulum System}

This section describes an inverted pendulum experiment, together with results from the estimation procedure and an analysis of the results. The benefit of the experimental setup was that the system was very similar to the approximate model (4.10) and it could thus be used to validate the proposed method.

The experiment was based on real data collected from IMUs that were attached to an inverted pendulum modified with extra weights and springs to stabilize the pendulum. The system that was used in this experiment can be seen in Figure 4.14. The system consisted of a cart, six weights with adjustable positions, four springs and a mounting plate for the IMU. The known or measured masses, shapes and positions of each component were used to calculate the total inertia of the pendulum using theoretical results from basic mechanics. The weights and

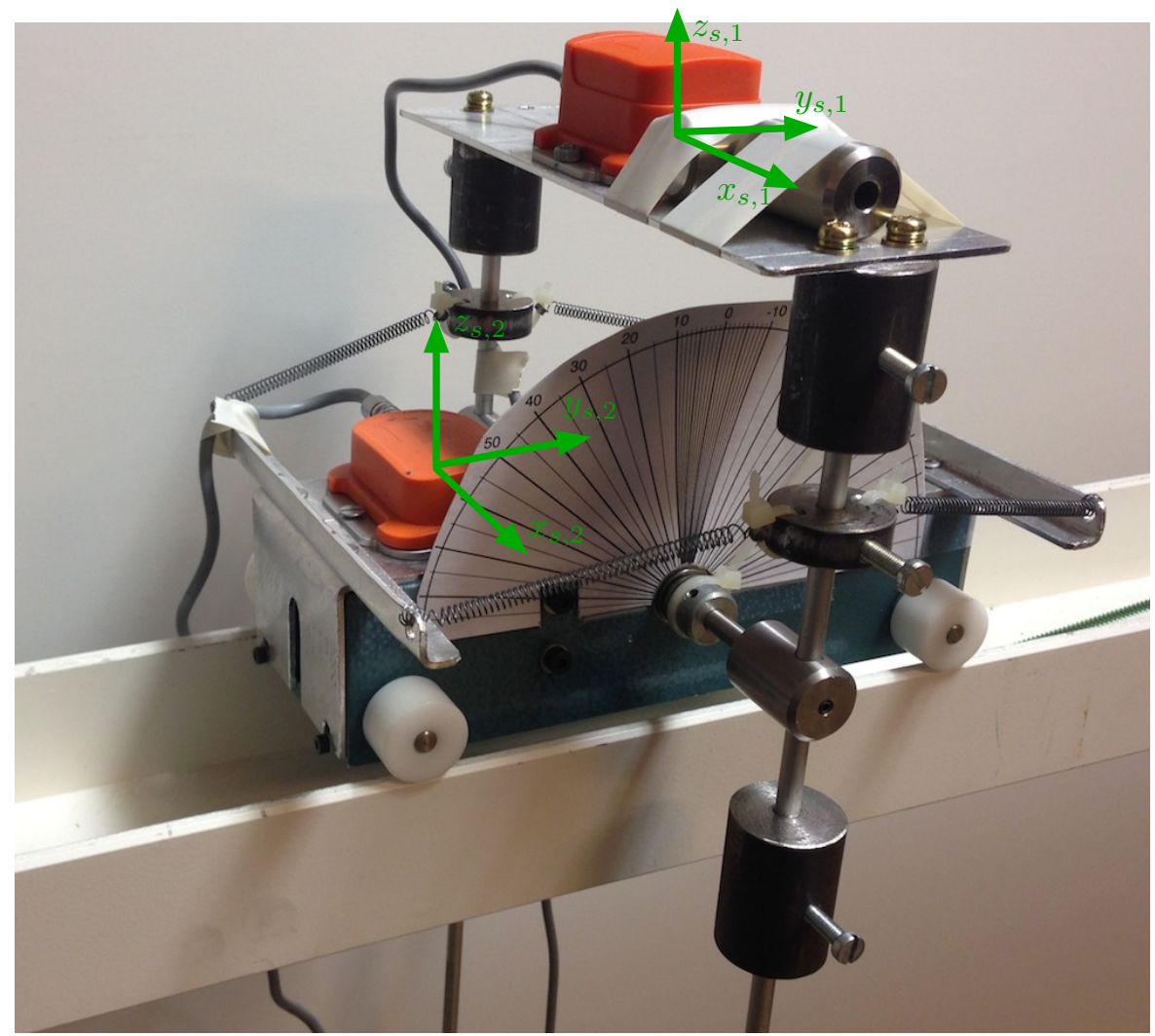

Figure 4.14: A picture of the modified inverted pendulum. Uppermost in the picture is the IMU (orange) measuring the pendulum motion. In front of it is the additional load fastened with white tape. To the left on the cart is the reference IMU. 
Table 4.3: The physical quantities of the system measured or calculated from known physical quantities.

\begin{tabular}{cccc}
\hline Inertia $\left(I_{x}\right)$ & Mass $(M)$ & CM $\left(z_{g}\right)$ & IMU position $\left(z_{s}\right)$ \\
\hline $0.00707 \mathrm{kgm}^{2}$ & $1.324 \mathrm{~kg}$ & $-0.0211 \mathrm{~m}$ & $-0.1163 \mathrm{~m}$ \\
\hline
\end{tabular}

lengths were measured using a scale and slide caliper, respectively. The center of mass was calculated using the known positions and weights of each component. The physical quantities are listed in Table 4.3.

The damping coefficient $d$ was unknown and it was unfortunately difficult to get an independent estimate using the tools that were available at the time of the experiment. The damping torque was due to friction in the bearings of the pendulum rotation axis and the energy dissipated due to the interaction with the data cable attached to the IMU on the pendulum.

The spring coefficient $k$ was also unknown but a rough estimate of the coefficient was found using basic mechanics. The spring torque was created with four linear springs and the elongation of the springs were roughly proportional to the angle of the pendulum. The largest deviation from a pure linear relation between the angle and the elongation was calculated to be $10^{-4} \mathrm{~m}$ up to $20^{\circ}$. This deviation was due the attachment on the pendulum moving in a slight arc. However, simple tests showed that the spring force from each individual spring was not proportional to the elongation. The resulting restoring torque

$$
k(\phi) \phi
$$

was nonlinearly dependent on the angle. Here, $k(\phi)$ is an unknown nonlinear function.

The additional mass was a cylindrical piece of metal seen on the top in Figure 4.14. The known, measured or calculated physical quantities of the additional mass are listed in Table 4.4. The inertia was neglected due to its small size. The second row in Table 4.4 describes the relative difference to the nominal system, for instance, the second column corresponds to $100 \mathrm{~m} / \mathrm{M} \%$. It can be seen that the increase in mass was relatively small, but that the inertia was increased with approximately $20 \%$ and the torque affecting the pendulum was increased roughly 0.2 times for

Table 4.4: The physical quantities of the added mass measured or calculated from known physical quantities. The second row describes the relative difference to the original system

\begin{tabular}{ccccc}
\hline Inertia $\left(I_{x}\right)$ & Mass $(m)$ & $\mathbf{C M}\left(z_{m}\right)$ & $\left(m z_{m}\right)$ & $\left(m z_{m}^{2}\right)$ \\
\hline $5.1 \cdot 10^{-6} \mathrm{kgm}^{2}$ & $0.0975 \mathrm{~kg}$ & $-0.1225 \mathrm{~m}$ & $-0.0119 \mathrm{kgm}$ & $0.0015 \mathrm{kgm}^{2}$ \\
- & $7.37 \%$ & - & $42.38 \%$ & $20.68 \%$ \\
\hline
\end{tabular}




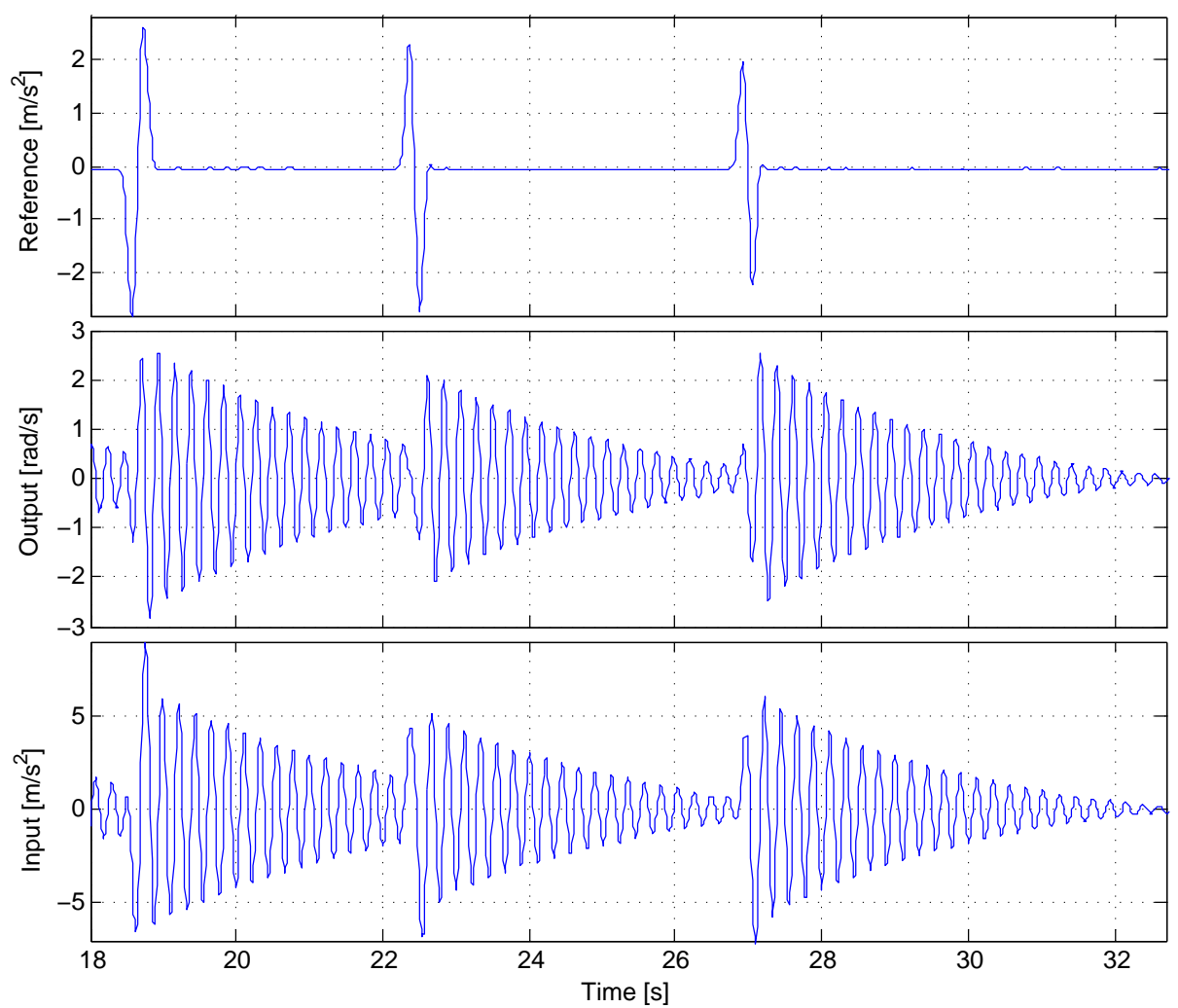

Figure 4.15: A closer look on the nominal dataset.

the same input $a_{y}$ since the coefficient $\beta_{1}$ becomes

$$
\beta_{1}=-\frac{M z_{g}+m z_{m}}{I_{x}+M z_{g}^{2}+m z_{m}^{2}}=4.37
$$

which was a $19.9 \%$ increase from the nominal case.

There were two IMUs mounted on the cart and pendulum as can be seen in Figure 4.14. They were an Xsens MTi (cart) and an Xsens MTi-G (pendulum). Both were equipped with a three-axis accelerometer, a three-axis gyro and a three-axis magnetometer. Only a few of the axes were used to collect data. The $Y$-axis of the accelerometer on the pendulum-IMU corresponds to tangential acceleration (3.44) while the $X$-axis of gyro corresponds to the angular velocity (3.41). The $Y$-axis of the cart-IMU measures the acceleration $y_{4, t}=a_{y, t}+e_{4, t}$, where $e_{4, t}$ was assumed to be discrete-time white zero mean measurement noise, i.e. $F_{r}(q)=1$. Both IMUs were using a sampling rate of $100 \mathrm{~Hz}$. A hammer was used to perform a series of knocks on the rotation axis in the beginning of each measurement series. Synchronization was then achieved by carrying out a correlation analysis on the corresponding acceleration data.

To investigate the necessity of estimating a noise model, two different models were 
tested:

1. The noise model

$$
\bar{H}_{d}(q)=\frac{1+\bar{c}_{1} q^{-1}+\bar{c}_{2} q^{-2}+\bar{c}_{3} q^{-3}+\bar{c}_{4} q^{-4}}{1+\bar{\alpha}_{1} q^{-1}+\bar{\alpha}_{2} q^{-2}},
$$

which corresponds to the prefilter

$$
L(q)=\frac{1}{1+\bar{c}_{1} q^{-1}+\bar{c}_{2} q^{-2}+\bar{c}_{3} q^{-3}+\bar{c}_{4} q^{-4}}
$$

Note that, this noise model was flexible enough to capture $H_{d}(q)$ and also gives some flexibility for coloring in $\bar{\tau}$.

2. The noise model

$$
\bar{H}_{d}(q)=\frac{1}{A(q)},
$$

which corresponds to the prefilter $L(q)=1$, i.e. the same noise model that was used in the previous section. Note that this noise model was not flexible enough to capture even $H_{d}(q)$. It did however result in a less computationally expensive algorithm.

In both cases the instruments were created using the constants $n_{\delta, 0}=4, n_{\delta}=0$, $n_{y}=4$ and $n_{u}=4$.

Two datasets were collected according to Section 4.4, one with nominal weight and one with the additional mass. Both datasets were 10 minutes long, the signals were filtered through an FIR equiripple low-pass filter of order 20 with a cutoff frequency of $0.5 \mathrm{~Hz}$ and were down-sampled to $50 \mathrm{~Hz}$ with a total of 30000 samples in each dataset. The system was excited by dragging the cart back and forth. Figure 4.15 shows a typical part of the nominal dataset.

The result can be seen in the top rows of Table 4.5 and the gray bars of Figure 4.16. Table 4.5 shows the estimated parameters and the relative errors. Note that since $d$ and $k$ are unknown, no relative error is given. The cyan bars of Figure 4.16(a) show the results from Step 2 in Approach 4.2 and Figure 4.16(b) shows the result from Step 3. The figures are created by drawing 100000 samples from $\mathcal{N}\left(\hat{\theta}, \hat{P}_{\theta}\right)$ and passing these through the nonlinear transformation in Step 2a and 3a to the physical parameters. The red lines correspond to the true value and the black lines correspond to the sample mean.

The estimation of the noise model and filtering with $L(\mathrm{q})$ had a significant impact. As a comparison, the bottom rows of Table 4.5 and the cyan bars of Figure 4.16 show the result when the more restrictive noise model of case 2 was used. Even though there was just a small difference in the second step, the estimates in the third step had large errors and the variance was significantly increased, compare for instance, the results shown in the top plot of Figure 4.16(b).

Note that no apparent negative effects can be seen from the nonlinear restoring force (4.56). This was another positive aspect since the restoring force of a ship is nonlinear due to its dependency on the hull form as described in Section 3.2.1. 
Table 4.5: The results from identification of the physical parameters of the system shown in Figure 4.14. The first row in each case of noise model contains the estimated value and the second row contains the relative errors.

\begin{tabular}{cccccccc}
\hline Case & $I_{x}$ & $d$ & $k$ & $m$ & $z_{m}$ & $m z_{m}$ & $m z_{m}^{2}$ \\
\hline \multirow{2}{*}{$(4.57)$} & 0.0071 & 0.0032 & 1.84 & 0.0918 & -0.116 & -0.0126 & 0.0012 \\
& $0.602 \%$ & - & - & $5.81 \%$ & $5.52 \%$ & $10.5 \%$ & $17.6 \%$ \\
\hline \multirow{2}{*}{$(4.59)$} & 0.0073 & 0.0031 & 1.87 & 0.0719 & -0.136 & -0.0098 & 0.0006 \\
& $3.2 \%$ & - & - & $26.2 \%$ & $10.8 \%$ & $17.8 \%$ & $11.3 \%$ \\
\hline
\end{tabular}

\subsubsection{Summary of Experimental Studies}

The two experimental studies presented in this section show the applicability of the method to both simulated and real data. The sensitivity shown in Section 4.5.1 could probably be reduced by the more flexible noise model used in Section 4.5.2. However, the intention of this section was not to do an exhaustive experimental study but rather to present a couple of examples with the goal of understanding the properties of the estimator. In Section 4.5.2, the closed-loop viewpoint of Section 4.3.2 enabled a better choice of instruments with inspiration from previous work in closed-loop identification. The suggested algorithm was applied to a set of data from an experimental setup with good results. The results could probably be improved further by choosing instruments for closed-loop identification with noisy input and output data (EIV). Both studies in this section had a quite low impact from the process disturbance and the real challenge lies in getting consistent estimators in situations with more severe disturbances. 

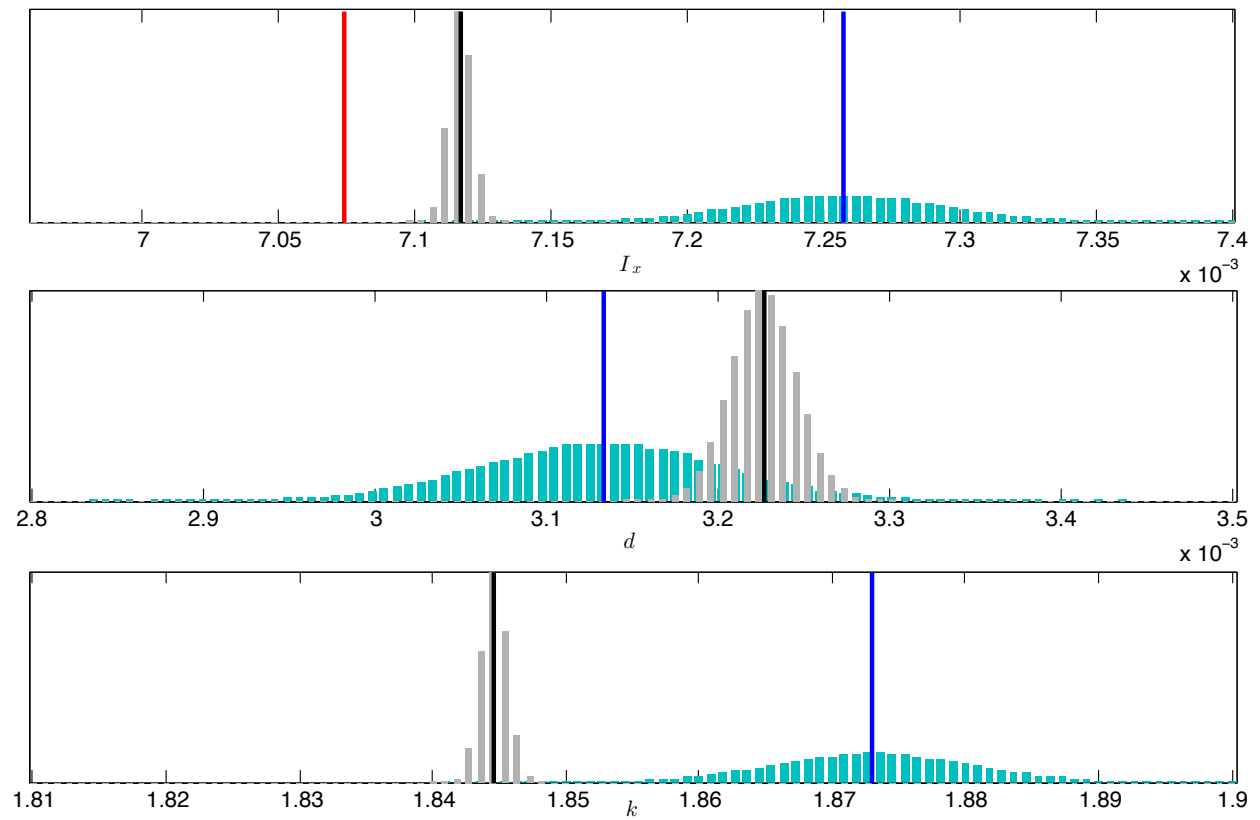

(a) The result from Step 2. Top: $I_{x}$, middle: $d$ and bottom: $k$.
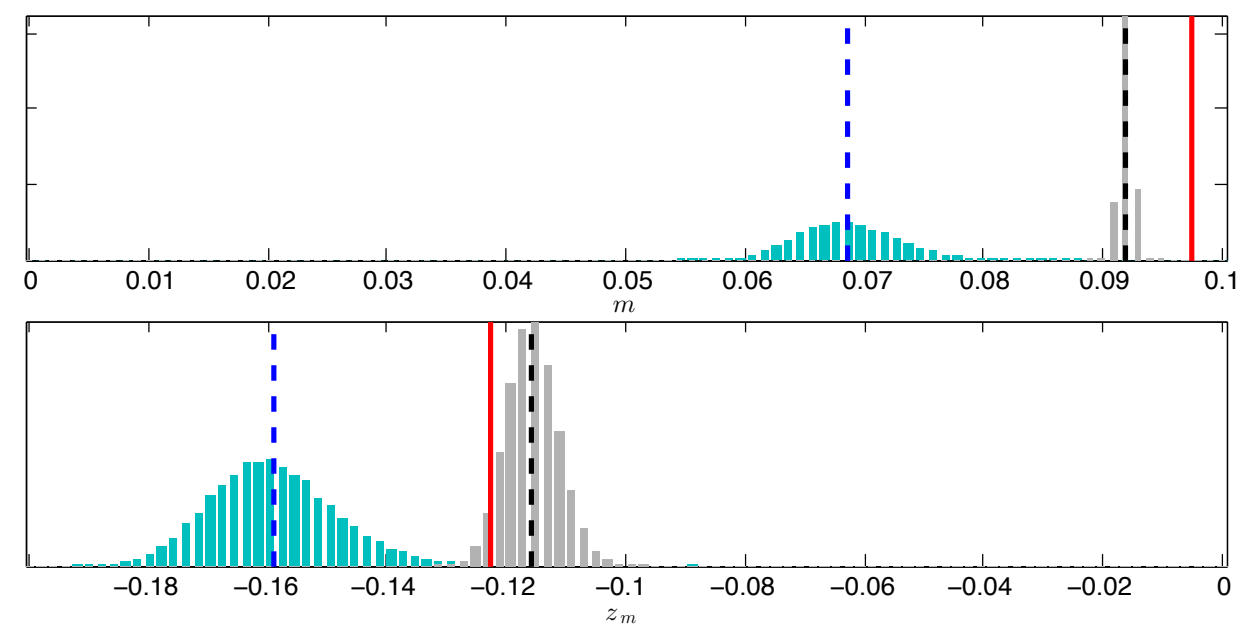

(b) The result from the Step 3. Top: $m$ and bottom: $z_{m}$.

Figure 4.16: Red corresponds to the measured or calculated physical quantity in Table 4.3 and Table 4.4. Gray corresponds to the results from using the noise model $\bar{H}_{d}(q)$ defined in (4.57) and cyan corresponds to the results from using the noise model $\bar{H}_{d}(q)$ defined in (4.59). Blue corresponds to the sample mean for the estimates using the noise model in (4.59). Black corresponds to the sample mean for the estimates using the noise model in (4.57). Note that the IV estimator with the noise model (4.57) has a quite significant decrease in both bias and variance. 


\section{5}

\section{Estimation of a Ship's Roll Dynamics}

This chapter is devoted to graybox modeling of roll dynamics for ships. To avoid a physical model of the entire ship, here, the roll dynamics is seen as a subsystem of the ship's dynamics and the couplings from the other degrees of freedoms are seen as input to the roll subsystem, see Figure 5.1.

To fit into the limited sensor approach for estimation of the parameters from the previous chapter, a linear model of the roll subsystem will be developed from the surge-sway-roll-yaw maneuvering model with rudder input (3.39). Due to identifiability issues, a joint identification approach will be developed. Finally, simulation results showing the applicability of the approach will be presented. For results from this approach on real experimental data from a ship model basin, see Chapter 6.

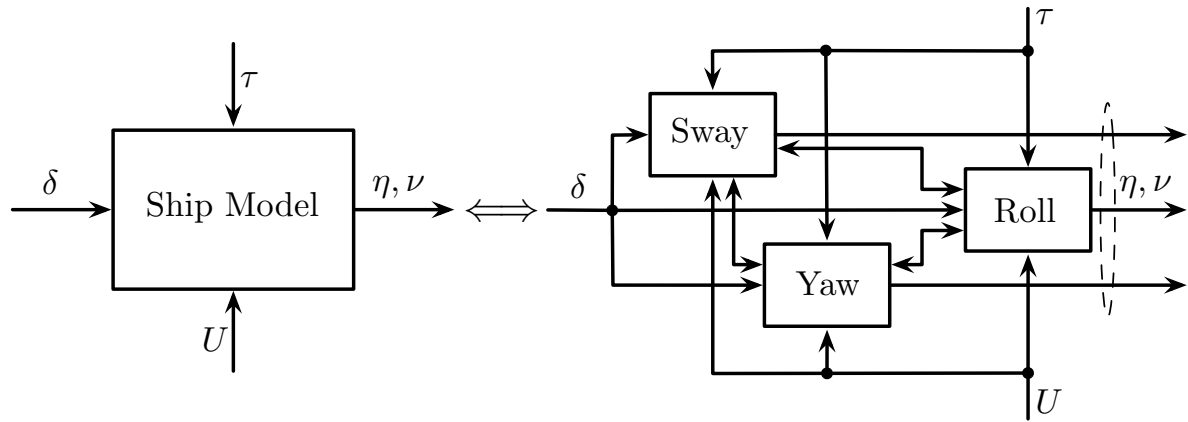

Figure 5.1: The generalized position $\eta$ and generalized speed $\nu$ of a ship model are assumed to be affected by the rudder angle $\delta$, the surge speed $U$ and the process disturbance $\tau$. The roll dynamics is seen as a subsystem of the ship with additional inputs from the sway and yaw subsystems. Note that the surge speed $U$ is here assumed to be decoupled from the other subsystems and that the double-pointed arrows symbolize two-way interaction between the subsystems. 


\subsection{Model Derivation - A Limited Sensor Approach}

The surge-sway-roll-yaw model (3.39) can be written on the nonlinear state-space form

$$
\bar{M} \dot{x}=F(x, \delta)+T \bar{\tau}
$$

where $\bar{\tau}$ is the process disturbance, $T$ is a matrix describing the process disturbance's couplings to the states, $\bar{M}$ is the inertia matrix and $F(x, \delta)$ is the nonlinear state transition function. The states are given by

$$
x=[\phi, \psi, v, p, r]^{T}
$$

where $\phi$ is the roll angle and $\psi$ is the yaw angle, both expressed in the world-fixed coordinate system. The variables $v, p$ and $r$ are the linear surge speed, the roll angular velocity and the yaw angular velocity, respectively, all expressed in the body-fixed frame. For more details on the coordinate systems and notation, see Chapter 3. Finally, $\delta$ is the rudder angle defined in Section 3.3.1.

Assuming that the ship is port-starboard symmetric $\left(\bar{y}_{g}=0\right)$, that the ship is fore-aft symmetric $\left(\bar{x}_{g}=0\right)$ and that the surge component can be decoupled due to much slower dynamics, the linearization around $\bar{x}=0$ and $\bar{\delta}=0$ is

$$
\overline{\boldsymbol{M}} \dot{x}=\left.\frac{\partial F}{\partial x}\right|_{\bar{x}, \bar{\delta}=0} x+\left.\frac{\partial F}{\partial \delta}\right|_{\bar{x}, \bar{\delta}=0} \delta+T \bar{\tau}
$$

where

$$
\begin{aligned}
& \bar{M}=\left[\begin{array}{cc}
I & 0 \\
0 & M
\end{array}\right], \\
& \boldsymbol{M}=\left[\begin{array}{ccc}
M+m-Y_{\dot{v}} & -M z_{g}-m z_{m}-Y_{\dot{p}} & -Y_{\dot{r}} \\
-M z_{g}-m z_{m}-K_{\dot{v}} & \bar{A}_{x} & -K_{\dot{r}} \\
-N_{\dot{v}} & -N_{\dot{p}} & \bar{A}_{z}
\end{array}\right], \\
& \left.\frac{\partial F}{\partial x}\right|_{\bar{x}, \bar{\delta}=0}=\left[\begin{array}{cc:ccc}
0 & 0 & 0 & 1 & 0 \\
0 & 0 & 0 & 0 & 1 \\
\hdashline Y_{\phi u u} U^{2} & 0 & Y_{|u| v}|\bar{U}| & 0 & \left(Y_{u r}-\bar{M}-m \overline{)} \bar{U}\right. \\
K_{\phi u u} U^{2}-\rho g \nabla \overline{G M}_{T} & 0 & K_{|u| v}|U| & K_{p}+K_{|u| p}|U| & \left(K_{u r}+M z_{g}+m z_{m}\right) U \\
N_{\phi u|u|} U|U| & 0 & N_{|u| v}|U| & N_{p}+N_{|u| p}|U| & N_{|u| r}|U|
\end{array}\right]
\end{aligned}
$$

and

$$
\left.\frac{\partial F}{\partial \delta}\right|_{\bar{x}, \bar{\delta}=0}=\left[\begin{array}{c}
0 \\
0 \\
C_{2} \\
-z_{r} C_{2} \\
x_{r} C_{2}
\end{array}\right]
$$

The third row of (5.3) gives a model of the roll subsystem in component form and it is given by

$$
\begin{aligned}
\bar{A}_{x} \dot{p}-\left(K_{\dot{v}}\right. & \left.+M z_{g}+m z_{m}\right) \dot{v}-K_{\dot{r}} \dot{r}=\left(K_{\phi u u} U^{2}-\rho g \nabla \overline{G M}_{T}\right) \phi+K_{|u| v \mid}|U| v \\
& +\left(K_{p}+K_{|u| p}|U|\right) p+\left(K_{u r}+M z_{g}+m z_{m}\right) U r-z_{r} C_{2} \delta+\tau
\end{aligned}
$$


where $\tau$ is the component of $T \bar{\tau}$ that affects the roll subsystem. Here, the influence from $K_{\dot{r}} \dot{r}$ and $K_{|u| v \mid}|U| v$ is assumed to be negligible, i.e. these terms are assumed to be zero. To ease notation, three lumped parameters are introduced. These are

$$
\begin{aligned}
k & =-K_{\phi u u} U^{2}+\rho g \nabla \overline{G M}_{T}-M g z_{g}-m g z_{m}, \\
d & =-K_{p}-K_{|u| p}|U|
\end{aligned}
$$

and

$$
K_{\delta}=-z_{r} C_{2}
$$

Noting that $\dot{\phi}=p$ due to the linearization, i.e. small angle assumption, and using the lumped parameters, the approximate roll dynamics can be written as

$$
\begin{aligned}
A_{1} \ddot{\phi} & =-\left(k+M g z_{g}+m g z_{m}\right) \phi-d \dot{\phi} \\
& +\left(K_{\dot{v}}+M z_{g}+m z_{m}\right) \dot{v}+\left(K_{u r}+M z_{g}+m z_{m}\right) U r+K_{\delta} \delta+\tau
\end{aligned}
$$

where

$$
A_{1}=\bar{A}_{x}=A_{x}+M z_{g}^{2}+m z_{m}^{2}=I_{x}-K_{\dot{p}}+M z_{g}^{2}+m z_{m}^{2}
$$

$I_{x}$ is the ship's inertia around the CG, $K_{\dot{p}}$ is the added mass and $\tau$ is the process disturbance. Note that, the relation $\dot{\phi}=p$ is not valid in general, see Section 3.1.2.

Note that (5.9a) equivalently can be written as

$$
K_{\phi u u} U^{2}-\rho g \nabla \overline{G M}_{T}=-k-M g z_{g}-m g z_{m}
$$

which are the restoring coefficients in the models (5.8) and (5.10), respectively. Here, we assume that the parameter $k$ can be seen as representing the physical restoring properties of the ship, for instance, being dependent on factors such as the hull shape, while the term $M g z_{g}+m g z_{m}$ represents the influence by the mass and its location on the restoring properties. The parameter $k$ is thus, given a speed $U$, assumed to be fixed and independent of the loading condition. Hence, it is assumed that a big change in loading condition is captured by $M g z_{g}+m g z_{m}$. Note that $z_{g}$ and $z_{m}$ for a typical surface vessel are negative and hence, increasing the distance from the center of rotation or increasing the mass would result in $-k-M g z_{g}-m g z_{m}$ being positive which would result in the vessel being unstable. See Section 3.2.1 for a definition and discussion of ship stability. The parameter $d$ is in the same way, given a fixed speed $U$, assumed to be constant.

Disregarding that the model (5.10) is a model of a ship subsystem, given $\dot{v}, r$, $\delta$ and $\tau$, the model (5.10) is a second order system similar to the approximate model described in Chapter 4 with the addition of the ship related parameters, $K_{\dot{p}}, K_{\dot{v}}, K_{u r}$ and $K_{\delta}$, corresponding to the hydrodynamic derivatives and direct terms from the rudder. The model (5.10) has the same issues as the model (4.10) of Section 4.2 since $\dot{v}$ is unknown. Again, the approaches enumerated in Section 4.3 can be used to deal with this issue. Since neither $\dot{v}$ nor a model of it is known, an alternative model can be formed by eliminating $\dot{v}$. The key to this elimination is the known relation between the measured tangential acceleration $a_{s}$ defined in (3.43), the acceleration $a_{y}$ and the variable $\dot{v}$. 


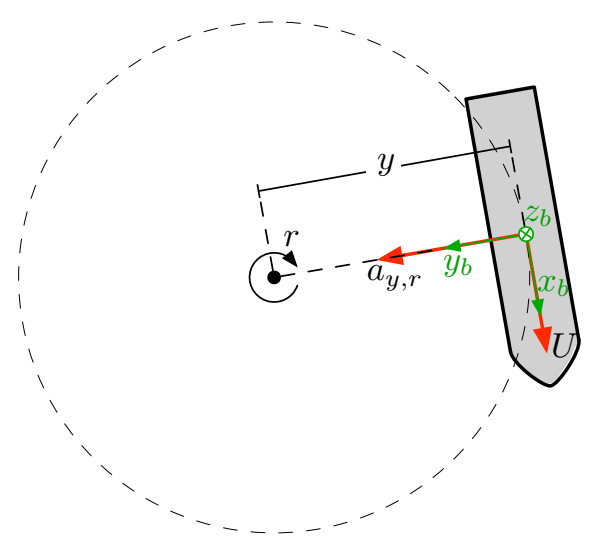

Figure 5.2: A ship moving with constant forward speed on a circle without sideslip has tangential acceleration $U r$ pointing inwards.

The acceleration $a_{y}$ of the ship in the world-fixed $x-y$-plane has two parts. The first contribution emanates from the sway motion and the second part is a contribution due to the angular velocity around the yaw axis. Figure 5.2 shows a ship moving on a circle with the forward speed $U$ and turning with the angular velocity $r$. The resulting acceleration can for instance be derived using the well-known equations for circular motion

$$
\left.\begin{array}{c}
U=y r \\
a_{y, r}=y r^{2}
\end{array}\right\} \Rightarrow a_{y, r}=U r
$$

Furthermore, a ship in motion usually has a discrepancy between the course and the heading due to sideslip, and the sideslip gives the contribution

$$
a_{y, \dot{v}}=\dot{v}
$$

which means that the total sway acceleration is given by

$$
a_{y}=a_{y, \dot{v}}+a_{y, r}=\dot{v}+U r
$$

Hence, the acceleration $\dot{v}$ is measured indirectly by the tangential acceleration $a_{s}$ defined in (3.43) which combined with (5.15) give

$$
a_{s}=z_{s} \ddot{\phi}+g \phi-a_{y}=z_{s} \ddot{\phi}+g \phi-\dot{v}-U r
$$

Solving (5.16) for $\dot{v}$ and substituting it into (5.10) give

where

$$
\begin{aligned}
A_{2} \ddot{\phi}= & -\left(k-K_{\dot{v}} g\right) \phi-d \dot{\phi} \\
& -\left(K_{\dot{v}}+M z_{g}+m z_{m}\right) a_{s}+\left(K_{u r}-K_{\dot{v}}\right) U r+K_{\delta} \delta+\tau
\end{aligned}
$$

$$
A_{2}=A_{x}+M z_{g}\left(z_{g}-z_{s}\right)+m z_{m}\left(z_{m}-z_{s}\right)-K_{\dot{v}} z_{s}
$$

Since the surge speed $U$ is assumed to be constant, this model can be further simplified by introducing the lumped parameter

$$
K_{r}=\left(K_{u r}-K_{\dot{v}}\right) U
$$


giving the surge-speed-free model

$$
A_{2} \ddot{\phi}=-\left(k-K_{\dot{v}} g\right) \phi-d \dot{\phi}-\left(K_{\dot{v}}+M z_{g}+m z_{m}\right) a_{s}+K_{r} r+K_{\delta} \delta+\tau
$$

There are a few properties of the suggested model (5.20) worth observing:

1. The parameters $k, d$ and $K_{r}$ all are dependent on the surge speed $U$ but the dependency is not written out to ease notation.

2. If $K_{u r}=K_{\dot{v}}$, then $r$ is not needed as an input since $a_{s}$ contains all relevant information. This can also be seen in the model (5.10), where the coefficients of $\dot{v}$ and $U r$ would coincide if $K_{u r}=K_{\dot{v}}$.

3. The model (4.21) described in Section 4.3 is obtained if the parameters related to the ship, $K_{\dot{p}}, K_{\dot{v}}, K_{r}$ and $K_{\delta}$, are set to zero.

4. The surge speed $U$ is assumed to be constant. If the speed is measured, it can be used in the identification process either as an input, a measurement or for some linear parameter varying (LPV) type model.

\subsubsection{Center of Rotation}

In Chapter 4 , the $x$-axis of the $b$-frame coincided with the rotation axis. This is not necessarily true in this chapter and the next. Here, the $x_{b}$-axis of the $b$-frame is assumed to be parallel to the rotation axis but shifted in the $z_{b}$-direction, i.e.

$$
z_{g}=\bar{z}_{g}+z_{f}, \quad z_{m}=\bar{z}_{m}+z_{f}
$$

and this can be illustrated as in Figure 5.3. Note that (5.20) is really expressed in the center of rotation coordinate system and $z_{f}$ will express the body-fixed coordinate system in relation to this. However, note that this will not change the estimation problem significantly and the introduction of the extra parameter $z_{f}$ will be equivalent to working with the body-fixed system as if it coincides with the center of rotation coordinate system. The advantage of this formulation is the possibility to estimate the location of the rotation center in relation to the known body-fixed coordinate system, i.e. to estimate $z_{f}$ when the true center of rotation is not known.

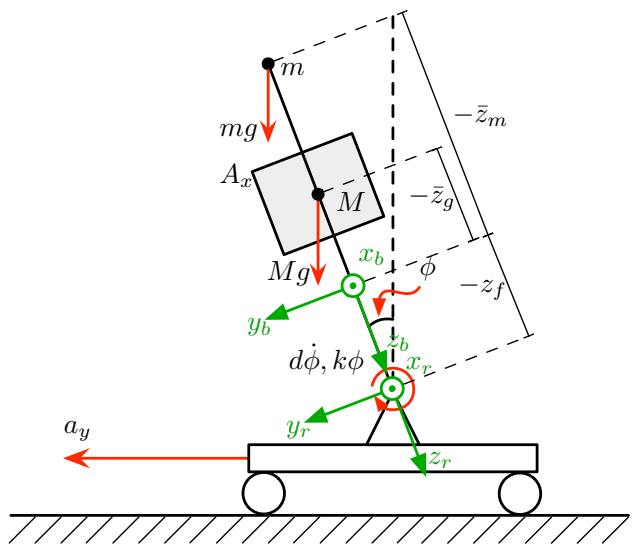

Figure 5.3: $A$ sketch showing the shifted center of rotation in relation to the body-fixed system. 


\subsection{Model Analysis}

This section presents the dependencies of the signals in the model (5.20). These dependencies are important to understand in order to make appropriate choices in the identification procedure. The subsystem view presented in Section 5.1 avoids the use of a complete model of the ship, while the use of an acceleration measurement solves the issue of the unknown input $\dot{v}$. This will however introduce new issues and an analysis is presented in this section. In Section 5.2.1, the signals' dependencies on the true input $\delta$ are discussed. The model (5.20) is also shown to share the important non-minimum phase (NMP) property of the complete model. Section 5.2.2 describes the signals' dependencies on the disturbance $\tau$. It is shown that all signals are correlated with the disturbance and that certain properties of these signals are important in the choice of estimation method.

\subsubsection{The Signals' Dependency on the Input $\delta$}

The ship modeled in this chapter is assumed to be affected by two things, firstly the actuator, i.e. the rudder, and secondly, the disturbances acting on the ship. This section presents the roll dynamics behavior due to the rudder. Neglecting the process disturbance $\tau$, the model (5.20) with $y=\dot{\phi}$ as the output can be written on the form

$$
y=G(\mathrm{p})\left(a_{s}+F_{r} r+F_{\delta} \delta\right)
$$

where the transfer functions are given by

$$
G(\mathrm{p})=\frac{\beta_{1} \mathrm{p}}{\mathrm{p}^{2}+\alpha_{1} \mathrm{p}+\alpha_{2}}, \quad F_{\delta}=\frac{\gamma_{1}}{\beta_{1}}, \quad F_{r}=\frac{\kappa_{1}}{\beta_{1}}
$$

and the parameters are given by

$$
\begin{array}{ll}
\alpha_{1}=\frac{d}{A_{2}}, \quad \alpha_{2}=\frac{k-K_{\dot{v}} g}{A_{2}}, & \beta_{1}=-\frac{K_{\dot{v}}+M z_{g}+m z_{m}}{A_{2}}, \\
\gamma_{1}=\frac{K_{\delta}}{A_{2}}=-\frac{z_{r} C_{2}}{A_{2}} \quad \text { and } & \kappa_{1}=\frac{K_{r}}{A_{2}}
\end{array}
$$

where $A_{2}$ is defined in (5.18).

The reference signal $\delta$ is assumed to be the only input acting on the system, which means that both $a_{s}$ and $r$ depends on $\delta$. Similarly to the model (4.10) in Chapter 4, Proposition 4.1 can be applied to the model (5.10). Assuming that $U$ is constant, the model (5.10) is equivalent to the model (5.20) in positive feedback with $F_{y}=\frac{z_{s} \mathrm{p}^{2}+g}{\mathrm{p}}$ and with $-a_{y}$ as external signal with $a_{y}$ given by (5.15). This gives the model

$$
y=G(\mathrm{p})\left(F_{y}(\mathrm{p}) y+\left(F_{r}-U\right) r-\dot{v}+F_{\delta} \delta\right)
$$

and the structure that can be seen in Figure 5.4. Assuming that the transfer operator from $\delta$ to $\dot{v}$ is $G_{\delta \dot{v}}(\mathrm{p})$ and that the transfer operator from $\delta$ to $r$ is $G_{\delta r}(\mathrm{p}),(5.25)$ can be written

$$
y=G(\mathrm{p})\left(F_{y}(\mathrm{p}) y+\left[\left(F_{r}-U\right) G_{\delta r}(\mathrm{p})-G_{\delta \dot{v}}(\mathrm{p})+F_{\delta}\right] \delta\right)
$$




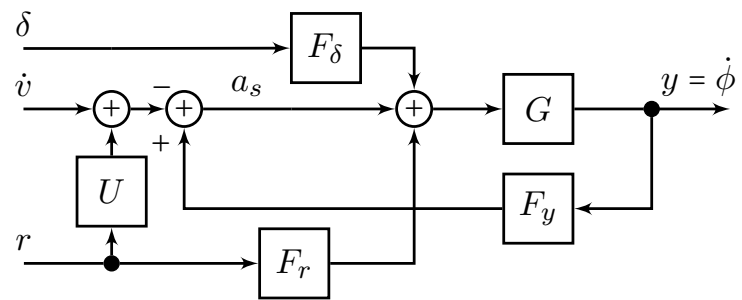

Figure 5.4: As in the model (4.21) case, the model (5.10) is recovered if the acceleration measurement equation (3.43), together with (5.15), is substituted into the model (5.20).

which is equivalent to

$$
y=\frac{G(\mathrm{p})\left[R_{\delta}(\mathrm{p})+F_{\delta}\right]}{1-F_{y}(\mathrm{p}) G(\mathrm{p})} \delta=G_{0}(\mathrm{p})\left[R_{\delta}(\mathrm{p})+F_{\delta}\right] \delta=G_{\delta y}(\mathrm{p}) \delta
$$

where

$$
\begin{gathered}
G_{0}(\mathrm{p})=\frac{G(\mathrm{p})}{1-F_{y}(\mathrm{p}) G(\mathrm{p})}, \\
R_{\delta}(\mathrm{p})=\left(F_{r}-U\right) G_{\delta r}(\mathrm{p})-G_{\delta \dot{v}}(\mathrm{p}), \\
G_{\delta y}(\mathrm{p})=G_{0}(\mathrm{p})\left[R_{\delta}(\mathrm{p})+F_{\delta}\right]
\end{gathered}
$$

and the structure can be seen in Figure 5.5. Hence, (5.30) contains two contributions and these are directly connected to the non-minimum phase behavior described in Section 3.5.1. The contribution $F_{\delta} \delta$ is the torque acting directly on the rotation axis while $R_{\delta}(\mathrm{p}) \delta$ corresponds to the contributions through the slower yaw-dynamics and the hydrodynamic forces. Thus, for a positive rudder angle, the direct term will initially give a negative roll angle that is eventually counteracted by the coupling to the other subsystems, see Section 3.5.1.

In the same way, the transfer function from $\delta$ to $a_{s}$ can be derived from Figure 5.5 and is given by

$$
a_{s}=\frac{F_{y}(\mathrm{p}) G(\mathrm{p})\left(F_{\delta}+F_{r} G_{\delta r}(\mathrm{p})\right)-\left(G_{\delta \dot{v}}(\mathrm{p})-U G_{\delta r}(\mathrm{p})\right)}{1-F_{y}(\mathrm{p}) G(\mathrm{p})} \delta=G_{\delta a_{s}}(\mathrm{p}) \delta
$$

and thus, the non-minimum phase behavior is also visible in the acceleration measurement. But due to the direct terms from $\dot{v}$ and $r$, it gives a more complex response.

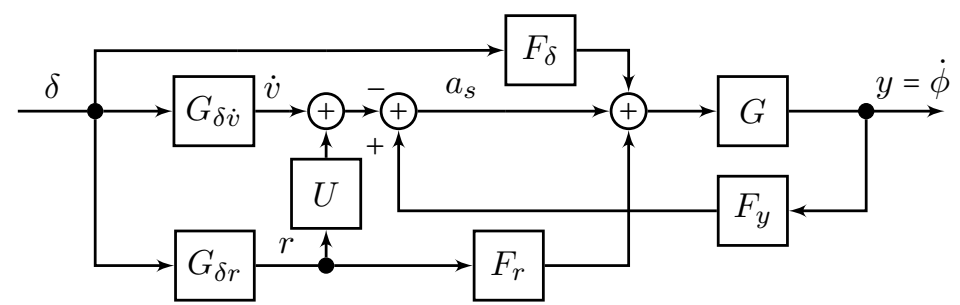

Figure 5.5: The true input to the system is $\delta$ and it also affects $\dot{v}$ and $r$. The dynamics in the transfer functions $G_{\delta \dot{v}}$ and $G_{\delta r}$ together with the direct term in $F_{\delta}$ gives the non-minimum phase behavior of the system. 


\subsubsection{Identification Issues - Correlation with $\tau$}

In the previous section, the signals' dependency of $\delta$ were investigated. From a system identification perspective it is also important to understand the signals' dependency on the process disturbance $\tau$. As mentioned in the previous section, the reference signal $\delta$ is the only input acting on the system, which implies that both $a_{s}$ and $r$ are dependent on $\delta$. However, they are also dependent on $y$ since the modes of the true system are coupled and thus correlated with the process disturbance $\tau$. This means that there are similarities with identification in closedloop and it is important to understand these dependencies to make the correct choices in the identification procedure. To understand the coupling between the subsystems, the model (5.10) can be expressed in terms of the model (5.20) and the subsystems of the linearized system (5.3). This reveals important information about the dependencies between the measured signals.

The model (5.20) in Figure 5.6 with $y=\dot{\phi}$ as the output can be written on the form

$$
y=G(\mathrm{p})\left(a_{s}+F_{r} r+F_{\delta} \delta+\tau\right)
$$

where the transfer functions $G(\mathrm{p}), F_{r}$ and $F_{\delta}$ are defined in (5.23). Assuming that $U$ is constant, the model $(5.10)$ is equivalent to the model (5.20) in positive feedback with $F_{y}=\frac{z_{s} \mathrm{p}^{2}+g}{\mathrm{p}}$ defined in (4.27) and with $-a_{y}$ as external signal with $a_{y}$ given by (5.15). This gives the structure that can be seen in Figure 5.7.

This is however not the whole truth since the process disturbance acting on the roll subsystem will also affect the sway and yaw motions due to the coupling between subsystems. In the same way, disturbances acting directly on the yaw and sway subsystems will propagate to the roll subsystem, see Figure 5.8.

This can be seen by extracting the sway and yaw subsystem from (5.3). Given $r$, $\dot{\phi}$ and $\delta$, the sway subsystem is, after a few manipulations, governed by

$$
\begin{aligned}
& \dot{v}=\frac{\left(M z_{g}+m z_{m}+Y_{\dot{p}}\right) \mathrm{p}^{2}+Y_{\phi u u} U^{2}}{\left(M+m-Y_{\dot{v}}\right) \mathrm{p}-Y_{|u| v}|U|} \dot{\phi} \quad+\frac{\mathrm{p}\left(Y_{\dot{r}} \mathrm{p}+\left(Y_{u r}-M-m\right) U\right)}{\left(M+m-Y_{\dot{v}}\right) \mathrm{p}-Y_{|u| v}|U|} r+\frac{\mathrm{p} C_{2}}{\left(M+m-Y_{\dot{v}}\right) \mathrm{p}-Y_{|u| v \mid}|U|} \delta \\
& =\frac{C}{A} \dot{\phi} \quad+\frac{D}{A} r \quad+\frac{E}{A} \delta
\end{aligned}
$$

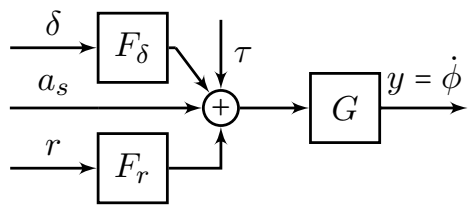

Figure 5.6: The model (5.20) represented in block form. Note that $\delta, a_{s}$ and $r$ are dependent on $y$ according to (5.3). Due to the coupling these signals will also be correlated with $\tau$. 


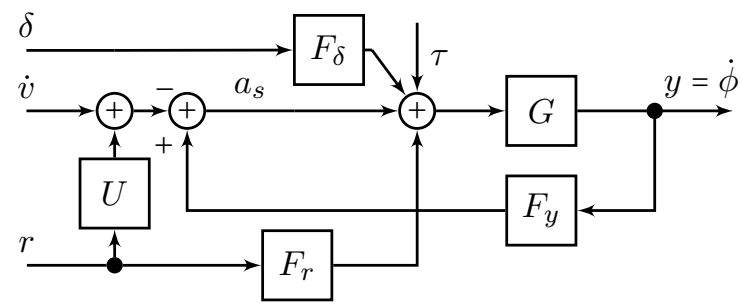

Figure 5.7: As in the model (4.21) case, the model (5.10) is recovered if the acceleration measurement equation (3.43), together with (5.15), is substituted into the model (5.20). This reveals more clearly the issues of using $a_{s}$ as an input to the system, but the complete dependencies between $\dot{v}, r, \dot{\phi}$ and $\delta$ are not shown.

and similarly, given $\dot{v}, \dot{\phi}$ and $\delta$, the yaw subsystem is described by

$$
\begin{aligned}
& r=\frac{N_{\dot{p}} \mathrm{p}^{2}+\left(N_{p}+N_{|u| p}|U|\right) \mathrm{p}+N_{\phi u|u|} U|U|}{\mathrm{p}\left(A_{z} \mathrm{p}-N_{|u| r}|U|\right)} \dot{\phi}+\frac{\left(N_{\dot{v}} \mathrm{p}+N_{|u| v}|U|\right)}{\mathrm{p}\left(A_{z} \mathrm{p}-N_{|u| r}|U|\right)} \dot{v} \quad+\frac{\mathrm{p} x_{r} C_{2}}{\mathrm{p}\left(A_{z} \mathrm{p}-N_{|u| r}|U|\right)} \delta \\
& =\frac{F}{B} \dot{\phi}+\frac{G}{B} \dot{v}+\frac{H}{B} \delta
\end{aligned}
$$

The transfer functions from $\delta$ and $y$ to $\dot{v}$ and $r$ can be obtained by combining (5.33) and (5.34). These transfer functions have the following structures

$$
\dot{v}=\frac{C B+D F}{A B-D G} y+\frac{D H+E B}{A B-D G} \delta \quad \text { and } \quad r=\frac{F A+G C}{A B-D G} y+\frac{G E+H A}{A B-D G} \delta
$$

The dependency of the acceleration $a_{s}$ on $y$ can now be investigated by noting that

$$
a_{s}=\frac{z_{s} \mathrm{p}^{2}+g}{\mathrm{p}} y-\dot{v}-U r=(\overbrace{\frac{z_{s} \mathrm{p}^{2}+g}{\mathrm{p}}-\frac{C B+D F}{A B-D G}-U \frac{F A+G C}{A B-D G}}^{\bar{F}_{y}(\mathrm{p})}) y+\delta \text {-dependent terms }
$$

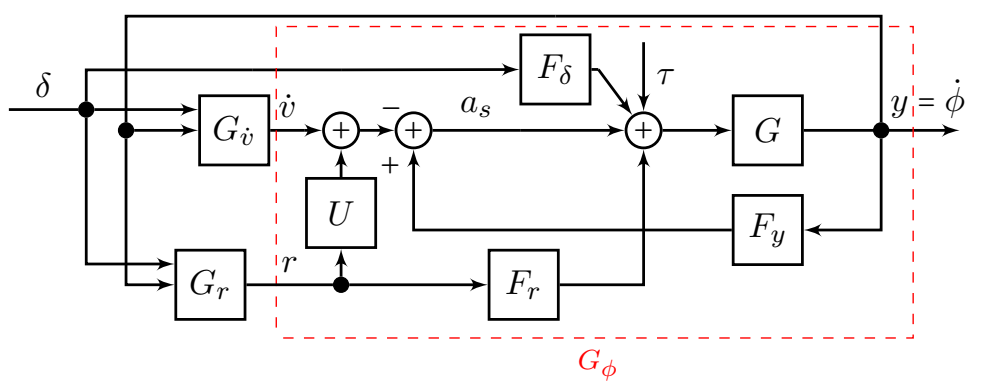

Figure 5.8: The transfer function from $\delta$ to $\dot{\phi}$ viewed in terms of its subsystems. Since the focus is on the roll motion, the emphasis is placed on the model of the roll subsystem $G_{\phi}$ from (5.10) which is shown with its internal dependencies. 
and the loop gain from $\tau$ to $a_{s}$ contains a direct terms since

$$
\begin{aligned}
& \lim _{\mathrm{s} \rightarrow \infty} G(\mathrm{~s}) \bar{F}_{y}(\mathrm{~s})= \\
& \lim _{\mathrm{s} \rightarrow \infty} \frac{\beta_{1} \mathrm{~s}}{\mathrm{~s}^{2}}\left(\frac{z_{s} \mathrm{~s}^{2}}{\mathrm{~s}}-\frac{\left(\left(M z_{g}+m z_{m}+Y_{\dot{p}}\right) \bar{A}_{z}+Y_{\dot{r}} N_{\dot{p}}\right) \mathrm{s}^{4}}{\left(\left(M+m-Y_{\dot{v}}\right) \bar{A}_{z}-Y_{\dot{r}} N_{\dot{v}}\right) \mathrm{s}^{3}}-U \frac{\left(N_{\dot{p}}\left(M+m-Y_{\dot{v}}\right)+\left(M z_{g}+m z_{m}+Y_{\dot{p}}\right)\right) \mathrm{s}^{3}}{\left(\left(M+m-Y_{\dot{v}}\right) \bar{A}_{z}-Y_{\dot{r}} N_{\dot{v}}\right) \mathrm{s}^{3}}\right)= \\
& \lim _{\mathrm{s} \rightarrow \infty} \frac{\beta_{1}}{\mathrm{~s}^{2}}\left(z_{s} \mathrm{~s}^{2}-\frac{\left(M z_{g}+m z m+Y_{\dot{p}}\right) \bar{A}_{z}+Y_{\dot{r}} N_{\dot{p}}}{\left(\left(M+m-Y_{\dot{v}}\right) \bar{A}_{z}-Y_{\dot{r}} N_{\dot{v}}\right)} \mathrm{s}^{2}-U \frac{N_{\dot{p}}\left(M+m-Y_{\dot{v}}\right)+\left(M z_{g}+m z_{m}+Y_{\dot{p}}\right)}{\left(\left(M+m-Y_{\dot{v}}\right) \bar{A}_{z}-Y_{\dot{r}} N_{\dot{v}}\right)} \mathrm{s}\right) \neq 0
\end{aligned}
$$

For the loop gain from $\tau$ to $r$ there is no direct term since

$$
\lim _{\mathrm{s} \rightarrow \infty} G(\mathrm{~s}) F_{r} \frac{F A+G C}{A B-D G}=\lim _{\mathrm{s} \rightarrow \infty} \frac{\beta_{1} \mathrm{~s}}{\mathrm{~s}^{2}} \frac{\left(N_{\dot{p}}\left(M+m-Y_{\dot{v}}\right)+\left(M z_{g}+m z_{m}+Y_{\dot{p}}\right) \mathrm{s}^{3}\right.}{\left(\left(M+m-Y_{\dot{v}}\right) \bar{A}_{z}-Y_{\dot{r}} N_{\dot{v}}\right) \mathrm{s}^{3}}=0
$$

In conclusion, even though the proposed method avoids building a model of the entire ship, it introduces some new challenges:

1. The signals $r, a_{s}$ and $y$ are all correlated with the disturbance $\tau$.

2. The loop gain from $\tau$ to $a_{s}$ contains a direct term which might make the parameter estimator biased.

Finally, in addition to the process disturbance $\tau$ also the measurement noise for each signal has to be considered. If the model (5.20) is used for estimation, the needed signals, $\dot{\phi}, a_{s}$ and $r$, are not known but measured with noise. Hence, the inputs will be noisy and the identification problem will be of errors-in-variables type.

\subsection{Identifiability of the Roll Dynamics Subsystem}

Compared to the model (4.10), the model (5.10) has three more parameters, $K_{\dot{v}}$, $K_{u r}$ and $K_{\delta}$, and two more inputs, $r$ and $\delta$. Hence, the added degree of freedom given by the extra inputs will not make the extra parameters identifiable and if a subset of the parameters is chosen, it is not obvious which combinations of parameters that can be identified simultaneously. In Section 5.3.1 it will be shown that if only a single dataset is used, then only a subset of the unknown parameters can be estimated simultaneously. In Chapter 4, the estimation problem could be solved in a sequential manor, where the nominal data set was used to estimate a first set of parameters and these parameter estimates were used in combination with a second dataset to estimate a second set of parameters. A linear approach could be used since linear constraints were sufficient to obey the original nonlinear parameterization. In contrast to the approach in Chapter 4, the linear sequential method cannot be used in this chapter due to the higher complexity in the relations between the parameters. Instead, the analysis in Section 5.3.2 will show what can be achieved if several datasets are used concurrently. These results will lead to a nonlinear joint method that will be described in Section 5.5.

Note that we will work with the model (5.10) in this section, but that we could as well have worked with the model (5.20), which has the same identifiability properties. 


\subsubsection{Limitations Using a Single Dataset}

Consider the model

$$
\begin{aligned}
A_{1} \ddot{\phi}= & -\left(k+M g z_{g}+m g z_{m}\right) \phi-d \dot{\phi} \\
& +\left(K_{\dot{v}}+M z_{g}+m z_{m}\right) \dot{v}+\left(K_{u r}+M z g+m z_{m}\right) U r+K_{\delta} \delta+\tau
\end{aligned}
$$

defined in (5.10), with the output

$$
y=\left[\dot{\phi}+e_{1}, a_{s}+e_{2}\right]^{T}=\left[\dot{\phi}+e_{1}, z_{s} \ddot{\phi}+g \phi-\dot{v}-U r+e_{2}\right]^{T}
$$

defined by (3.41), (3.44) together with (5.15) and the input

$$
u=[\dot{v}, r, \delta]^{T}
$$

It can be cast into a state-space form by choosing the states $x=[\phi, \dot{\phi}]^{T}$ and inserting (5.10), (5.15) and (5.21) into (3.44). The effects of measurement and process noise are neglected in this section, i.e. $e_{1}=e_{2}=\tau=0$. The state-space model is

$$
\begin{aligned}
& \dot{x}=\left[\begin{array}{cc}
0 & 1 \\
-\frac{k+M g\left(\bar{z}_{g}+z_{f}\right)+m g\left(\bar{z}_{m}+z_{f}\right)}{A_{1}} & -\frac{d}{A_{1}}
\end{array}\right] x \\
& +\left[\begin{array}{ccc}
0 & 0 & 0 \\
\frac{M\left(\bar{z}_{g}+z_{f}\right)+m\left(\bar{z}_{m}+z_{f}\right)+K_{\dot{v}}}{A_{1}} & \frac{M\left(\bar{z}_{g}+z_{f}\right)+m\left(\bar{z}_{m}+z_{f}\right)+K_{u r}}{A_{1}} U & \frac{K_{\delta}}{A_{1}}
\end{array}\right] u \\
& =A\left(\bar{\vartheta}_{p}\right) x+B\left(\bar{\vartheta}_{p}\right) u \\
& y=\left[\begin{array}{cc}
0 & 1 \\
-\frac{k+M g\left(\bar{z}_{g}+z_{f}\right)+m g\left(\bar{z}_{m}+z_{f}\right)}{\tilde{A}_{1}}+g & -\frac{d}{\tilde{A}_{1}}
\end{array}\right] x \\
& +\left[\begin{array}{ccc}
0 & 0 & 0 \\
\frac{M\left(\bar{z}_{g}+z_{f}\right)+m\left(\bar{z}_{m}+z_{f}\right)+K_{\dot{v}}-\tilde{A}_{1}}{\tilde{A}_{1}} & \frac{M\left(\bar{z}_{g}+z_{f}\right)+m\left(\bar{z}_{m}+z_{f}\right)+K_{u r}-\tilde{A}_{1}}{\tilde{A}_{1}} & \frac{K_{\delta}}{\tilde{A}_{1}}
\end{array}\right] u \\
& =C\left(\bar{\vartheta}_{p}\right) x+D\left(\bar{\vartheta}_{p}\right) u
\end{aligned}
$$

where (5.21) inserted in (5.11) give

$$
\begin{aligned}
& A_{1}=A_{x}+M\left(\bar{z}_{g}+z_{f}\right)^{2}+m\left(\bar{z}_{m}+z_{f}\right)^{2}, \\
& \tilde{A}_{1}=A_{1} /\left(\bar{z}_{s}+z_{f}\right) \text { and } z_{s}=\bar{z}_{s}+z_{f}
\end{aligned}
$$

To investigate the identifiability properties, the state-space model was extended according to (2.39) and the parameters considered unknown are

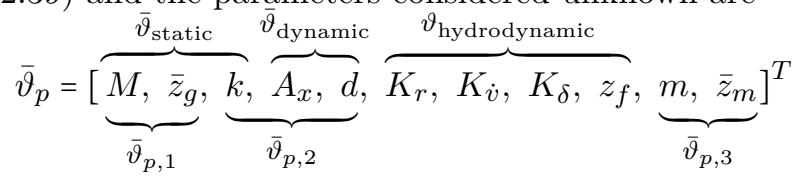

The remaining parameters are assumed to be known. There are $n_{x}=2$ states and $n_{\vartheta}=11$ parameters, i.e. the extended nonlinear system has dimension 13. Since the analysis is performed with a symbolic software, it is assumed that parameters are non-zero and have distinctly different values. It is however worth noting that identifiability might be lost for certain numerical values of the parameters. 
Given that the input $u$ is persistently exciting, the maximum rank of the extended observability matrix $\mathcal{O}$ is 7 and hence, only $7-n_{x}=5$ parameters can be estimated simultaneously. The results of the identifiability analysis are summarized in Table 5.1. The implication of the results is that if the static parameters $\bar{\vartheta}_{\text {static }}$ and the center of rotation in relation to the body-fixed system $z_{f}$ are known, then a sequential method can be used to first estimate the dynamic and hydrodynamic parameters $\vartheta_{\text {hydrodynamic }}$ and $\bar{\vartheta}_{\text {dynamic }}$ using the nominal dataset. The sequential method could still work with linear parameters, but it will require nonlinear constraints to obey the parameterization, which would make the estimation procedure more computationally heavy than the linear approach described in Chapter 4. Furthermore, this would restrict the usability of the algorithm since a lot of information has to be known about the ship. Note that extra sensors are required to estimate $k$ in the first step, for instance, pressure sensors, see Appendix A.

In the subsystem point of view, the input to the roll dynamics subsystem is given by (5.39). However, it is assumed that the true input to the ship is $\delta$ and that this signal will affect all subsystems. The variables $\dot{v}$ and $r$ are thus uniquely determined by $\delta$ and the coupling to the other subsystems similarly to $a_{s}$ in Section 4.3.1. In this section, it is assumed that the other subsystems and the couplings are of sufficient complexity to supply more information than $\delta$ does by itself, i.e. in contrast to $a_{s}$ in Section 4.3.1, $r$ and $\dot{v}$ contains information about the system due to the complexity in the feedback-loops, see (5.35) in Section 5.2.2. For a discussion on complexity and identifiability in closed-loop system identification, see for instance, Gevers et al. (2009) or Bazanella et al. (2010).

\subsubsection{Multi-dataset Identifiability}

To create a system that would also suit a retrofit of older ships, the complexity of the system and the information needed about the ship should be kept to a minimum. Thus, to reduce the complexity of the method it would be preferable if only the parameter $\bar{\vartheta}_{p, 1}$, i.e. the mass $M$ and center of mass $\bar{z}_{g}$ in relation to the body-fixed system, was needed to be known from the first step and that the parameters $\bar{\vartheta}_{p, 2}$ and $\vartheta_{\text {hydrodynamic }}$ could be estimated from nominal data. The only additional knowledge is the position $\bar{z}_{s}$ of the IMU in relation to the body-fixed system.

Table 5.1: The results of the identifiability analysis for the general case of the approximate model. The exceptions are: a) Any three-combination of $\left\{A_{x}, M, \bar{z}_{g}, m, \bar{z}_{m}, z_{f}\right\}$. b) Any combination that contains $\left\{k, K_{r}, K_{\dot{v}}\right\}$ and any two of $\left\{A_{x}, M, \bar{z}_{g}, m, \bar{z}_{m}, z_{f}\right\}$.

\begin{tabular}{cccc}
\hline Case & \# Parameters & Identifiable & Exceptions \\
\hline \multirow{4}{*}{$\delta \neq 0$} & 1 & Yes & - \\
& 2 & Yes & - \\
& 3 & Yes & $\mathrm{a}$ \\
& 4 & Yes & $\mathrm{a}$ \\
& 5 & Yes & $\mathrm{a}, \mathrm{b}$ \\
\hline
\end{tabular}


However, due to identifiability issues this is not possible if only the nominal dataset is used as illustrated in Section 5.3.1. The loaded dataset certainly also contains information about the unknown parameters and hence, the question is if there is enough information contained in both datasets to estimate all parameters at once in a joint approach?

One way of approaching this question is by introducing an artificial MIMO model where the different channels are only related through the physical parameters. By introducing the states

$$
\bar{x}=\left[\phi^{n}, \dot{\phi}^{n}, \phi^{l}, \dot{\phi}^{l}\right]^{T}
$$

the output

$$
\bar{y}=\left[\begin{array}{ll}
y^{n} & y^{l}
\end{array}\right]^{T}
$$

and the input

$$
\bar{u}=\left[\dot{v}^{n}, r^{n}, \delta^{n}, \dot{v}^{l}, r^{l}, \delta^{l}\right]^{T},
$$

the model can be written in the state-space form

$$
\begin{aligned}
& \dot{\bar{x}}=\left[\begin{array}{cc}
A\left(\bar{\vartheta}_{p}^{n}\right) & 0 \\
0 & A\left(\bar{\vartheta}_{p}^{l}\right)
\end{array}\right] \bar{x}+\left[\begin{array}{cc}
B\left(\bar{\vartheta}_{p}^{n}\right) & 0 \\
0 & B\left(\bar{\vartheta}_{p}^{l}\right)
\end{array}\right] \bar{u} \\
& \bar{y}=\left[\begin{array}{cc}
C\left(\bar{\vartheta}_{p}^{n}\right) & 0 \\
0 & C\left(\bar{\vartheta}_{p}^{l}\right)
\end{array}\right] \bar{x}+\left[\begin{array}{cc}
D\left(\bar{\vartheta}_{p}^{n}\right) & 0 \\
0 & D\left(\bar{\vartheta}_{p}^{l}\right)
\end{array}\right] \bar{u}
\end{aligned}
$$

with

$$
\bar{\vartheta}_{p}^{n}=\left.\bar{\vartheta}_{p}\right|_{m=\bar{z}_{m}=0}, \quad \bar{\vartheta}_{p}^{l}=\bar{\vartheta}_{p}
$$

and where $A(\cdot), B(\cdot), C(\cdot)$ and $D(\cdot)$ are defined in (5.40). Here, $z^{n}$ denotes that a signal $z$ belongs to the nominal dataset and $z^{l}$ denotes that a signal $z$ belongs to the loaded dataset. To understand what combinations of parameter combinations that are possible to estimate, the unknown parameters are still given by

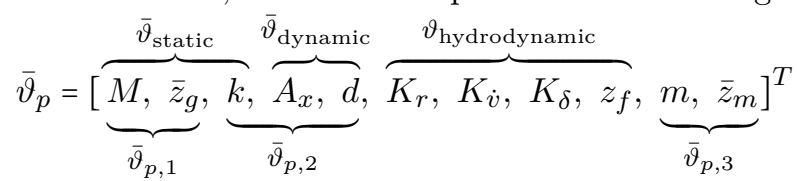

defined in (5.43) and the remaining parameters are assumed to be known. The connection between the channels are thus through the physical parameters.

Assuming that the inputs are persistently exciting, the maximum rank of the extended observability matrix $\mathcal{O}$ for the model (5.47) is 11 . Since there are $n_{x}=4$ states, at most 7 parameters can be estimated simultaneously. It is thus not sufficient to know $\bar{\vartheta}_{p, 1}$, not even if the parameters $\bar{\vartheta}_{\text {static }}$ are assumed to be known, since there are to many unknown parameters to estimate.

One solution is to introduce a second calibration dataset that has a known additional mass $m_{c}$ and center of mass $\bar{z}_{c}$ in relation to the body-fixed coordinate 
system. The set of parameters for this dataset is assumed to be given by

$$
\bar{\vartheta}_{p}^{c}=\left.\bar{\vartheta}_{p}\right|_{m=m_{c}, \bar{z}_{m}=\bar{z}_{c}}
$$

Again, an extended model can be formed by introducing the states

$$
\tilde{x}=\left[\phi^{n}, \dot{\phi}^{n}, \phi^{c}, \dot{\phi}^{c}, \phi^{l}, \dot{\phi}^{l}\right]^{T},
$$

the output

$$
\tilde{y}=\left[y^{n}, y^{c}, y^{l}\right]^{T}
$$

and the input

$$
\tilde{u}=\left[\dot{v}^{n}, r^{n}, \delta^{n}, \dot{v}^{c}, r^{c}, \delta^{c}, \dot{v}^{l}, r^{l}, \delta^{l}\right]^{T},
$$

such that the model can be written in the state-space form as

$$
\begin{aligned}
& \dot{\tilde{x}}=\left[\begin{array}{ccc}
A\left(\bar{\vartheta}_{p}^{n}\right) & 0 & 0 \\
0 & A\left(\bar{\vartheta}_{p}^{c}\right) & 0 \\
0 & 0 & A\left(\bar{\vartheta}_{p}^{l}\right)
\end{array}\right] \bar{x}+\left[\begin{array}{ccc}
B\left(\bar{\vartheta}_{p}^{n}\right) & 0 & 0 \\
0 & B\left(\bar{\vartheta}_{p}^{c}\right) & 0 \\
0 & 0 & B\left(\bar{\vartheta}_{p}^{l}\right)
\end{array}\right] \tilde{u} \\
& \tilde{y}=\left[\begin{array}{ccc}
C\left(\bar{\vartheta}_{p}^{n}\right) & 0 & 0 \\
0 & C\left(\bar{\vartheta}_{p}^{c}\right) & 0 \\
0 & 0 & C\left(\bar{\vartheta}_{p}^{l}\right)
\end{array}\right] \bar{x}+\left[\begin{array}{ccc}
D\left(\bar{\vartheta}_{p}^{n}\right) & 0 & 0 \\
0 & D\left(\bar{\vartheta}_{p}^{c}\right) & 0 \\
0 & 0 & D\left(\bar{\vartheta}_{p}^{l}\right)
\end{array}\right] \tilde{u}
\end{aligned}
$$

where $z^{c}$ denotes that a signal $z$ belongs to the calibration dataset. The maximum rank of the extended observability matrix $\mathcal{O}$ of the model (5.53) is 15 which implies that a maximum of 9 parameters can be simultaneously estimated. All 9 combinations of parameters are identifiable except for any combinations containing all of $\left\{A_{x}, M, \bar{z}_{g}\right\}$ or any combination containing all of $\left\{k, K_{r}, K_{\dot{v}}\right\}$ and any two of $\left\{A_{x}, M, \bar{z}_{g}\right\}$. Most importantly, the desired combination of $\bar{\vartheta}_{p, 2}, \vartheta_{\text {hydrodynamic }}$ and $\bar{\vartheta}_{p, 3}$ is simultaneously identifiable using this approach.

\subsection{Discretization Using Physical Parameters}

As mentioned in the previous section, the connections between the different channels in (5.53) are through the physical parameters. In Chapter 4 it was possible to obey the original nonlinear physical parameterization by linear constraints. In this chapter, this is unfortunately not possible and due to this, a discrete-time model parameterized with the physical parameters is used. The model

$$
A_{2} \ddot{\phi}=-\left(k-K_{\dot{v}} g\right) \phi-d \dot{\phi}-\left(K_{\dot{v}}+M z_{g}+m z_{m}\right) a_{s}+K_{r} r+K_{\delta} \delta+\tau
$$

defined in (5.20) with the output $y=\dot{\phi}$ can be written on the transfer function form

$$
y=G(\mathrm{p})\left(a_{s}+F_{r} r+F_{\delta} \delta+\tau\right)
$$

defined in (5.32) where

$$
G(\mathrm{p})=\frac{\beta_{1} \mathrm{p}}{\mathrm{p}^{2}+\alpha_{1} \mathrm{p}+\alpha_{2}}, \quad F_{\delta}=\frac{\gamma_{1}}{\beta_{1}}, \quad F_{r}=\frac{\kappa_{1}}{\beta_{1}}
$$


are defined in (5.23) and where the parameters

$$
\begin{array}{lll}
\alpha_{1}=\frac{d}{A_{2}}, & \alpha_{2}=\frac{k-K_{\dot{v}} g}{A_{2}}, & \beta_{1}=-\frac{K_{\dot{v}}+M z_{g}+m z_{m}}{A_{2}}, \\
\gamma_{1}=\frac{K_{\delta}}{A_{2}}=-\frac{z_{r} C_{2}}{A_{2}} \quad \text { and } \quad \kappa_{1}=\frac{K_{r}}{A_{2}}
\end{array}
$$

are given by (5.24) with $A_{2}$ defined in (5.18). As in Section 4.3.3, the transfer function $G(\mathrm{p})$ can be discretized using the bilinear transform $\mathrm{p}=\frac{2}{T} \frac{\mathrm{q}-1}{\mathrm{q}+1}$ where $T$ is the sample period. Applying the bilinear transform to (5.24) gives

$$
G_{d}(\mathbf{q})=\frac{\bar{\beta}_{0}\left(1-\mathrm{q}^{-2}\right)}{1+\bar{\alpha}_{1} \mathbf{q}^{-1}+\bar{\alpha}_{2} \mathbf{q}^{-2}}, \quad F_{\delta d}=\frac{\bar{\gamma}_{0}}{\bar{\beta}_{0}}, \quad F_{r d}=\frac{\bar{\kappa}_{0}}{\bar{\beta}_{0}}
$$

with

$$
\bar{\alpha}_{1}=\frac{2 \alpha_{2} T^{2}-8}{\bar{A}_{2}}, \bar{\alpha}_{2}=\frac{4-2 \alpha_{1} T+\alpha_{2} T^{2}}{\bar{A}_{2}}, \bar{\beta}_{0}=\frac{2 \beta_{1} T}{\bar{A}_{2}}, \bar{\kappa}_{0}=\frac{2 \kappa_{1} T}{\bar{A}_{2}}, \bar{\gamma}_{0}=\frac{2 \gamma_{1} T}{\bar{A}_{2}}
$$

and $\bar{A}_{2}=4+2 \alpha_{1} T+\alpha_{2} T^{2}$. By introducing

$$
\mu_{t}^{T}=\left[\mu_{1, t}, \mu_{2, t}, \mu_{3, t}\right]=\left[a_{s, t}-a_{s, t-2}, r_{t}-r_{t-2}, \delta_{t}-\delta_{t-2}\right],
$$

the discrete-time model can be written as the linear regression

$$
y_{t}=\varphi_{t}^{T} \bar{\vartheta}+\tau_{t}
$$

where

$$
\bar{\vartheta}=\left[\bar{\alpha}_{1}, \bar{\alpha}_{2}, \bar{\beta}_{0}, \bar{\kappa}_{0}, \bar{\gamma}_{0}\right]^{T}
$$

and

$$
\varphi_{t}^{T}=\left[-y_{t-1},-y_{t-2}, \mu_{1, t}, \mu_{2, t}, \mu_{3, t}\right]
$$

Note that $\tau_{t}$ is a colored noise process. The discrete-time model can now be directly parameterized by the physical parameters if (5.24) is inserted into (5.55) which gives the nonlinear transformations

$$
g_{\vartheta}\left(\bar{\vartheta}_{p}\right)=\left[\begin{array}{c}
\frac{2\left(k-K_{\dot{v}} g\right) T^{2}-8\left[A_{x}+m z_{m}\left(z_{m}-z_{s}\right)+M z_{g}\left(z_{g}-z_{s}\right)-K_{\dot{v}} z_{s}\right]}{\mathcal{A}_{2}\left(\bar{\vartheta}_{p}\right)} \\
\frac{-2 d T+\left(k-K_{\dot{v}} g\right) T^{2}+4\left[A_{x}+m z_{m}\left(z_{m}-z_{s}\right)+M z_{g}\left(z_{g}-z_{s}\right)-K_{\dot{v}} z_{s}\right]}{\mathcal{A}_{2}\left(\bar{\vartheta}_{p}\right)} \\
-\frac{2 T\left(K_{\dot{v}}+M z_{g}+m z_{m}\right)}{\mathcal{A}_{2}\left(\bar{\vartheta}_{p}\right)} \\
\frac{2 T K_{r}}{\mathcal{A}_{2}\left(\bar{\vartheta}_{p}\right)} \\
\frac{2 T K_{\delta}}{\mathcal{A}_{2}\left(\bar{\vartheta}_{p}\right)}
\end{array}\right]
$$

where

$$
\mathcal{A}_{2}\left(\bar{\vartheta}_{p}\right)=2 d T+\left(k-K_{\dot{v}} g\right) T^{2}+4\left[A_{x}+M z_{g}\left(z_{g}-z_{s}\right)+m z_{m}\left(z_{m}-z_{s}\right)-K_{\dot{v}} z_{s}\right]
$$

The nonlinear transformation (5.60) can be used in estimation to reduce the variance either by directly inserting it into (5.57) or by introducing an equality constraint, i.e. $\bar{\vartheta}-g_{\vartheta}\left(\bar{\vartheta}_{p}\right)=0$, to obey the nonlinear parameterization. 


\subsection{A Joint Identification Approach}

In this section, a joint identification approach is presented and as a first example, the problem solved by the sequential method in Approach 4.1 on page 52 will be expressed in the joint framework. Secondly, estimation of the parameters in the model (5.20) will be discussed using the joint framework and three datasets. In the following chapter, there will be extensive use of one particular version of the joint approach where the estimation steps are computed using the iterative extended IV method described in Section 2.4.2. For this reason, certain aspects of this method will be discussed and the algorithm will be summarized.

\subsubsection{The Pendulum Problem Revisited - A Joint Approach}

In the sequential method described in Approach 4.1, the parameters

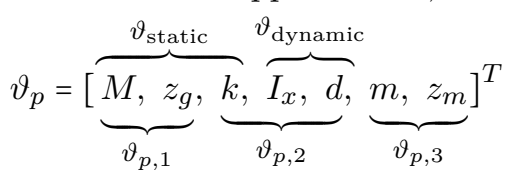

defined in (4.45) are estimated in three steps.

Firstly, the estimate $\hat{\vartheta}_{p, 1}$ is assumed to be known or estimated using a special method. In Step 2, the estimate of $\vartheta_{p, 2}$ is found by computing

$$
\begin{gathered}
\hat{\vartheta}_{p, 2}=\underset{\vartheta_{p, 2}}{\operatorname{argmin}} V_{2}\left(\hat{\vartheta}_{p, 1}, \vartheta_{p, 2}, Z_{n}\right) \\
\text { s.t. } h_{2}\left(\hat{\vartheta}_{p, 1}, \vartheta_{p, 2}\right)=0
\end{gathered}
$$

defined in (4.46) and finally, in Step 3, the estimates $\hat{\vartheta}_{p, 1}$ and $\hat{\vartheta}_{p, 2}$ are used to compute the estimate

$$
\begin{gathered}
\hat{\vartheta}_{p, 3}=\underset{\vartheta_{p, 3}}{\operatorname{argmin}} V_{3}\left(\hat{\vartheta}_{p, 1}, \hat{\vartheta}_{p, 2}, \vartheta_{p, 3}, Z_{l}\right) \\
\text { s.t. } h_{3}\left(\hat{\vartheta}_{p, 1}, \hat{\vartheta}_{p, 2}, \vartheta_{p, 3}\right)=0
\end{gathered}
$$

as defined in (4.47).

In this section we instead solve a joint problem by computing the estimate according to Approach 5.1. In this approach, both datasets are used simultaneously and the parameters $\vartheta_{p, 2}$ and $\vartheta_{p, 3}$ are estimated in one joint estimation problem. One issue with the sequential Approach 4.1 is that the estimate $\hat{\vartheta}_{p, 2}$ is used in Step 3 without considering its uncertainty. The joint approach deals with this automatically at the expense of a nonlinear estimation problem. In Approach 5.1, the criterion function and constraints have deliberately been separated to emphasize the structure in the estimation problem, i.e. the parameters' relation to the different datasets. Note that the datasets do not need to be of equal length for this approach to work and that even several shorter datasets can be incorporated into the same methodology. Also note that the numbering in Approach 5.1 starts at 2 to emphasize that $\vartheta_{p, 1}$ has to be estimated in a step prior to the approach being used. 


\section{Approach 5.1 (Example of the joint identification approach)}

2-3. The estimate $\hat{\vartheta}_{p, 1}$ is used together with the nominal and the loaded datasets to estimate $I_{x}, d, k, m$ and $z_{m}$ by computing

$$
\begin{aligned}
\left\{\hat{\vartheta}_{p, 2}, \hat{\vartheta}_{p, 3}\right\}=\underset{\vartheta_{p, 2}, \vartheta_{p, 3}}{\operatorname{argmin}} & V_{2}\left(\hat{\vartheta}_{p, 1}, \vartheta_{p, 2}, Z_{n}\right)+V_{3}\left(\hat{\vartheta}_{p, 1}, \vartheta_{p, 2}, \vartheta_{p, 3}, Z_{l}\right) \\
& \text { s.t. } \quad h_{2}\left(\hat{\vartheta}_{p, 1}, \vartheta_{p, 2}\right)=0 \\
& h_{3}\left(\hat{\vartheta}_{p, 1}, \vartheta_{p, 2}, \vartheta_{p, 3}\right)=0
\end{aligned}
$$

where $V_{2}(\cdot)$ and $V_{3}(\cdot)$ are nonlinear criterion functions and $h_{2}(\cdot)=0$ and $h_{3}(\cdot)=0$ are possibly nonlinear equality constraints.

\subsubsection{Estimation of the Roll Dynamics Using the Joint Approach}

In a corresponding manner, the estimation of the parameters

$$
\tilde{\vartheta}_{p, 2}=[\underbrace{k, A_{x}, d}_{\bar{\vartheta}_{p, 2}}, \underbrace{K_{r}, K_{\dot{v}}, K_{\delta}, z_{f}}_{\vartheta_{\text {hydrodynamic }}}, \underbrace{m, \bar{z}_{m}}_{\bar{\vartheta}_{p, 3}}]^{T}
$$

of the model (5.20) fits into the joint identification framework.

Firstly, the parameters

$$
\tilde{\vartheta}_{p, 1}=[\underbrace{M, \bar{z}_{g}}_{\bar{\vartheta}_{p, 1}}, m_{c}, \bar{z}_{c}]^{T},
$$

that need to be known a priori are estimated or calculated through some specific experiment. Furthermore, the other parameters, such as, the position $\bar{z}_{s}$ of the IMU and the acceleration of gravity $g$, are assumed to be known. Then, the nominal and calibration datasets

$$
Z_{n}=\left(y_{t}, u_{t}, \delta_{t}\right)_{t=1+t_{n}}^{N_{n}+t_{n}} \text { and } Z_{c}=\left(y_{t}, u_{t}, \delta_{t}\right)_{t=1+t_{c}}^{N_{c}+t_{c}}
$$

are collected. Finally, when a loaded dataset

$$
Z_{l}=\left(y_{t}, u_{t}, \delta_{t}\right)_{t=1+t_{l}}^{N_{l}+t_{l}}
$$

has been collected, the change in mass $m$ and change in center of mass $\bar{z}_{m}$ can be estimated simultaneously with the nominal and hydrodynamic parameters according to Approach 5.2. Here, lower and upper bounds on the parameters are introduced in the form of inequality constraints. These bounds can be used to ensure that the estimated parameters become physically reasonable, for example, that the inertia is positive. The different starting times are marked using $t_{n}, t_{c}$ and $t_{l}$ to emphasize that these datasets are not collected at the same time. As in the previous section, the datasets do not necessarily have to be of the same length and several shorter datasets can be incorporated into the same methodology. 


\section{Approach 5.2 (Example of the joint identification approach)}

The estimate $\hat{\tilde{\vartheta}}_{p, 1}$ is used together with the two nominal datasets, $Z_{n}$ and $Z_{c}$, and the loaded dataset $Z_{l}$ to estimate $\tilde{\vartheta}_{p, 2}$ defined in (5.63) by computing

$$
\begin{array}{cl}
\hat{\tilde{\vartheta}}_{p, 2}=\underset{\tilde{\vartheta}_{p, 2}}{\operatorname{argmin}} & V\left(\hat{\tilde{\vartheta}}_{p, 1}, \tilde{\vartheta}_{p, 2}, Z_{n}, Z_{c}, Z_{l}\right) \\
\text { s.t. } \quad h\left(\hat{\tilde{\vartheta}}_{p, 1}, \tilde{\vartheta}_{p, 2}\right)=0 & \tilde{\vartheta}_{p}^{\text {lb }} \leq \tilde{\vartheta}_{p, 2} \leq \tilde{\vartheta}_{p}^{\mathrm{ub}}
\end{array}
$$

where $V(\cdot)$ is a nonlinear criterion function, $h(\cdot)=0$ is a nonlinear equality constraint and $\tilde{\vartheta}_{p}^{\mathrm{lb}}$ and $\tilde{\vartheta}_{p}^{\mathrm{ub}}$ correspond to the lower and upper bounds, respectively. Here, the inequities should be interpreted as inequalities for each element. Note that $V(\cdot)$ could be a sum of nonlinear functions as in Approach 5.1.

Approach 5.2 can be further specified if the model (5.20) is expressed as a linear regression according to (5.57)-(5.59) and the criterion function is quadratic in the linear parameters. In this case, the criterion function of (5.67) can be written

$$
\tilde{V}\left(\tilde{\vartheta}, \tilde{\vartheta}_{p, 1}, \tilde{\vartheta}_{p, 2}, Z_{n}, Z_{c}, Z_{l}\right)=\sum_{i \in\{n, c, l\}}\left\|\Phi^{i} \bar{\vartheta}_{i}-Y^{i}\right\|_{Q_{i}}^{2}
$$

where $\tilde{\vartheta}=\left[\bar{\vartheta}_{n}^{T}, \bar{\vartheta}_{c}^{T}, \bar{\vartheta}_{l}^{T}\right]^{T}$ are the discrete-time linear parameters defined in (5.58), $\Phi^{i}$ and $Y^{i}$ are given by, for instance, (4.49), and $\|x\|_{Q_{i}}^{2}=x^{T} Q_{i} x, Q_{i} \geq 0, i=\{n, c, l\}$ are weighting matrices. The constraint is given by

$$
\tilde{\vartheta}-\left[g_{\vartheta}^{T}\left(\bar{\vartheta}_{p}^{n}\right), g_{\vartheta}^{T}\left(\bar{\vartheta}_{p}^{c}\right), g_{\vartheta}^{T}\left(\bar{\vartheta}_{p}^{l}\right)\right]^{T}=0
$$

where $g_{\vartheta}\left(\bar{\vartheta}_{p}^{i}\right)$ are the nonlinear transformations defined by (5.60). The discretetime linear parameters can be viewed as auxiliary parameters in the optimization problem and the values of these are not necessarily interesting for the final solution. Note that the variance of the estimator is not increased by these auxiliary variables due to the constraints. These variables might even be helpful when solving the optimization problem since it can be seen as a form of lifting (Albersmeyer and Diehl, 2010).

Here, the discrete-time linear parameters $\bar{\vartheta}_{i}$ are eliminated from the problem by combining (5.68) and (5.69). This give the criterion function

$$
V\left(\tilde{\vartheta}_{p, 1}, \tilde{\vartheta}_{p, 2}, Z_{n}, Z_{c}, Z_{l}\right)=\sum_{i \in\{n, c, l\}}\left\|\Phi^{i} g_{\vartheta}\left(\bar{\vartheta}_{p}^{i}\right)-Y^{i}\right\|_{Q_{i}}^{2}
$$

that is directly parameterized with the physical parameters. Note that $\tilde{\vartheta}_{p, 1}, i=$ $\{n, c, l\}$ and $\tilde{\vartheta}_{p, 2}$ is the union of the parameters in $\bar{\vartheta}_{p}^{i}$. As already mentioned, the estimation problem becomes a nonlinear optimization problem which means that the solution can be sensitive to the initial condition. On the other hand, physical insight can be incorporated into the constraints on the parameters. Due to the 
structure of the problem, the criterion function (5.70) can be written on the form

$$
V\left(\tilde{\vartheta}_{p, 1}, \tilde{\vartheta}_{p, 2}, \tilde{Z}\right)=\left\|\tilde{\Phi} \tilde{\vartheta}\left(\tilde{\vartheta}_{p, 1}, \tilde{\vartheta}_{p, 2}\right)-\tilde{Y}\right\|_{\tilde{Q}}^{2}
$$

by using

$$
\tilde{\vartheta}\left(\tilde{\vartheta}_{p, 1}, \tilde{\vartheta}_{p, 2}\right)=\left[\begin{array}{l}
g_{\vartheta}\left(\bar{\vartheta}_{p}^{n}\right) \\
g_{\vartheta}\left(\bar{\vartheta}_{p}^{c}\right) \\
g_{\vartheta}\left(\bar{\vartheta}_{p}^{l}\right)
\end{array}\right], \tilde{\Phi}=\left[\begin{array}{ccc}
\Phi^{n} & 0 & 0 \\
0 & \Phi^{c} & 0 \\
0 & 0 & \Phi^{l}
\end{array}\right], \tilde{Y}=\left[\begin{array}{c}
Y^{n} \\
Y^{c} \\
Y^{l}
\end{array}\right], \tilde{Q}=\left[\begin{array}{ccc}
Q_{n} & 0 & 0 \\
0 & Q_{c} & 0 \\
0 & 0 & Q_{l}
\end{array}\right]
$$

and where $\tilde{Z}=\left(Z_{n}, Z_{c}, Z_{l}\right)$. This is similar to a model of a MIMO system without coupling between the channels and where the difference is that the datasets could be of different lengths. Since the channels are decoupled, the choices of each individual $\Phi^{i}$ and $Y^{i}$ follow from the discussion in Section 4.4.1. The general approach using (5.70) is described in Approach 5.3.

\section{Approach 5.3 (The joint identification approach)}

The estimate $\hat{\tilde{\vartheta}}_{p, 1}$ defined in (5.64) is used together with the two nominal datasets, $Z_{n}$ and $Z_{c}$, and the loaded datasets $Z_{l}$ to estimate $\tilde{\vartheta}_{p, 2}$ defined in (5.63) by computing

$$
\begin{gathered}
\hat{\tilde{\vartheta}}_{p, 2}=\underset{\tilde{\vartheta}_{p, 2}}{\operatorname{argmin}}\left\|\tilde{\Phi} \vartheta\left(\hat{\tilde{\vartheta}}_{p, 1}, \tilde{\vartheta}_{p, 2}\right)-\tilde{Y}\right\|_{\tilde{Q}}^{2} \\
\text { s.t. } \quad \tilde{\vartheta}_{p}^{\mathrm{lb}} \leq \tilde{\vartheta}_{p, 2} \leq \tilde{\vartheta}_{p}^{\mathrm{ub}}
\end{gathered}
$$

\subsubsection{The Iterative Joint IV Method}

In the next chapter, there will be extensive use of one particular version of Approach 5.3 where (5.73) is computed using the iterative extended instrumental variable (IV) method described in Section 2.4.2. To formalize this method, the creation of instruments for the initialization as well as for the iterative refinement will be discussed. To summarize, the method will finally be given in Algorithm 5.1.

The superscript $i$ is either $n, c$ or $l$ and corresponds to the datasets defined in Section 5.5.2. To ease notation, $i=\{n, c, l\}$ is not explicitly written at all places and if the superscript $i$ is used, it should be understood as for $i=\{n, c, l\}$.

In this section, the estimate (5.73) is computed with the extended IV method which corresponds to choosing

$$
\Phi^{i}=\frac{1}{N_{i}}\left[\zeta_{1}^{i} \cdots \zeta_{N_{i}}^{i}\right]\left[\begin{array}{c}
\left(\bar{\varphi}_{1}^{i}\right)^{T} \\
\vdots \\
\left(\bar{\varphi}_{N_{i}}^{i}\right)^{T}
\end{array}\right] \quad \text { and } \quad Y^{i}=\frac{1}{N_{i}}\left[\begin{array}{lll}
\zeta_{1}^{i} & \cdots & \zeta_{N_{i}}^{i}
\end{array}\right]\left[\begin{array}{c}
\bar{y}_{1}^{i} \\
\vdots \\
\bar{y}_{N_{i}}^{i}
\end{array}\right]
$$

where $\zeta_{t}^{i}$ are the vectors of instruments, $\bar{y}_{t}^{i}=L^{i}(\mathrm{q}) y_{t}^{i}, \bar{\varphi}_{t}^{i}=L^{i}(\mathrm{q}) \varphi_{t}^{i}$ and $L^{i}(\mathrm{q})$ are a stable prefilters. The choices of instruments and prefilters are essential in the IV estimator. Even though any instruments that fulfill the conditions in (2.30) will guarantee consistency, the variance properties of the estimator are greatly affected by the choice of instruments and prefilters. 
Assuming that the models are given on the form

$$
y_{t}^{i}=G_{d}\left(\mathrm{q}, \bar{\vartheta}_{p}^{i}\right)\left(a_{s, t}^{i}+F_{r, d}\left(\bar{\vartheta}_{p}^{i}\right) r_{t}^{i}+F_{\delta, d}\left(\bar{\vartheta}_{p}^{i}\right) \delta_{t}^{i}\right)+H_{0, d}^{i}(\mathrm{q}) e_{t}^{i}=\left(\varphi_{t}^{i}\right)^{T} g_{\vartheta}\left(\bar{\vartheta}_{p}^{i}\right)+\tau_{t}^{i}
$$

where the regression vectors are given by

$$
\varphi_{t}^{i}=\left[-y_{t-1}^{i},-y_{t-2}^{i}, \mu_{1, t}^{i}, \mu_{2, t}^{i}, \mu_{3, t}^{i}\right]^{T},
$$

the parameter vectors are defined in (5.60) and the process disturbances are

$$
\tau_{t}^{i}=A_{d}\left(\mathrm{q}, \bar{\vartheta}_{p}^{i}\right) H_{0, d}^{i}(\mathrm{q}) e_{t}^{i}
$$

where $A_{d}\left(\mathbf{q}, \bar{\vartheta}_{p}^{i}\right)$ are the denominators of $G_{d}\left(\mathbf{q}, \bar{\vartheta}_{p}^{i}\right)$ and it is assumed that $e_{t}^{i}$ are white discrete-time zero-mean noise signals. According to the previous work cited in Section 2.4.2, the optimal prefilters are given by the inverse noise models

$$
L^{i}\left(\mathrm{q}, \bar{\vartheta}_{p}^{i}\right)=\frac{1}{A_{d}\left(\mathrm{q}, \bar{\vartheta}_{p}^{i}\right) H_{0, d}^{i}(\mathrm{q})}
$$

and the optimal instruments are given by the filtered noise-free regression vectors

$$
\zeta_{t}^{i}=L^{i}\left(\mathbf{q}, \bar{\vartheta}_{p}^{i}\right)\left[-\stackrel{\circ}{y}_{t-1}^{i}, \quad-\stackrel{\circ}{y}_{t-2}^{i}, \stackrel{\circ}{\mu}_{1, t}^{i}, \stackrel{\circ}{\mu}_{2, t}^{i}, \mu_{3, t}^{i}\right]^{T}
$$

where $\stackrel{\circ}{z}$ is the noise-free part of the signal $z$. Note that $\mu_{3, t}^{i}=\delta_{t}^{i}-\delta_{t-2}^{i}$ and is thus independent of the process disturbance $\tau$. However, these prefilters and instrument vectors are not implementable in reality since they require the knowledge of the true system and perfect measurements. They can however be used as a guide. Note that the inputs and outputs can be written

$$
\stackrel{\circ}{y}_{t}^{i}=G_{\delta y, d}^{i}\left(\mathbf{q}, \bar{\vartheta}_{p}^{i}\right) \delta_{t}^{i}, \quad \stackrel{\circ}{a}_{s, t}^{i}=G_{\delta a_{s}, d}^{i}\left(\mathbf{q}, \bar{\vartheta}_{p}^{i}\right) \delta_{t}^{i} \quad \text { and } \quad \stackrel{\circ}{r}_{t}^{i}=G_{\delta r, d}^{i}(\mathbf{q}) \delta_{t}^{i}
$$

and if the transfer functions are known, then the signals can be simulated using the external reference $\delta_{t}^{i}$. These transfer functions were derived in Section 5.2.1 and are given by

$$
\begin{aligned}
G_{\delta y, d}^{i}\left(\mathbf{q}, \bar{\vartheta}_{p}^{i}\right)= & G_{0, d}\left(\mathbf{q}, \bar{\vartheta}_{p}^{i}\right)\left[R_{d}^{i}\left(\mathbf{q}, \bar{\vartheta}_{p}^{i}\right)+F_{\delta, d}\left(\bar{\vartheta}_{p}^{i}\right)\right] \\
G_{\delta a_{s}, d}^{i}\left(\mathbf{q}, \bar{\vartheta}_{p}^{i}\right)= & G_{0, d}\left(\mathbf{q}, \bar{\vartheta}_{p}^{i}\right)\left[F_{\delta, d}\left(\bar{\vartheta}_{p}^{i}\right)+F_{r, d}\left(\bar{\vartheta}_{p}^{i}\right) G_{\delta r, d}^{i}(\mathbf{q})\right] \\
& -\frac{G_{\delta \dot{v}, d}^{i}(\mathbf{q})-U G_{\delta r, d}^{i}(\mathbf{q})}{1-F_{y, d}(\mathbf{q}) G_{d}\left(\mathbf{q}, \bar{\vartheta}_{p}^{i}\right)},
\end{aligned}
$$

where

$$
R_{d}^{i}\left(\mathrm{q}, \bar{\vartheta}_{p}^{i}\right)=\left(F_{r, d}\left(\bar{\vartheta}_{p}^{i}\right)-U\right) G_{\delta r, d}^{i}(\mathrm{q})-G_{\delta \dot{v}, d}^{i}(\mathrm{q})
$$

and

$$
G_{0, d}\left(\mathrm{q}, \bar{\vartheta}_{p}^{i}\right)=\frac{G_{d}\left(\mathrm{q}, \bar{\vartheta}_{p}^{i}\right)}{1-G_{d}\left(\mathrm{q}, \bar{\vartheta}_{p}^{i}\right) F_{y, d}(\mathrm{q})}
$$

The method presented in this section is based on the method of Gilson et al. (2006) where the parameters are estimated in an iterative scheme. In each iteration, the vectors of instruments $\zeta_{t}^{i}$ are created by simulating the inputs and the outputs from the external reference by using the latest parameter estimates and the prefilters are updated by estimating a noise model from the residuals. 
In the initializing iteration $\left(0^{\text {th }}\right.$ iteration $)$, the initial instruments are created from data. Firstly, blackbox models of the transfer functions in (5.80) are estimated using the datasets $Z_{i}$. These models are then simulated which gives the signals

$$
\hat{y}_{t}^{i, 0}=\hat{G}_{\delta y, d}^{i, 0}(\mathrm{q}) \delta_{t}^{i}, \quad \hat{a}_{s, t}^{i, 0}=\hat{G}_{\delta a_{s}, d}^{i, 0}(\mathrm{q}) \delta_{t}^{i} \quad \text { and } \quad \hat{r}_{t}^{i, 0}=\hat{G}_{\delta r, d}^{i, 0}(\mathbf{q}) \delta_{t}^{i}
$$

The prefilters are given by

$$
L^{i, 0}\left(\mathrm{q}, \bar{\vartheta}_{p}^{i, 0}\right)=1
$$

and the instrument vectors are created according to

$$
\zeta_{t}^{i, 0}=\left[\hat{y}_{t}^{i, 0} \ldots \hat{y}_{t-n_{y}+1}^{i, 0}, \hat{\mu}_{1, t}^{i, 0} \ldots \hat{\mu}_{1, t-n_{a_{s}+1}}^{i, 0}, \hat{\mu}_{2, t}^{i, 0} \ldots \hat{\mu}_{2, t-n_{r}+1}^{i, 0}, \mu_{3, t}^{i} \ldots \mu_{3, t-n_{\delta}+1}^{i}\right]^{T}
$$

The constants $n_{y}, n_{a_{s}}, n_{r}$ and $n_{\delta}$ are the number of time lags (including the nondelay signal) included in $\zeta_{t}^{i, 0}$ for the output $y$, the acceleration $a_{s}$, the angular velocity $r$ and the rudder angle $\delta$, respectively, i.e. $n_{\delta}=0$ means that $\mu_{3, t}$ is not included in $\zeta_{t}^{i, 0}$.

In the refining iterations ( $j^{\text {th }}$ iteration), firstly, $\hat{\bar{\vartheta}}_{p}^{i, j}$ are estimated using the vectors of instruments and prefilters from the $j-1^{\text {th }}$ iteration. Preferably the transfer functions $G_{\delta y, d}^{i}\left(\mathbf{q}, \bar{\vartheta}_{p}^{i}\right)$ defined in (5.81), $G_{\delta a_{s}, d}^{i}\left(\mathbf{q}, \bar{\vartheta}_{p}^{i}\right)$ defined in (5.82) and $G_{\delta r, d}^{i}(\mathbf{q})$ should be used for simulation. However, $G_{\delta r, d}^{i}(\mathbf{q})$ and $G_{\delta \dot{v}, d}^{i}(\mathrm{q})$ are unknown. Furthermore, $\dot{v}^{i}$ are not measured which means that estimation of the transfer functions $G_{\delta \dot{v}, d}^{i}(\mathrm{q})$ are not possible. Instead the following transfer functions are used

$$
\begin{aligned}
\hat{G}_{\delta y, d}^{i, j}\left(\mathbf{q}, \hat{\bar{\vartheta}}_{p}^{i, j}\right)= & G_{0, d}\left(\mathbf{q}, \hat{\bar{\vartheta}}_{p}^{i, j}\right)\left[\hat{R}_{d}^{i, j}\left(\mathbf{q}, \hat{\bar{\vartheta}}_{p}^{i, j}\right)+F_{\delta, d}\left(\hat{\bar{\vartheta}}_{p}^{i, j}\right)\right] \\
\hat{G}_{\delta a_{s}, d}^{i, j}\left(\mathbf{q}, \hat{\bar{\vartheta}}_{p}^{i, j}\right)= & G_{0, d}\left(\mathbf{q}, \hat{\bar{\vartheta}}_{p}^{i, j}\right)\left[F_{\delta, d}\left(\hat{\bar{\vartheta}}_{p}^{i, j}\right)+F_{r, d}\left(\hat{\bar{\vartheta}}_{p}^{i, j}\right) \hat{G}_{\delta r, d}^{i, 0}(\mathbf{q})\right] \\
& +\frac{U \hat{G}_{\delta r, d}^{i, 0}(\mathbf{q})}{1-F_{y, d}(\mathbf{q}) G_{d}\left(\mathbf{q}, \hat{\bar{\vartheta}}_{p}^{i, j}\right)},
\end{aligned}
$$

where

$$
\hat{R}_{d}^{i, j}\left(\mathrm{q}, \hat{\bar{\vartheta}}_{p}^{i, j}\right)=\left(F_{r, d}\left(\hat{\bar{\vartheta}}_{p}^{i, j}\right)-U\right) \hat{G}_{\delta r, d}^{i, 0}(\mathrm{q})
$$

and $\hat{G}_{\delta r, d}^{i, 0}(\mathrm{q})$ are the blackbox models estimated from data in the initialization step. The transfer functions $(5.88),(5.89)$ and $\hat{G}_{\delta r, d}^{i, 0}(\mathrm{q})$ are then simulated with $\delta_{t}^{i}$ as input, which gives the signals

$$
\hat{y}_{t}^{i, j}=\hat{G}_{\delta y, d}^{i, j}(\mathrm{q}) \delta_{t}^{i}, \quad \hat{a}_{s, t}^{i, j}=\hat{G}_{\delta a_{s}, d}^{i, j}(\mathrm{q}) \delta_{t}^{i} \quad \text { and } \quad \hat{r}_{t}^{i, j}=\hat{G}_{\delta r, d}^{i, 0}(\mathbf{q}) \delta_{t}^{i}
$$

Note that $\hat{r}_{t}^{i, j}=\hat{r}_{t}^{i, 0}, \forall j$ which means that these signals only have to be simulated once. The prefilters are given by

$$
L^{i, j}\left(\mathrm{q}, \bar{\vartheta}_{p}^{i, j}\right)=\frac{1}{\bar{H}_{d}^{i}\left(\mathrm{q}, \hat{\eta}^{i, j}\right)}
$$

where

$$
\bar{H}_{d}^{i}\left(\mathrm{q}, \hat{\eta}^{i, j}\right)
$$

are ARMA models estimated from the residuals

$$
\varepsilon_{t}^{i, j}=y_{t}^{i}-\left(\varphi_{t}^{i}\right)^{T} g_{\vartheta}\left(\hat{\bar{\vartheta}}_{p}^{i, j}\right)
$$


and the instrument vectors are created according to

$$
\begin{aligned}
& \zeta_{t}^{i, j}=L^{i, j}\left(\mathbf{q}, \bar{\vartheta}_{p}^{i, j}\right) \times \\
& {\left[\hat{y}_{t}^{i, j} \ldots \hat{y}_{t-n_{y}+1}^{i, j}, \hat{\mu}_{1, t}^{i, j} \ldots \hat{\mu}_{1, t-n_{a_{s}+1}}^{i, j}, \hat{\mu}_{2, t}^{i, j} \ldots \hat{\mu}_{2, t-n_{r}+1}^{i, j}, \mu_{3, t}^{i} \ldots \mu_{3, t-n_{\delta}+1}^{i}\right]^{T}}
\end{aligned}
$$

The algorithm is summarized in Algorithm 5.1 and will be used for computation of the results in Chapter 6.

\section{Algorithm 5.1 (The iterative joint IV method)}

Here, $z^{i}$ indicates that a signal $z$ belongs to the dataset $i$. The superscript $i$ is either $n, c$ or $l$ and corresponds to the datasets defined in Section 5.5.2. To ease notation, $i=\{n, c, l\}$ is not explicitly written at all places and if the superscript $i$ is used, it should be understood as for $i=\{n, c, l\}$.

(A) Initialize:

(a) Set initial value of $\bar{\vartheta}_{p}^{i, 0}$

(b) Create prefilters $L^{i, 0}\left(\mathrm{q}, \bar{\vartheta}_{p}^{i, 0}\right)$ according to (5.86)

(c) Create initial instruments

i. Estimate blackbox models of the transfer functions $\hat{G}_{\delta y, d}^{i, 0}(\mathrm{q}), \hat{G}_{\delta a_{s}, d}^{i, 0}(\mathrm{q})$ and $\hat{G}_{\delta r, d}^{i, 0}(\mathrm{q})$ in $(5.80)$

ii. Simulate the signals $\hat{y}_{t}^{i, 0}, \hat{a}_{s, t}^{i, 0}$ and $\hat{r}_{t}^{i, 0}$ according to (5.85)

iii. Create the instruments $\zeta_{t}^{i, 0}$ according to (5.87)

(d) Set $j=1$

(B) Estimate parameters:

(a) Compute $\hat{\tilde{\vartheta}}_{p, 2}^{j}$ using (5.73) and (5.74) with $L^{i, j-1}\left(\mathrm{q}, \hat{\bar{\vartheta}}_{p}^{i, j-1}\right)$ and $\zeta_{t}^{i, j-1}$

(b) Estimate the parameters $\eta^{i, j}$ of the models $\bar{H}_{d}^{i}\left(\mathrm{q}, \eta^{i, j}\right)$ from $\varepsilon_{t}^{i, j}=\bar{H}_{d}^{i}\left(\mathrm{q}, \eta^{i, j}\right) e_{t}^{i}$ where $\varepsilon_{t}^{i, j}$ are the residual defined in (5.94)

(c) Create the prefilters $L^{i, j}\left(\mathrm{q}, \hat{\bar{\vartheta}}_{p}^{i, j}\right)$ according to (5.92)

(d) Simulate the signals $\hat{y}_{t}^{i, j}, \hat{a}_{s, t}^{i, j}$ and $\hat{r}_{t}^{i, j}$ according to (5.91)

(e) Create the instruments $\zeta_{t}^{i, j}$ according to (5.95)

(C) Terminate: Increase $j$ and go to Step B while

$$
\left\|\hat{\tilde{\vartheta}}_{p, 2}^{j}-\hat{\tilde{\vartheta}}_{p, 2}^{j-1}\right\|_{2}+\left\|\hat{\eta}^{n, j}-\hat{\eta}^{n, j-1}\right\|_{2}+\left\|\hat{\eta}^{c, j}-\hat{\eta}^{c, j-1}\right\|_{2}+\left\|\hat{\eta}^{l, j}-\hat{\eta}^{l, j-1}\right\|_{2}
$$

is above a threshold or as long as a maximum number of iterations is not reached. Otherwise, return $\hat{\tilde{\vartheta}}_{p, 2}=\hat{\tilde{\vartheta}}_{p, 2}^{j}$ and terminate. 


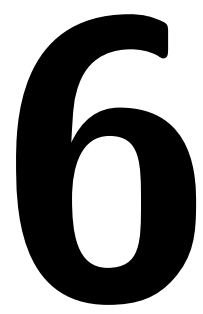

\section{Model Ship Experimental Verification}

This chapter describes an experiment performed to collect data from a scale model of a ship and the estimation results obtained from using these data in the algorithm described in Section 5.5.3. Firstly, the experimental setup will be outlined with emphasis on the system overview. Secondly, the ship will be described in detail, including known physical quantities and calculation of the center of gravity. Thirdly, the experiments will be described and the data will be analyzed. In the last section, estimation results for the input roll subsystem (5.20) using the ship data will be presented.

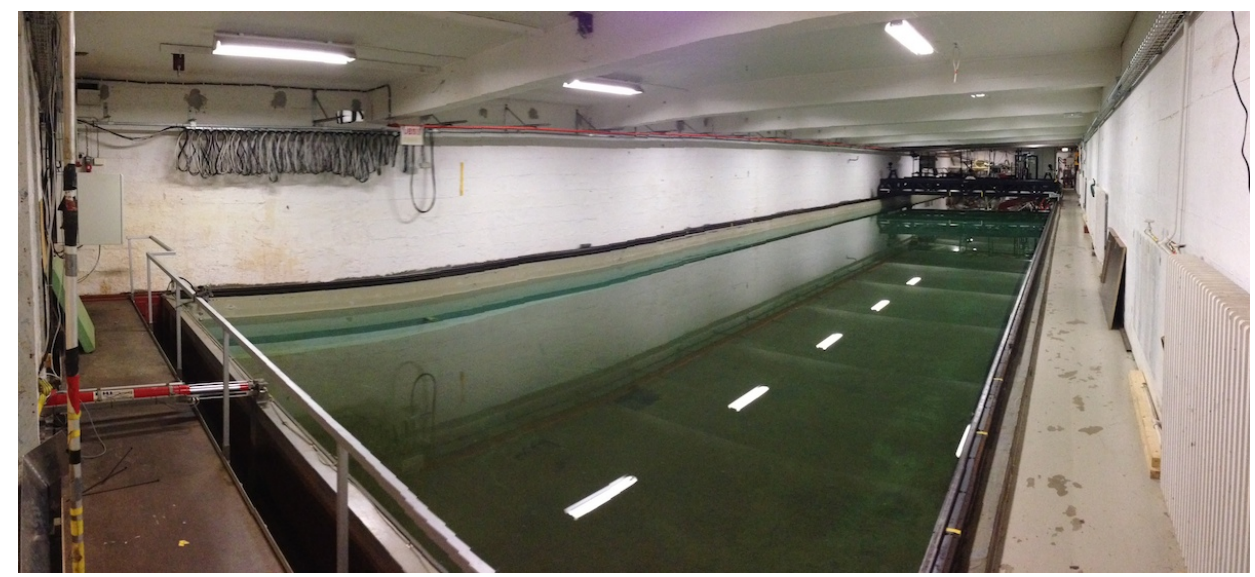

Figure 6.1: An overview of the model basin used in the experiments. The red piston on the left is part of the wave maker, see also Figure 6.2. The three Oqus cameras used for real-time positioning of the ship are mounted on the towing carriage seen on the far right. The towing carriage can be seen in more detail in Figure 6.3. 


\subsection{Hardware - Experiment Overview}

The experiments were performed in the small wave basin Marine Cybernetics Laboratory (MCLab) operated by the Department of Marine Technology at the Norwegian University of Science and Technology (NTNU) in Trondheim. Figure 6.1 shows the basin which is $40 \times 6.45 \times 1.5 \mathrm{~m}$ (length $\times$ width $\times$ depth). The basin had a wave maker that can be seen in the lower left of Figure 6.1 and in the right picture of Figure 6.2. There was also a towing carriage that can be used to drag ships along the basin. In these experiments, the towing carriage was stationary and it was only used for mounting the Oqus cameras used by the Qualisys realtime positioning system. The towing carriage can be seen in Figure 6.1 on the far right and in Figure 6.3.

An overview of the experimental setup can be seen in Figure 6.4. The lines correspond to information flow where the arrows represent the flow direction. The dashed line corresponds to bluetooth communication, dotted lines correspond to WIFI communication and continuous lines correspond to any wired communication or signal. The system had three major components, the ship, the Qualisys realtime positioning system and the human-machine interface. These were basically

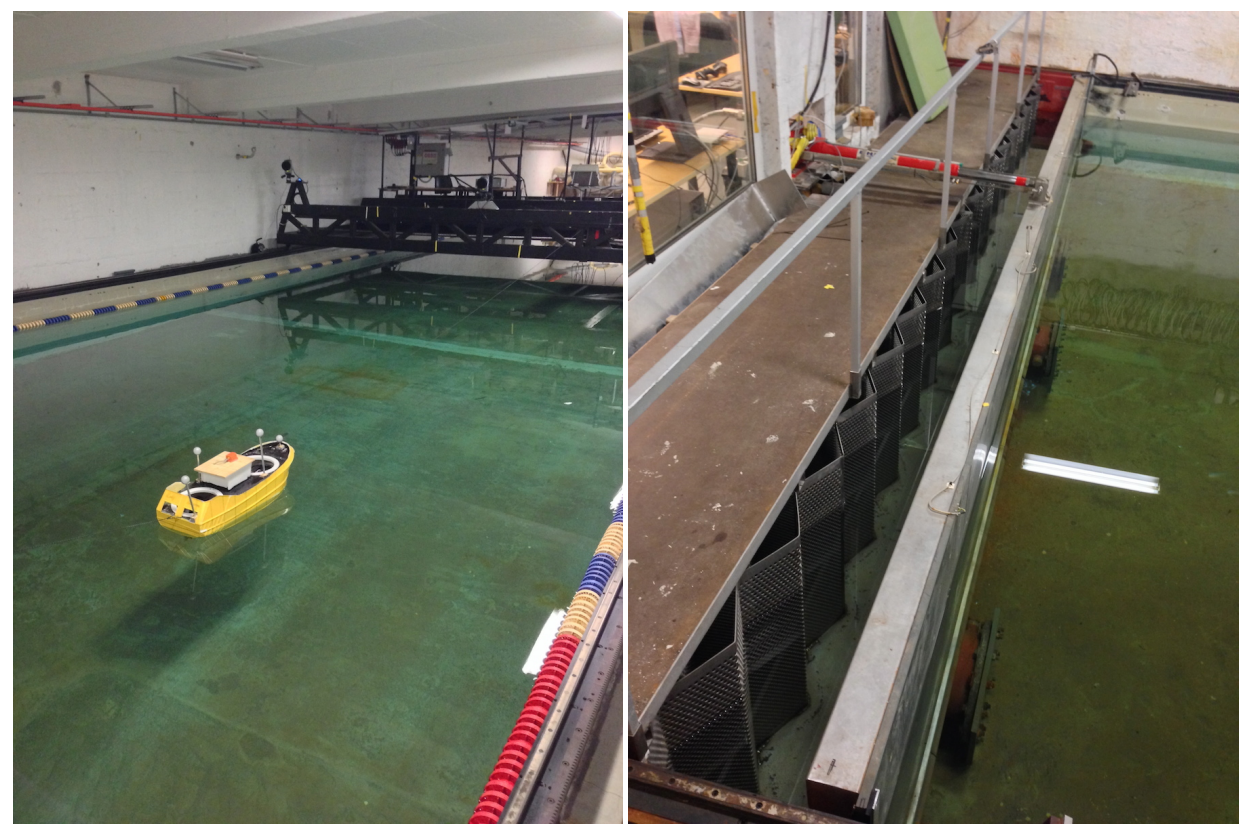

Figure 6.2: Left: An overview of the basin with the model ship to get a sense of scale. Note the IMU on the top of the ship and the markers attached at several points using aluminum tubing. Right: A close up picture of the wave maker seen to the left in Figure 6.1. The wave maker was of single paddle type and was driven by an electrical servo actuator (the red piston) that was controlled by a computer. The synthesized waves could be both regular and irregular. 


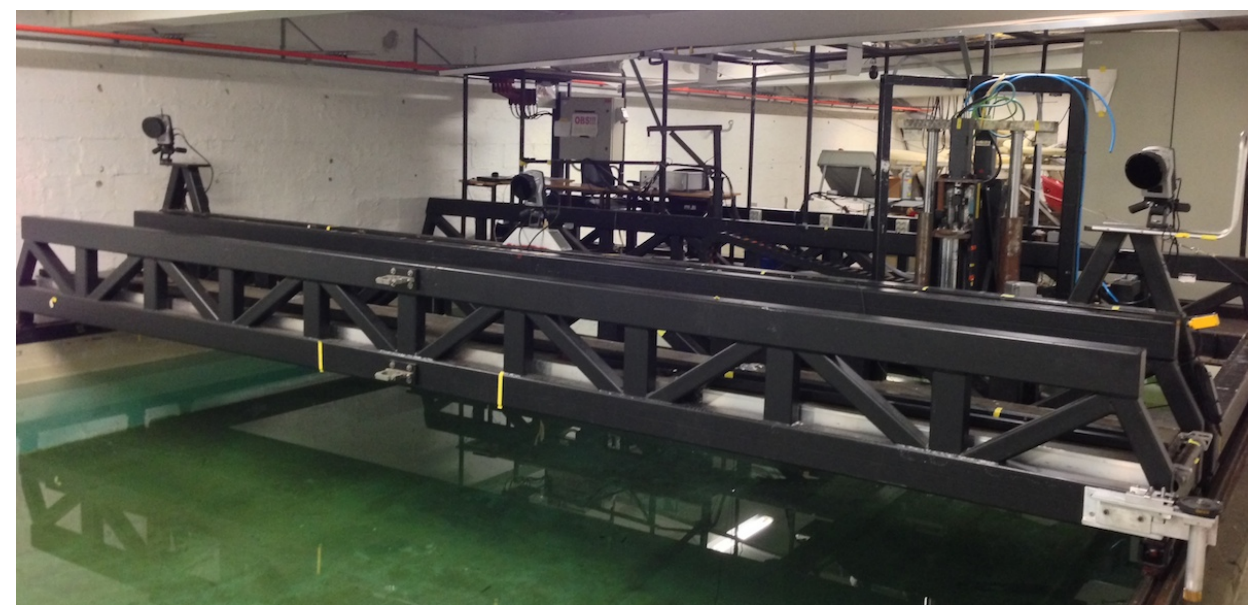

Figure 6.3: A close up of the towing carriage seen on the right in Figure 6.1. In the experiments described in this chapter, the towing carriage was fixed and it was only used for access to the other side of the basin and for mounting the three Oqus cameras used by the Qualisys system. The cameras that were used can be seen on the far left, roughly in the middle and on the right.

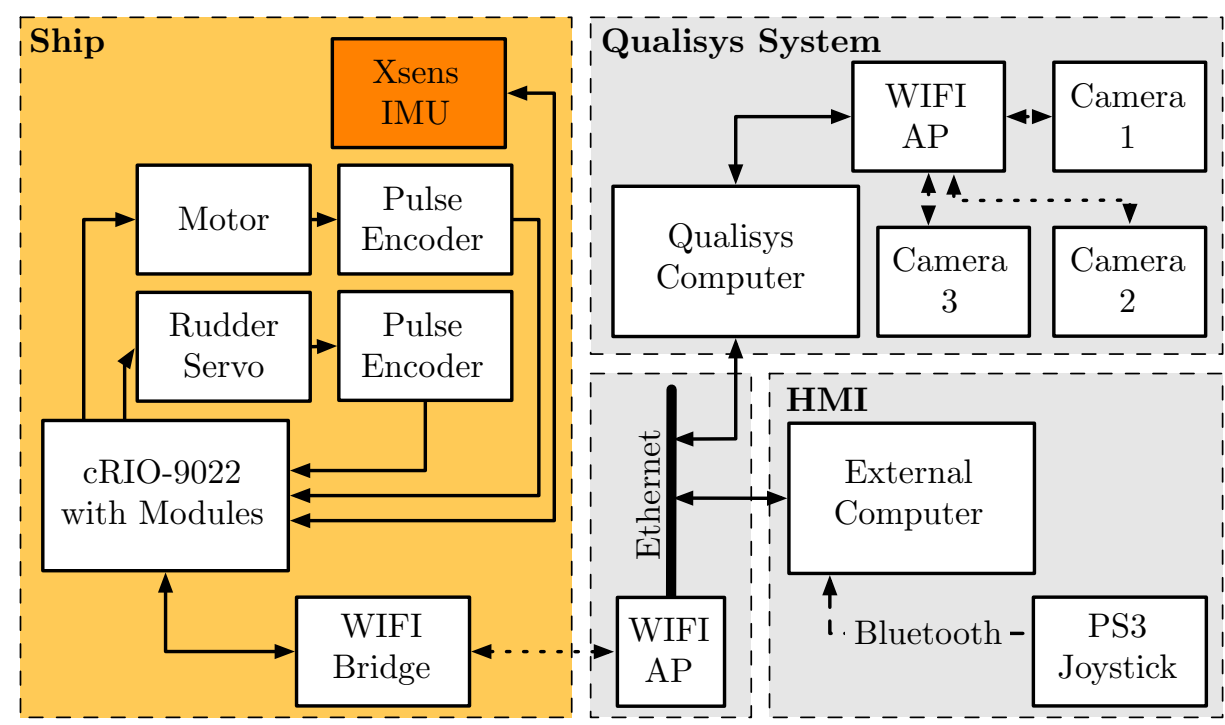

Figure 6.4: An overview of the information flow in the experimental setup. The arrows indicate the flow direction, a dotted line corresponds to WIFI communication and a continuous line corresponds to any wired communication or signal. The system basically consisted of three computers communicating over ethernet and WIFI. The CompactRIO (cRIO) controller on the ship was used to control the motor and rudder, and to collect data from the IMU and the pulse encoders. The Qualisys System was used to track the position and orientation of the ship through the three Oqus cameras and the markers fitted to the ship. The external computer was used to receive input from the user, to display information about the ship and to log data. 
three computers communicating over an ethernet/WIFI network.

In the ship, there was a cRIO Controller that collected data from the IMU and the pulse encoders. The IMU was an Xsens MTi-G that was equipped with a three-axis gyro, a three-axis accelerometer and a three-axis magnetometer, see Section 3.6.1 for more information on the IMU. The cRIO also controled the motor and the rudder of the ship. The controller ran the LabVIEW Real-Time operating system and communicated via a WIFI bridge to the external computer that ran LabVIEW. The external computer was used as a human-machine interface, plotting the ship's current status, logging data and taking commands from a Playstation 3 Joystick. The location of the ship was recorded using a Qualisys system with a computer running the Qualisys Track Manager (QTM) software and three Oqus cameras. This track information was sent to the external computer over ethernet.

The cameras track markers fitted to the ship and the QTM software calculates the position and orientation of the ship in real-time. A schematic view of the wave basin can be seen in Figure 6.5. Four markers had to be seen by at least two cameras for the QTM system to be able to calculate the ship's position and orientation. Due to this there was only a limited usable area that could be used to collect data. Furthermore, for certain positions and orientations, one or two of the markers were hidden behind the ship itself which created blind spots. Several different numbers of markers and positions were tested. The configuration that was chosen and used to collect the data can be seen in Figure 6.6. Since the QTM data was not used in the estimation procedure, this was however not a critical issue. Note that the usable area is larger than what the simplified field of view for the cameras implies.

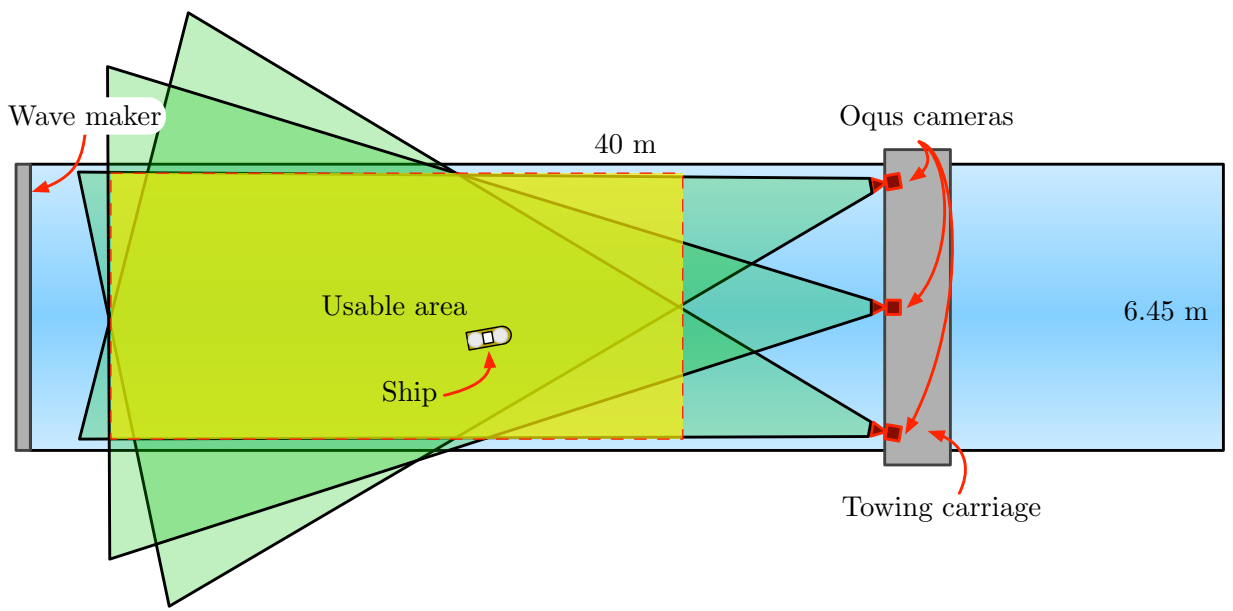

Figure 6.5: A sketch of the model basin seen from the top. The (simplified) fields of view of the cameras are marked in green. There were certain blind spots since enough markers could not be seen by at least 2 cameras at all times. The usable area for collecting data is marked in yellow. Note that the usable area is larger than what the simplified field of view for the cameras implies. 

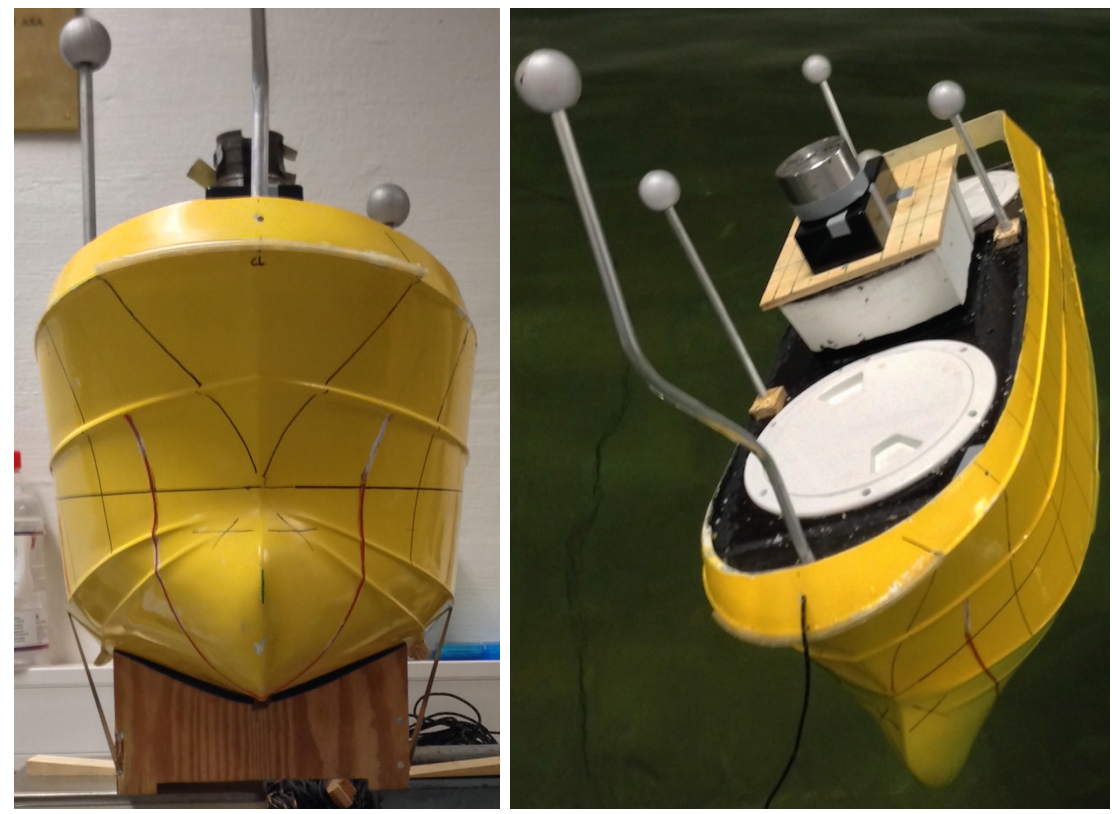

Figure 6.6: Left: The ship seen from the front. Note that the change in wetted surface due to a roll motion should be small since the ship is square in shape. Right: The ship was very sensitive to the loading condition. The picture shows the massive roll angle resulting from an additional mass of $2 \mathrm{~kg}$ corresponding to a $9 \%$ increase in mass. Also note the five markers used by the Qualisys system. The fifth is barely visible behind the black box on the top.

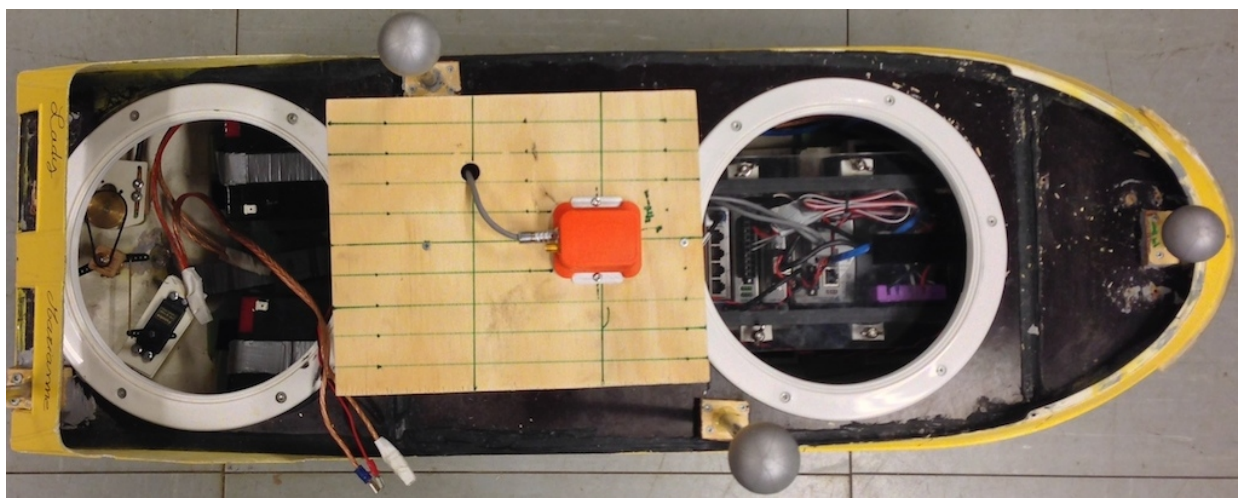

Figure 6.7: The ship seen from the top. To protect the equipment, there were two hatches (white rings) that made the hull water proof. In the aft (left) hatch, the steering servo, the batteries and the pulse encoder measuring the steering angle are visible. In the fore (right) hatch, the CompactRIO-9022 (cRIO) is visible. The IMU (orange) can be seen in the center mounted on top of the bridge. Figure 6.6 shows the ship with the hatches closed and with a black protective box over the IMU. 


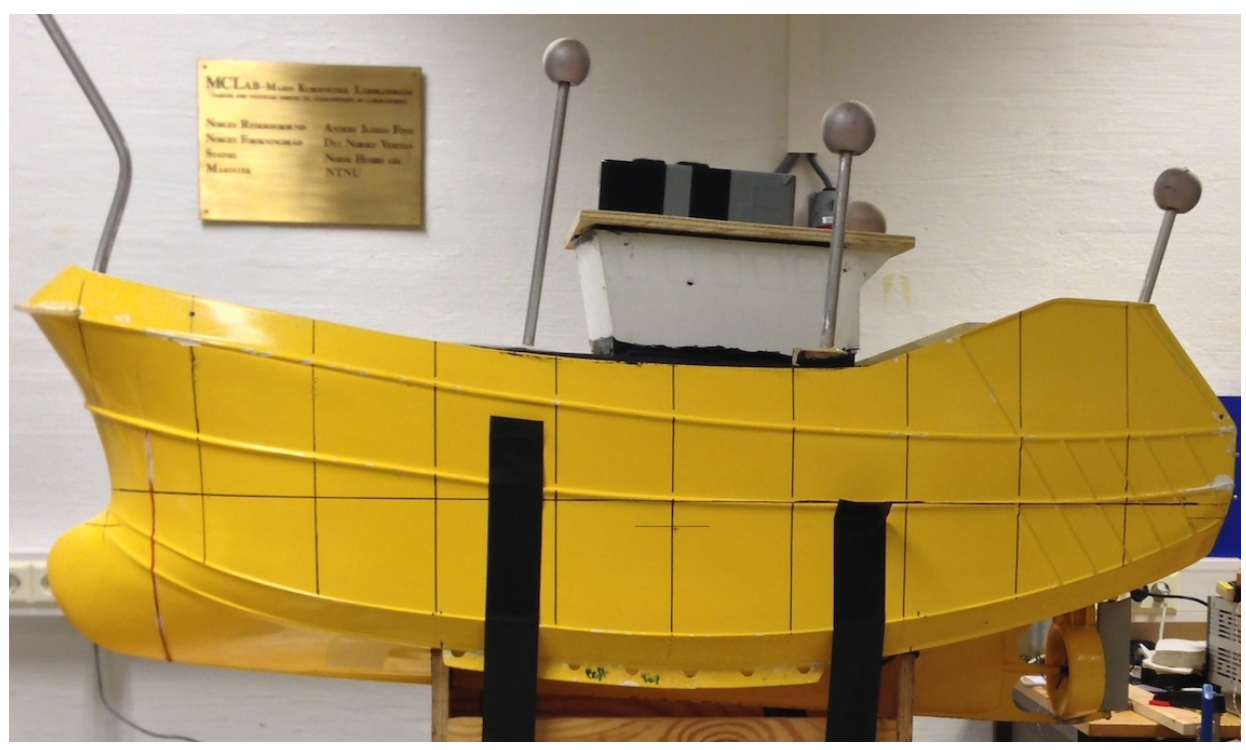

Figure 6.8: A side view of the scale model that was used in the experiments. The IMU was located underneath the black and gray box on the top. Note the only actuators on the ship, the propeller and the rudder, in the lower right corner.

\subsection{Model Ship}

The scale model, hereafter called the ship, was a model of a fishing vessel called "Our Lass II" that is roughly $20 \mathrm{~m}$ long, see Figures 6.2, 6.7, 6.8 and 6.6. Especially note the gray markers used by the Qualisys system. The ship was in scale 1:24 and the known physical quantities are listed in Table 6.1. The ship was made water proof by two hatches seen open in Figure 6.7 as two white rings and closed in Figure 6.6. The ship had its own propulsion and steering that were controlled by the onboard cRIO controller. The propeller and rudder can be seen in the lower right of Figure 6.8. The ship had three onboard sensors, the IMU, measuring angular velocities and linear accelerations, and two pulse encoders that were measuring the RPM of the motor and the steering angle, respectively. In Figure 6.7, the IMU can be seen in the center (orange box), the encoder measuring the steering angle can be seen to the left in the aft hatch (bronze colored wheels) and the cRIO can be seen in the front hatch. The cRIO communicates through a WIFI bridge which was hidden inside the white structure on top of the ship and the cRIO got its input from the Playstation 3 joystick through the external computer. The ship was powered by two batteries that can be seen in the aft hatch of Figure 6.7.

As can be seen in Figures 6.7 and 6.6, the ship is close to square in shape, which meant that for small changes in the roll angle, the wetted surface area should not change much. This implies that the restoring torque will be close to linear for a given forward speed, see Section 3.2.1. 


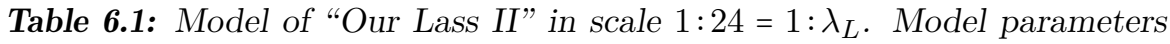
originating from Thys (2013).

\begin{tabular}{lll}
\hline Parameter & Model & Unit \\
\hline Length between perpendiculars $L_{p p}$ & 0.8 & $\mathrm{~m}$ \\
Length at the waterline $L_{w}$ & 0.85 & $\mathrm{~m}$ \\
Beam $B$ & 0.3 & $\mathrm{~m}$ \\
Draft at COG $D$ & 0.144 & $\mathrm{~m}$ \\
Draft fore $D_{f}$ & 0.144 & $\mathrm{~m}$ \\
Draft aft $D_{a}$ & 0.144 & $\mathrm{~m}$ \\
Displacement $\Delta$ & 22.04 & $\mathrm{~kg}$ \\
LCG from midship & 0.125 & $\mathrm{~m}$ \\
$\overline{K G}$ & 0.146 & $\mathrm{~m}$ \\
$\overline{G M}$ & 0.018 & $\mathrm{~m}$ \\
Radius of Gyration in Roll $K_{x x} / B$ & 0.337 & $\mathrm{~m}$ \\
Radius of Gyration in Pitch $K_{y y} / L$ & 0.272 & $\mathrm{~m}$ \\
Radius of Gyration in Yaw $K_{z z} / L$ & 0.272 & $\mathrm{~m}$ \\
\hline
\end{tabular}

The position of the additional mass was chosen to be on top of the black box covering the IMU, see Figure 6.6. Since the distance from the center of rotation $(>20 \mathrm{~cm})$ was large, the weights had quite a big impact on the roll dynamics and stability of the ship. In Figure 6.6, an additional mass of $2 \mathrm{~kg}$ was added to the ship and with this mass it was barely stable, which resulted in a large constant roll angle. Due to this, the additional masses were chosen to be $0.200,0.400$ and $0.600 \mathrm{~kg}$. These correspond to an increase of $0.91 \%, 1.81 \%$ and $2.72 \%$ in the total mass, respectively.

\subsubsection{Calculation of the Ship's Center of Gravity}

As mentioned in Section 5.3, the static parameters $\bar{\vartheta}_{\text {static }}$ or at least the mass $M$ and the center of mass $\bar{z}_{g}$, i.e. $\bar{\vartheta}_{p, 1}$, have to be estimated in a first step. In this experiment, the mass $M$ and the center of mass $\bar{z}_{g}$ were determined by a tilting experiment since the sensors needed for the approach in Appendix A were missing in the scale model.

The tilting experiment was based on the fact that the center of mass was shifting in the $x_{n}$-axis of the world-fixed coordinate system when the ship was tilted an angle $\alpha$. This can be seen in Figure 6.9 where the level (upper) and tilted (lower) case are shown together with the coordinate systems.

The shift in mass was measured using a scale. As can be seen in Figure 6.9, the scale was used to measure the masses $m_{1}$ and $\bar{m}_{1}$ at the forward location and then the masses $m_{2}$ and $\bar{m}_{2}$ were calculated from the total mass $M$. Once these masses were known, the center of mass, i.e. $\bar{x}_{g}$ and $\bar{z}_{g}$, could be calculated using 
the torque equilibrium

$$
0=L_{1} m_{1}+L_{2} m_{2}-m \bar{x}_{m}-M \bar{x}_{g}
$$

in the level case and the torque equilibrium

$$
0=\bar{m}_{1}\left(L_{1} c_{\alpha}-L_{3} s_{\alpha}\right)+\bar{m}_{2}\left(L_{2} c_{\alpha}-L_{3} s_{\alpha}\right)+m\left(\bar{z}_{m} s_{\alpha}-\bar{x}_{m} c_{\alpha}\right)+M\left(\bar{z}_{g} s_{\alpha}-\bar{x}_{g} c_{\alpha}\right)
$$

in the tilted case, by solving for $\bar{x}_{g}$ and $\bar{z}_{g}$. Here, $c_{\alpha}$ and $s_{\alpha}$ means $\cos (\alpha)$ and $\sin (\alpha)$, respectively. To get several independent measurements, the tilting experiment was performed for several different additional masses $m$ and centers of mass $\bar{z}_{m}$, and the result can be seen in Table 6.2. The results show that in average, the center of gravity is $1.8 \mathrm{~mm}$ behind and $7.3 \mathrm{~mm}$ below the reference lines.

\subsection{Experiment Description}

The data was collected in a free run experiments where the ship was untethered and running by its own power. The ship was manually controlled with the Playstation 3 joystick and the rudder angle was thus a true reference to the system that was independent of the other measured signals. Both short and long datasets were collected at constant speed. The shorter sets, one U- or S-turn each, were mainly collected to understand the dynamics and are around $20 \mathrm{~s}$ each. An example of a short dataset can be seen in Figure 6.10. The short test sequences were repeated multiple times for each loading case in Table 6.2. The longer runs did not follow any particular pattern and both short and long turns were performed while utilizing as much of the usable area of the basin as possible. Long datasets were also collected for each loading case in Table 6.2 .

Table 6.2: The results from the center of gravity calculations in the tilting experiment. The parameters $m$ and $\bar{z}_{m}$ refers to the additional mass. The masses $m_{1}$ and $\bar{m}_{1}$ are the measured quantities at the level and the tilted case, respectively. The masses $m_{2}$ and $\bar{m}_{2}$ are calculated as $M-m_{1}$ and $M-\bar{m}_{1}$, respectively. Dash indicates that the quantity is unknown or not applicable.

\begin{tabular}{cccccccc}
\hline \multicolumn{2}{c}{ Load Case } & \multicolumn{2}{c}{ Level } & \multicolumn{2}{c}{ Tilted } & \multicolumn{2}{c}{ Result } \\
\hline$m[\mathrm{~kg}]$ & $\bar{z}_{m}[\mathrm{~m}]$ & $m_{1}[\mathrm{~kg}]$ & $m_{2}[\mathrm{~kg}]$ & $\bar{m}_{1}[\mathrm{~kg}]$ & $\bar{m}_{2}[\mathrm{~kg}]$ & $\bar{x}_{g}[\mathrm{~m}]$ & $\bar{z}_{g}[\mathrm{~m}]$ \\
\hline 0 & - & 11.57 & 11.115 & 14.690 & 7.995 & -0.00178 & 0.00728 \\
0.200 & -0.256 & 11.67 & 11.215 & 14.850 & 7.835 & -0.00181 & 0.00734 \\
0.400 & -0.268 & 11.77 & 11.315 & 15.015 & 7.670 & -0.00185 & 0.00728 \\
0.600 & -0.280 & 11.87 & 11.415 & $>15$ & - & - & - \\
\hline Mean & & & & & & -0.00181 & 0.00730 \\
\hline
\end{tabular}




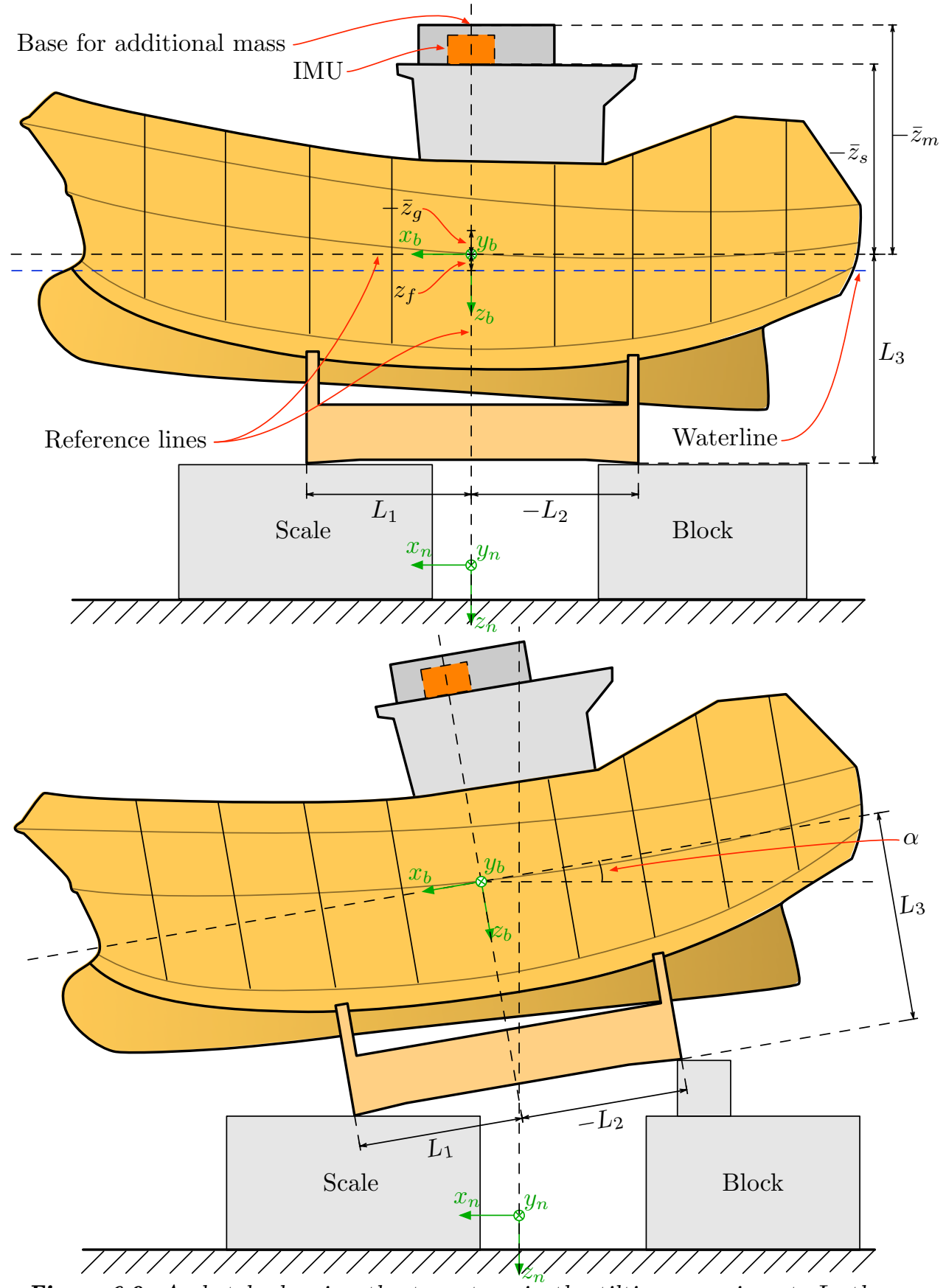

Figure 6.9: $A$ sketch showing the two steps in the tilting experiment. In the top figure, the ship is leveled such that the horizontal reference line (really the reference plane) is orthogonal to the direction of gravity and then the weight was measured using the scale drawn underneath the forward support. The procedure was repeated for the tilted case, except that an angle $\alpha$ was created between the reference line in the level case and the plane corresponding to the tilted case. 


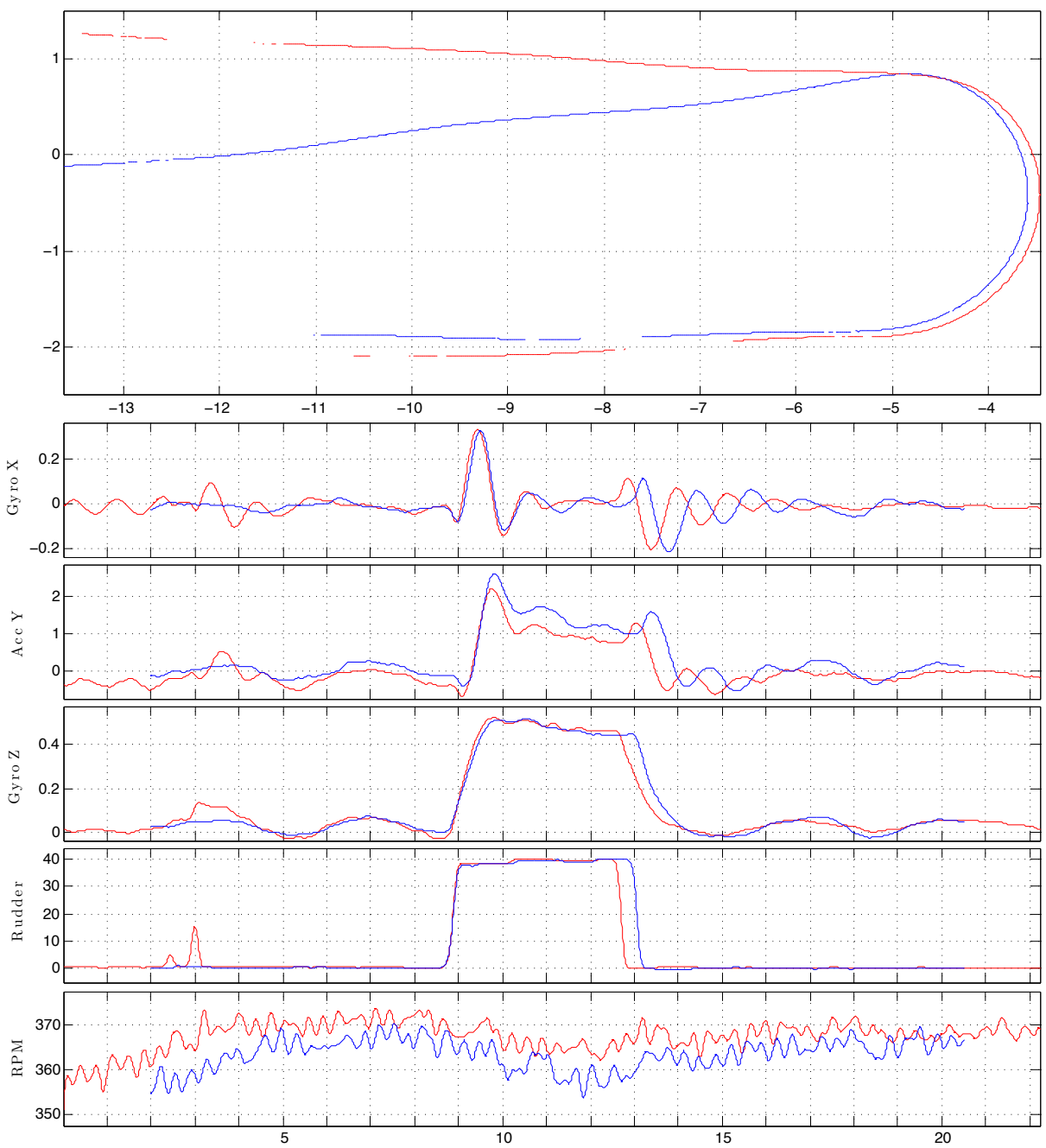

Figure 6.10: Two representative short datasets with U-turn maneuvers that are time synchronized to show the differences in behavior. The red lines correspond to the nominal dataset and the blue lines correspond to the dataset with a $m=0.200 \mathrm{~kg}$ additional load positioned $\bar{z}_{m}=-0.256 \mathrm{~m}$ from the bodyfixed coordinate system. Note the nonlinear behavior of the dynamics while turning or leaving the turn, for example, the difference in damping between times 10 to $13 \mathrm{~s}$ and times 15 to $18 \mathrm{~s}$.

\subsection{Analysis of Data}

Figure 6.10 shows two representative datasets that are time-synchronized to show the difference between the nominal and the additional load dataset. The additional load was $0.200 \mathrm{~kg}$ and located $-0.256 \mathrm{~m}$ from the body-fixed coordinate system. These datasets were collected without waves but there were still disturbances due to reflection of the bow-wave on the basin side walls. There are several interesting features that can be seen in the figure. 
- There is a visible difference in the acceleration measurement between the datasets due to the additional mass, for instance, between times 10 to $14 \mathrm{~s}$. This is more clear if the angular velocity around the roll axis is plotted against the acceleration measurement, see Figure 6.11. This difference is probably due to the larger stationary angle in the loaded case.

- The roll dynamics was nonlinear. For example, note that the damping was different between times 10 to $13 \mathrm{~s}$ and times 15 to $18 \mathrm{~s}$. The reason for this difference is unclear. One explanation might be that a larger part of the hull is in contact with the water during a turn.

- The non-minimum phase behavior is visible both going into the turn and going out of the turn.

- The propeller speed decreased during the turn which indicates that the speed should also have decreased. This is verified by the decrease in sideways acceleration from times 10 to $14 \mathrm{~s}$.

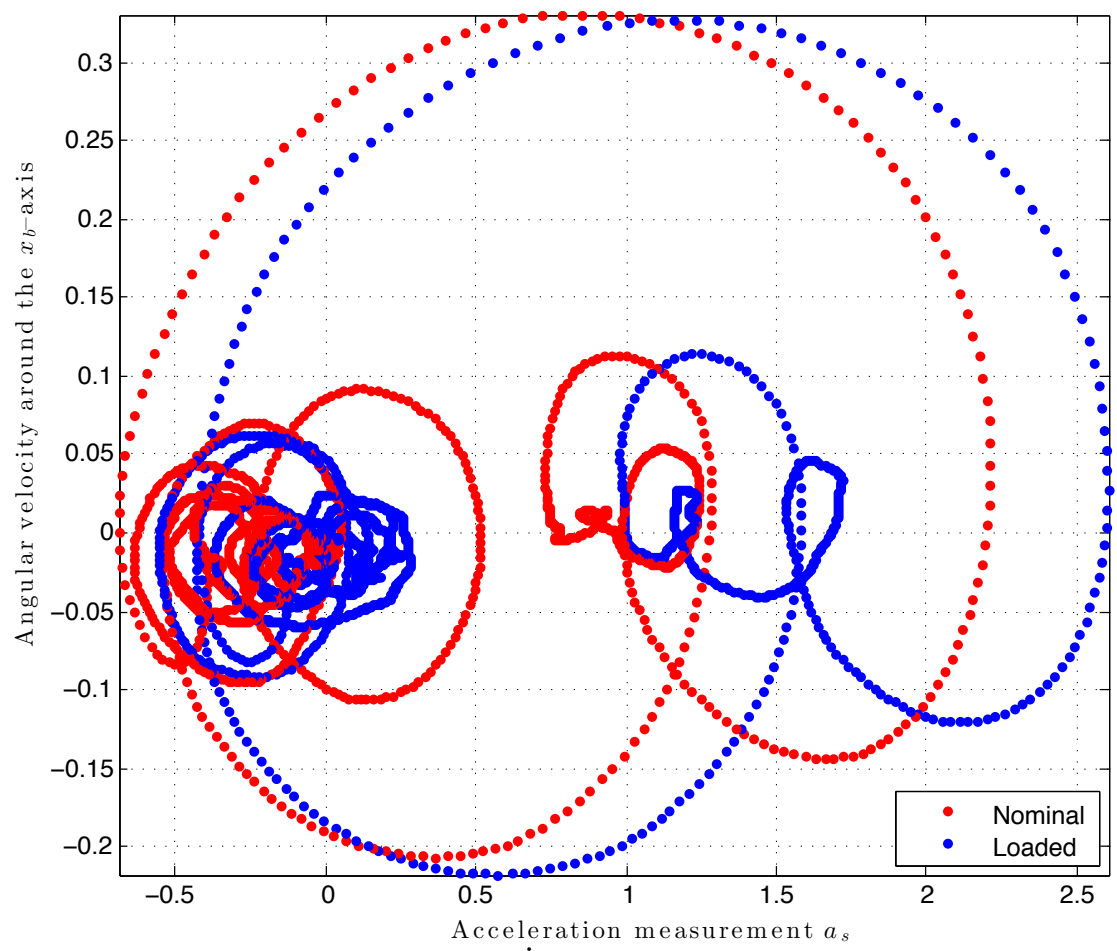

Figure 6.11: The angular velocity $\dot{\phi}$ around the $x_{b}$-axis plotted against the acceleration measurement $a_{s}$. Note that the angular velocities were roughly the same, but that the acceleration was increased during the turn when there was an additional load. Note also that the stationary point for the ship traveling straight is around $(0,0)$ and that the stationary point during a turn is somewhere around $(1,0)$. See also the acceleration measurement in Figure 6.10. 
Table 6.3: A summary of the datasets used to estimate the parameters of the input roll subsystem (5.20). The dataset number is only used as an identification number. All datasets were sampled at $100 \mathrm{~Hz}$, low-pass filtered and down-sampled to $50 \mathrm{~Hz}$ before estimation. The corresponding datasets can be seen in the figures indicated in the last column. Note that the number of samples is before downsampling. See Table 6.2 for an explanation of the loading cases.

\begin{tabular}{ccccc}
\hline Load Case & Number & Length & Samples & Shown In \\
\hline Nominal & 1 & $63.2 \mathrm{~s}$ & 6301 & Figure 6.12 \\
\hline \multirow{2}{*}{$0.200 \mathrm{~kg}$} & 2 & $36.1 \mathrm{~s}$ & 3601 & Figure 6.13 \\
& 3 & $51.1 \mathrm{~s}$ & 5101 & Figure 6.14 \\
\hline $0.400 \mathrm{~kg}$ & 4 & $65.5 \mathrm{~s}$ & 6540 & Figure 6.15 \\
\hline
\end{tabular}

\subsection{Estimation Results Under Moderate Sea Conditions}

This section describes the results from estimation of the parameters in the input roll subsystem (5.20) under moderate sea conditions. The experiments were executed with the wave maker turned off. However, there were clear process disturbances due to reflections of the bow waves on the sidewalls of the basin. The data were collected according to the description in Section 6.3.

The results presented in this section were produced using the datasets listed in Table 6.3. In contrast to the short datasets shown in Figure 6.10, the long datasets show less variations in the damping between the stationary mode going straight and the stationary mode in turn. One explanation might be a lower average speed, and hence a smaller difference in speed entering and leaving the turn, since there are multiple turns after each other. In the short datasets, the ship had time to build up its speed over the whole length of the basin. The datasets in Table 6.3 were chosen since they were similar to each other, for example, having the same maximum rudder angle and similar lengths, and since there were no strong nonlinear effects visible in the data. All four datasets were sampled at $100 \mathrm{~Hz}$, the datasets were filtered through an FIR equiripple low-pass filter of order 20 with a cut-off frequency of $0.5 \mathrm{~Hz}$ and were down-sampled to $50 \mathrm{~Hz}$ resulting in a data length roughly between 1800-3300 samples per dataset. The datasets can be seen in Figures 6.12 to 6.15 .

The datasets were combined into three different cases according to Table 6.4. In each case, the parameters

$$
\tilde{\vartheta}_{p, 1}=[\underbrace{M, \bar{z}_{g}}_{\bar{\vartheta}_{p, 1}}, m_{c}, \bar{z}_{c}]^{T},
$$

defined in (5.64) were assumed to be known. 
Table 6.4: A summary of the different estimation cases. Columns 2-4 show to the datasets used in the estimation. The last column shows where the results are summarized. The first and second row correspond to a table and figure showing the iterations.

\begin{tabular}{ccccc}
\hline Case & Nominal Dataset & Calibration Dataset & Loaded Dataset & Results In \\
\hline 1 & Nominal -1 & $0.200 \mathrm{~kg}-2$ & $0.200 \mathrm{~kg}-3$ & $\begin{array}{c}\text { Table } 6.5 \\
\text { Figure } 6.16\end{array}$ \\
\hline 2 & Nominal -1 & $0.200 \mathrm{~kg}-3$ & $0.400 \mathrm{~kg}-4$ & $\begin{array}{c}\text { Table } 6.8 \\
\text { Figure } 6.18\end{array}$ \\
\hline 3 & Nominal -1 & $0.200 \mathrm{~kg}-2$ & $0.400 \mathrm{~kg}-4$ & $\begin{array}{c}\text { Table } 6.9 \\
\text { Figure } 6.19\end{array}$ \\
\hline
\end{tabular}

Several different noise models were tried and the noise models used to produce the results in this section were

$$
\bar{H}_{d}^{i}\left(\mathrm{q}, \hat{\eta}^{i}\right)=\frac{1}{1+\bar{\alpha}_{1} \mathrm{q}^{-1}+\bar{\alpha}_{2} \mathbf{q}^{-2}}
$$

where $\left(1+\bar{\alpha}_{1} \mathrm{q}^{-1}+\bar{\alpha}_{2} \mathrm{q}^{-2}\right)$ is the denominator of $G_{d}\left(\mathrm{q}, \bar{\vartheta}_{p}^{i}\right)$, which corresponds to the prefilter

$$
L^{i}\left(\mathrm{q}, \bar{\vartheta}_{p}^{i}\right)=1
$$

In the initial step, the transfer functions in (5.85) were estimated using ARX model structures with $n_{a}=4$ and $n_{b}=5$. The vectors of instruments (5.87) in the initial steps were created using the constants $n_{y}=16, n_{a_{s}}=16, n_{r}=16$ and $n_{\delta}=2$ for each dataset.

The initial conditions were in all cases assumed to be

$$
\begin{aligned}
\tilde{\vartheta}_{p, 2} & =\left[k, A_{x}, d, K_{r}, K_{\dot{v}}, K_{\delta}, z_{f}, m, \bar{z}_{m}\right]^{T} \\
& =[9,0.5,0.1,-0.4,1,-0.4,-0.018,0,0]^{T}
\end{aligned}
$$

In the refining steps, the noise models were assumed to be given by (6.3) and the inputs and the output were simulated using (5.91). The vectors of instruments (5.95) were created using the constants $n_{y}=16, n_{a_{s}}=16, n_{r}=16$ and $n_{\delta}=2$ for each dataset, i.e. the same as in the initial step.

Algorithm 5.1 was then applied to each case in Table 6.4 and the results can be seen in Tables 6.5, 6.8 and 6.9, and Figures 6.16, 6.16 and 6.19. Note that the estimated parameters were roughly the same in all three cases. As expected, Case 1 gave the best results, probably since both the calibration and the loaded dataset had the same additional mass. It should however be noted that in Cases 2 and 3 the largest relative error, $16.6 \%$, corresponds to 66.5 gram error in the mass estimation. For more details on each case, see Sections 6.5.1 and 6.5.2. 

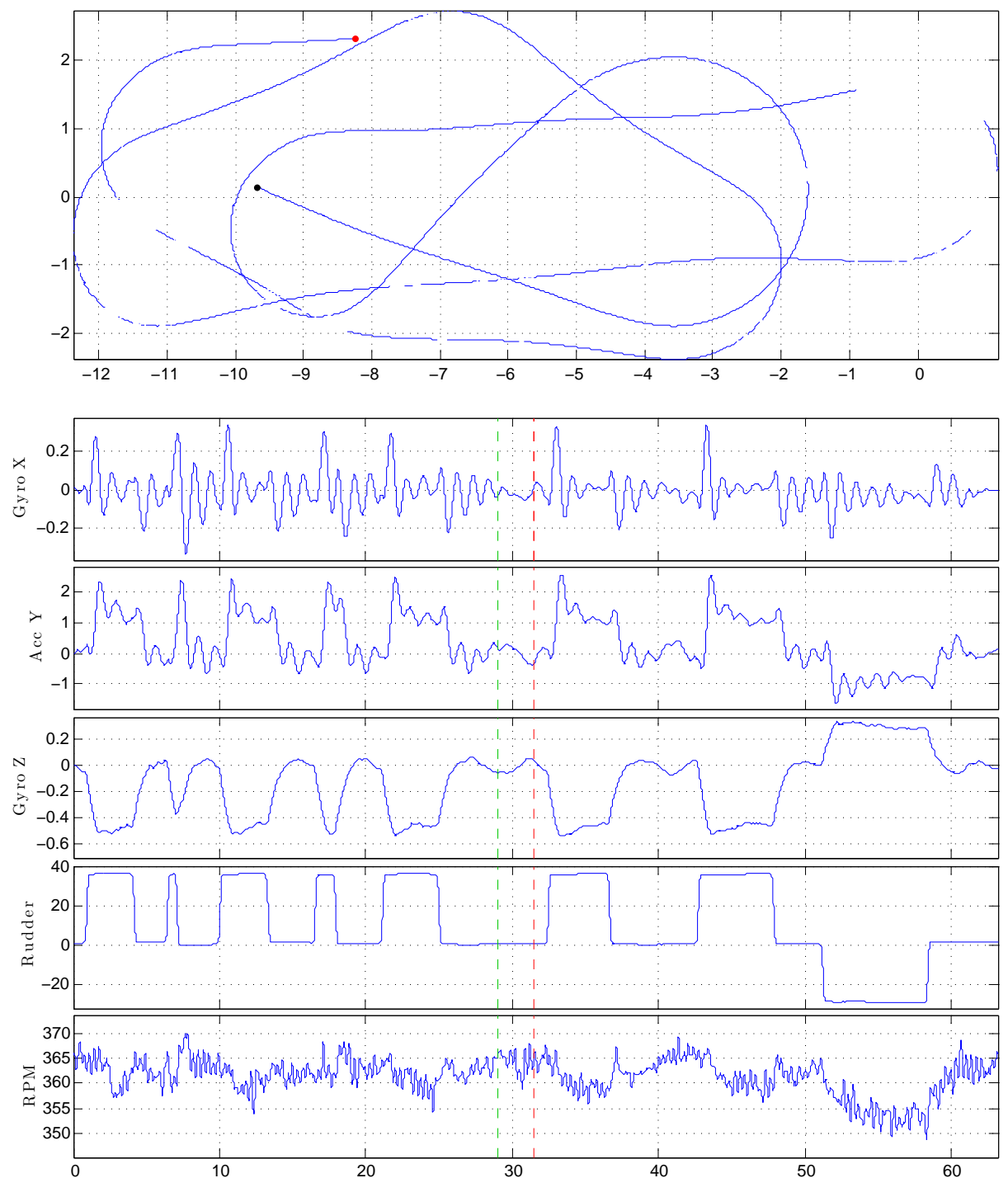

Figure 6.12: Nominal data - Dataset 1. The dashed red line corresponds to the end of Case 1(a) and the dashed green line corresponds to the start of Case 1(b). 

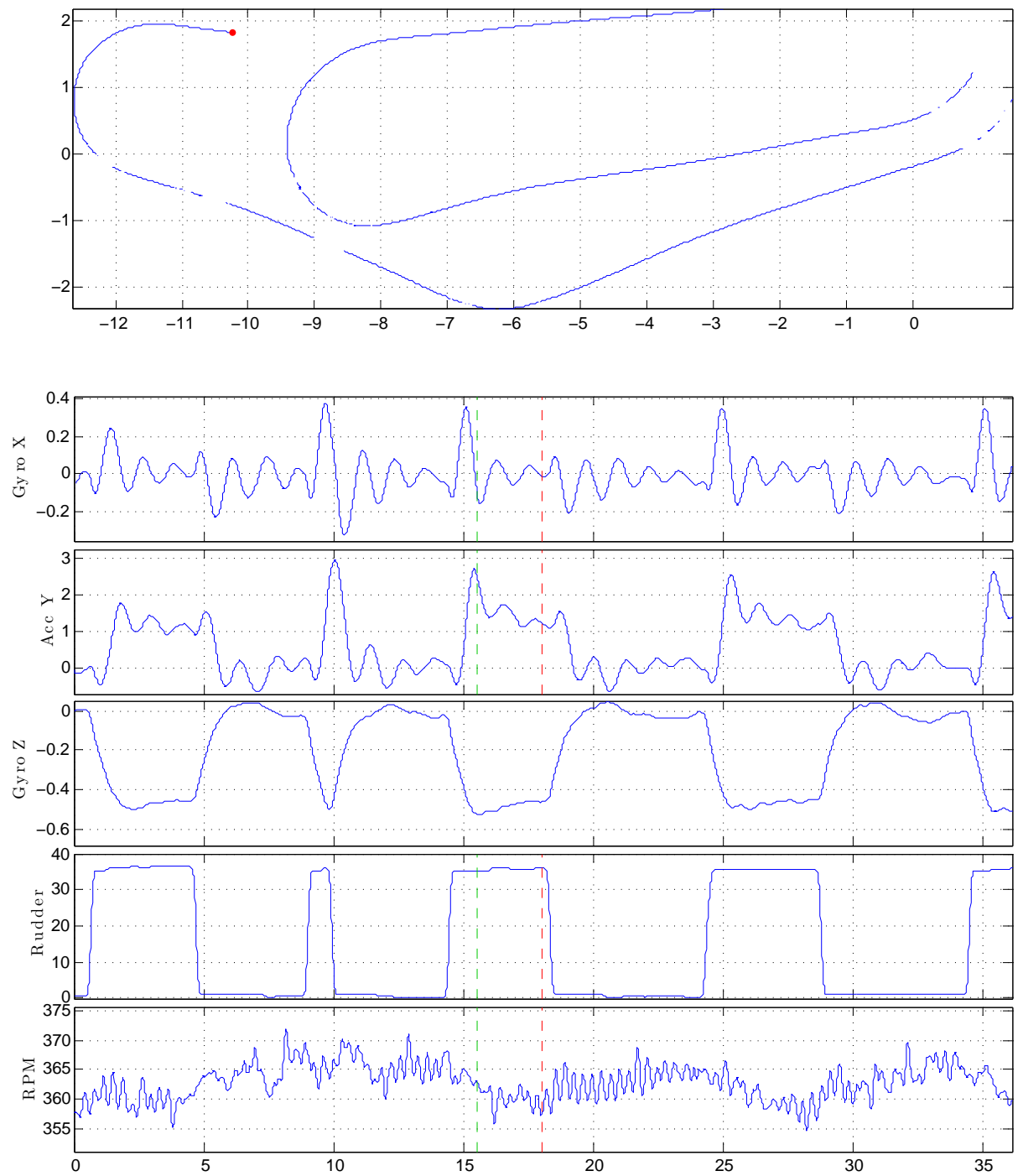

Figure 6.13: Loaded data $0.200 \mathrm{~kg}$ - Dataset 2. The dashed red line corresponds to the end of Case 1(a) and the dashed green line corresponds to the start of Case 1(b). 

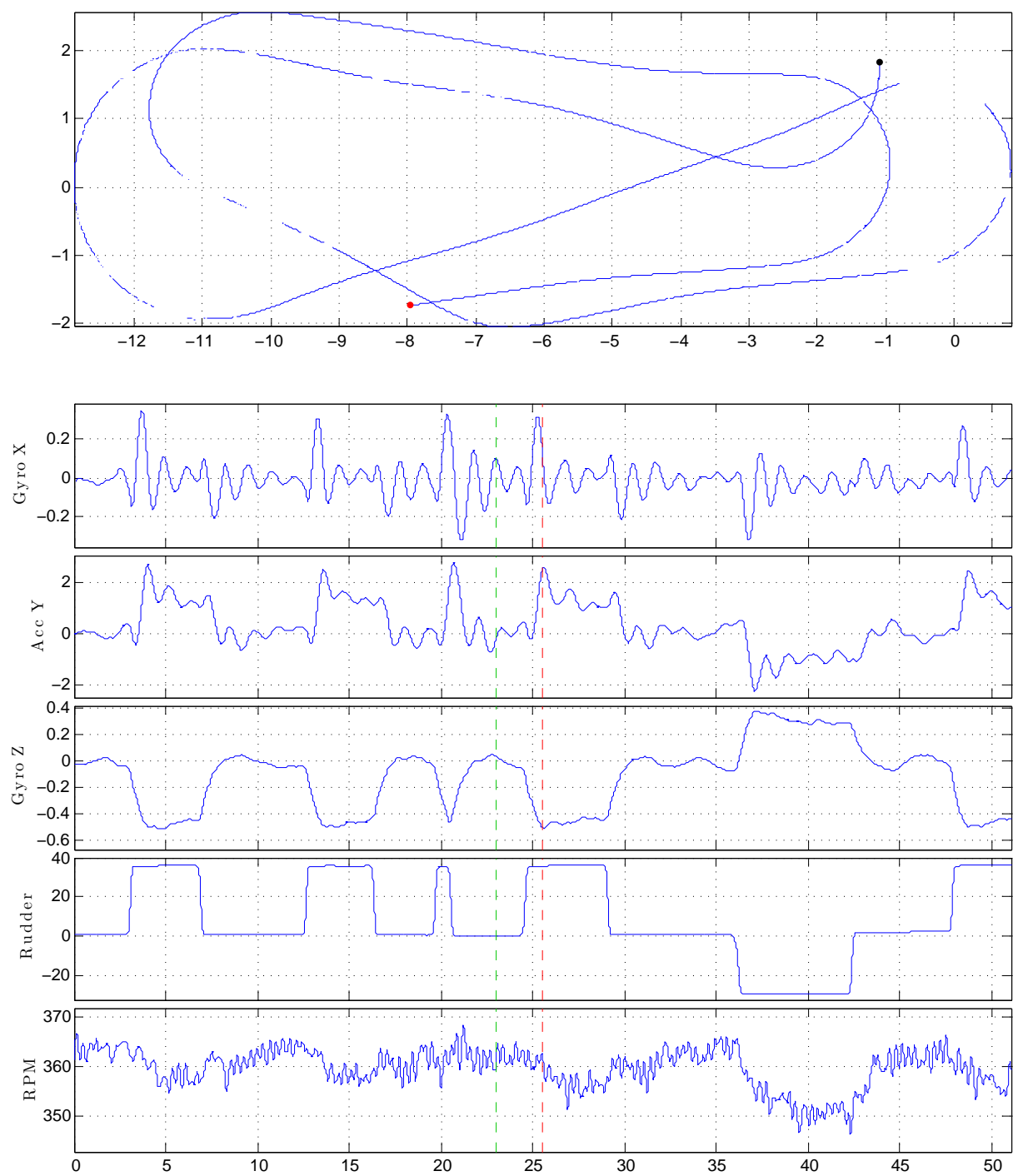

Figure 6.14: Loaded data $0.200 \mathrm{~kg}$ - Dataset 3. The dashed red line corresponds to the end of Case 1(a) and the dashed green line corresponds to the start of Case 1(b). 

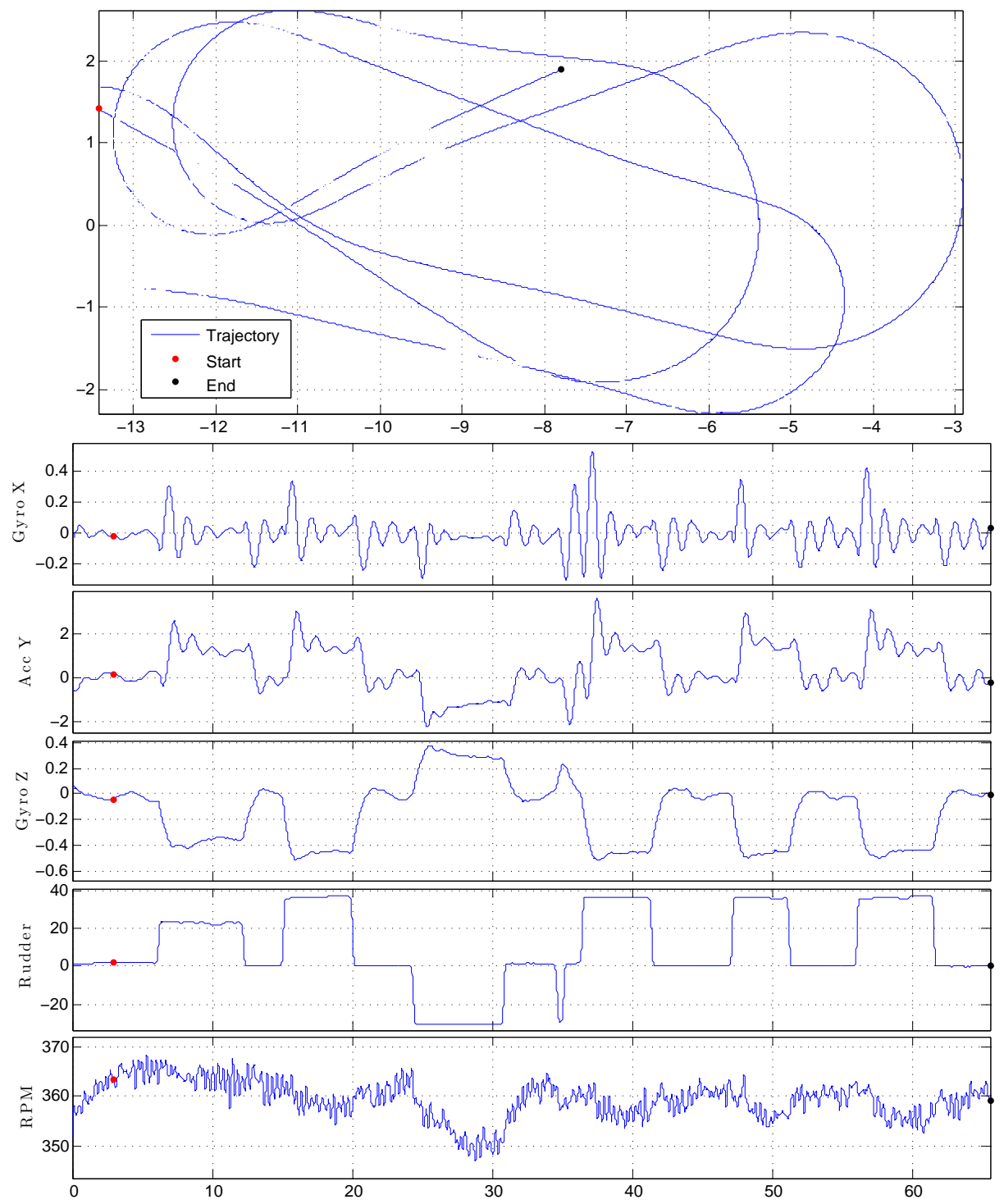

Figure 6.15: Loaded data $0.400 \mathrm{~kg}$ - Dataset 4 . 
Table 6.5: Estimation results with the datasets according to Case 1 in Table 6.4. The first column corresponds to: parameters $(P)$, true values $(T)$, initial values $(I)$, iteration (numbers) and the relative error $(E)$.

\begin{tabular}{|c|c|c|c|c|c|c|c|c|c|c|}
\hline $\mathrm{P}$ & $\bar{A}_{x}$ & $d$ & $k$ & $K_{\delta}$ & $K_{r}$ & $K_{\dot{v}}$ & $z_{f}$ & $m$ & $\bar{z}_{m}$ & \\
\hline $\mathrm{T}$ & - & - & - & - & - & - & - & 0.2000 & -0.2560 & \\
\hline I & 0.5000 & 0.1000 & 9.0000 & -0.4000 & -0.4000 & 1.0000 & -0.0180 & 0.0000 & 0.0000 & \\
\hline 0 & 0.1689 & 0.2982 & 9.0026 & -0.4994 & -2.1676 & 0.9385 & -0.0278 & 0.1388 & -0.3535 & \\
\hline 1 & 0.1578 & 0.2696 & 9.0056 & -0.4798 & -2.0079 & 0.9627 & -0.0294 & 0.1867 & -0.2739 & \\
\hline 2 & 0.1582 & 0.2683 & 9.0057 & -0.4821 & -2.0099 & 0.9604 & -0.0294 & 0.1986 & -0.2541 & \\
\hline 3 & 0.1583 & 0.2685 & 9.0057 & -0.4822 & -2.0101 & 0.9607 & -0.0294 & 0.1997 & -0.2530 & \\
\hline 4 & 0.1583 & 0.2685 & 9.0057 & -0.4822 & -2.0100 & 0.9607 & -0.0294 & 0.1997 & -0.2529 & \\
\hline 5 & 0.1583 & 0.2685 & 9.0057 & -0.4822 & -2.0100 & 0.9607 & -0.0294 & 0.1997 & -0.2529 & \\
\hline 6 & 0.1583 & 0.2685 & 9.0057 & -0.4822 & -2.0100 & 0.9607 & -0.0294 & 0.1997 & -0.2529 & \\
\hline 7 & 0.1583 & 0.2685 & 9.0057 & -0.4822 & -2.0100 & 0.9607 & -0.0294 & 0.1997 & -0.2529 & \\
\hline 8 & 0.1583 & 0.2685 & 9.0057 & -0.4822 & -2.0100 & 0.9607 & -0.0294 & 0.1997 & -0.2529 & \\
\hline $\mathrm{E}$ & - & - & - & - & - & - & - & 0.1338 & 1.2120 & $\%$ \\
\hline
\end{tabular}

\subsubsection{Case 1 - Equal Load in Calibration and Loaded Datasets}

The results for Case 1 can be seen in Table 6.5 and Figure 6.16. As expected, this case had the best results of all the cases in Table 6.4, probably since the calibration dataset was similar to the loaded dataset, i.e. having the same weight and position. The estimated mass was less than $1 \mathrm{~g}$ away from the true value and position had an error of less than $4 \mathrm{~mm}$.

For comparison, the constrained LS estimator, i.e. Approach 5.3 with $\Phi^{i}$ and $Y^{i}$ according to (4.48), was used to compute the estimate and the result can be seen as $\mathrm{L}$ in Table 6.7 and there is quite a significant difference compared to the IV estimator.

More flexible prefilters were tested but gave worse results. In Figure 6.17 the normalized residuals are plotted together with the normalized and scaled absolute value of the rudder angle. It can be seen that there is a clear correlation between the large spikes and the turn entries (and also the exits if it is a short turn as it was around 3500). This indicated that there was unmodeled dynamics and this might have made a variance calculation misleading due to the deterministic components in the residuals.

Table 6.6: The result of the $L S$ estimate with the datasets according to Case 1. The first column corresponds to: parameters $(P)$, true values $(T)$, initial values $(I)$, Solution $(L)$ and the relative error $(E)$.

\begin{tabular}{|c|c|c|c|c|c|c|c|c|c|}
\hline $\mathrm{P}$ & $\bar{A}_{x}$ & $d$ & $k$ & $K_{\delta}$ & $K_{r}$ & $K_{\dot{v}}$ & $z_{f}$ & $m$ & $\bar{z}_{m}$ \\
\hline $\mathrm{T}$ & - & - & - & - & - & - & - & 0.2000 & -0.2560 \\
\hline I & 0.5000 & 0.1000 & 9.0000 & -0.4000 & -0.4000 & 1.0000 & -0.0180 & 0.0000 & 0.0000 \\
\hline $\mathrm{L}$ & 0.1810 & 0.2739 & 9.0125 & -0.5828 & -2.1185 & 0.7861 & -0.0248 & 0.0574 & -0.6474 \\
\hline $\mathrm{E}$ & - & - & - & - & - & - & - & 71.2819 & $152.8834 \%$ \\
\hline
\end{tabular}



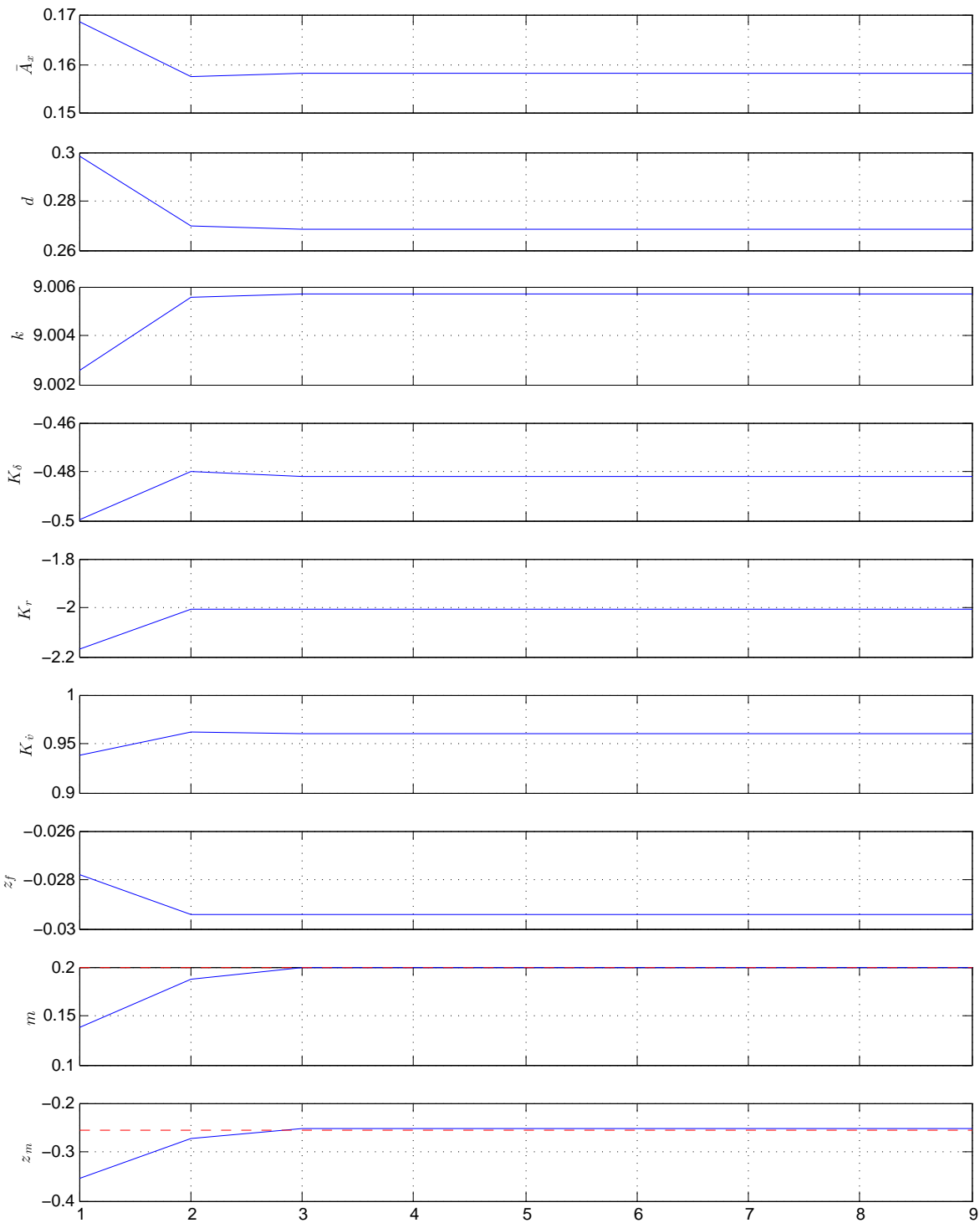

Figure 6.16: Estimation results with the datasets according to Case 1 in Table 6.4. The parameter values are plotted as functions of the iterations. Blue corresponds to estimated value and if a true value is known, it is shown as a dashed red line. The parameter values in each iteration are shown in Table 6.5. 


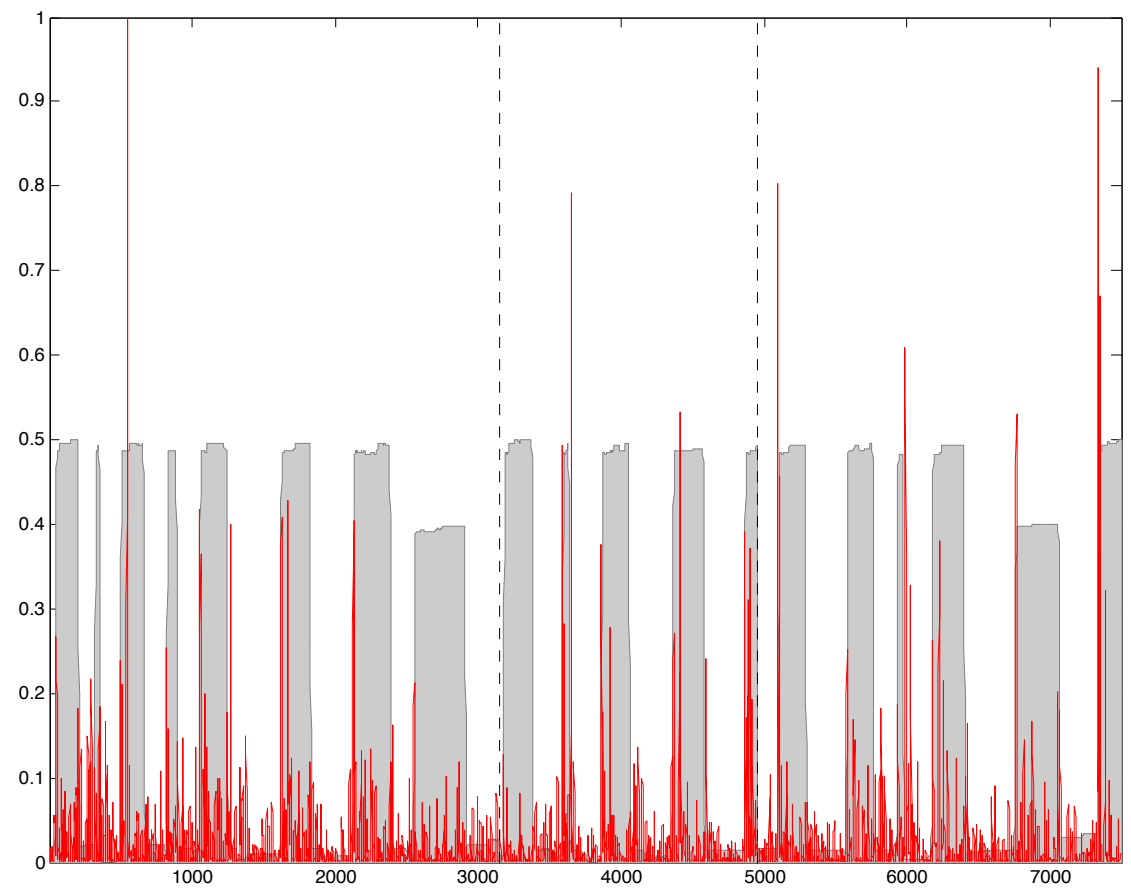

Figure 6.17: The residuals for the stacked three datasets of Case 1. The black dashed vertical lines show the separation between the datasets. Red: The normalized residuals. Gray: The absolute value of the rudder angle normalized and divided by two. Note that the area between the rudder angle and the $x$-axis are filled in light gray to make the plot easier to read. Note that there are spikes at almost all the turn entries, which indicates that the model was not capturing all the dynamics. The magnitudes of these spikes are around 10 times larger than the average residual.

To test the variation of the IV estimator the datasets of Case 1 were split into two cases. Case 1(a) roughly corresponded to the first half of the datasets and Case 2(b) roughly corresponded to the last half of the datasets. The end of the datasets of Case 1(a) are indicated as the dashed red line in Figures 6.12-6.14. In the same way, the start of the datasets of Case 1(b) are indicated as the dashed green line in Figures 6.12-6.14. In Case 1(a), the data lengths were 1576, 901 and 1276 samples after decimation for the nominal, calibration and loaded datasets, respectively. For Case 1(b), the corresponding numbers were 1701, 1026 and 1401. The parameters were estimated for both Cases 1(a) and 1(b), and the results can be seen with the indices A and B in Table 6.7. The maximum relative error, $54 \%$ was quite large, however, it corresponds to less than $1 \%$ of the total mass of the ship. Also, note that there might be a finite data effect since the datasets are quite short. 
Table 6.7: A: Estimation results with the datasets according to Case 1(a). B: Estimation results with the datasets according to Case 1 with the second half of the datasets. The first column corresponds to: parameters $(P)$, true values $(T)$, initial values $(I)$ and the relative error $(E)$.

\begin{tabular}{ccccccccccc}
\hline $\mathrm{P}$ & $\bar{A}_{x}$ & $d$ & $k$ & $K_{\delta}$ & $K_{r}$ & $K_{\dot{v}}$ & $z_{f}$ & $m$ & $\bar{z}_{m}$ & \\
\hline $\mathrm{T}$ & - & - & - & - & - & - & - & 0.2000 & -0.2560 & \\
$\mathrm{I}$ & 0.5000 & 0.1000 & 9.0000 & -0.4000 & -0.4000 & 1.0000 & -0.0180 & 0.0000 & 0.0000 & \\
\hline $\mathrm{A}$ & 0.1552 & 0.2411 & 8.9986 & -0.4821 & -2.0227 & 0.9376 & -0.0294 & 0.1629 & -0.2977 & \\
$\mathrm{E}$ & - & - & - & - & - & - & - & 18.5572 & 16.2706 & $\%$ \\
\hline $\mathrm{B}$ & 0.2234 & 0.4395 & 8.9967 & -0.5042 & -2.3138 & 1.0350 & -0.0246 & 0.3005 & -0.1951 & \\
$\mathrm{E}$ & - & - & - & - & - & - & - & 50.2588 & 23.7755 & $\%$ \\
\hline
\end{tabular}




\subsubsection{Cases 2 and 3 - Lighter Load in the Calibration Dataset}

Cases 2 and 3 were more realistic cases in a sense that the masses and the centers of gravity were different between the calibration and loaded datasets. The estimation results can be seen in Table 6.8 and Figure 6.18 for Case 2, and Table 6.9 and Figure 6.19 for Case 3. The largest relative error in the mass estimation was $16.6 \%$ which corresponds to $66.5 \mathrm{~g}$ or $0.3 \%$ of the total mass of the ship. Cases 2 and 3 had the same nominal and loaded datasets but different calibration datasets which was another indication that the variation was acceptable for the IV estimator.

For comparison the constrained LS estimator, i.e. Approach 5.3 with $\Phi^{i}$ and $Y^{i}$ according to (4.48), was used to compute the estimate and the result can be seen as $\mathrm{L}$ in Tables 6.10 and 6.11. In both cases, the IV estimator performed better.

Table 6.8: Estimation results with the datasets according to Case 2 in Table 6.4. The first column corresponds to: parameters $(P)$, true values $(T)$, initial values $(I)$, iteration (numbers) and the relative error $(E)$.

\begin{tabular}{|c|c|c|c|c|c|c|c|c|c|}
\hline $\mathrm{P}$ & $\bar{A}_{x}$ & $d$ & $k$ & $K_{\delta}$ & $K_{r}$ & $K_{\dot{v}}$ & $z_{f}$ & $m$ & $\bar{z}_{m}$ \\
\hline $\mathrm{T}$ & - & - & - & - & - & - & - & 0.4000 & -0.2680 \\
\hline I & 0.5000 & 0.1000 & 9.0000 & -0.4000 & -0.4000 & 1.0000 & -0.0180 & 0.0000 & 0.0000 \\
\hline 0 & 1685 & 0.2983 & 9.0015 & -0.4709 & -2.1358 & 0.9423 & -0.0280 & 0.3389 & -0.2742 \\
\hline 1 & 0.1554 & 0.2657 & 9.0070 & -0.4398 & -1.9793 & 0.9411 & -0.0294 & 0.3676 & -0.2426 \\
\hline 2 & 0.1566 & 0.2667 & 9.0067 & -0.4434 & -1.9911 & 0.9406 & -0.0293 & 0.3693 & -0.2424 \\
\hline 3 & 0.1566 & 0.2668 & 9.0067 & -0.4433 & -1.9910 & 0.9408 & -0.0293 & 0.3689 & -0.2426 \\
\hline 4 & 0.1566 & 0.2668 & 9.0067 & -0.4433 & -1.9911 & 0.9408 & -0.0293 & 0.3690 & -0.2426 \\
\hline 5 & 0.1566 & 0.2668 & 9.0067 & -0.4433 & -1.9911 & 0.9408 & -0.0293 & 0.3690 & -0.2 \\
\hline 6 & 0.1566 & 0.2668 & 9.0067 & -0.4433 & -1.9911 & 0.9408 & -0.0293 & 0.3690 & -0.2426 \\
\hline 7 & 0.1566 & 0.2668 & 9.0067 & -0.4433 & -1.9911 & 0.9408 & -0.0293 & 0.3690 & -0.2426 \\
\hline 8 & 0.1566 & 0.2668 & 9.0067 & -0.4433 & -1.9911 & 0.9408 & -0.0293 & 0.3690 & -0.2426 \\
\hline 9 & 0.1566 & 0.2668 & 9.0067 & -0.4433 & -1.9911 & 0.9408 & -0.0293 & 0.3690 & -0.2426 \\
\hline 10 & 0.1566 & 0.2668 & 9.0067 & -0.4433 & -1.9911 & 0.9408 & -0.0293 & 0.3690 & -0.2426 \\
\hline 11 & 0.1566 & 0.2668 & 9.0067 & -0.4433 & -1.9911 & 0.9408 & -0.0293 & 0.3690 & -0.2426 \\
\hline 12 & 0.1566 & 0.2668 & 9.0067 & -0.4433 & -1.9911 & 0.9408 & -0.0293 & 0.3690 & -0.2426 \\
\hline $\mathrm{E}$ & - & - & - & - & - & - & - & 7.7532 & 9.4842 \\
\hline
\end{tabular}


Table 6.9: Estimation results with the datasets according to Case 3 in Table 6.4. The first column corresponds to: parameters $(P)$, true values $(T)$, initial values $(I)$, iteration (numbers) and the relative error $(E)$.

\begin{tabular}{|c|c|c|c|c|c|c|c|c|c|c|}
\hline $\mathrm{P}$ & $\bar{A}_{x}$ & $d$ & $k$ & $K_{\delta}$ & $K_{r}$ & $K_{\dot{v}}$ & $z_{f}$ & $m$ & $\bar{z}_{m}$ & \\
\hline $\mathrm{T}$ & - & - & - & - & - & - & - & 0.4000 & -0.2680 & \\
\hline I & 0.5000 & 0.1000 & 9.0000 & -0.4000 & -0.4000 & 1.0000 & -0.0180 & 0.0000 & 0.0000 & \\
\hline 0 & 0.1693 & 0.2973 & 8.9967 & -0.4605 & -2.1405 & 0.9585 & -0.0282 & 0.2814 & -0.3220 & \\
\hline 1 & 0.1569 & 0.2674 & 9.0021 & -0.4351 & -1.9960 & 0.9527 & -0.0294 & 0.3299 & -0.2698 & \\
\hline 2 & 0.1571 & 0.2674 & 9.0021 & -0.4382 & -1.9987 & 0.9518 & -0.0294 & 0.3326 & -0.2676 & \\
\hline 3 & 0.1571 & 0.2675 & 9.0021 & -0.4383 & -1.9995 & 0.9516 & -0.0294 & 0.3335 & -0.2671 & \\
\hline 4 & 0.1571 & 0.2675 & 9.0021 & -0.4383 & -1.9995 & 0.9516 & -0.0294 & 0.3335 & -0.2671 & \\
\hline 5 & 0.1571 & 0.2675 & 9.0021 & -0.4383 & -1.9995 & 0.9516 & -0.0294 & 0.3335 & -0.2671 & \\
\hline 6 & 0.1571 & 0.2675 & 9.0021 & -0.4383 & -1.9995 & 0.9516 & -0.0294 & 0.3335 & -0.2671 & \\
\hline $\mathrm{E}$ & - & - & - & - & - & - & - & 16.6312 & 0.3286 & 70 \\
\hline
\end{tabular}

Table 6.10: The result of the LS estimate with the datasets according to Case 2 in Table 6.4. For comparison with IV estimator, see Table 6.8. The first column corresponds to: parameters $(P)$, true values $(T)$, initial values $(I), L S$ solution $(L)$ and the relative error $(E)$.

\begin{tabular}{|c|c|c|c|c|c|c|c|c|c|}
\hline $\mathrm{P}$ & $\bar{A}_{x}$ & $d$ & $k$ & $K_{\delta}$ & $K_{r}$ & $K_{\dot{v}}$ & $z_{f}$ & $m$ & $\bar{z}_{m}$ \\
\hline $\mathrm{T}$ & - & - & - & - & - & - & - & 0.4000 & -0.2680 \\
\hline I & 0.5000 & 0.1000 & 9.0000 & -0.4000 & -0.4000 & 1.0000 & -0.0180 & 0.0000 & 0.0000 \\
\hline $\mathrm{L}$ & 0.1882 & 0.2830 & 9.0108 & -0.5827 & -2.1800 & 0.8256 & -0.0247 & 0.8602 & -0.1221 \\
\hline $\mathrm{E}$ & - & - & - & - & - & - & - & 115.0549 & $54.4553 \%$ \\
\hline
\end{tabular}

Table 6.11: The result of the $L S$ estimate with the datasets according to Case 3 in Table 6.4. For comparison with IV estimator, see Table 6.9. The first column corresponds to: parameters $(P)$, true values $(T)$, initial values $(I), L S$ solution $(L)$ and the relative error $(E)$.

\begin{tabular}{|c|c|c|c|c|c|c|c|c|c|}
\hline $\mathrm{P}$ & $\bar{A}_{x}$ & $d$ & $k$ & $K_{\delta}$ & $K_{r}$ & $K_{\dot{v}}$ & $z_{f}$ & $m$ & $\bar{z}_{m}$ \\
\hline $\mathrm{T}$ & - & - & - & - & - & - & - & 0.4000 & -0.2680 \\
\hline I & 0.5000 & 0.1000 & 9.0000 & -0.4000 & -0.4000 & 1.0000 & -0.0180 & 0.0000 & 0.0000 \\
\hline $\mathrm{L}$ & 0.1817 & 0.2802 & 9.0099 & -0.5886 & -2.1624 & 0.8185 & -0.0251 & 0.5580 & -0.1826 \\
\hline $\mathrm{E}$ & - & - & - & - & - & - & - & 39.5013 & $31.8663 \%$ \\
\hline
\end{tabular}



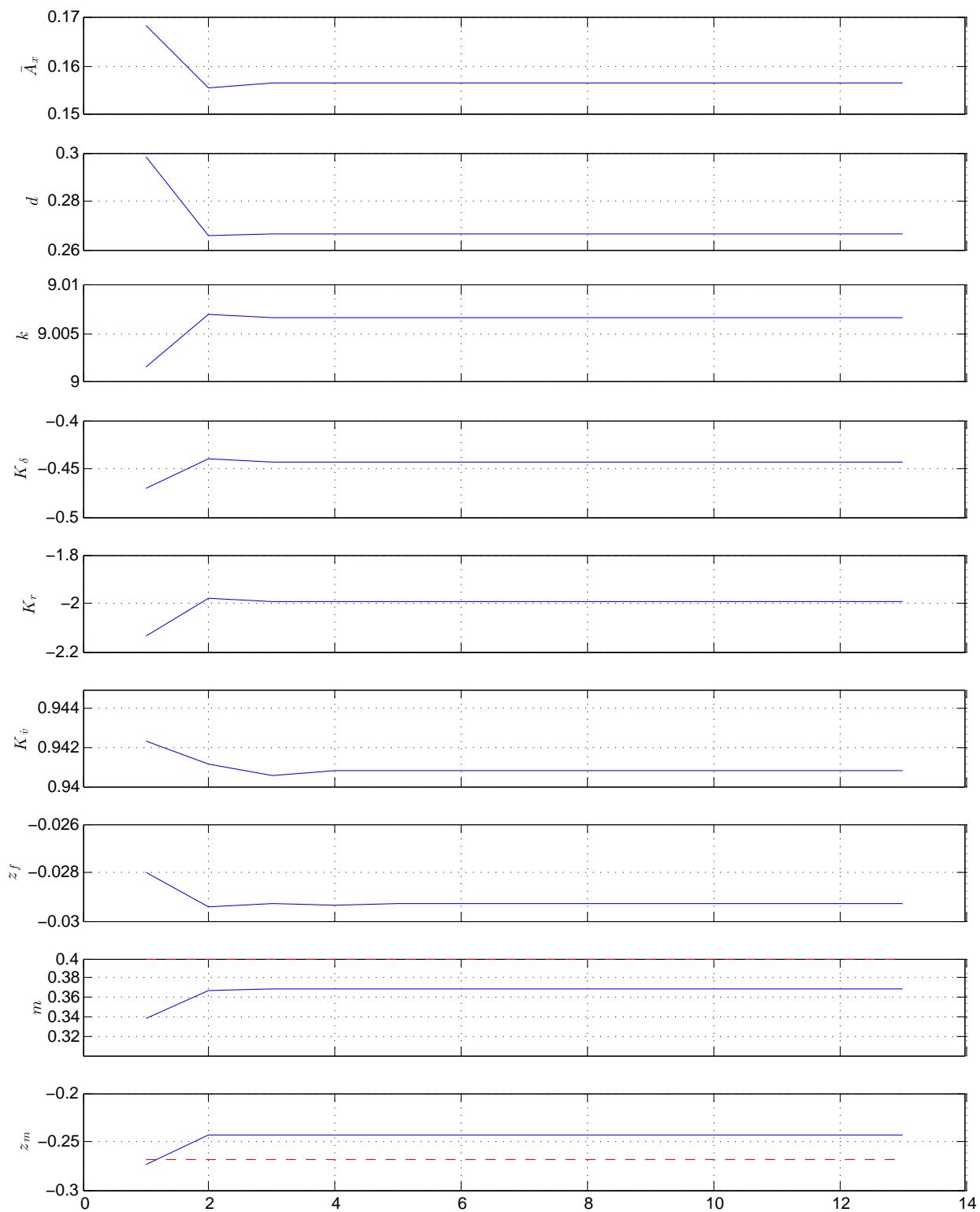

Figure 6.18: Estimation results with the datasets according to Case 2 in Table 6.4. The parameter values are plotted as functions of the iterations. Blue corresponds to estimated value and if a true value is known, it is shown as a dashed red line. The parameter values in each iteration are shown in Table 6.8. 

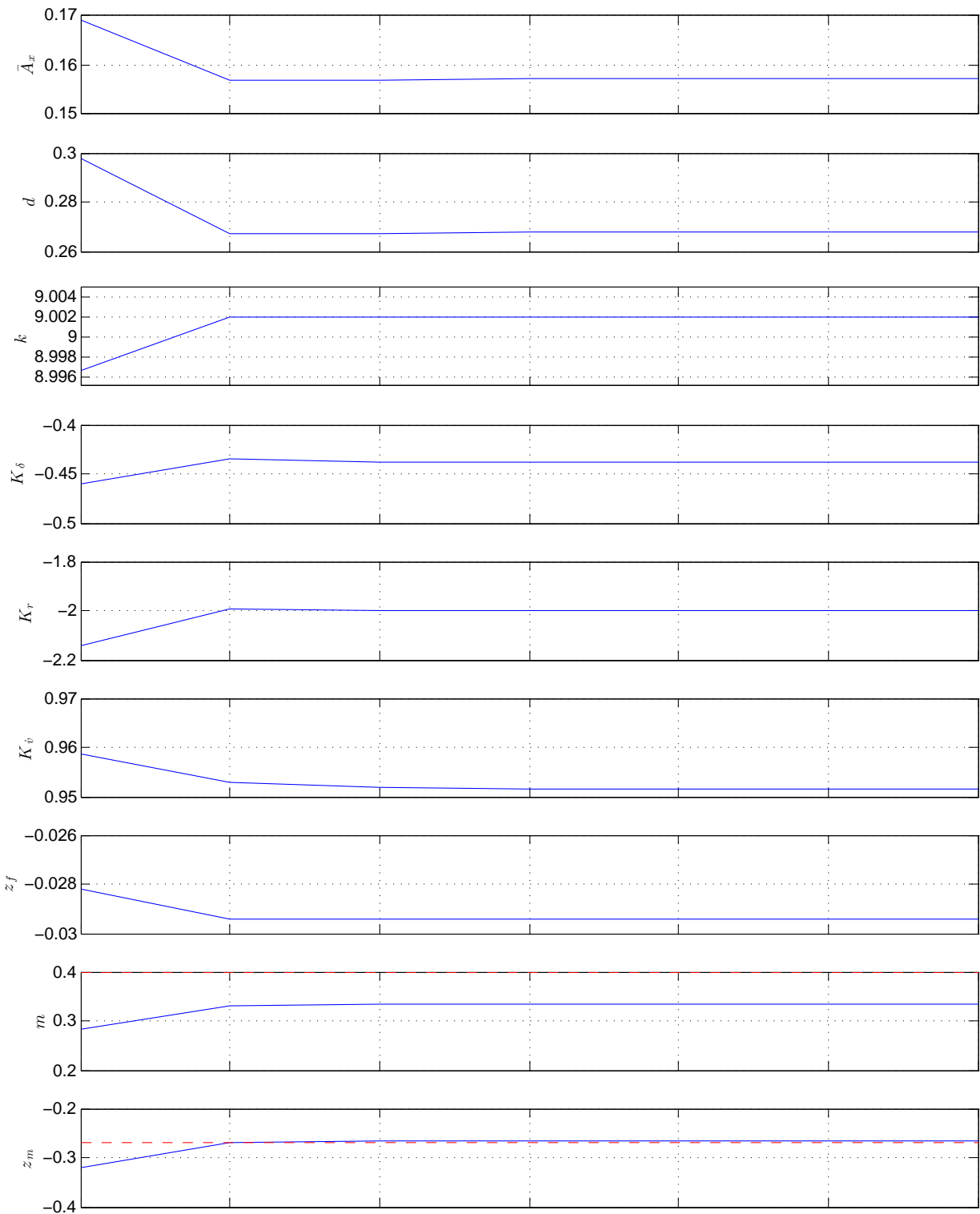

Figure 6.19: Estimation results with the datasets according to Case 3 in Table 6.4. The parameter values are plotted as functions of the iterations. Blue corresponds to estimated value and if a true value is known, it is shown as a dashed red line. The parameter values in each iteration are shown in Table 6.9. 



\section{Conclusions}

In this thesis we have used graybox models with indirect input measurements to estimate physical parameters. In particular, a ship's roll dynamics was studied and it was assumed that only motion measurements from an inertial measurement unit (IMU) together with measurements of the rudder angle were available. To understand the basic properties of the proposed method, it was applied to the approximate model (4.10) that despite its simplicity, shared the same properties as the roll dynamics of many vehicles, e.g. cars (Reineh et al., 2013), trucks, trains and ships. It was shown that a more complete ship model could be used as a starting point to derive a generalization of the approximate model (4.10), with the yaw-rate $r$ and rudder angle $\delta$ as additional inputs and a few extra ship-related parameters.

Identifiabiltiy analyses were performed on both the approximate model (4.10) in Section 4.2.1, and the generalized model (5.10) in Section 5.3. In both cases it was shown that only a subset of the unknown parameters could be estimated simultaneously. A physical interpretation is that this restriction is partly due to the force-mass ambiguity described in Section 4.1. For the generalized model (5.10) it was shown that an extra calibration dataset had to be introduced and that the use of multiple datasets could be used to overcome some of these identifiability restrictions.

The graybox formulation together with multiple datasets was used both in Section 4.4 and Section 5.5 to circumvent the restrictions that were shown in the identifiability analyses. In Section 4.4 the multi-stage method was introduced. It used the datasets in a sequential manner to introduce more information in each step of the method. Because of the extra complexity of the generalized model (5.10), a joint identification approach, that used several datasets simultaneously, was introduced in Section 5.5. The graybox formulation enabled fusion of data even though 
the data was collected for different system configurations. This approach is similar to merging segments of data from the same system configurations and is certainly applicable also in other types of problems than the ones described in this thesis.

The impact of disturbances were analyzed for the approximate model (4.10) in Section 4.3.2, and for the generalized model (5.10) in Section 5.2.2. The analyses showed that the estimation problem was similar to a closed-loop errors-invariable problem due to the use of the indirect input measurements.

An iterative closed-loop instrumental variable approach was formulated both for estimating the parameters in each step of the multi-stage method of Section 4.4 and for solving the joint identification problem in Section 5.5. The choice of the instrumental variable method was partly due to the subsystems viewpoint and partly due to the results of the disturbance analyses. The choices of instruments and prefilter are important for the performance and even though some promising results have been obtained, there is more to be understood. Future work should focus on understanding why the more flexible noise model did not improve the results seen in Section 6.5.

The experimental verification described in Section 6 gave promising results. However, there are still many possibilities for improvement and robustification.

To deal with the unmodeled dynamics hinted in Figure 6.17 extensions to the model should be considered. The complexity of the model should however still be kept low. It is not obvious which route to select. One extension to deal with the variation in damping shown in Figures $6.12-6.15$ is by the use of some kind of linear parameter varying (LPV) model or even a nonlinear function if that is not sufficient. Another extension could be a simple model of the surge speed dependency. 


\section{A}

\section{Calculating the Mass and Center of Mass Through Ballasting}

The identifiability analysis in Section 4.2.1 showed that it is only possible to estimate three-combinations of parameters given that the acceleration $a_{y}$ of the center of rotation is persistently exciting. As a first step, a static estimation procedure can be used to estimate the mass $M$, the center of mass $z_{g}$ and the spring coefficient $k$. These can be used together with IMU data from the nominal case to detect and estimate mass changes without the need to redo the static procedure.

In the harbor, or any other place where the dynamic pressure is not influencing the pressure sensors, the pressure sensors can be used to get an estimate of the draft and angle of the ship in relation to the waterline. These measurements can be used in combination with the geometric shape of the hull and the ballast system

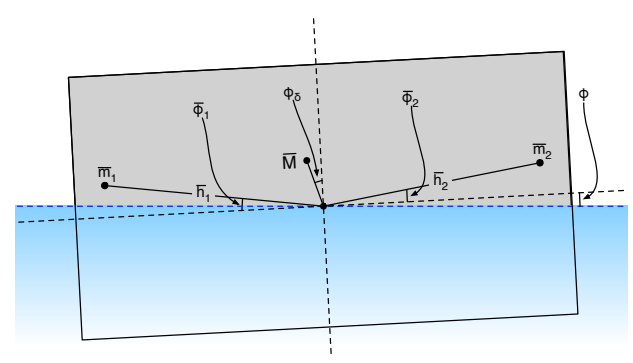

(a) The ship before ballasting

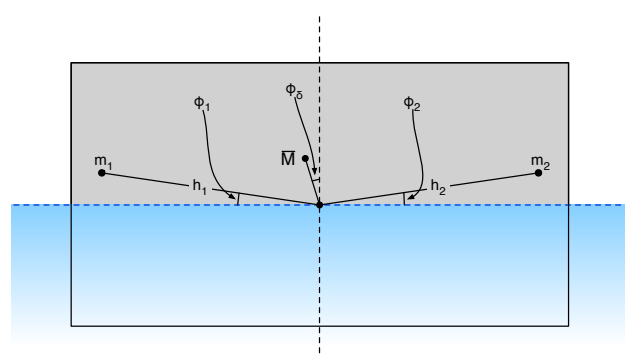

(b) The ship after ballasting

Figure A.1: The mass and position of a ship in mechanical equilibrium before and after ballasting. Note that the second case only have to be another stationary angle $\phi$ and does not have to be zero angle. 
to get an estimate of the mass and the CM. Assuming that the ballast system's tanks have a known weight and position, the mass and the CM can be found using the fact that the system is in mechanical equilibrium. Moment equilibrium around the CF of Figure A.1(a) gives

$$
\bar{M} g \bar{H} \sin \left(\phi+\phi_{\delta}\right)-\bar{m}_{2} g \bar{h}_{2} \cos \left(\phi+\bar{\phi}_{2}\right)+\bar{m}_{1} g \bar{h}_{1} \cos \left(\bar{\phi}_{1}-\phi\right)-\rho g \nabla \overline{G M}_{T} \sin (\phi)=0
$$

and secondly, force equilibrium in the world fixed $z$-axis gives

$$
\left(\bar{M}+\bar{m}_{1}+\bar{m}_{2}\right) g-\rho g \nabla=0
$$

where the distance to the unknown CM is calculated using the $y$ and $z$ position, i.e. $\bar{H}=\sqrt{\bar{y}_{g}^{2}+\bar{z}_{g}^{2}}$. However, there are three unknowns $\left(y_{g}, z_{g}\right.$ and $\left.\bar{M}\right)$ and two equations. Shifting the weight using the ballast system gives a new moment equilibrium and the needed third equation

$$
\bar{M} g \bar{H} \sin \left(\phi_{\delta}\right)-m_{2} g h_{2} \cos \left(\phi_{2}\right)+m_{1} g h_{1} \cos \left(\phi_{1}\right)=0
$$

As an example, assume a box shaped ship and a small change in the angle due to ballasting. This gives

$$
\overline{G M}_{T}=\frac{I_{T}}{\nabla}-\overline{B G}=\frac{I_{T}}{\nabla}-\sqrt{\left(H_{b, y}-y_{g}\right)^{2}+\left(H_{b, z}-z_{g}\right)^{2}}
$$

where $I_{T}=\frac{1}{12} B^{3} L, \nabla=L B D$ and $\overline{B G}$ is the distance between center of buoyancy (known) and center of gravity (unknown) (Fossen, 2011; Perez, 2005). The CM can now be calculated by

$$
y_{g}=\frac{S_{x z}}{M}=\frac{-\bar{M} \bar{H} \sin \left(\phi_{\delta}\right)-m_{1} h_{1} \cos \left(\phi_{1}\right)+m_{2} h_{2} \cos \left(\phi_{2}\right)}{\bar{M}+m_{1}+m_{2}}
$$

and

$$
z_{g}=\frac{S_{x y}}{M}=\frac{\bar{M} \bar{H} \cos \left(\phi_{\delta}\right)+m_{1} h_{1} \sin \left(\phi_{1}\right)+m_{2} h_{2} \sin \left(\phi_{2}\right)}{\bar{M}+m_{1}+m_{2}}
$$

where $S_{x y}$ and $S_{x z}$ are the first moments of area. Equation (A.1) - (A.6) can be solved for $\bar{M}, \bar{x}_{g}$ and $\bar{z}_{g}$, for instance, by using some numerical method. The total mass is given by

$$
M=\bar{M}+m_{1}+m_{2},
$$

the distance to the $\mathrm{CM}$ is

$$
H=\sqrt{y_{g}^{2}+z_{g}^{2}}
$$

and the spring coefficient is given by

$$
k=\rho g \nabla \overline{G M}_{T}-M g H
$$




\section{Bibliography}

J. Albersmeyer and M. Diehl. The lifted Newton method and its application in optimization. SIAM Journal on Optimization, 20(3):1655-1684, 2010. Cited on page 82.

M. Anguelova. Observability and identifiability of nonlinear systems with applications in biology. PhD thesis, Chalmers University of Technology, Gothenburg, Sweden, 2007. Cited on page 17.

L. Balcer. Location of ship rolling axis. Polish Maritime Research, pages 3-7, 2004. Cited on page 21 .

A. S. Bazanella, M. Gevers, and L. Miškovic. Closed-loop identification of MIMO systems: A new look at identifiability and experiment design. European Journal of Control, 16(3):228 - 239, 2010. ISSN 0947-3580. doi: 10.3166/ejc.16.228-239. Cited on pages 47 and 76 .

R. Bellman and K. Åström. On structural identifiability. Mathematical Biosciences, 7(3-4):329 - 339, 1970. ISSN 0025-5564. doi: 10.1016/0025-5564(70)90132-X. Cited on page 15 .

M. Blanke and A. C. Christensen. Rudder-roll damping autopilot robustness to sway-yaw-roll couplings. Rudder-Roll Damping Autopilot Robustness to SwayYaw-Roll Couplings, 1993. Cited on pages 26 and 30.

S. Diop and M. Fliess. Nonlinear observability, identifiability, and persistent trajectories. In Proceedings of the 30th IEEE Conference on Decision and Control, Brighton, England, 1991. doi: 10.1109/CDC.1991.261405. Cited on page 16.

I. Drummen. Experimental and Numerical Investigation of Nonlinear WaveInduced Load Effects in Containerships considering Hydroelasticity. PhD thesis, Norwegian University of Science and Technology, Trondheim, Norway, 2008. Cited on page 20 .

O. M. Faltinsen. Sea loads on ships and offshore structures. Cambridge University Press, Cambridge, 1990. ISBN 0521458706. Cited on pages 19 and 24.

O. M. Faltinsen. Hydrodynamics of High-Speed Marine Vehicles. Cambridge 
University Press, 2005. ISBN 9780521845687. Cited on pages 3, 24, 27, and 29.

U. Forssell. Closed-loop identification: Methods, theory, and applications. PhD thesis, Linköping University, Linköping, Sweden, 1999. Cited on pages 13 and 53.

U. Forssell and C. Chou. Efficiency of prediction error and instrumental variable methods for closed-loop identification. In Proceedings of the 37th IEEE Conference on Decision and Control, pages 1287-1288 vol.2, Tampa, Florida, USA, 1998. Cited on page 14.

T. I. Fossen. Nonlinear modelling and control of underwater vehicles. PhD thesis, Norwegian University of Science and Technology, Trondheim, Norway, 1991. Cited on pages 19 and 23.

T. I. Fossen. Handbook of Marine Craft Hydrodynamics and Motion Control. Wiley, 2011. ISBN 9781119991496. Cited on pages 3, 19, 20, 22, 23, 26, 28, 29, and 116.

T. Fujiwara and T. Haraguchi. Roll motion of Ro-Ro passenger ship with flooded vehicle deck. International Journal of Offshore and Polar Engineering, 15(2): 109-116, 2005. Cited on page 2.

M. Gevers, A. Bazanella, X. Bombois, and L. Miskovic. Identification and the information matrix: How to get just sufficiently rich? IEEE Transactions on Automatic Control, 54(12):2828-2840, Dec 2009. ISSN 0018-9286. doi: 10.1109/ TAC.2009.2034199. Cited on page 76 .

M. Gilson and P. Van den Hof. Instrumental variable methods for closed-loop system identification. Automatica, 41(2):241-249, 2005. Cited on page 14 .

M. Gilson, H. Garnier, P. J. Young, P. Van den Hof, et al. A refined IV method for closed-loop system identification. In Proceedings of the 14th IFAC Symposium on System Identification, pages 903-908, Newcastle, Australia, 2006. Cited on page 84 .

M. Gilson, H. Garnier, P. C. Young, and P. M. Van den Hof. Optimal instrumental variable method for closed-loop identification. IET Control Theory \& Applications, 5(10):1147-1154, 2011. Cited on page 14.

G. Golub and C. Van Loan. Matrix Computations. Johns Hopkins Studies in the Mathematical Sciences. Johns Hopkins University Press, 1996. ISBN 9780801854149. Cited on page 52.

R. Hermann and A. Krener. Nonlinear controllability and observability. IEEE Transactions on Automatic Control, 22(5):728-740, 1977. Cited on page 16.

T. Iseki and D. Terada. Bayesian estimation of directional wave spectra for ship guidance system. In Proceedings of the International Offshore and Polar Engineering Conference, volume 4, pages 577-582, 2001. Cited on page 3.

J. M. J. Journée and W. W. Massie. Offshore Hydromechanics. Delft University of Technology, 2001. Cited on pages 19 and 25. 
C. Källström, K. Åström, N. Thorell, J. Eriksson, and L. Sten. Adaptive autopilots for tankers. Automatica, 15(3):241 - 254, 1979. ISSN 0005-1098. doi: 10.1016/ 0005-1098(79)90042-6. Cited on page 8.

B. Lautrup. Physics of Continuous Matter: Exotic and Everyday Phenomena in the Macroscopic World. Taylor \& Francis, 2004. ISBN 9780750307529. Cited on pages 24 and 25 .

J. Linder and S. Lindkvist. Interactive multiobjective optimization with application to hot rolling mills. Master's thesis, Chalmers tekniska högskola, Gothenburg, Sweden, 2011. Cited on page 4.

J. Linder, M. Enqvist, and F. Gustafsson. A closed-loop instrumental variable approach to mass and center of mass estimation using IMU data. In Proceedings of the 53rd IEEE Conference on Decision \& Control, Los Angeles, CA, USA, December 2014a. Cited on page 54.

J. Linder, M. Enqvist, F. Gustafsson, and J. Sjöberg. Identifiability of physical parameters in systems with limited sensors. In Proceedings of the 19th IFAC World Congress, Cape Town, South Africa, August 2014b. Cited on page 54.

L. Ljung. System Identification: Theory for the User. Prentice Hall, 1999. ISBN 0136566952. Cited on pages 7, 10, 12, 13, 44, 47, 49, and 53 .

L. Ljung and T. Glad. On global identifiability for arbitrary model parametrizations. Automatica, 30(2):265 - 276, 1994. ISSN 0005-1098. doi: 10.1016/ 0005-1098(94)90029-9. Cited on pages 15 and 40.

J. Newman. Marine Hydrodynamics. Wei Cheng Cultural Enterprise Company, 1977. ISBN 9780262140263. Cited on page 19.

U. D. Nielsen. Response-based estimation of sea state parameters - influence of filtering. Ocean Engineering, 34(13):1797 - 1810, 2007. ISSN 0029-8018. doi: 10.1016/j.oceaneng.2007.03.002. Cited on page 3 .

U. D. Nielsen. The wave buoy analogy - estimating high-frequency wave excitations. Applied Ocean Research, 30(2):100-106, 2008. Cited on page 3.

U. D. Nielsen and T. Iseki. The wave buoy analogy: Analysis of synthetic data by bayesian modelling. ASME Conference Proceedings, 2010(49125):301-310, 2010. doi: 10.1115/OMAE2010-20530. Cited on page 3.

U. D. Nielsen and T. Iseki. A study on parametric wave estimation based on measured ship motions. Nihon Kokai Gakkai Ronbunshu, 2011. ISSN 03887405. Cited on page 3.

T. Perez. Ship Motion Control: Course Keeping and Roll Stabilisation Using Rudder and Fins. Advances in Industrial Control Series. Springer-Verlag London Limited, 2005. ISBN 9781846281570. Cited on pages 3, 19, 23, 26, 27, 29, 30, 31,32 , and 116 .

R. Pintelon and J. Schoukens. System Identification: A Frequency Domain Approach. Wiley, 2012. ISBN 9781118287392. Cited on page 7. 
M. Reineh, M. Enqvist, and F. Gustafsson. Detection of roof load for automotive safety systems. In Decision and Control (CDC), 2013 IEEE 52nd Annual Conference on, pages 2840-2845, Dec 2013. doi: 10.1109/CDC.2013.6760314. Cited on page 113 .

A. Ross. Nonlinear Manoeuvring Models for Ships: A Lagrangian Approach. PhD thesis, Norwegian University of Science and Technology, Trondheim, Norway, 2008. Cited on page 26.

J. Schoukens and R. Pintelon. Identification of Linear Systems: A Practical Guideline to Accurate Modeling. Pergamon Press, Inc., Elmsford, NY, USA, 1991. ISBN 0-08-040734-X. Cited on page 7.

S. Skogestad and I. Postlethwaite. Multivariable feedback control: analysis and design. John Wiley, 2005. ISBN 9780470011676. Cited on page 1.

T. Söderström and P. Stoica. Instrumental Variable Methods for System Identification. Lecture Notes in Control and Information Sciences. Springer-Verlag, 1983. ISBN 9783540128144. Cited on page 13.

T. Söderström. Identification of stochastic linear systems in presence of input noise. Automatica, 17(5):713 - 725, 1981. ISSN 0005-1098. doi: 10.1016/0005-1098(81) 90018-2. Cited on page 44.

T. Söderström and P. Stoica. System Identification. Prentice Hall International Series In Systems And Control Engineering. Prentice Hall, 1989. ISBN 9780138812362. Cited on pages 7, 13, 47, and 56.

M. Spong and S. Hutchinson. Robot Modeling and Control. Wiley, 2005. ISBN 9780471649908. Cited on pages 20 and 23.

E. Tannuri, J. Sparano, A. Simos, and J. Da Cruz. Estimating directional wave spectrum based on stationary ship motion measurements. Applied Ocean Research, 25(5):243-261, 2003. Cited on page 3.

S. Thornton and J. Marion. Classical Dynamics of Particles and Systems. Brooks/Cole, Cengage Learning, 2004. ISBN 9780534408961. Cited on pages 20 and 23 .

M. Thys. Theoretical and experimental investigation of a free running fishing vessel at small frequency of encounter. PhD thesis, Norwegian University of Science and Technology, Trondheim, Norway, 2013. Cited on page 93.

C. Tzeng, G. Goodwin, and S. Crisafulli. Feedback linearization design of a ship steering autopilot with saturating and slew rate limiting actuator. International Journal of Adaptive Control and Signal Processing, 13(1):23-30, 1999. Cited on page 8 .

É. Walter. Identifiability of State Space Models: With Applications to Transformation Systems. Lecture Notes in Biomathematics. Springer-Verlag Berlin, 1982. ISBN 9783540115908. Cited on page 16. 


\section{Licentiate Theses \\ Division of Automatic Control \\ Linköping University}

P. Andersson: Adaptive Forgetting through Multiple Models and Adaptive Control of Car Dynamics. Thesis No. 15, 1983.

B. Wahlberg: On Model Simplification in System Identification. Thesis No. 47, 1985.

A. Isaksson: Identification of Time Varying Systems and Applications of System Identification to Signal Processing. Thesis No. 75, 1986.

G. Malmberg: A Study of Adaptive Control Missiles. Thesis No. 76, 1986.

S. Gunnarsson: On the Mean Square Error of Transfer Function Estimates with Applications to Control. Thesis No. 90, 1986.

M. Viberg: On the Adaptive Array Problem. Thesis No. 117, 1987.

K. Ståhl: On the Frequency Domain Analysis of Nonlinear Systems. Thesis No. 137, 1988.

A. Skeppstedt: Construction of Composite Models from Large Data-Sets. Thesis No. 149, 1988.

P. A. J. Nagy: MaMiS: A Programming Environment for Numeric/Symbolic Data Processing. Thesis No. 153, 1988.

K. Forsman: Applications of Constructive Algebra to Control Problems. Thesis No. 231, 1990.

I. Klein: Planning for a Class of Sequential Control Problems. Thesis No. 234, 1990.

F. Gustafsson: Optimal Segmentation of Linear Regression Parameters. Thesis No. 246, 1990.

H. Hjalmarsson: On Estimation of Model Quality in System Identification. Thesis No. 251, 1990.

S. Andersson: Sensor Array Processing; Application to Mobile Communication Systems and Dimension Reduction. Thesis No. 255, 1990.

K. Wang Chen: Observability and Invertibility of Nonlinear Systems: A Differential Algebraic Approach. Thesis No. 282, 1991.

J. Sjöberg: Regularization Issues in Neural Network Models of Dynamical Systems. Thesis No. 366, 1993.

P. Pucar: Segmentation of Laser Range Radar Images Using Hidden Markov Field Models. Thesis No. 403, 1993.

H. Fortell: Volterra and Algebraic Approaches to the Zero Dynamics. Thesis No. 438, 1994.

T. McKelvey: On State-Space Models in System Identification. Thesis No. 447, 1994.

T. Andersson: Concepts and Algorithms for Non-Linear System Identifiability. Thesis No. 448, 1994.

P. Lindskog: Algorithms and Tools for System Identification Using Prior Knowledge. Thesis No. 456, 1994.

J. Plantin: Algebraic Methods for Verification and Control of Discrete Event Dynamic Systems. Thesis No. 501, 1995.

J. Gunnarsson: On Modeling of Discrete Event Dynamic Systems, Using Symbolic Algebraic Methods. Thesis No. 502, 1995.

A. Ericsson: Fast Power Control to Counteract Rayleigh Fading in Cellular Radio Systems. Thesis No. 527, 1995.

M. Jirstrand: Algebraic Methods for Modeling and Design in Control. Thesis No. 540, 1996.

K. Edström: Simulation of Mode Switching Systems Using Switched Bond Graphs. Thesis No. 586, 1996. 
J. Palmqvist: On Integrity Monitoring of Integrated Navigation Systems. Thesis No. 600, 1997.

A. Stenman: Just-in-Time Models with Applications to Dynamical Systems. Thesis No. 601, 1997.

M. Andersson: Experimental Design and Updating of Finite Element Models. Thesis No. 611, 1997.

U. Forssell: Properties and Usage of Closed-Loop Identification Methods. Thesis No. 641, 1997.

M. Larsson: On Modeling and Diagnosis of Discrete Event Dynamic systems. Thesis No. 648, 1997.

N. Bergman: Bayesian Inference in Terrain Navigation. Thesis No. 649, 1997.

V. Einarsson: On Verification of Switched Systems Using Abstractions. Thesis No. 705, 1998.

J. Blom, F. Gunnarsson: Power Control in Cellular Radio Systems. Thesis No. 706, 1998.

P. Spångéus: Hybrid Control using LP and LMI methods - Some Applications. Thesis No. 724, 1998.

M. Norrlöf: On Analysis and Implementation of Iterative Learning Control. Thesis No. 727, 1998.

A. Hagenblad: Aspects of the Identification of Wiener Models. Thesis No. 793, 1999.

F. Tjärnström: Quality Estimation of Approximate Models. Thesis No. 810, 2000.

C. Carlsson: Vehicle Size and Orientation Estimation Using Geometric Fitting. Thesis No. 840, 2000.

J. Löfberg: Linear Model Predictive Control: Stability and Robustness. Thesis No. 866, 2001.

O. Härkegård: Flight Control Design Using Backstepping. Thesis No. 875, 2001.

J. Elbornsson: Equalization of Distortion in A/D Converters. Thesis No. 883, 2001.

J. Roll: Robust Verification and Identification of Piecewise Affine Systems. Thesis No. 899, 2001.

I. Lind: Regressor Selection in System Identification using ANOVA. Thesis No. 921, 2001.

R. Karlsson: Simulation Based Methods for Target Tracking. Thesis No. 930, 2002.

P.-J. Nordlund: Sequential Monte Carlo Filters and Integrated Navigation. Thesis No. 945, 2002.

M. Östring: Identification, Diagnosis, and Control of a Flexible Robot Arm. Thesis No. 948, 2002.

C. Olsson: Active Engine Vibration Isolation using Feedback Control. Thesis No. 968, 2002 .

J. Jansson: Tracking and Decision Making for Automotive Collision Avoidance. Thesis No. 965, 2002.

N. Persson: Event Based Sampling with Application to Spectral Estimation. Thesis No. 981, 2002.

D. Lindgren: Subspace Selection Techniques for Classification Problems. Thesis No. 995, 2002.

E. Geijer Lundin: Uplink Load in CDMA Cellular Systems. Thesis No. 1045, 2003.

M. Enqvist: Some Results on Linear Models of Nonlinear Systems. Thesis No. 1046, 2003.

T. Schön: On Computational Methods for Nonlinear Estimation. Thesis No. 1047, 2003.

F. Gunnarsson: On Modeling and Control of Network Queue Dynamics. Thesis No. 1048, 2003.

S. Björklund: A Survey and Comparison of Time-Delay Estimation Methods in Linear Systems. Thesis No. 1061, 2003. 
M. Gerdin: Parameter Estimation in Linear Descriptor Systems. Thesis No. 1085, 2004.

A. Eidehall: An Automotive Lane Guidance System. Thesis No. 1122, 2004.

E. Wernholt: On Multivariable and Nonlinear Identification of Industrial Robots. Thesis No. 1131, 2004.

J. Gillberg: Methods for Frequency Domain Estimation of Continuous-Time Models. Thesis No. 1133, 2004.

G. Hendeby: Fundamental Estimation and Detection Limits in Linear Non-Gaussian Systems. Thesis No. 1199, 2005.

D. Axehill: Applications of Integer Quadratic Programming in Control and Communication. Thesis No. 1218, 2005.

J. Sjöberg: Some Results On Optimal Control for Nonlinear Descriptor Systems. Thesis No. 1227, 2006.

D. Törnqvist: Statistical Fault Detection with Applications to IMU Disturbances. Thesis No. 1258, 2006.

H. Tidefelt: Structural algorithms and perturbations in differential-algebraic equations. Thesis No. 1318, 2007.

S. Moberg: On Modeling and Control of Flexible Manipulators. Thesis No. 1336, 2007.

J. Wallén: On Kinematic Modelling and Iterative Learning Control of Industrial Robots. Thesis No. 1343, 2008.

J. Harju Johansson: A Structure Utilizing Inexact Primal-Dual Interior-Point Method for Analysis of Linear Differential Inclusions. Thesis No. 1367, 2008.

J. D. Hol: Pose Estimation and Calibration Algorithms for Vision and Inertial Sensors. Thesis No. 1370, 2008.

H. Ohlsson: Regression on Manifolds with Implications for System Identification. Thesis No. 1382, 2008.

D. Ankelhed: On low order controller synthesis using rational constraints. Thesis No. 1398, 2009.

P. Skoglar: Planning Methods for Aerial Exploration and Ground Target Tracking. Thesis No. 1420, 2009.

C. Lundquist: Automotive Sensor Fusion for Situation Awareness. Thesis No. 1422, 2009.

C. Lyzell: Initialization Methods for System Identification. Thesis No. 1426, 2009.

R. Falkeborn: Structure exploitation in semidefinite programming for control. Thesis No. 1430, 2010.

D. Petersson: Nonlinear Optimization Approaches to $\mathcal{H}_{2}$-Norm Based LPV Modelling and Control. Thesis No. 1453, 2010.

Z. Sjanic: Navigation and SAR Auto-focusing in a Sensor Fusion Framework. Thesis No. 1464, 2011.

K. Granström: Loop detection and extended target tracking using laser data. Thesis No. 1465, 2011.

J. Callmer: Topics in Localization and Mapping. Thesis No. 1489, 2011.

F. Lindsten: Rao-Blackwellised particle methods for inference and identification. Thesis No. 1480, 2011.

M. Skoglund: Visual Inertial Navigation and Calibration. Thesis No. 1500, 2011.

S. Khoshfetrat Pakazad: Topics in Robustness Analysis. Thesis No. 1512, 2011.

P. Axelsson: On Sensor Fusion Applied to Industrial Manipulators. Thesis No. 1511, 2011.

A. Carvalho Bittencourt: On Modeling and Diagnosis of Friction and Wear in Industrial Robots. Thesis No. 1516, 2012. 
P. Rosander: Averaging level control in the presence of frequent inlet flow upsets. Thesis No. 1527, 2012.

N. Wahlström: Localization using Magnetometers and Light Sensors. Thesis No. 1581, 2013.

R. Larsson: System Identification of Flight Mechanical Characteristics. Thesis No. 1599, 2013.

Y. Jung: Estimation of Inverse Models Applied to Power Amplifier Predistortion. Thesis No. 1605, 2013.

M. Syldatk: On Calibration of Ground Sensor Networks. Thesis No. 1611, 2013.

M. Roth: Kalman Filters for Nonlinear Systems and Heavy-Tailed Noise. Thesis No. 1613, 2013.

D. Simon: Model Predictive Control in Flight Control Design — Stability and Reference Tracking. Thesis No. 1642, 2014.

J. Dahlin: Sequential Monte Carlo for inference in nonlinear state space models. Thesis No. 1652, 2014.

M. Kok: Probabilistic modeling for positioning applications using inertial sensors. Thesis No. 1656, 2014. 